\title{
AMNIOTE PHYLOGENY AND THE IMPORTANCE OF FOSSILS
}

\author{
Jacques Gauthier ${ }^{1,3}$, Arnold G. Kluge', \\ and Timothy Rowe ${ }^{2}$ \\ ${ }^{1}$ Museum of Zoology and Department of Biology, University of Michigan, \\ Ann Arbor, MI 48109-1079; \\ ${ }^{2}$ Department of Geological Sciences, University of Texas, \\ Austin, TX 78713-7909, U.S.A.
}

\begin{abstract}
Several prominent cladists have questioned the importance of fossils in phylogenctic inference, and it is becoming increasingly popular to simply fit extinct forms, if they are considered at all, to a cladogram of Recent taxa. Gardiner's (1982) and Lovtrup's (1985) study of amniote phylogeny exemplifies this differential treatment, and we focused on that group of organisms to test the proposition that fossils cannot overturn a theory of relationships based only on the Recent biota. Our parsimony analysis of amniote phylogeny, special knowledge contributed by fossils being scrupulously avoided, led to the following best fitting classification, which is similar to the novel hypothesis Gardiner published: (lepidosaurs (turtles (mammals (birds, crocodiles)))). Howcver, adding fossils resulted in a markedly different most parsimonious cladogram of the extant taxa: (mammals (turtles (lepidosaurs (birds, crocodiles )))). That classification is like the traditional hypothesis, and it provides a better fit to the stratigraphic record. 'To isolate the extinct taxa responsible for the latter classification, the data were successively partitioned with cach phylogenetic analysis, and we concluded that: (1) the ingroup, not the outgroup, fossils were important; (2) synapsid, not reptile, fossils were pivotal; (3) certain synapsid fossils, not the earliest or latest, were responsible. The critical nature of the synapsid fossils seemed to lie in the particular combination of primitive and derived character states they exhibited. Classifying those fossils, along with mammals, as the sister group to the lineage consisting of birds and crocodiles resulted in a relatively poor fit to data; one involving a 2-4 fold increase in cvolutionary reversals! Thus, the importance of the critical fossils, collectively or individually, seems to reside in their relative primitiveness, and the simplest explanation for their more conservative nature is that they have had less time to evolve. While fossils may be important in phylogenetic inference only under certain conditions, there is no compelling reason to prejudge their contribution. We urge systematists to evaluate fairly all of the available evidence.
\end{abstract}

\begin{abstract}
"Some day, no doubt, when the exact relationship of the various living and extinct reptiles has been more accurately determined, it will be necessary to split up the artificial group Reptilia, assigning some to the Mammalia and some to the Aves..."
\end{abstract}

Edwin S. Goodrich (1916: 261)

\section{Introduction}

Systcmatists have claimed for many years that mammals are related to other extant amniotes only through a long line of extinct forms. Moreover, they have argued that crocodiles and birds are one another's nearest living relatives, and that lepidosaurs are the sister lineage to that group. Currently, the two principal problems in the phylogeny of extant amniotes are believed to be the relationships of turtles and mammals. In this context, Gardiner's (1982) analysis of tetrapod phylogeny is remarkable, because he delimited groups of extant amniotes that had previously been recognized by some comparative anatomists operating under pre-Darwinian paradigms. Perhaps most surprising was the group (birds, mammals), which he referred to as Haemothermia. This name was erected for the same assemblage by the staunch anti-evolutionist - or, to be

Present Address: Department of Herpetology, California Academy of Sciences, San Francisco, CA 94118 9961, U.S.A. 
generous, anti-Darwinist (Gould, 1986)-Sir Richard Owen (1866). Gardiner also considered crocodiles to be the sister group to Haemothermia, and he referred all three taxa to Owen's Thecodontia. Furthermore, Gardiner concluded that turtles and Thecodontia are sister groups, which constitute Euamniota, and that lepidosaurs (squamates, Sphenodon) are the sister group to all other Recent amniotes. Gardiner's arrangement of amniotes (Fig. 1) is at odds with fossil-based hypotheses that paleontologists have presented since the time of Darwin. Except for the placement of Sphenodon, Løvtrup (1985) claimed to have found additional support for Gardiner's phylogeny of extant amniotes. Such corroboration suggests that this phylogenetic hypothesis deserves to be taken seriously and critically re-examined.

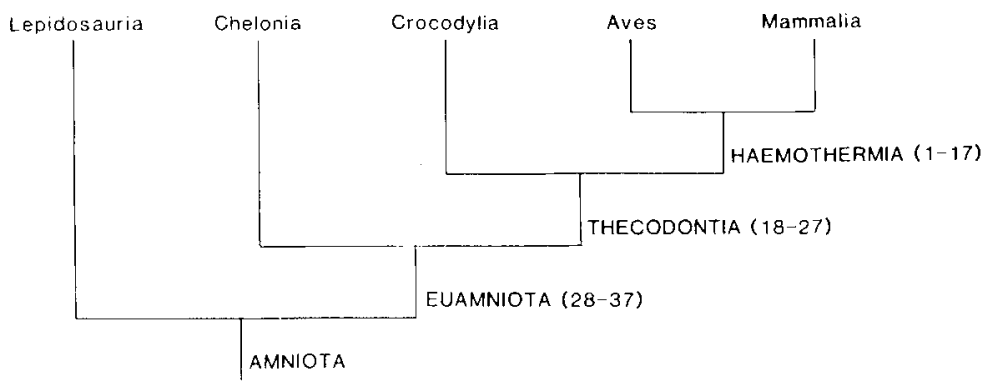

Fig. 1. The sister group relationships among the five assemblages of living amniotes advocated by Gardiner (1982; see also Lovtrup, 1985). The numbers of the synapomorphies Gardiner employed are displayed on the. cladogram. Redrawn from Gardiner (1982: fig. 2).

Most of Gardiner's (1982) evidence for the relationships among amniotes could only be observed on extant forms. He later fitted some of the better known fossil taxa within this framework based on observations of both extinct and extant organisms. This practice has been advocated by other prominent cladists and it is becoming increasingly popular. Justification for such unequal treatment of extinct and extant taxa has been presented by Crowson (1970), Hennig (1981; see also 1966), Lovtrup (1977), Patterson (1977a; 1981a,b; 1982) and Rosen et al. (1981: 178).

We agree with many of the criticisms leveled at what has been called the paleontological method. At its worst, it reduces to a preoccupation with discovering ancestors, uses overall similarity as evidence of relationships, and equates stratigraphic position with direction of evolutionary change. However, Gardiner's radical hypothesis of amniote phylogeny caused us to look more closely at one of his central propositions, which was, as Patterson put it (1981a: 218; see also 1981b), "instances of fossils overturning theories of relationship based on Recent organisms are very rare, and may be nonexistent." At least superficially, this seems an eminently reasonable proposition. After all, the fossil record is incomplete, and even under the best of conditions the fossils themselves are but parts of individual organisms. Much larger samples of extant species can usually be obtained, and the anatomy and histology of soft tissues, as well as embryology, physiology, molecules, chromosomes, and ethology, are available for study.

Amniote phylogeny seems a perfect test of the proposition that fossils are so uninformative that they can have little or no impact on a hypothesis derived from the comparatively information-rich extant biota. The major groups of extant amniotes, mammals, turtles, lepidosaurs, crocodiles and birds, are well known and quite different 
from one another, and each of these groups has a long and reasonably well-known fossil record. Moreover, knowledge of fossils is thought to have played a central role in the development of ideas concerning amniote phylogeny. If fossils are of little aid in unravelling the genealogy of such markedly different organisms as turtles and birds, then indeed one would be on tenuous ground when asserting that fossils are, in general, important in phylogenetic inference.

\section{Data and Methods of Analysis}

Gardiner (1982, fig. 2) used 37 synapomorphies to resolve the phylogenetic relationships among the five major groups of extant amniotes (Fig. 1). Hereafter, all references to Gardiner, without more specific citation, can be assumed to be to his 1982 paper. It is readily apparent to many comparative biologists that at least some of Gardiner's characters, as well as Løvtrup's (1985), were incorrectly interpreted. We have identified five classes of mistakes made by these authors. They misinterpreted either (1) the character descriptions in the literature (e.g., see characters G25 and L16 below), or (2) the taxonomic distribution of a character (e.g., G31 and L12); (3) they listed redundant characters (e.g., G1); (4) matching character states failed the pretesting criteria for homology (Patterson, 1982; Rieppel, 1980; Stevens, 1984); and (5) the direction of character state evolution was misjudged (de Queiroz, 1985; Kluge, 1985; Kluge and Strauss, 1985; Maddison et al., 1984). Thus, we have reanalyzed Gardiner's and Lovtrup's evidence to blunt the criticism that we have "set up a strawman".

Gardiner's characters are discussed separately under the heading "Gardiner's Data". Each character is labelled with a $G$ throughout the text, Table 1, and Appendix A, and his numbering system (as in Fig. 1) is retained for ease of comparing his conclusions with ours. We added a lower case letter to those characters that were substantially redefined (e.g., G4a). Gardiner used seven characters to diagnose Amniota (38-44, Fig. 1). We see no reason to doubt the verity of that taxon (Gauthier et al., 1988b); however, we reanalyzed some of those characters, because they appeared to diagnose clades within Amniota. Our conclusions are summarized in Table 1.

The additional evidence for amniote relationships cited by Løvtrup (1985) was also re-evaluated (see section "Løvtrup's Data" and Table 1). Unless stated otherwise, all further references to Løvtrup denote his 1985 paper. Most of the characters summarized by him (including those attributed to Gardiner) were presented in three tables. His table 1 was a diagnosis of Haemothermia, table 2 of Thecodontia (sensu Gardiner) and table 3 of Testudines + Thecodontia (Gardiner's Euamniota). Given the redundant character numbers in these separate tables, we renumberd his 16 characters (e.g., Lla, L4, ... in Table 1). Each of Løvtrup's characters is cross-referenced in our text according to the table and character number he used (e.g., table 2:11, the "Absence or reduction of Jacobson's organ," is L6a).

Additional, potentially useful, information on amniote relationships was encountered during our review of Gardiner's and Løvtrup's data. In fact, Gardiner's own paper was a major resource; he discussed nearly twice the number of characters summarized in his figure 2 (Fig. 1). We have considered some of this additional evidence, for reasons of efficiency, in the context of our discussions of Gardiner's and Løvtrup's "preferred" synapomorphies. These additional characters are also denoted with a lower case letter in Table 1 (e.g., G12a, or Lla). Some of our discoveries bear little or no structural or functional similarity to the characters Gardiner and Lovtrup emphasized, and those 
variables are discussed separately under the heading "Additional Data" (A1, A2, A3 ... in Table 1 ).

Many of the additional characters we found are from the "hard", or fossilizable anatomy, and in most cases they can be described succinctly as the presence or absence of a bone (e.g., A30-53). As with the "soft", or less preservable anatomy, these data were scored and polarized entirely on the basis of their distribution among Recent choanates. We reanalyzed Recent amniote relationships in terms of soft, hard, and soft + hard characters. The following characters in Table 1 are considered the hard data: G9, G1 1, G1 la, G11b, G18, G19, G19a, G23, G26-27, G36a, L17, A21-24, A27-28, A30-53. The remainder are referred to as the soft subset. Several reasons for the paucity of evidence from the skeleton of Recent amniotes are considered in the Discussion.

In our reanalysis of Gardiner's and Løvtrup's characters, synapomorphies that failed the pretesting criteria of similarity and conjunction of Patterson (1982; see also Rieppel, 1980; Stevens, 1984) were not considered good estimators of homology. Failing synapomorphies that could not be restated in a manner consistent with the pretesting criteria were discarded. What constitutes a sufficient degree of similarity was complicated by the different aspects that may be assessed in terms of composition, development, and topography. For example, Gardiner suggested that the side wall of the braincase is homologous in birds, crocodiles and mammals, i.e., the laterosphenoid (pleurosphenoid) is homologous with the alisphenoid and epipterygoid (G19). It is true that these bones are similar in that they enclose the brain. However, they develop from what are universally regarded as non-homologous elements and maintain substantially different adult anatomical relations. The laterosphenoid (pleurosphenoid) develops from the pila antotica, a neurocranial element, whereas the epipterygoid-alisphenoid develops at least in part from the palatoquadrate, a visceral arch element. The pila antotica and laterosphenoid lie medial to the cavum epiptericum in adults, but the palatoquadrate and epipterygoid-alisphenoid lic lateral to the cavum. Thus, we recognized the birdcrocodile condition as a synapomorphy in Table 1 , but excluded the mammal state. In the case of the character "fenestra rotunda" (A21), we accepted a modified interpretation of the character, transformations leading to the subdivision of the metotic fissure may fail the test of topography, but the end result is that part of the embryonic fissure remains open and is covered by a secondary tympanic membrane. Our decisions in the reanalysis of Gardiner's and Løvtrup's characters raise thorny conceptual and operational issues, and we are not sure that all our actions can be defended. Nevertheless, we did consider shared apomorphies arising at any point in ontogeny as being potential homologues for the ingroup taxa possessing them, and we did so regardless of dissimilarities in other details of that transformation $(=$ character sensu de Queiroz, 1985; Kluge, 1988). We also recognize that a character may fail a pretesting criterion and still be homologous. In these instances, we are content to let our colleagues and congruence inform us of such mistakes. The alternative seems to invite phylogenetic analyses derived from virtually boundless claims of "similarity".

Those familiar with the tetrapod skeleton will see, even with a casual glance, that many of the hard characters in Table 1 are probably incorrectly interpreted. For example, postorbital bones have been lost in both extant dipnoans and lissamphibians (A35). However, the conclusion of "loss" can only be reached in the context of their fossil relatives, and we were constrained to only extant dipnoans and lissamphibians for information in developing Table 1. Thus, although we are well aware that we have it backwards, we are nonetheless forced to consider the postorbital bone as a neomorph 
that appeared within amniotes. We have no other alternative if we are to measure fairly the robustness of a phylogeny derived exclusively from knowledge of Recent taxa (Table 1).

There are also some hard characters listed in Table 1 that are absent from the fossil data matrix (Table 2). For example, the hypapophyses on the anterior trunk and posterior cervical vertebrae of Recent crocodiles and birds are considered a derived character (A22), because they are absent elsewhere in extant choanates. This character does not, however, appear in the list of "Fossil Data", because our knowledge of the fossil record indicates that these hypapophyses are not homologous. That is to say, we have abundant evidence that hypapophyses arose both within crocodiles (Eusuchia; Romer, 1956) and within the line of coelurosaurs of which birds are a part (Maniraptora; Gauthier, 1986), and not in the common archosaurian ancestor of crocodiles and birds. These hard characters are not included in Appendices $A$ and $B$.

We emphasize that many of the hard characters in Table 1 are questionable only in the context of the more complete knowledge afforded by considering both extinct and extant taxa. This casts a different light upon our information on soft anatomy (Table 1), because in this case our analyses must be confined almost exclusively to extant taxa. $\Lambda \mathrm{s}$ D. B. Wake (pers. comm.) asked, how far off the mark might our conclusions regarding the origin of soft anatomical characters among extant tetrapods seem if we also had knowledge of these data in extinct taxa?

Contrary to Løvtrup's claim, we believe there exists overwhelming evidence for the monophyly of Lepidosauria (squamates + Sphenodon; see diagnosis in Gauthier et al., 1988a). Aside from Lovtrup (see also 1977), no one in the 20th century has accepted the thesis that Sphenodon is the sister group of turtles, crocodiles, birds and mammals. Løvtrup argued that such a placement for Sphenodon, the only living rhynchocephalian, is in keeping with the "classical view", and cited Benton (1984) to that effect (p. 463). It appears that Løvtrup confused rhynchocephalian lepidosauromorphs with rhynchosaurian archosauromorphs, because these taxa had previously been considered members of a paraphyletic "Rhynchocephalia" (Gauthier et al., 1988a). The six characters Løvtrup (p. 466) used to support his placement of Sphenodon are problematic. We have retained his "uncinate processes on ribs" ( $\mathrm{L} 17$ ), but disagree as to the level of generality. His second character, "solid ovaries", was used by Gardiner (G31) to diagnose his Euamniota; we discuss it in that context. "Lime-impregnated shell" is considered in the context of Løvtrup's "similarities in egg shell" (table 3:15; L15). His "albumen" and "horny caruncle" characters are L18 and LI9, respectively, in our reanalysis. The last character, "pars tuberalis", was presented as part of his Haemothermia diagnosis (table $1: 19)$, and we review it in that context (L2).

We assume the existence of two outgroup nodes (Maddison et al., 1984), Tetrapoda and Choanata, in polarizing the characters in Table 1. We accept Gardiner's conclusion regarding the monophyly of Gymnophiona, Anura, and Urodela (p. 219), and we use the name Lissamphibia for that assemblage. Principally because caecilians are so modified, phylogenetic relationships among lissamphibian lineages remain equivocal, and we treat them as an unresolved polytomy in our reanalysis. Lissamphibia + Amniota comprises Tetrapoda in this paper. We employ Recent dipnoans as our necessary second outgroup, following the conclusions of Rosen et al. (1981). Thus, Dipnoi + 'Tetrapoda is equivalent to our Choanata. Schultze's (1986) classification, in which the living Latimeria is the sister group to Tetrapoda, does not appear to be well supported (Forey, 1986). Given the higher classification of choanates that we accept and 
the ancestral state among amniotes, the condition of the Ancestor in Table 1 is characteristic of Tetrapoda ancestrally.

The following 29 groups of amniotes were used to assess the importance of fossils: Araeoscelidia +, Biarmosuchia +, Captorhinidae +, Casea + (represents a group composed of caseids and varanopsids), Choristodera,+ Diademodon + , Dicynodontia + , Dinocephalia +, Edaphosaurus +, Erythrosuchidae+, Exaeretodon +, Gorgonopsia +, Lepidosauromorpha, Mammalia, Morganucodontidae+, Ophiacodon + , Ornithosuchia, Procynosuchus, Proterochampsidae +, Proterosuchidae + , Protorosauria + , Pseudosuchia, Rhynchosauria + , Sphenacodontinae + , Testudines, Therocephalia + , Thrinaxodon + , Trilophosaurus + , Tritylodontidae + . We use these taxa because they are reasonably well known and have been interpreted previously as synapsids or reptiles (Fig. 3). Each taxon marked with a cross $(+)$ is an extinct lineage; the remainder include both extant and extinct species. Lepidosauromorpha, Ornithosuchia and Pseudosuchia include living squamates and Sphenodon, birds, and crocodilians, respectively. Evidence used to analyze relationships among these 29 taxa is briefly described under the heading "Fossil Data", and the taxonomic distribution and polarity of the characters are summarized in Table 2. Limitations on space have forced us to keep these character descriptions brief. Further documentation can be found in Gauthier (1984) and Rowe (1986a), whose hypotheses were developed largely from comparative anatomical evidence already in the literature, and were thus available to both Gardiner and Løvtrup. The ancestral state for each of these characters was deduced from the conditions present in a composite first outgroup including non-amniote batrachosaurs, such as Diadectes, Kotlassia, Limnoscelis, Seymouria, and Tseajaia (Heaton, 1980), together with a second composite outgroup including non-batrachosaur tetrapods, such as Anthracosaurus, Archeria, Eogyrinus, Gephyrostegus, and Palaeoherpeton (Panchen, 1980).

For Recent terminal taxa, within- and between-taxon variation in the soft and hard characters are discussed in the text, but only the latter variation is noted in Table 1 . In order to reduce the size of our manuscript, both classes of variation are summarized for those characters listed in Table 2. Variants within the terminal taxa are separated by slashes in Table 2, and the state to the left is considered diagnostic of the taxon.

Computer programs (e.g., PAUP; see below) used in phylogenetic analysis of additively coded binary data accept data matrices consisting of only one state per character per taxon. Thus, variable terminal taxa, like we have observed, are a special problem. Subdividing each such entry into invariant taxonomic components provides a solution; however, that strategy becomes increasingly impractical the greater the variation. Our data matrices contain several terminal taxa that are variable in more than one character, and we did not attempt to solve the problem by taxonomic subdivision. Another option was available, however, in those cases where the character states could be unambiguously optimized (see below) on a well-documented hypothesis of relationship among the members of the variable terminal taxa, as described in Gauthier (1984) and Rowe (1986a). In those relatively few situations where the optimization was equivocal or an ingroup hypothesis was lacking, we chose the zero state as ancestral for the variable terminal taxon. Using the apomorphy is probably a more biased choice, because when that state is shared it definitely provides evidence of relationship of the variable terminal taxon.

All multistate characters were treated as unordered to avoid biasing our conclusions by the traditional, theory-laden interpretations of how such transformation series are supposed to have evolved. Some character states were indeterminable. Occasionally, a 
character did not apply to a taxon (e.g., the presence or absence of pores in the egg shell of amniotes cannot be interpreted in the outgroups, which lack egg shells), and it is entered in Table l (L15a) as an N, signifying "not applicable". Where data were simply unavailable, as was often the case with fossils, the missing observations are represented by a?. For simplicity, autapomorphies have been omitted from Tables 1 and 2. Swofford's (1984) mainframe computer software PAUP, Phylogenetic Analysis Using Parsimony, was used to analyze these data. The data in Table 1 were analyzed using the branchand-bound algorithm (Hendy and Penny, 1982), while those in Table 2 were treated with the global branch-swapping and multiple parsimony tree options. A single shortest length tree was found (Figs 2 and 3 ).

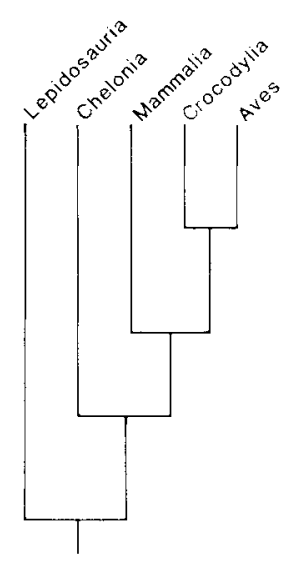

Fig. 2. The single cladogram that best fits the data from the five major groups of living amniotes summarized in ' lable 1. The cladogram is 175 steps long and the consistency index is 0.674 . We refer to this hypothesis as the Recent tree. 'Three of the four groups are the same as Gardiner (1982) delimited (see Fig. 1 ).

The tree in Fig. 2 is referred to as the Recent hypothesis, because it is based only on extant taxa. It is similar to Gardiner's proposition (Fig. 1), three of the four groups being the same. While many of the observations used to produce Fig. 3 (Tables 1, in part, and 2) were taken from the work of others, we take responsibility for the sampling, character interpretations, and resulting phylogenetic hypothesis. Accordingly, we refer to that tree as ours.

We optimize, according to the rule of parsimony, each of Gardiner's and Løvtrup's characters on these alternative phylogenetic hypotheses in order to identify the diagnostic value of the character states. To e-mphasize that these are a posteriori conditions, which were made subsequent to our reanalysis of aminiote phylogeny (Figs 2,3), we place these discussions in parentheses, immediately following the descriptions of the characters involved. The optimal, most parsimonous, character state distribution in the context of a particular cladogram is ambiguous (equivocal) when the point of origin can be attributed to two or more adjacent nodes, otherwise it is unambiguous (unequivocal). Ambiguous characters lend themselves to several equally parsimonious interpretations as to their diagnostic information; these possibilities are summarized in Appendix A. An unambiguous character should not, however, be taken to mean that it is unique and unreversed. On the contrary, a given character can have a complex history of origins and reversals, and nonetheless be unambiguous if its distribution on a 


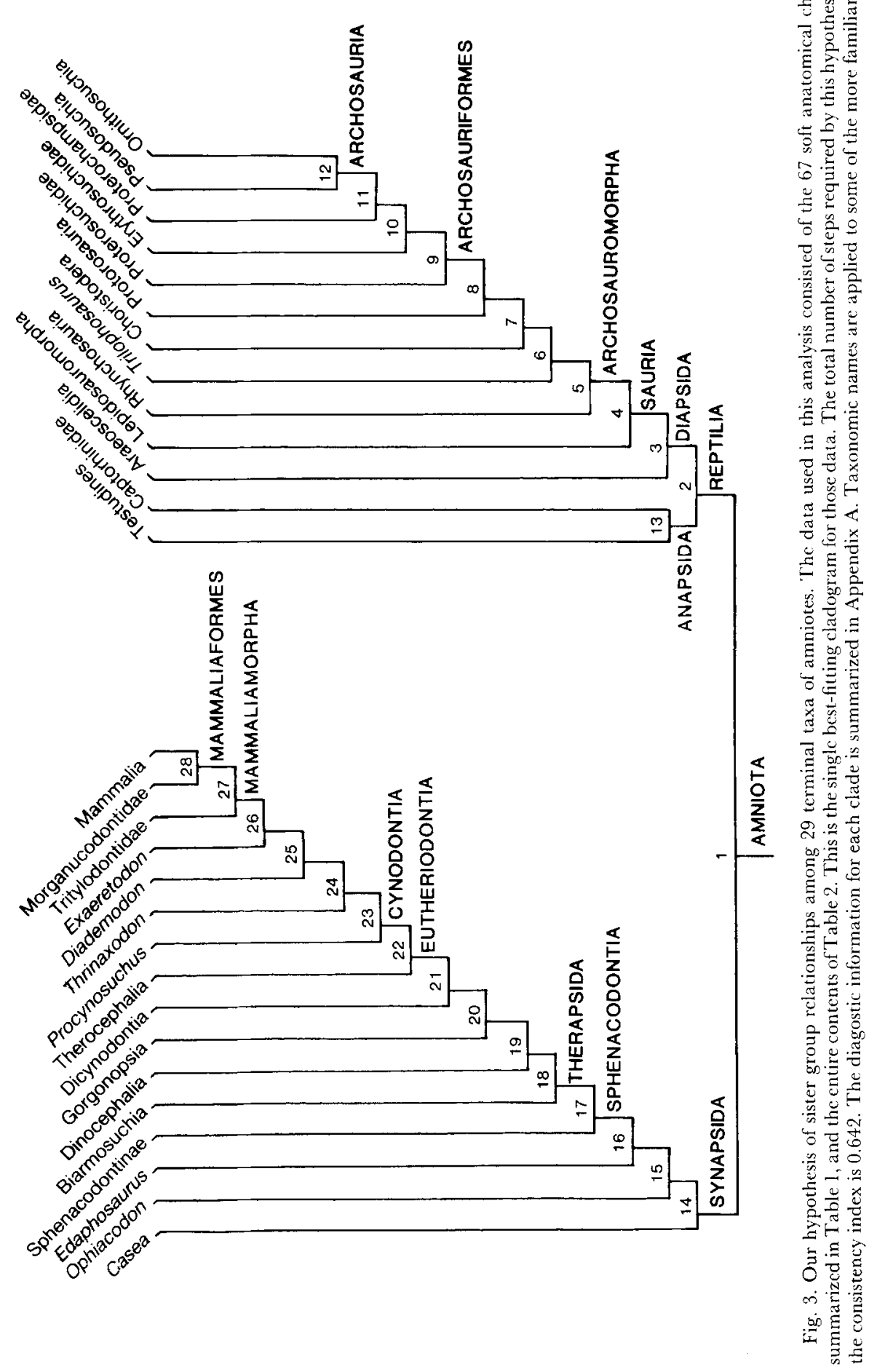


particular cladogram leads us to this conclusion. For example, one must conclude that the position of the craniomandibular joint with respect to the occiput has had a complex history among amniotes (character 70 in Table 2; Fig. 3). An amorphic condition in which the joint lies posterior to the occiput arose in synapsids ancestrally and subsequently reversed in therapsids. This character also arose in archosauromorphs, and has reversed or developed other apomorphic conditions several times within that group. Nevertheless, the hypothesized history of this character is unambiguous on our tree (Fig. 3; see also $\Lambda$ ppendix A). Conversely, although a character displays little variation, its history may still be equivocal on our tree. For example, the sinus venosus $(\mathrm{G} 21)$ is absent (ancestral) in turtles and present (derived) in all other amniotes. Thus, although the presence of a sinus venosus shows little variation, its distribution among amniotes leaves its history as ambiguous, according to our hypothesis, in that it could have appeared in amniotes and reversed in turtles, or it could have evolved independently in mammals and saurians.

\section{Gardiner's Data}

\section{Separation of Oxygenated and Deoxygenated Blood}

Several problems were encountered in our reanalysis of this character. As stated, Gardiner confused form and function; it is widely accepted that analogous modifications can perform the same functions (e.g., the wings of birds, bats, and pterosaurs). Moreover, the separation of oxygenated and deoxygenated blood is a consequence of having a certain set of anatomical traits. Since Gardiner also used some of these conditions as synapomorphies, such as a completely divided heart (see character 20 below), there is an obvious lack of independence in the evidence. Further, since we conclude that the interventricular septa of birds and mammals (see character 20 below) are not homologous, it might be inferred that the oxygenated-deoxygenated synapomorphy uniting birds and mammals is equally unfounded. Taken at face value, the complete separation of the two blood types is also characteristic of the crocodile heart, and therefore more accurately diagnoses Gardiner's Thecodontia. Any mixing that might be associated with the foramen Panizzae in crocodiles is considered irrelevant to this point, because it does not involve heart chambers. The foramen Panizzae allows blood to bypass the pulmonary pathway during sustained diving in crocodiles and a few lineages of turtles (Kennedy and Brockman, 1965). The separation of blood types has not been used in our reanalysis of amniote relationships, because of its apparent redundancy with related morphological novelties that we consider below.

Gardiner mentioned (p. 212) the similar developmental histories of the interauricular septa in birds and monotremes in his general discussion of the heart, implying the existence of additional evidence for Haemothermia. Birds, and presumably monotremes, are like all other non-placental tetrapods in that the interauricular septum develops "from the dorso-anterior area between the two atrial [auricular] lobes... [and]... grows toward the endocardial cushion area and unites with the cushion septum" (Nelsen, 1953: 754). Small openings, fenestrae ovale, occur in the septum and normally close during hatching or birth. The formation of the interauricular septum and the foramen ovale are considerably more complicated in placental mammals (Nelsen, 1953; Lawson, 1979). The development of the bird-monotreme foramen ovale appears to be ancestral and, therefore, it has not been included in our reanalysis. 
Also, we have been unable to confirm Gardiner's proposition (see also Griffiths, 1978) that the great cardiac vein opens directly into the right auricle only in birds, monotremes and marsupials. According to Smith (1960), placental mammals are unique in that a vestige of the left precava, the coronary sinus, transports blood directly to the right auricle; all other tetrapods, including birds, monotremes and marsupials, exhibit the ancestral condition.

\section{Single Aortic Trunk with Three Semilunar Valves}

The nature of the semilunar valves will be discussed under character 3 . That adult birds and mammals both have a single aortic trunk, rather than the paired condition observed in other amniotes, is not disputed. However, whether or not the ventral aorta of birds and mammals has a similar developmental history, involving internal subdivision by the aortic septum (Holmes, 1975a: fig. 12), to produce effective right and left aortae, is an important and debatable question. According to Benninghoff (1933), the avian condition represents a fusion of right and left aortae. A totally different proposition, the one adopted by Gardiner, was put forward by Goodrich (1930: 561-562). Goodrich claimed that birds are like mammals in that the aortic septum never develops. There seems to be no way to resolve the disagreement between Goodrich and Benninghoff short of re-examining the embryology of the bird ventral aorta. Nevertheless, since the more recent works of Holmes (1975a,b) and Huettner (1966: 224) support Gardiner's interpretation, we tentatively accept the conclusion that the adult bird and mammal conditions are the same throughout ontogeny, and we judge this state to be apomorphic. The condition seen in other adult tetrapods, with the single aorta subdivided by a valve which leads into right and left systemic channels, is considered ancestral (Holmes, 1975a: fig. 12).

(In the context of the Recent tree (Fig. 2), this character is equivocal. Either a single aorta arose in Thecodontia and reversed in crocodiles, or it originated independently in birds and mammals. According to our hypothesis (Fig. 3), the single aortic trunk is not homologous.)

\section{Pulmonary Artery with Three Semilunar Valves}

Gardiner maintained that birds and mammals have three semilunar valves at the origin of the aorta (character 2, in part) and the pulmonary artery; two such valves are present in other amniotes (Bhatia, 1929). It seems, however, that crocodiles are identical to birds and mammals (Beddard and Mitchell, 1895: 348), and therefore this apomorphy is shared by the members of Gardiner's more inclusive 'Thecodontia (Table 1: G3).

Gardiner emphasized (p. 212), but did not explicitly designate as a synapomorphy (fig. 2), the nature of the left auriculo-ventricular valve. He noted that it consists of three membranous folds in birds and monotremes, one in other non-mammalian amniotes, and two in placental mammals. We have been unable to confirm these generalizations. According to Beddard and Mitchell (1895), Goodrich (1930), Kashyap (1960), and I awson (1979), the left valve consists of one or two folds in all tetrapods, except monotremes which have three (Griffiths, 1978). The number of folds making up the left auriculo-ventricular valve appears uninformative and therefore we have not used that character.

There may be some phylogenetically useful variation in the fleshiness of the left 
auriculo-ventricular valves, but this variation requires further study. We can add, with some assurance, that crocodiles and birds are alone among amniotes in possessing a muscular lateral valve of the right auriculo-ventricular orifice (Goodrich, 1930). This character is labelled G3a in Table 1.

(According to the Recent tree (Fig. 2), three semilunar valves arose in Thecodontia. Our hypothesis (Fig. 3), however, indicates the valves evolved independently in mammals and archosaurs. The presence of a muscular lateral valve of the right auriculoventricular orifice, diagnoses Archosauria on both trees; Figs 2 and 3.)

\section{ENDOTHERMY}

Gardiner noted (p. 213) that the resting metabolic rate of birds and mammals is at least five times higher than all other amniotes of comparable size and similar body temperature. We tentatively accept this character as a potential bird-mammal synapomorphy (Table 1: G4). Endothermy, however, is a complex function of at least two variables: aerobic capacity and metabolic rate. Considering no other evidence, one could argue that therian mammals, in general, and placentals, in particular, are closer to birds (especially passerines) than are monotremes (see character 5), because they share a similar increased aerobic capacity and metabolic rate.

Gardiner also considered incubating eggs diagnostic of birds and mammals. While the source of heat may not be reasonably considered an independent character, we see no basis for omitting the behavioral act of incubation as a synapomorphy of birds and mammals (G4a in Table 1). This assumes that the incubation habit of monotremes is the ancestral state for mammals.

(Endothermy is equivocal on the Recent tree (Fig. 2). It either arose in Thecodontia and reversed in crocodiles, or it evolved independently in birds and mammals. The history of this character is unambiguous according to our hypothesis (Fig. 3), which indicates it arose separately in birds and mammals. Similarly, the history of incubation is equivocal on the Recent tree, but unambiguously convergent on ours.)

\section{Similar Thermoregulatory Mechanisms}

Gardiner mentioned (p. 213) several thermoregulatory features in his discussion of endothermy that are shared by birds and mammals. These included protective behaviour (shivering and panting), altering insulation (with erector muscles at the base of feathers and hair) and/or circulation, additional modifiers of heat transfer, a mechanism located in the spinal cord for monitoring the core temperature, the calorigenic effect of certain hormones, non-shivering thermogenesis, and similar behavioral responses to extreme temperatures (e.g., huddling). It is not yet clear at what levels each of these attributes arose in mammals (or for that matter within birds). For example, Carter (1967) pointed out that monotremes have limited thermoregulatory abilities; monotreme body temperatures approach external temperatures at extremes, they have no vasomotor control of skin capillaries, do not pant, and have but a rudimentary ability to sweat. Indeed, shivering appears to be their primary control of internal temperature. Thus, we have not recognized any of these thermoregulatory features as potential synapomorphies of birds and mammals.

It was in the general discussion of thermoregulation that Gardiner argued for a basic similarity in anatomy and development of hairs and feathers, and in doing so he arrived at the novel hypothesis that feathers are modified hairs. He appears correct in noting 
that feathers and hairs possess smooth erector muscles at their bases, and that both structures are induced by special groups of underlying cells (Maderson, 1972; Maderson and Sawyer, 1979). These modifications are not found in other tetrapods, and we consider them bird-mammal synapomorphies (G5a-b in Table 1).

Gardiner made a number of additional claims for resemblances between feathers and hair that we have not been able to verify. For example, hairs appear to be interscale structures (as on a rodent's tail, for example), whereas feathers are not; the feathers growing from scales in hatchling ostriches attest to this (Duerden, 1922). Gardiner also noted the contribution of neural crest to, and continuous growth of, feathers and hair during development, and suggested that these were evolutionary novelties unique to birds and mammals. In the case of the neural crest character, he failed to specify how birds and mammals differed from conditions seen in other amniotes. Further, with the exception of powder down present in some birds, feathers do not grow continuously (Van Tyne and Berger, 1976). Gardiner noted that feathers and hairs are always arranged in groups, or tracts. However, epidermal structures, including scales, are typically arrayed in groups in amniotes, and the tracts seen in mammals and birds display no special correspondences. Gardiner also cited the seasonal changes in plumage and pelt as evidence for Haemothermia, but then temperate zone amniotes typically have seasonal rhythms entrained in their life cycles.

Thus, we do not share Gardiner's opinion regarding the homology of hair and feathers, and the biochemical nature and development of the two structures underscore our opinion. According to Wyld and Brush $(1979,1983)$, alpha keratins are present in the epidermis of all vertebrates. Unlike other vertebrates, however, amniotes have two classes of epidermal keratins, alpha and phi (=beta of some authors), that are distinguished by their high angle X-ray diffraction patterns. The ancestral alpha keratins are retained in the "soft" stratum corneum of all amniotes and in the scales, hairs, and other "hard" structures of mammals. In contrast, al though alpha keratins are retained in softer and more flexible regions of the epidermis of chelonians, birds, crocodiles and lepidosaurs, the "hard" epidermal structures (including feathers) are composed of phi keratins (recorded as G5c in Table 1). Moreover, feathers and hair differ fundamentally in their development. Hairs arise as dermal invaginations (Oster and Alberch, 1982), and according to Northcutt (pers. comm.) they appear to be modified sensory organs not associated with scales. In contrast, feathers, like scales, arise from dermal evaginations; the two are homologous at the level of dermal papillae (Oster and Alberch, 1982).

(Erector muscles and dermal papillae are equivocal on the Recent tree (Fig. 2); they either arose in thecodonts and reversed in crocodiles, or appeared separately in birds and mammals. These characters are unambiguous convergences according to our hypothesis (Fig. 3). Phi keratins arose at the level of Amniota on the Recent tree, and reversed in Mammalia. However, mammals retain the ancestral condition on our tree and Reptilia is diagnosed by having phi keratins.)

\section{Three Meninges}

We found nothing in Stark (1979) to contradict the claim that the ontogenetic division of the endomeninx into an inner pia mater and outer arachnoid layer is an apomorphy peculiar to birds and mammals. Northcutt (pers. comm.) suggested this feature may be a functional correlate of endothermy (character 4 ), because the dense 
connective tissue comprising the "arachnoid layer" is intimately associated with the rich supply of blood vessels passing through the meninges. Further research may help to establish this point; on the basis of current knowledge we tentatively accept Gardiner's interpretation that this character is independent of endothermy. The subdivision of the endomeninx in teleosts, resulting in a total of three meninges (de Beer, 1937: 35), seems to be most simply interpreted as an independently evolved trait.

(On the Recent tree (Fig. 2), an arachnoid layer either arose in thecodonts and reversed in crocodiles, or it evolved independently in birds and mammals. Our hypothesis (Fig. 3) unambiguously supports the latter proposition.)

\section{Folded Cerebellum, Pons Varolit, Inferior Olive and Pontine Nuclei}

According to Northcutt (pers. comm.), Gardiner's description (pp. 213-214) is accurate in that the "cerebellum shows its greatest elaboration in birds and mammals where it is transversely and deeply folded and proportionately larger than the optic lobe." Northcutt also agreed with Gardiner that this character is related to locomotor speed, agility, and overall activity (see also Hopson, 1979, 1980), and that treating them as a character complex is appropriate. For example, inferior and pontine nuclei project from the brainstem to the cerebellum in all vertebrates. One could consider their larger size in mammals and birds to be apomorphic, but then they must be considered redundant with the enlarged and elaborated cerebellum to which they are developmentally and functionally related (Northcutt, pers. comm.). The same argument applies to the prominence of the cerebellar hemispheres and pons varolli, and to the arbor vitae. Northcutt further noted that crocodiles display some development, albeit feeble by comparison to mammals and birds, of lateral hemispheres of the cerebellum. Thus, at least some cerebellar enlargement and elaboration might apply to Gardiner's Thecodontia, while birds and mammals share an even more derived similarity. We have rescored this character to reflect the two derived states (Table 1: G7).

(The elaboration of the cerebellum diagnoses Thecodontia on the Recent tree (Fig. 2); however, its greatest development (state 2) is ambiguous. Our hypothesis (Fig. 3) suggests the archosaur and mammal conditions evolved independently. The ingroup hypothesis of Gauthier (1986; see also Hopson, 1979, 1980) corroborates our position that state 1 gave rise to 2 within ornithosuchians.)

\section{SGroll-Like Turbinals}

Gardiner (p. 214) described birds and mammals as having "prominent, scroll-like turbinals, often supported by bone, and a maxilloturbinal devoid of olfactory epithelium." As discussed below, Gardiner's use of this character presupposed homologies that are doubtful. Moreover, it is clear that "supported by bone" applies only to mammals. So far as we are able to tell from Bellairs and Jenkin (1960), Parsons (1970), King (1979), and Moore (1981), Gardiner was correct in that the primary concha in birds (concha media) and mammals (maxilloturbinal) is prominent and scroll-like, and it is lined with mucociliary respiratory epithelium in both, rather than being at least partly lined with olfactory epithelium as in other amniotes. Accordingly, these resemblances are considered apomorphic (Table 1: G8). Living lissamphibians and turtles (but see below) have no conchae and their states are listed as "not applicable" (N) in Table 1.

Gardiner appears to have missed some other aspects of nasal anatomy which are 
important in amniote phylogeny. For instance, both birds and crocodiles possess an additional conch that lies posterior to the primary concha in a portion of the antorbital space of the cavum nasi proprium (G8a in Table 1). This concha is a simple diverticulum from the lateral wall that is covered by olfactory epithelium (Parsons, 1959, 1970; King, 1979). Parsons (1959) noted that some authors have suggested that the archosaur postconcha and mammalian nasoturbinal are homologous; however, the mammalian nasoturbinal is an elongate, scroll-like lamella arising near the dorsal midline (Moore, 1981), and appears to be related to the ethmoidal system of mammals rather than the archosaur postconcha.

(The presence of scroll-like turbinals is equivocal on the Recent tree (Fig. 2); either it arose in Thecodontia and reversed in crocodiles, or it evolved independently in birds and mammals. According to our hypothesis (Fig. 3), it is unambiguously convergent in birds and mammals. A postconcha diagnoses Archosauria on both trees.)

\section{Maxillary Process}

Gardiner (p. 214) cited "the development of a posteromedially directed maxillary process, termed maxillopalatine in birds (Goodrich, 1930) and alveolar process in mammals (de Beer, 1937)" as a shared apomorphy. We are not sure to what condition of the maxilla Gardiner was referring; strictly speaking it applies to birds only. However, if he meant a broad medial component of the supradental shelf of the maxilla in general, then this character is also present in crocodiles (Iordansky, 1973). The primitive condition is retained by lepidosaurs. A broad shelf may be either absent or present in both pleurodires and cryptodires (Gaffney, 1979), and the former state is tentatively assumed to be ancestral for turtles (Table 1: G9).

(The presence of a broad shelf diagnoses Thecodontia, according to the Recent hypothesis (Fig. 2); however, our tree (Fig. 3) suggests independent evolution within synapsids and archosaurs. Further, the ingroup hypotheses of Gauthier (1986) and Rowe (1986a) indicate at least two independent origins within archosaurs and three within Synapsida.)

\section{Adventitious Cartil.age.}

Gardiner, citing Patterson (1977a), considered the presence of adventitious cartilage diagnostic of birds and mammals. In a recent review of the subject, Hall (1984) concluded that the developmental capacity to produce secondary, or adventitious, cartilage on dermal bones "after the commencement of intramembranous ossification" (p. 156) is absent in all amniotes except mammals and birds. Accordingly, the presence of adventitious cartilage is accepted as apomorphic (Table 1: G10).

(The presence of adventitious cartilage is an ambiguous feature on the Recent tree (Fig. 2). It either arose in thecodonts and reversed in crocodiles, or it evolved independently in birds and mammals. According to our hypothesis (Fig. 3), it is interpreted unambiguously as convergence in birds and mammals.)

\section{Atras, Axis Single Ossifications}

Gardiner (p. 212) claimed that the "condylar articulation with the atlas in birds and mammals differs both from one another and from all other amniotes," and that "the developmental pattern and topographic relationships of this complex is [sic] similar in 
both groups and the atlas and axis are single ossifications." Each of these claims requires some modification.

Huxley (1864) observed that Recent mammals and lissamphibians possess double occipital condyles composed only of exoccipitals, whereas other Recent amniotes possess a single occipital condyle. These elements articulate posteriorly with the atlas intercentrum and neural arches, respectively. Given the information available to Huxley, he correctly interpreted the single condyle as apomorphic. In fact, of the characters Huxley cited as distinguishing his Sauropsida (=turtles + lepidosaurs + crocodiles + birds), this was the only one that could be considered diagnostic, because the rest were ancestral for amniotes. Based on differences in development, de Beer (1937) questioned Huxley's proposed homology between the double occipital condyles of lissamphibians and mammals; the condyles of the former are derived from parts of a more posterior sclerotome that have been added to the skull, whereas those of the latter arise through hypertrophy of the bases of the occipital arch. If they are non-homologous, then one might expect such developmental differences to appear upon closer inspection of the character. However, as de Beer (1937:32) observed in connection with serially homologous structures, a "structure can be transposed up and down the keys in phylogeny without ceasing to play the same 'tune'." Thus, we will consider the double condyles of lissamphibians and mammals to represent the ancestral condition.

Gardiner's second contention is equally unsupported in that the ontogeny of the amniote atlas-axis complex (Gauthier and Rowe, in preparation) reveals no special similarity in the "developmental pattern" shared by birds and mammals. Gardiner was correct, however, in noting that the atlas forms a "single ossification", if he meant that the atlantal neural arches fuse to one another and to the atlas intercentrum in full-grown mammals and birds.

It is unclear to us what Gardiner intended by his statement that mammals and birds share an axis that is a "single ossification." If he meant that the neural arches fuse to one another and to the axial centrum, then he is mistaken, because these structures are fused in all full-grown amniotes. If he was referring to fusion between the axis intercentrum and the atlas centrum, he is again mistaken as to the taxonomic distribution of this character. Such fusion is also characteristic of all extant, fully adult amniotes. Gardiner was correct in noting that the axis centrum and atlas centrum become fused to one another in adult mammals and birds, but not in turtles, crocodiles, or the outgroups. However, this fusion also takes place in full-grown lepidosaurs (Gauthier $e t$ al., 1988a). Thus, we have broadened the taxonomic scope of this character among amniotes and note that it cannot be determined in living lissamphibians.

In summary, reanalysis of Gardiner's claims regarding the development of the occipital condyle and the atlas-axis complex reveals the following apomorphies among extant amniotes. (1) Amniotes do not possess a free axial intercentrum in adults (not recorded in Table 1). (2) Lepidosaurs, mammals, and birds have the atlantal and axial centra fused to one another (G11). (3) The basi-occipital participates in the occipital condyle in turtles, lepidosaurs, crocodiles and birds (G1la). (4) Birds and mammals have a completely co-ossified atlas (G11b).

(The fusion of the atlas and axis centra $($ Gl 1$)$ is equivocal on the Recent tree (Fig. 2); it either arose independently, in lepidosaurs, birds, and mammals, or arose ancestrally in amniotes and reversed in turtles and crocodiles. Our hypothesis (Fig. 3) indicates this character evolved within Synapsida. Further, based on ingroup hypotheses, this character arose within leipdosauromorphs (Gauthier et al., 1988a) and within ornithosuchians 
(Gauthier, 1986). On the Recent tree (Fig. 2), the participation of the baso-occipital in the occipital condyle (G1la) unambiguously arose in Amniota and reversed in Mammalia. Fossil evidence reported by Gauthier et al. (1988b) indicates, however, that the participation is plesiomorphic at the level of Amniota. Our tree (Fig. 3), on the other hand, is consistent with the traditional conclusion, in which the character is restated as loss of participation of the basioccipital in the occipital condyle (but not necessarily complete loss of participation in the craniovertebral joint); this loss took place in extinct relatives of Lissamphibia (Bolt, 1977) and within Synapsida. The completely co-ossified atlas is equivocal on the Recent tree (Fig. 2); it is either diagnostic of Thecodontia and reversed in crocodiles, or has evolved convergently in birds and mammals. Our hypothesis suggests convergence; it arose within synapsids and within ornithosuchians.)

\section{Vascularized Islets of Pancreas}

Gardiner's contention that this apomorphic condition applies only to mammals and birds appears to be correct (Table 1: G12). Bentley (1976:41) described the endocrine pancreas of birds and mammals as "mostly found as vascularized islets free of exocrine tissue." According to Rafn and Wingstrand (1981), the condition in dipnoans, particularly $\mathcal{N}$ eoceratodus, is like the majority of tetrapods, and presumably represents the primitive state.

In connection with the pancreas, we call attention to the position of this gland in crocodiles and birds with respect to the small intestine. These taxa are unique among tetrapods in that the pancreas lies between the limbs of the first duodenal loop, binding them together (Miller and Lagios, 1970; McLelland, 1979). This apomorphy is recorded as G12a in Table 1.

(The history of vascularized islets is ambiguous on the Recent tree (Fig. 2); it either arose in Thecodontia and reversed in crocodiles, or evolved convergently in birds and mammals. Our hypothesis (Fig. 3) suggests unambiguously that this is a case of convergence in birds and mammals. The position of the pancreas with respect to the small intestine, diagnoses Archosauria on both trees.)

\section{Pinealocytes: Oxytocin, Prolactin}

Gardiner (p. 214) stated that in birds and mammals "the pineal ... is modified as an endocrine gland, containing pinealocytes and has an autonomic sympathetic innervation arising from the superior cervical ganglion (Bentley, 1976; Quay, 1979)." Our reading of Quay (1979) suggests otherwise; the pineal part of the parietal eyepineal complex is glandular and contains pinealocytes "with probable endocrine secretory activity" (p. 389) in all amniotes (except in crocodiles in which the complex is absent). Perhaps Gardiner meant to emphasize that in turtles, unlike other amniotes, "only a parenchymatous and presumably mostly non-sensory and active secretory pineal gland remains", and that birds and mammals are further specialized in that "only a compact [in non-passerine and some passerine birds], homogeneous and glandular pineal organ remains" (Quay, 1979: 372). Quay (1979) argued further for a complex developmental history, with each of these groups conscripting various other components of the central nervous system into their pineal glands, thus suggesting convergence in the shared attributes noted above. Of course, Quay's conclusions presume a phylogenetic hypothesis that is here considered the subject of test. Therefore, we follow Gardiner's less theory-laden hypothesis by tentatively accepting the different developmental patterns 
seen in turtles and birds and mammals as specializations superimposed on the apomorphic characters of the pineal gland shared by these taxa (Table 1: G13).

Gardiner's claim that birds and mammals are peculiar among amniotes in having the autonomic sympathetic innervation arise from the superior cervical ganglion requires further study. Our review of Berger and Burnstock (1979) reveals that the autonomic system of crocodiles, lepidosaurs and turtles is too poorly known to enable determination of the level of generality of this character.

Gardiner (p. 214) further argued that birds and mammals are alone among amniotes in "the production of the hormone prolactin." According to Clarke and Bern (1980), however, prolactin constitutes the basic peptide in all vertebrates, apart from lampreys and hagfish. Thus, it is diagnostic of Gnathostomata and the condition in amniotes is primitive.

Gardiner (p. 214), citing Kobayashi and Wada (1973), also stated that mammals and birds are alone among amniotes in "the production of the neurophysial hormone oxytocin". According to Bentley (1976), however, oxytocin is not the neutral peptide in birds; oxytocin is unique to mammals, and birds are like all other choanates in retaining mesotocin.

(Reduction of the visual sensory activity of the pineal gland diagnoses Euamniota and a largely secretory gland characterizes Thecodontia on the Recent tree (Fig. 2). According to our hypothesis (Fig. 3), reduction and loss of the parietal foramen (Table $2,16-17)$ is used to index reduced visual sensory activity, and we hypothesize independent loss within synapsids, testudines and archosauromorphs.)

\section{Magula Densa}

Gardiner (p. 214) claimed that the presence of the "macula densa, a thickening of the distal tubule, which is concerned with the regulation and release of renin (Bentley, 1976)" is a synapomorphy of birds and mammals. The angiotensin-renin system appears to regulate sodium retention and possibly influences blood pressure in all tetrapods. Renin appears to be formed by cells associated with the juxtaglomerular apparatus of the kidney in all tetrapods. So far, only mammals and birds are known to have a macula densa that also "may be concerned with the regulation of the release of renin in mammals (and birds?)" (Bentley, 1976: 57). B. A. Doneen (pers. comm.) cautioned that the avian macula densa is unlike that of mammals, and it has been described as "poorly developed" or "transitional in form". Moreover, other amniotes have been little studied in this respect (Nishimura, 1980). Nevertheless, we tentatively accept the presence of a macula densa in the kidney as an apomorphy shared only by birds and mammals among amniotes (Table 1: G14).

(The thickening of the distal tubule is equivocal on the Recent tree (Fig. 2); either it is characteristic of Thecodontia and reversed in crocodiles, or it is convergent in birds and mammals. The macula densa is convergent on our tree (Fig. 3).)

\section{Loop of Henle}

Gardiner noted that birds and mammals are unique among amniotes in possessing Henle's loop in the kidney (Braun and Dantzler, 1972). Although the loop is relatively poorly developed in birds, it is considered apomorphic of birds and mammals (Table 1: G15).

(The loop of Henle is ambiguous on the Recent tree (Fig. 2); either it arose in 
Thecodontia and reversed in crocodiles, or it appeared convergently in birds and mammals. Our hypothesis is unambiguous (Fig. 3); the loop evolved convergently in birds and mammals.)

\section{LDHX}

Gardiner cited Baldwin and Temple-Smith (1973) as stating that birds and mammals share the apomorphy of a testes-specific LDH known as LDHX. In fact, the subject of the paper by Baldwin and Temple-Smith was to report that although LDHX is known from the testes of therian mammals, it is absent in monotremes. Moreover, Matson (1985; also L. D. Densmore and H. C. Dessauer, pers. comm.) pointed out that LDHX has heretofore been reported only in some pigeons among birds. This electromorph appears to be absent in birds generally, and although the testes of other vertebrates have not been adequately surveyed, it appears to be absent except in some teleosts (M. M. Miyamoto, pers. comm.). Given that the distribution of this apomorphy specifies taxa within mammals and birds, but is general to neither group, we will ignore this character.

\section{Epidermal-MELANin Unit}

Gardiner cited Quevado's (1972) discussion of the epidermal-melanin unit in man (and mouse) as the source of his conclusion that this morphogenetic concept applies only to the system seen in mammals and birds. Quevado's (1972) argument centered on the concept of the epidermal-melanin unit as a structural and functional organization of melanocytes and keratinocytes that exists at levels above those characterizing the individual component cells. Quevado stressed that this unit, rather than the melanocyte alone, serves as the focal point for melanin metabolism within the mammalian epidermis; he made no mention of the situation in birds or other vertebrates. From an earlier work in which conditions in other vertebrates were reviewed (Hadley and Quevado, 1966), it is clear that the epidermal-melanin unit is present in lissamphibians and "some reptiles". The distribution of this character among craniates remains unclear, but current evidence suggests that it was present in amniotes ancestrally, rather than in birds and mammals alone. Therefore, we have dropped this character from our reanalysis.

\section{TheGodont TeEth}

Gardiner (p. 215) cited the presence of "thecodont dentition in which there is a true periodontal membrane between the tooth and alveolus" as an apomorphy shared by crocodiles, birds, and mammals. Unlike other Recent tetrapods, mammals and crocodiles share the apomorphic condition described as a thecodont gomphosis (Osborn, 1984). In keeping with the limitations of this analysis in which evidence from fossils must be ignored, we are forced to consider the conditions in the edentulous living birds and chelonians as indeterminable (Table 1: G18).

(The generality of this character is unclear on the Recent tree (Fig. 2). Thecodont teeth could have arisen either at the level of Euamniota, Thecodontia, or separately in crocodiles and mammals. According to our hypothesis (Fig. 3), it arose unambiguously within synapsids and within archosauromorphs.) 
19. Abolition of Cavum Epiptericum and Incorporation of Epipterygoid into BRAINCASE

Gardiner claimed that the cavum epiptericum is absent in mammals. However, most comparative anatomists would disagree with this assertion. The cavum epiptericum is present in its primitive position lateral to the pila antotica and neurocranium in early mammalian ontogeny, where it contains the ganglion of the trigeminal nerve. Although the cavum becomes incorporated into the completely enclosed cranium, it remains identifiable in adult mammals (Kuhn and Zeller, 1987).

In the same discussion, Gardiner argued that the mammalian alisphenoid is homologous with the laterosphenoid (= pleurosphenoid) of crocodiles and birds. He stated that during crocodilian development there is a membrane extending from an epipterygoid vestige to the braincase, implying that it is the ossification site of the laterosphenoid, because he then claimed that the alisphenoid is thought to have developed in a homologous membrane in Mammalia. However, the laterosphenoid does not ossify in this or any other membrane. The laterosphenoid is an endochondial ossification in the pila antotica, a neurocranial structure that lies medial to the cavum epiptericum and the trigeminal ganglion (de Beer, 1937; Romer, 1956). In contrast, the mammalian alisphenoid is at least in part an ossification in the lamina ascendens of the ala temporalis, which develops from the processus ascendens of the palatoquadrate, a visceral arch derivative. An elongate lamina ascendens occurs in many therians, but only the base or footplate of the ala temporalis develops in monotremes. The adult therian alisphenoid is a compound structure, ossifying endochondrally in the ala temporalis as well as by appositional ossification into the spheno-obturator membrane (Maier, 1987). The processus ascendens of the palatoquadrate also ossifies in lepidosaurs to form the epipterygoid, and in turtles to form a reduced epipterygoid (de Beer, 1937; Maier, 1987). It appears that the mammalian ala temporalis corresponds to the footplate of the lepidosaur and turtle epipterygoid, but that the ascending process of the lepidosaur epipterygoid corresponds to only part of the ascending process of the therian alisphenoid (de Beer, 1937; Maier, 1987; but see Kuhn and Zeller, 1987). In contrast to the pila antotica and the bird-crocodile laterosphenoid, the mammalian alisphenoid and lepidosaur-turtle epipterygoid lie lateral to the cavum epiptericum and its contents. Hence, the elements homologized by Gardiner have different developmental histories and adult relations. Moreover, separate ossifications of both the pila antotica and of the processus ascendens are present in monotremes, and in therians cartilaginous vestiges of the pila antotica are occasionally found together with the large alisphenoid (de Beer, 1937). Put simply, the mammalian alisphenoid and the crocodile-bird laterosphenoid fail the similarity and conjunction tests of homology (Patterson, 1982), and they are therefore not considered part of the same transformation series.

The laterosphenoid is absent in lissamphibians, mammals, turtles and lepidosaurs. Its presence in crocodiles and birds is considered derived, and it is recorded as G19 in Table 1. The epipterygoid is absent in lissamphibians, crocodiles and birds, and therefore this condition is considered ancestral. Its (at least partial) presence in mammals, lepidosaurs and turtles will be considered derived in our reanalysis (Table 1: G19a).

(The laterosphenoid diagnoses Archosauria on the Recent hypothesis (Fig. 2); however, on our tree (Fig. 3) the presence of the bone (Table 2: 71) diagnoses a more general group of archosauromorphs. According to our hypothesis, the presence of an epipterygoid arose prior to the origin of amniotes, and the partial or complete loss of this 
bone has taken place independently in mammals, turtles, crocodiles and birds (e.g., de Beer, 1937; Romer, 1956).)

\section{Completely Divided Heart}

Gardiner intended this character to underscore the similarity of the interventricular septum in crocodiles, birds, and mammals, which have a complete, four-chambered heart. According to Lawson (1979: 497; see also Robertson, 1913), the interventricular septum of lungfishes has a dual embryonic origin. The primary septum originates from an endocardial ridge on the rear wall of the ventricle and grows toward the conus. The secondary septum develops laterally from several muscle buds covered by a thin sheet of endocardium. The buds grow together to form a network of large trabeculae that in turn fuse to the primary septum to form the adult interventricular septum. $A$ vestige of the primary septum may be present in caecilians, and Turner (1967) described a similar ventricular ridge in a salamander. Anurans do not have an interventricular septum and presumably it has been lost. The turtle, squamate, and mammal septum develops like the primary septum of lungfishes. Crocodiles and birds are different in that their interventricular septum, or at least a major portion of it, develops from trabeculae, like the secondary septum of lungfishes (see also Foxon (1955) and Kashyap (1960)). Thus, Lawson's observations contradict Gardiner's statement "that the interventricular septum develops ontogenetically in an identical manner" in both birds and mammals (p. 211; see also Holmes (1975a,b) and Shaner (1962)). Nonetheless, Gardiner was correct in recognizing the complete interventricular septum as a synapomorphy of his Thecodontia (Table 1: G20).

Gardiner also emphasized, but did not explicitly designate as a synapomorphy, the unequal size of the ventricles. He stated that the wall of the left ventricle is thicker and more muscular than the right in birds and mammals. Contrary to Gardiner's interpretation, we believe the bird-mammal state to be much more widespread. For example, Lawson (1979: fig. 11.22) illustrated squamate and anuran hearts with decidedly thicker left ventricles. Moreover, Goodrich (1930: 563, fig. 580) suggested that crocodiles have retained that "primitive condition". In any case, relative thickness of the ventricles appears to be uninformative as regards tetrapod phylogeny and we have not considered that feature in our reanalysis.

Lawson (1979) did indicate, however, that the homeotherm myocardium is thicker and more compact than in other tetrapods, and correlated with that increase is an extensive system of coronary arteries not found in other tetrapods. We have treated such a functionally correlated set of traits as characteristic of birds and mammals (Table 1: G20a).

(A completely divided heart diagnoses Thecodontia on the Recent tree (Fig. 2), whereas we interpret its evolution as one of separate origins-once in archosaurs and once in mammals (Fig. 3). A thick and compact myocardium richly supplied with coronary arteries is ambiguously interpreted on the Recent tree (Fig. 2); it arose in Thecodontia and reversed in crocodiles, or appeared independently in birds and mammals. Our hypothesis (Fig. 3) indicates that it arose convergently in mammals and birds.

\section{Loss of Sinus Venosus and Development of Septum Sinu-venosi}

Gardiner stated (p. 211) that a "complete septum sinu-venosi separates the pre- and 
postcaval openings" in birds and mammals. He also noted the presence of the septum in crocodiles, although he did not describe its size relative to the bird-mammal condition. Goodrich (1930: 556) claimed that a "partial septum sinu-venosi may arise ... [in] ... reptiles"; however, he was not specific as to the degree of development nor the taxa in which it was observed. Kashyap (1960) did not find the septum in the three turtles he studied, but it was generally well-developed among lizards. Simons (1965) noted the presence of a distinct ridge in Sphenodon, which marks the entrance of the left precava into the sinus venosus. It seems that a well-developed septum is much more generally distributed than Gardiner indicated, and we have recorded it as present in all amniotes except turtles (Table 1: G21).

(The loss of the sinus venosus and the development of a sinu-venosi septum is ambiguously interpreted on the Recent tree (Fig. 2), where it originated in amniotes and reversed in turtles, or it appeared in lepidosaurs and thecodonts independently. According to our hypothesis (Fig. 3), this character is also ambiguous; either it appeared in amniotes and reversed in turtles, or it evolved independently in mammals and saurians.)

\section{Complete Diaphragm}

Gardiner used the presence of a "complete diaphragm" separating thoracic and peritoneal cavities to diagnose his Thecodontia. Unfortunately, he did not elaborate on the meaning of "complete", and we are forced to assume he was referring to the partition being imperforate and muscles having fully invaded the septum. The extensive literature on coelomic cavity ontogeny and anatomy reveals considerable complexity often overlooked in more general accounts (see, however, Goodrich, 1930), and we believe that the completely muscularized diaphragm is characteristic of mammals alone.

The ventral portion of the mammalian diaphragm develops from the transverse septum; and the dorsal portion, the oblique septum, originates from the dorsal mesentery and from lateral pleuroperitoneal membranes. The striated muscle of the mammalian diaphragm develops from the hypaxial musculature, more specifically cervical myotomes 3-5 (and perhaps 6 ). It is innervated by the phrenic nerve, which consists of neurons from the ventral rami of cervical nerves $3-5$. The transverse septum is characteristic of Craniata (Goodrich, 1930; Hildebrand, 1974); the oblique septum is found in crocodiles, squamates (but not all lizards and snakes; Bellairs, 1970), birds and mammals (Smith, 1960), and a limited amount of peripheral musculature occurs in or at the margins of the pleuroperitoneal septum of some turtles, crocodiles, and birds (Wiedersheim, 1897). The septum is not only nearly devoid of muscle in most nonmammalian amniotes but it is often perforate (even in birds). The crocodilian diaphragmatic muscle, which is attached to the abdominal sternal plates, is innervated by abdominal spinal nerves (Porter, 1972). Thus, we find little evidence in comparative anatomy and ontogeny for a "complete diaphragm" in crocodiles, birds and mammals.

Other variation in coelomic septa might be informative in delimiting major groups of amniotes. For example, Butler $(1889,1892)$ recorded a posthepatic septum in crocodiles and birds. It develops from the falciform and gastrohepatoduodenal ligaments of the ventral mesentery system. Striated muscle fibers from the posterior body wall invade this connective tissue partition and they are innervated by lumbar spinal nerves, not by phrenics (Ballard, 1964). The posthepatic septum is observed elsewhere among some squamates, such as the teiid Tupinambis and snakes, but not in Sphenodon, and thus seems 
most simply interpreted as convergence (Goodrich, 1930). In fact, the teiid septum develops from the gastrohepatic ligament and it contains smooth muscle fibers. We have employed the bird-crocodile posthepatic septum in our revised data matrix (Table 1: G22a).

(A posthepatic septum diagnoses archosaurs on both trees (Figs 2, 3).)

\section{External Auditory Meatus and Valve}

Gardiner (p. 216) concluded that a "considerable external auditory meatus... [with] ... a movable integumentary valve or pinna" is a synapomorphy uniting birds, mammals, and crocodiles. Although the structures differ in several aspects of form and development, they do share certain apomorphies, such as being overhung by the squamosal (see Wever, 1978), and a "considerable external auditory meatus" will therefore be considered apomorphic for birds, crocodiles, and mammals (Table 1: G23). Some squamates also have a deep meatus, but we interpret this as secondary because Sphenodon and most squamates have no such structure. The movable integumentary valve to which Gardiner referred is diagnostic of crocodiles only, as is the pinna of therians only, and neither condition is present in birds, so they will not be considered in our reanalysis.

(An elongate external auditory meatus diagnoses Thecodontia on the Recent tree (Fig. 2), whereas it appears convergently on our hypothesis (Fig. 3), once within synapsids, and once in archosaurs. Evidence for a meatus in archosaurs, aside from crocodiles and birds, is difficult to determine. Although we list this character as diagnostic of $A$ rchosauria, further research may indicate convergence within the group.)

\section{Three Turbinais}

Gardiner described the presence of three turbinals as a synapomorphy uniting crocodiles, birds and mammals. Once again, this assertion requires more careful consideration than that provided by Gardiner, who in any case offered no citation that would substantiate such a claim. Following Parsons (1959, 1970), Gardiner's term turbinal will be restricted to the bony lamina in the cavum nasi propium. $\Lambda$ s such, the term turbinal will be considered a subset of the more general term concha, which applies to any projection of the lateral wall of the cartilaginous nasal capsule into the cavum nasi propium.

Nasal conchae are said to be absent in amphibians (Jurgens, 1971) and dipnoans (Bertmar, 1965), and these projections from the lateral wall into the cavum appear to be present in all amniotes, with the possible exception of turtles. The discovery of conchal synapomorphies among amniotes is complicated by two factors, the diversity of adult morphologies and the dearth of comparative embryological studies. Parsons (1959:244) noted that the "problems of conchal homologies among the various amniotes have received much attention in the literature, but there is, as yet, no general agreement". Gardiner's assertion suggests that he has solved this long-standing controversy; it is true that turtles have either one concha, or none (see below), Sphenodon has two, squamates have one (or none, see Stebbins, 1948), and birds, crocodiles and mammals have three (Parsons, 1959). As soon as one considers the ontogeny and adult morphology of amniote conchae, however, the inadequacy of Gardiner's treatment of this variation becomes apparent. For example, the most anterior concha in birds arises in the wall of 
the vestibulum, and not in the cavum nasi proprium as do the remaining conchae of birds and those of all other amniotes (Parsons, 1959). Likewise, the middle (primary concha) and anterior (preconcha) conchae of crocodiles arise from a single embryonic concha, presumably the primary concha, that later divides into two, unlike any other amniote (Parsons, 1959). Thus, Gardiner's use of "development of three turbinals" (p. 216) to unite birds, crocodiles, and mammals is both inadequate and misleading, and we have ignored this character.

Parsons (1959, and subsequent reviews) considered conchae to be absent in turtles, but present in all other amniotes. Thus, like the supposed absence of "Jacobson's organ" in turtles, he considered the supposed absence of a concha to be another character that might distinguish turtles from all other amniotes. Parsons argued that the only homology among amniote conchae for which there is evidence of more than superficial similarity is that of the primary concha (=maxilloturbinal in mammals, concha posterior in Sphenodon, concha in squamates, preconcha + concha in crocodiles, and concha media in birds). This concha is considered homologous in that it is the first to appear in ontogeny in all groups, and at its initial appearance it is often a broad, hemispherical eminence projecting from the lateral wall into the cavum nasi proprium. In turtles, both the so-called lateral Grenzfalte and a more posterodorsal eminence, the Muschelwulst, with which the lateral Grenzfalte is continuous, have been suggested to be homologous with the primary concha of other amniotes (Gegenbaur, 1873; Seydel, 1896; Fuchs, 1915). These structures are most prominent in terrestrial turtles (e.g., Testudo); but the Muschelwulst in particular, and to a lesser extent the lateral Grenzfalte, tend to be feebly developed or absent in emydid turtles (Seydel, 1896; Parsons, 1959, 1970). Parsons (1959) noted that the lateral Grenzfalte appears early in ontogeny and occupies a position near a primary concha, as might be expected. However, he thought the two were unlikely to be homologous owing to differences in shape. Nevertheless, the similar position and temporal appearance in ontogeny, combined with the fact that the bulk of the olfactory epithelium lies dorsal to these structures in all amniotes, and bearing in mind that conchae are absent in outgroups, indicate that we are dealing with a concha, albeit of a sort peculiar to turtles. We are struck by the similarity of the nasal capsules of some turtles and that of Sphenodon, as both possess relatively unmodified nasal capsules compared to those of other amniotes (compare Chelydra, fig. 1B, Emydoidea, fig. 2B, and Tesiudo, fig. 4B, with Sphenodon, fig. 12, in Parsons, 1970). Comparisons of early ontogenetic stages of those turtles possessing more prominent Muschelwulst-lateral Grenzfalte, such as Testudo, Kinosternon, and Chelydra, to similar developmental stages of Sphenodon should be made, because Parsons (1959) rejected the homology of these structures on the basis of his studies of emydids, in which the eminence is poorly developed.

We recognize that there are problems with our suggested homology of the primary concha across all amniotes. These eminences vary among cryptodires, and few pleurodires appear to have been surveyed for this character. Moreover, even in turtles where the Muschelwulst-lateral Grenzfalte is prominent, it does not approach the degree of development seen in other amniotes, nor is it particularly similar in shape. It must be borne in mind, however, that without an outgroup upon which to root possible transformation series, one is unable to decide which of the two shapes represents the ancestral condition. We think it likely that a primary concha was present in the ancestral amniote. However, we yield to Parsons' (and by extension, Gaffney's, 1980) interpretation in the present study in order to avoid placing undue weight on our 
speculations. Thus, conchae are scored as absent in turtles and present in all other amniotes (G24a in Table 1).

(Given that we score nasal conchae as absent in turtles, the history of these structures is equivocal according to both hypotheses (Figs 2,3). Conchae either evolved in ancestral amniotes and reversed in turtles, or appeared separately in lepidosaurs and in thecodonts (Fig. 2), or in mammals and in saurians (Fig. 3). As our review of this character indicates, however, we believe that the presence of nasal conchae is an amniote character, which has been lost secondarily only in some turtles.)

\section{Renal Portal System}

Gardiner's use of "a renal portal system (rudimentary in crocodiles, birds and monotremes)" to diagnose Thecodontia is misleading, because it gives the impression that the system is present in a complete form in therian mammals. $\Lambda$ s suggested by Løvtrup (p. 465), the opposite is true. Apparently Gardiner was emphasizing the tendency to lose the renal portal system in crocodiles, birds, and mammals as the derived state; a well-developed portal system is assumed to be ancestral, because it is at least as generally distributed as Gnathostomata (see Løvtrup). In addition, we believe the reductions of the renal portal system in crocodiles and birds and the loss in mammals are not parts of the same transformation series and we have scored them as different characters (the mammalian autapomorphy has been omitted from Table 1: G25). The loss of the renal portal system is correlated with the elaboration of other vessels that carry venous blood directly to the heart, and our interpretation of independent evolution is based on the strikingly different ways kidney-bypass develops (Smith, 1960). The capillary beds between the renal portal and renal veins in crocodiles and birds are reduced and there exists a separate, direct, noncapillary connection between the renal portal vein and the main venous trunk leading to the heart. The bypass develops symmetrically and involves a new vessel, the great renal vein, plus the subcardinal (Nelsen, 1953). However, in mammals, including monotremes (Barone, 1972), with the loss of the portal system, a direct connection to the main venous trunk leading to the heart develops asymmetrically from the right supracardinal and subcardinal veins (Patten, 1952). There is no evidence that the great renal and supracardinal veins are the same vessels.

(The loss of a renal system, involving evolution of a bypass via a great renal vein and subcardinal, diagnoses Archosauria in both classifications (Figs 2, 3).)

\section{Pneumatization of Middle Ear}

Gardiner (p. 216) correctly described crocodiles and birds as being apomorphic in that they have numerous extensions "of the middle ear air-space [into] ... the basioccipital, parasphenoid, quadrate and articular." He also noted that "in mammals the diverticula of the tympanic cavity are confined to the mastoid process" (p. 216). A pneumatic mastoid may represent the ancestral condition for mammals (Rowe, 1988), although we note that diverticula of the cavum tympani are reported to invade the supra-occipital in multituberculates (Kielan-Jaworowska et al., 1986), and within placentals to the squamosal, among other bones (e.g., Kampen, 1905: 341; Werner, 1960). Although it is true that birds, crocodiles and mammals resemble each other in having pneumatic extensions of the cavum tympani, the cavities penetrate entirely different bones in birds and crocodiles on the one hand, and mammals ancestrally on the 
other. In keeping with the criteria established at the outset (see the section "Data and Methods of Analysis"), we recognize only the derived correspondence of the bird-crocodile pneumatization of the basioccipital, parasphenoid, quadrate and articular in Table 1 (G26).

(Pneumatization of the middle ear diagnoses Archosauria according to both trees (Figs 2, 3). However, pneumatization may in fact be convergent within archosaurs, because it is only observed in crocodylomorph pseudosuchians (Martin, 1983) and some coelurosaur theropods (Currie, 1985). It also evolved convergently in the mammalian mastoid (prootic + opisthotic), and spread to adjacent bones within mammals.)

\section{Perilymphatic Dugt and Coiled Coghlea: Canalis Reuniens; Membrana TECTORIA}

Gardiner (p. 216) argued that birds, mammals, and crocodiles are peculiar among amniotes in that "the perilymphatic duct is expanded into a double loop and the lagena forms a pronounced cochlear duct". He provided no supporting references, and we have been unable to verify that the perilymphatic duct has been expanded into a "double loop" in any vertebrate. Gardiner, however, was correct in noting that an elongate cochlea is present in crocodilians (Wever, 1978), and in birds and mammals (Pumphrey, 1961). The cochlea is short in other amniotes (Wever, 1978). We recognize this character as G27 in Table 1.

Gardiner (p. 216) cited Berg (1926) as the source for his conclusion that in birds, crocodiles, and mammals, but not in other amniotes, the "cavity of the membranous cochlea is united with the sacculus by means of a narrow canalis reuniens and a membrane of Corti (membrane tectoria) covers the auditory cells". $\Lambda$ s is evident from more recent studies (e.g. Baird, 1970; Wever, 1978), however, a tectorial membrane also overlies the auditory cells in turtles and lepidosaurs, and they are also like other amniotes in that a narrow canalis reuniens (= sacculocochlear duct) connects the sacculus and cochlea. Since these conditions obtain in all amniotes, they cannot constitute evidence for Gardiner's Thecodontia.

In researching Gardiner's inner ear characters, we discovered that the "crocodilian form of Reissner's membrane appears to take an intermediate position between the simple form seen in most reptiles... [and mammals] ... and the elaborately convoluted form occurring in birds" (Wever, 1978: 78). Specifically, crocodiles are like birds, and unlike other amniotes, in that Reissner's membrane, which appears to be involved in endolymph secretion, "becomes greatly thickened at its medial edge, especially toward the dorsal end of the cochlear duct, and then more dorsally still this portion of the membrane is thrown into numerous folds (Wever, 1978: 78)." We have employed this character in our reanalysis (Table 1: G27a).

Gardiner (p. 214) noted that birds and crocodiles are alone among amniotes in having the eustachian tube pass "through the cranial base to open by a single opening in the roof of the pharynx". He cited no references supporting his claim that the condition arose convergently, but his conclusion of shared apomorphic resemblance is acknowledged by Wever's (1978) description of crocodiles and Bellairs' and Jenkin's (1960) description of birds. We have also employed this character in our reanalysis (Table 1: G27b).

(The presence of an elongate cochlea diagnoses Gardiner's Thecodontia (Fig. 2); however, our hypothesis (Fig. 3) suggests that it evolved convergently in mammals and archosaurs. We doubt that cochlear elongation is ancestral for archosaurs, because the 
early members of both major groups, Pseudosuchia and Ornithosuchia, do not appear to exhibit this condition (e.g., Camp, 1930). The morphology of Reissner's membrane and the eustachian system diagnose Archosauria in both Gardiner's and our taxonomies (Figs 2, 3).)

\section{Epipterygoid Joins Parietal}

Gardiner's hypothesis of homology of the laterosphenoid-alisphenoid-epipterygoid is necessary to his proposal that the parietal contacting the epipterygoid is a synapomorphy of turtles, crocodiles, birds and mammals. However, as discussed above (see G19), the laterosphenoid of birds and crocodiles bears little similarity to the mammalian alisphenoid or the lepidosaur-chelonian epipterygoid. The structures in question have different ontogenetic histories and adult anatomical relations, and we agree with the long-recognized opinion that they are not homologous (e.g., de Beer, 1937; Maier, 1987). Thus, we discard this character.

\section{Stapes Unites with Megkel's Gartilage}

Gardiner concluded that the stapes is connected via the pars interhyales to Meckel's cartilage in mammals, birds, crocodiles, and turtles, but not in lepidosaurs. However, according to de Beer (1937; see also Bellairs and Kamal, 1981), the interhyal persists as a ligament in some adult squamates and remains chondrified throughout life in Sphenodon. Taking into account this more general distribution, we consider the trait an amniote synapomorphy and omit it from our reanalysis.

\section{Penis Single and with Eregtile Tissue}

Gardiner stated that a single erectile penis is characteristic of turtles, crocodiles, birds, and mammals. Tonutti $(1931,1932,1933)$ hypothesized that the amniote penis evolved from the caecilian phallodeum. Wake's (1972) analysis indicates otherwise. The caecilian intromittent organ is unusual in many respects. It arises from an outgrowth of the rear part of the cloaca, rather than from a genital tubercle, as it does in amniotes (M. H. Wake, pers. comm.). If 'Tonutti's hypothesis is correct, the penis is a tetrapod synapomorphy and the organ has been lost independently at least three times (anurans and caudates, neognath birds, and lepidosaurs). However, the absence of the penis in dipnoans, anurans, and caudates, and the fact that the functionally similar organ in caecilians bears no morphological similarity to that in amniotes, suggest an alternative interpretation: the caecilian and amniote single, median organs are independently evolved, and frogs and salamanders retain the ancestral condition. Under this hypothesis, which we adopt, the lepidosaur condition is treated as primitive. There appears to be no basis in anatomy or ontogeny for considering the hemipenes of squamates as homologues of the penis found in turtles, crocodiles, birds, and mammals (Raynaud and Pieau, 1985). Sphenodon, the sister group of squamate lepidosaurs, has no copulatory organ (Table 1: G30).

(A single erectile penis diagnoses Gardiner's Euamniota (Fig. 2); however, our hypothesis (Fig. 3) indicates that this character was present in amniotes ancestrally and was subsequently lost in lepidosaurs; the lepidosaur condition is correlated with the transformation of the cloacal slit from an anteroposterior to a transverse orientation (Gauthier et al., 1988a).) 


\section{Solid Ovaries}

Gardiner used solid ovaries to diagnose his Euamniota (Fig. 1); however, this conclusion is mistaken, because the ovary in Sphenodon is solid and like that of turtles, crocodiles, birds, and mammals (Porter, 1972: 170). While it is true that squamates have a saccular type of ovary (Porter, 1972), the adult morphology and developmental history of those cavities is quite different from the primitive anamniote condition (Nelsen, 1953; Fox, 1977; Raynaud and Pieau, 1985). Thus, we omit this character from our reanalysis, because it does not help to resolve relationships among the extant terminal taxa we consider.

\section{Giliary Processes}

Gardiner stated that ciliary processes, fin-like structures on the inner, anterior surface of the ciliary body, occur in turtles, crocodiles, birds, and mammals. However, Walls (1942) noted their presence in tetrapods generally. According to Walls (1942: 267), comparable structures in living amphibians, while small, deserve to be called ciliary processes. However, M. H. Wake (pers. comm.) pointed out that ciliary processes are absent in caecilians. Ciliary processes were not observed in snakes, but Walls thought they were present in other squamates, if only in a reduced form. However, Underwood (1970: 82) stated that, as in Sphenodon, the processes are absent in all squamates. Following Walls, we will consider small ciliary processes the ancestral condition in Tetrapoda, and the well-developed ciliary processes of mammals, turtles, birds, and crocodiles as apomorphic (Table 1: G32).

(According to the Recent tree (Fig. 2), well-developed ciliary processes diagnoses Euamniota. The processes become enlarged in early amniotes and secondarily reduced or lost in lepidosaurs in our hypothesis (Fig. 3).)

\section{Eyelid Cartilage}

We have been unable to understand what Gardiner meant by "eyelid cartilage" present in mammals, birds, crocodiles, and turtles (his fig. 2). His only mention of this character in the text was in the statement (p. 217) "the levator muscle of the upper eyelid is supported by a lid cartilage which may be ossified". First, firm plates (so-called tarsi which are composed of dense connective tissue and may be ossified) are found in either or both lids, but they occur between the muscle sheets and their conjunctival linings. Morever, according to Walls (1942) and Underwood (1970), tarsi are (1) absent in amphibians and turtles, (2) usually present in the lower, but not the upper, lid in lepidosaurs and birds, (3) absent in the lower lid but present in the upper lid of crocodiles, usually as a bony element, and (4) usually, but not always present in both lids in mammals. Crocodiles also possess bones above the orbit, which may be only loosely associated with the skull. These bones, palpebrals, are comparable to the supra-orbital elements (true osteoderms) of some squamates in that they are formed in the dermis of the upper eyelid (Romer, 1956); however, they are anatomically and ontogenetically unlike tarsi. The taxonomic distribution of tarsi bears no resemblance to that described by Gardiner, and the variability of tarsi within the terminal taxa and between the upper and lower lids, and the questionable comparability of tarsi to palpebrals and supraorbital bones leaves us no other choice but to abandon the character. 


\section{Tendon of Nigtitans to Pyramidalis Muscle}

According to Gardiner (p. 217), the tendon of the nictitans (= nictitating membrane) stretches from the orbital wall to the pyramidalis muscle on the surface of the eye in turtles, crocodiles, and birds; that state was also attributed to mammals in his summary cladogram (Fig. 1). The tendon in question is absent in amphibians and mammals (Walls, 1942: 426), and the pyramidalis muscle is present only in turtles, crocodiles, and birds. The pyramidalis is absent in mammals, even in those with a well-developed nictitating membrane (Walls, 1942: 38, 426). Accordingly, we have restated a portion of this character as the absence (ancestral) or presence (derived) of the pyramidalis muscle alone; the derived condition is known only in turtles, crocodiles, and birds (Table 1: G34). Underwood (1970: 82) proposed that "the pyramidalis is derived from fibres of the lateral retractor bulbi muscle that already had an association with both the eyeball and the tendon." Both the retractor and the pyramidalis are innervated by the abducens nerve.

It is unclear if the ancestral point of attachment of the tendon of the nictitating membrane is to the surface of the eye (turtles, crocodiles, and birds) or to the interorbital septum (lepidosaurs), because the tendon is absent in the outgroups. $\Lambda$ ccordingly, these character states are treated as unordered in the reanalysis (Table 1: G34a).

We discovered three additional synapomorphies among amniotes in Walls' (1942) book on the vertebrate eye. First, there exists a large and highly mobile nictitans in turtles, crocodiles, birds, and lepidosaurs. In mammals, at least in the cladistically primitive members of that taxon, the nictitans is small and immobile (the so-called haw). The nictitans is absent in amphibians, an even less derived condition. We have recorded this character as G34b in Table 1 . In addition, the tendon involved in moving the lower eyelid in amphibians and turtles is absent in mammals, birds, crocodiles, and lepidosaurs (Table 1: G34c). The third character, a transparent nictitans, is diagnostic only in crocodiles and birds (Table 1: G34d).

(The history of the pyramidalis muscle is ambiguous on both trees. On the Recent tree (Fig. 2), it is either diagnostic of Euamniota and reversed in mammals, or it arose separately in turtles and archosaurs. Similarly, we could hypothesize that it either arose in ancestral reptiles and reversed in lepidosaurs, or that it evolved independently in turtles and archosaurs (Fig. 3). The history of the insertion of the amniote nictitans tendon is equally difficult to interpret, because the tendon is absent in mammals and the outgroups (but see Underwood, 1970). On the Recent tree (Fig. 2), an insertion on the eyeball via the pyramidalis could diagnose Amniota, with the lepidosaur state being an autapomorphy. Alternatively, the lepidosaur condition could be ancestral, with a subsequent shift to the pyramidalis diagnosing Euamniota. In either scenario, the absence of a tendon in mammals would be interpreted as a loss. According to our hypothesis (Fig. 3), this absence is considered a plesiomorphy, with the appearance of a tendon for the nictitans diagnosing Reptilia. We cannot, however, determine if the primitive attachment of the tendon in reptiles was to the interorbital septum or to the eyeball via the pyramidalis.

The presence of a nictitans diagnoses Amniota on the Recent tree (Fig. 2), with the feeble condition in mammals being a reversal. Alternatively, our hypothesis (Fig. 3) suggests that a feebly developed nictitans first appeared in ancestral amniotes and subsequently became well-developed (and acquired its own tendon) in reptiles. The loss of the tendon to the lower eyelid is equivocal on the Recent tree (Fig. 2); it could have 
been lost in amniotes ancestrally, subsequently re-evolving in turtles, or it could have been lost independently in lepidosaurs and thecodonts. The history of this character is equivocal on our tree as well (Fig. 3). The tendon could have been lost in amniotes ancestrally, and re-evolved in turtles, or it could have been lost independently in mammals and saurians. Accessory evidence in Walls (1942) suggests that the latter may have been the case, because "loss" of the lower eyelid tendon does not appear to represent comparable apomorphies among amniotes (but see Underwood, 1970, for an alternative view). That is to say, lissamphibians and turtles are unable to close the lower lid without retracting the eyeball (the eyeball retracts the tendon, thus passively raising the lower lid). In contrast, mammals and saurians are able to close their eyelids without retracting the eyeball by virtue of intrinsic eyelid muscles. The intrinsic system in mammals is unique because it is derived from the facial platysma peculiar to that group. The system in saurians is unlike the condition in mammals in that no platysma, or derivatives, occur in the former taxon. These muscles should be studied more in saurians with a view to determining possible homologies. The appearance of a transparent nictitans is diagnostic of archosaurs in both hypotheses (Figs 2, 3).)

\section{Thyroid and Cricoid Cartilages}

Gardiner listed thyroid and cricoid cartilages as characteristic of all amniotes but lepidosaurs. However, according to Romer (1956), Smith (1960), and Wiedersheim (1897), the cricoid is also found in amphibians and lepidosaurs, whereas the thyroid is unique to mammals. Thus, neither cartilage is informative in establishing amniote relationships, and we have omitted these characters.

Edgeworth (1935) argued that in contrast to amphibians and mammals, the cricoid is composed of two or more tracheal rings in turtles, lepidosaurs, crocodiles, and birds. This variation is recorded as G35a in Table 1 .

(On Gardiner's tree (Fig. 2), a cricoid composed of two or more tracheal rings diagnoses Amniota and reverses in mammals. According to our hypothesis (Fig. 3), mammals retain the ancestral condition, and this synapomorphy diagnoses Reptilia.)

\section{Cartilaginous Cones at Ends of Longbones}

Gardiner (p. 217) stated that the presence of "temporary, massive cartilaginous cones ... at each end of the shaft of the long bones" is a synapomorphy of turtles, crocodiles, and birds. Gardiner provided no citation for this statement, but it seems likely that it was based on Haines' (1969) review. Our study of the growth of tetrapod longbones and review of the literature, however, does not support Haines' (1969) conclusion that cartilage cones are unique to birds, crocodiles, and turtles. Since the diameter of the shaft increases towards the articular surfaces at either end, and the cylinder of periosteal ossification spreads from the mid-diaphysis outward in all tetrapods, the appearance of "temporary cartilage cones" is but an artifact of the geometry of the cartilage model and the manner of ossification of longbones. The apomorphic condition noted by Haines (1969) is the appearance of independent centers of ossification in the proximal end of the tibia in birds and in the epiphyses of all longbones in mammals and lepidosaurs. We have included this three-state character in our reanalysis (Table 1: G36a).

Gardiner (p. 217) stated that “... specific 'primary osteones' occur in the development of the bone of turtles, crocodiles and young mammals." His authority for this conclusion was Enlow (1969: 55), who noted that during "periods of particularly 
rapid growth, a pattern of bony tissue is produced in crocodilians and turtles that parallels a common type also formed in the fetal and very young postnatal mammalian skeleton." Enlow (1969: 57) referred to this pattern of bony tissue as a primary osteone "in order to distinguish it from typical Haversian systems or secondary osteones" (Enlow, 1963). However, a few pages later, Enlow (1969:62) noted that "young, rapidly growing lizards and snakes have not yet been studied extensively, and bones from a complete age series have never been examined." Thus, although Enlow did not report primary osteones in lepidosaurs, this conclusion was based entirely on negative evidence. Moreover, Enlow did not discuss the condition in either birds or amphibians. Consequently, without more complete information Gardiner's conclusion of synapomorphic resemblance in the possession of primary osteones in rapidly growing turtles, crocodiles and mammals, appears premature.

(A secondary ossification center in the proximal end of the tibia is equivocal on the Recent tree (Fig. 2); it could have evolved in amniotes ancestrally, reversing in turtles and crocodiles, or it could have appeared independently in lepidosaurs, mammals, and birds. Based on the ingroup hypothesis of relationships within ornithosuchians (Gauthier, 1984, 1986), the cnemial epiphysis is considered a synapomorphy of ornithurine birds only. Moreover, secondary ossification centers are ancestral for lepidosaurs, but not for lepidosauromorphs (Gauthier et al., 1988a). In our hypothesis (Fig. 3), such ossifications evolved convergently in Mammalia and within lepidosauromorphs, and are not homologous with the cnemial epiphysis of some birds.)

\section{Subclavian Arteries Displaced Gephalad}

Gardiner's use (p. 217) of the "cephalad displacement of the subclavian arteries during development" to diagnose Euamniota is difficult to evaluate in the absence of more specific information on both anatomical details and references. It is not clear whether "development" of the vessels is important or whether the position in the adult alone is what Gardiner was referencing. Another basic problem concerns whether he was referring to the primary subclavian (a segmental artery which degenerates during development) or the permanent or secondary subclavian which develops later in amniote ontogeny at or near the third aortic arch (Manner, 1964; Nelsen, 1953). The primary and permanent subclavians are equivalent in name only, because both may occur in the same individual (e.g., a bird; Nelsen, 1953). If the permanent subclavian is the vessel in question, then it is also important to know whether Gardiner was specifying the right or left artery because there is considerable asymmetry of origin in amniotes (Smith, 1960). If we assume Gardiner was referring to some average position of the adult permanent subclavians, then we can confirm (Holmes, 1975b) that the vessel(s) has a far anterior point of origin, at or near the base of the carotids (the third arch), in crocodiles, birds, and mammals. However, turtles are not like crocodiles, birds and mammals, because both subclavians come off the right aorta (arch four), near the heart. Both lepidosaur subclavians (Beddard, 1905; O'Donoghue, 1921) also originate from the right aorta, but more posteriorly (Holmes, 1975b).

At the risk of oversimplifying the pattern of permanent subclavian variation in tetrapods, we recognize four states. We assume that one of the several dipnoanlissamphibian conditions is ancestral (Goodrich, 1930): a symmetrical posterior origin from either the single or paired dorsal aortae, like that in Neoceratodus or frogs generally, or one in which primary, but not permanent, subclavians are present, as is typical of 
salamanders. The subclavians cannot be distinguished from the segmentally arranged vertebral arteries in caecilians (Lawson, 1970; Ramaswami, 1944), as might be expected in limbless tetrapods. In any case, all amniotes are derived, to the extent that there has been a shift craniad in the point of origin of the subclavians from the right or left third or fourth aortic arches. The conditions in turtles and lepidosaurs are similar and these are recognized as the same state. The subclavians are located farther anteriorly, nearer the carotids, in crocodiles, birds, and mammals. We further recognize the subclavian condition in birds and crocodiles as another state (Jollie, 1962), in which the vessel originates anterior to the separation of the internal and external carotids, not posterior to it as in mammals. None of the amniote conditions are present in the outgroups, and this complicated multistate character is scored as unordered (Table 1: G37).

(Both the Recent tree and our hypothesis (Figs 2,3) indicate that Amniota is diagnosed by an anterior shift in the position of the subclavians. Thecodontia on the Recent tree is delimited by a further cranial shift of those vessels. According to our cladogram, the positions in mammals and archosaurs evolved independently. The most anterior position of the subclavians diagnoses Archosauria on both hypotheses.)

\section{Lovtrup's Data}

\section{Histology of Neurohypophysis}

Lovtrup (table 1: 18) cited Wingstrand's (1951) classic work on the structure and development of the pituitary gland as indicating apomorphic resemblances in the histology of the neurohypophysis in mammals and birds. However, our interpretation of Wingstrand's account of this region (pp. 237-350) differs. Wingstrand (p. 239) began his discussion by noting that the "histological structure of the neurohypophysis was said to vary extensively in birds, and the same holds good for amniotes in general." Nevertheless, he stated (p. 242) that the "primitive type of neurohypophysis found in Phasianus, Gallus, Diomedea and owls may be directly compared with the type found in Sphenodon and many lizards, whereas the advanced type found in Anser, Larus, etc. is similar to that of snakes." He went on to describe the mammalian neurohypophysis ( $p$. 242) as being "invariably of a more complicated type." His description of monotreme neurohypophysial morphology showed, however, that they retain most of the ancestral amniote conditions; a "much more complicated" neurohypophysis appears to be diagnostic of therian mammals. Wingstrand made many comparisons between mammals and birds in this and other sections of his monograph. However, in the absence of explicit descriptions of conditions in other amniotes and outgroups, it would be premature to consider any particular point of similarity as representing an apomorphic condition. For example, he pointed to several similarities in the histology of the avian and mammalian adenohypophysis, and compared and contrasted them with the highly variable conditions in squamates. However, as he noted (p. 149), little can be made of these similarities because "no histological comparison [has been] made with the Chelonia, Crocodilia and Rhynchocephalia, [because] the literature reports are too old and incomplete and my own material too scanty." Accordingly, we have discarded this character from our reanalysis.

Pearson (1985: 714) stated that the "hypophyseal stalk forms at the anterior end of the anlage, and the lateral lobes, described in both groups [crocodiles and birds] as stringlike, develop in a similar manner." She also reported (p. 714) that crocodiles and birds 
Table 1

Synapomorphies of the major groups of living amniotes.

\begin{tabular}{|c|c|c|c|c|c|c|}
\hline & Ancestor & Lepidosauria & Chelonia & Crocodylia & Aves & Mammalia \\
\hline G2 & 0 & 0 & 0 & 0 & 1 & 1 \\
\hline G3 & 0 & 0 & 0 & 1 & 1 & 1 \\
\hline G3a & 0 & 0 & 0 & 1 & 1 & 0 \\
\hline G4 & 0 & 0 & 0 & 0 & 1 & 1 \\
\hline G4a & 0 & 0 & 0 & 0 & 1 & l \\
\hline G5a & 0 & 0 & 0 & 0 & 1 & l \\
\hline $\mathrm{G} 5 \mathrm{~b}$ & 0 & 0 & 0 & 0 & l & 1 \\
\hline G5r. & 0 & 1 & 1 & l & 1 & 0 \\
\hline G6 & 0 & 0 & 0 & 0 & 1 & 1 \\
\hline G.7 & 0 & 0 & 0 & 1 & 2 & 2 \\
\hline G8 & $\mathrm{N}$ & 1 & $N$ & 1 & 2 & 2 \\
\hline G8a & 0 & 0 & 0 & 1 & 1 & 0 \\
\hline G9 & 0 & 0 & 0 & 1 & 1 & 1 \\
\hline G10 & 0 & 0 & 0 & 0 & l & 1 \\
\hline G11 & $\mathrm{N}$ & 2 & 1 & 1 & 2 & 2 \\
\hline Glla & 0 & 1 & 1 & 1 & 1 & 0 \\
\hline Gl1b & 0 & 0 & 0 & 0 & 1 & 1 \\
\hline G12 & 0 & 0 & 0 & 0 & 1 & 1 \\
\hline G12a & 0 & 0 & 0 & 1 & 1 & 0 \\
\hline G13 & 0 & 0 & 1 & $\mathrm{~N}$ & 2 & 2 \\
\hline G14 & 0 & 0 & 0 & 0 & 1 & 1 \\
\hline G15 & 0 & 0 & 0 & 0 & 1 & 1 \\
\hline G18 & 0 & 0 & $\mathrm{~N}$ & 1 & $\mathbf{N}$ & 1 \\
\hline G19 & 0 & 0 & 0 & 1 & 1 & 0 \\
\hline G19a & 0 & 1 & I & 0 & 0 & 1 \\
\hline G20 & 0 & 0 & 0 & 1 & 1 & 1 \\
\hline G20a & 0 & 0 & 0 & 0 & 1 & 1 \\
\hline G21 & 0 & l & 0 & 1 & l & 1 \\
\hline $\mathrm{G} 22 \mathrm{a}$ & 0 & 0 & 0 & 1 & 1 & 0 \\
\hline G23 & 0 & 0 & 0 & 1 & 1 & 1 \\
\hline $\mathrm{G} 24 \mathrm{a}$ & 0 & 1 & 0 & 1 & 1 & 1 \\
\hline G25 & 0 & 0 & 0 & 1 & 1 & 0 \\
\hline G26 & 0 & 0 & 0 & 1 & 1 & 0 \\
\hline G27 & 0 & 0 & 0 & 1 & 1 & 1 \\
\hline G27a & 0 & 0 & 0 & 1 & 1 & 0 \\
\hline $\mathrm{G} 27 \mathrm{~b}$ & 0 & 0 & 0 & 1 & 1 & 0 \\
\hline G30 & 0 & 0 & 1 & 1 & 1 & 1 \\
\hline G32 & 0 & 0 & 1 & 1 & 1 & 1 \\
\hline G34 & 0 & 0 & 1 & 1 & 1 & 0 \\
\hline G34a & $\mathrm{N}$ & 2 & 1 & 1 & 1 & $\mathrm{~N}$ \\
\hline G34b & 0 & 2 & 2 & 2 & 2 & 1 \\
\hline G34c: & 0 & 1 & 0 & 1 & 1 & 1 \\
\hline G34d & 0 & 0 & 0 & 1 & 1 & 0 \\
\hline G35a & 0 & 1 & 1 & 1 & 1 & 0 \\
\hline G36a & 0 & 2 & 0 & 0 & 1 & 2 \\
\hline G37 & 0 & 1 & 1 & 3 & 3 & 2 \\
\hline Lla & 0 & 0 & 0 & 1 & 1 & 0 \\
\hline L4 & 0 & 0 & 0 & 0 & 1 & 1 \\
\hline 1.6a & 0 & 0 & 0 & 1 & l & 0 \\
\hline L7 & 0 & 1 & 0 & 2 & 2 & 3 \\
\hline L8 & 0 & 0 & 0 & 1 & 1 & 0 \\
\hline L9 & 0 & 0 & 0 & 1 & 1 & 1 \\
\hline L10 & 0 & 0 & 1 & 1 & 1 & 1 \\
\hline $\mathrm{L} 13$ & 0 & 0 & 1 & 1 & 1 & 1 \\
\hline L15 & $\mathrm{N}$ & 1 & 2 & 2 & 2 & 2 \\
\hline L $15 a$ & $N$ & 1 & 2 & 2 & 2 & 2 \\
\hline $\mathrm{L} 15 \mathrm{~b}$ & $\mathrm{~N}$ & 1 & 2 & 2 & 2 & 2 \\
\hline Ll6a & 0 & 1 & 1 & 1 & 1 & 0 \\
\hline L17 & 0 & 0 & $\mathrm{~N}$ & 1 & 1 & 0 \\
\hline
\end{tabular}


Table 1 contd.

\begin{tabular}{|c|c|c|c|c|c|c|}
\hline & Ancestor & Lepidosauria & Chelonia & Crocodylia & Aves & Mammalia \\
\hline L18 & 0 & 0 & 1 & 1 & 1 & 1 \\
\hline L19 & 0 & 0 & 1 & 1 & 1 & 1 \\
\hline A6 & 0 & 1 & 1 & 1 & 1 & 0 \\
\hline A7 & 0 & 0 & 0 & 1 & 1 & 1 \\
\hline $\mathrm{A} 8$ & 0 & 0 & 0 & 1 & 1 & 0 \\
\hline A9 & 0 & 1 & 0 & 1 & 1 & 0 \\
\hline A 10 & 0 & 0 & 0 & 1 & 1 & 0 \\
\hline All & 0 & 1 & 1 & 0 & 1 & 0 \\
\hline $\mathrm{Al} 2$ & 0 & 0 & 0 & 1 & 1 & 1 \\
\hline $\mathrm{A} 13$ & 0 & 0 & 0 & 0 & 1 & 1 \\
\hline $\mathrm{Al} 4$ & 0 & 1 & 0 & 1 & 0 & 0 \\
\hline A15 & 0 & 1 & 1 & 2 & 2 & 0 \\
\hline A 16 & 0 & 0 & 0 & 1 & 1 & 0 \\
\hline A 17 & 0 & 1 & 1 & 1 & 1 & 0 \\
\hline A 18 & 0 & 0 & 0 & I & 1 & 0 \\
\hline Al9 & 0 & 1 & 1 & 1 & 1 & 0 \\
\hline $\mathrm{A} 20$ & 0 & 1 & 0 & 1 & 1 & 0 \\
\hline A21 & 0 & 0 & 0 & 1 & 1 & 1 \\
\hline A22 & 0 & 0 & 0 & 1 & 1 & 0 \\
\hline $\mathrm{A} 23$ & 0 & 1 & 1 & 1 & $\mathrm{~N}$ & 0 \\
\hline A 24 & $\mathbf{N}$ & 2 & 1 & 1 & 1 & 2 \\
\hline A25 & 0 & 1 & 0 & 1 & 1 & 0 \\
\hline A 26 & 0 & 0 & 0 & 0 & 1 & 1 \\
\hline $\mathrm{A} 27$ & 0 & 0 & 0 & 1 & 1 & 0 \\
\hline A 28 & 0 & 0 & 0 & 1 & 1 & 0 \\
\hline A 29 & 0 & 1 & 1 & 1 & l & 0 \\
\hline A 30 & 0 & 0 & 0 & 1 & l & $\mathrm{N}$ \\
\hline A31 & 0 & 0 & 0 & 1 & 1 & 1 \\
\hline A 32 & 0 & 0 & 1 & 1 & 0 & 1 \\
\hline $\mathrm{A} 33$ & 0 & 0 & 0 & 1 & 1 & 1 \\
\hline A 34 & 0 & 0 & 0 & 1 & 0 & 1 \\
\hline A35 & 0 & 1 & 1 & 1 & 0 & 0 \\
\hline A 36 & 0 & 0 & 0 & 1 & 1 & I \\
\hline A 37 & 0 & 0 & 1 & 0 & 0 & 1 \\
\hline A38 & 0 & 0 & 1 & 0 & 1 & 1 \\
\hline A 39 & 0 & 0 & 0 & 1 & 0 & 1 \\
\hline $\mathrm{A} 40$ & 0 & 1 & 0 & 1 & 0 & 1 \\
\hline A4 1 & 0 & 0 & 1 & 0 & 1 & 0 \\
\hline $\mathrm{A} 42$ & 0 & 1 & 1 & 1 & 0 & 1 \\
\hline $\mathrm{A} 43$ & 0 & 0 & 0 & 0 & 1 & 1 \\
\hline A 44 & 0 & 0 & 0 & 1 & 1 & 0 \\
\hline $\mathrm{A} 45$ & 0 & 0 & 0 & 1 & 0 & 1 \\
\hline A 46 & 0 & 1 & 0 & 1 & 1 & 0 \\
\hline A 47 & 0 & 1 & 0 & 0 & 1 & 0 \\
\hline A 48 & 0 & 0 & 0 & I & 1 & 0 \\
\hline A49 & 0 & 0 & I & 1 & 1 & 1 \\
\hline $\mathrm{A} 50$ & 0 & 0 & 0 & I & 1 & 0 \\
\hline A 51 & 0 & 0 & 0 & 0 & 1 & 1 \\
\hline $\mathrm{A} 52$ & 0 & 1 & 0 & 1 & 1 & 1 \\
\hline $\mathrm{A} 53$ & 0 & 0 & 0 & l & 1 & 0 \\
\hline
\end{tabular}

are unlike other amniotes in that "cell cords of the pars distalis grow dorsoventrally" rather than anteroposteriorly. Thus, we infer that crocodiles and birds are more like one another in the histology and development of the pituitary than either is to mammals or other amniotes. This character is referred to as $\mathrm{Lla}$ in our reanalysis (Table $\mathrm{l}$ ).

(The dorsoventral grow th of the pars distalis is diagnostic of Archosauria on both the Recent tree and our hypothesis (Figs 2,3).) 


\section{Pars Tuberalis (Only Monotremes)}

Lovtrup (table 1: 19) listed a pars tuberalis of the adenohypophysis as an apomorphy shared by only birds and mammals (at least monotremes), again citing Wingstrand (1951). Elsewhere, Løvtrup (p. 466) seems to have contradicted himself by implying that the pars tuberalis is absent only in squamates among amniotes. Indeed, Wingstrand (p. 130) stated explicitly that the "lobi laterales give rise to a pars tuberalis in birds, crocodiles, chelonians and Rhynchocephalia." Thus, we infer that the condition in birds and monotremes represents the ancestral condition in amniotes, and the absence in squamates is secondary (see section "Data and Methods of Analysis"). We have eliminated this character from our reanalysis.

\section{Post-ganglionic Nerves from Segmental Ganglia Through Plexuses and Ganglia}

Apparently, Lovtrup (table 1: 20) was referring to the following passage in Carter (1967:350): "Postganglionic nerves pass from the segmental ganglia to the organs in the body cavity and elsewhere. In the birds and mammals but not in lower vertebrates, the postganglionic nerves to the body cavity often pass through plexuses and ganglia among the organs. In these ganglia the neurons are relayed." Such a conclusion of synapomorphic resemblances may be premature. A more recent review of the autonomic nervous system in turtles, lepidosaurs and crocodiles (Berger and Burnstock, 1979), emphasized just how poorly known these taxa are compared to mammals and birds. In fact, Berger and Burnstock (p. 46) concluded that in the "sympathetic nervous system, the distribution and physiological actions of the abdominal pathways, and of the cranial and pelvic extensions of the system, have barely been investigated." In our opinion, there is at present too little information available from which to conclude the level of generality of this character.

\section{Sino-aurigular and Auriguloventricular Nodes}

According to Baumel (1979: 350), there is a consensus among modern workers "that a discrete sinuatrial node is present in the avian heart." The sino-auricular (sinu-atrial) node is the "pacemaker" initiating heartbeat, and it is also present in mammals (Lovtrup, table 1: 21). The sino-auricular node appears to derive from a portion of the sinus venosus, which acts as the pacemaker in other tetrapods (Carter, 1967). We believe the evolution of the node is not entirely independent of Gardiner's character 21, "loss of sinus venosus...," and therefore have not recorded it in our reanalysis.

We accept Løvtrup's (table 1:21) conclusion that birds and mammals are alone among tetrapods in having auriculoventricular nodes. In addition, birds and mammals possess bundles of branched striated muscle fibers modified for rapid conduction, usually called Purkinje fibers. When the pacemaker's impulse reaches the auriculoventricular node, the wave of excitation spreads along Purkinje conducting myofibers assuring coordinated contraction of the ventricles (Carter, 1967; Baumel, 1979). Berger and Burnstock (1979) noted that histologically and ultrastructurally distinct fibers are present in the region of the auriculoventricular junction of other amniotes. These fibers could represent a precursor of the auriculoventricular node and Purkinje fiber systems of mammals and birds. We have used this character in our reanalysis (Table 1: L4).

(The evolution of an auriculoventricular node and Purkinje fibers is ambiguous on the 
Recent tree (Fig. 2), where they either arose in the ancestors of thecodonts and reversed in crocodiles, or they evolved independently in birds and mammals. Our hypothesis (Fig. 3) is consistent only with the latter interpretation.)

\section{Dermal Bones Below the Skin}

We have been unable to comprehend what Lovtrup (table 1:22) meant by stating that birds and mammals are alone among tetrapods in having dermal bones below the skin. Although Løvtrup cited Carter (1967) as the source for this character, Carter makes it clear that the mere possession of dermal bone below the skin applies at a far more general taxonomic level. We have discarded this character.

\section{JACOBSON'S OrGAN}

Løvtrup (table 2: 11) considered the reduction or absence of Jacobson's organ a synapomorphy of crocodiles, birds, and mammals, even though the organ is welldeveloped in mammals. Gardiner also considered several characters of the nose in his analysis of amniote phylogeny (see nos 8 and 24 above), and he accepted Rosen et al.'s (1981) conclusion that the vomeronasal organ is a synapomorphy uniting dipnoans and tetrapods (p. 216). Considerable controversy surrounds the nature of that organ and we take this opportunity to review the problem.

Rosen et al. (1981: 201-202) provided a brief argument for the presence of a large vomeronasal organ and rudiments of a vomeronasal nerve and accessory olfactory bulb in dipnoans. 'Their argument was predicated on the gross similarity and topographic relations of a lateral diverticulum of the nasal sac in dipnoans and lissamphibians, and their interpretation of Rudebeck's (1945) claim that rudiments of an accessory olfactory bulb appear in the ontogeny of the forebrain of the dipnoan Protopterus.

It is curious that the vomeronasal nerve and accessory bulb could at best be described as only "rudimentary" when the dipnoan lateral diverticulum said to be homologous with the vomeronasal organ is so large and richly supplied with sensory epithelium. Indeed, Rosen et al. (1981) may have been a little overzealous in their inference that the vomeronasal organ is present in dipnoans, as can be seen in the following quotation from Rudebeck (1945: 95):

\footnotetext{
"Notwithstanding the fact that it has not been possible 10 identify an organ vomeronasale or a distinct vomeronasal nerve, the conditions in the anterior part of the forcbrain do not wholly speak against the occurrence of a rudimentary accessory olfactory bulb in Protopterus."
}

Upon further examination of the literature on the ontogeny of the lateral diverticulum in dipnoans and the vomeronasal organ of tetrapods, Rudebeck's inability to identify the former as the homologue of the latter becomes clear. Most other workers have objected to this putative homology for the simple reason that the ontogenetic origin and subsequent innervation of these structures are entirely different. In dipnoans, the lateral diverticulum arises as an evagination at the posterodorsal end of the lateral wall of the main nasal cavity, and it is innervated by an external (dorsal or lateral) trunk of the olfactory nerve (Fullarton, 1933; Rudebeck, 1945; Bertmar 1965, 1966). In tetrapods, however, the vomeronasal organ arises at the anteroventral end of the medial wall of the nasal cavity and is innervated by an internal (= ventral or medial) trunk of the olfactory nerve (Seydel, 1895; Parsons, 1959). Moreover, the more ventral or lateral position of the vomeronasal organ in living lissamphibians results from rotation of the wall of the 
nasal cavity during ontogeny, concomitant with the straightening of the flexed cranium of the embryo, which carries the organ from the originally anteroventromedial position ancestral for amphibians (Schmaulhausen, 1968: 167) and retained in embryonic (or paedomorphic) lissamphibians (Jurgens, 1971), to the more ventrolateral condition diagnostic of adult lissamphibians (Sarasin and Sarasin, 1890; Seydel, 1895; Hinsburg, 1901; Foske, 1934; Matthes, 1934; Schuch, 1934; Jurgens, 1971; Badenhorst, 1978). There remains the possibility that one of the medial diverticulae reported in dipnoans (Rudebeck, 1945; Bertmar, 1965) might represent a structure broadly homologous with the definitive vomeronasal organ of tetrapods. However, Northcutt's (pers. comm.; 1986) more recent work on dipnoans indicates that there is as yet no evidence that they possess either a vomeronasal organ or the characteristic neural specializations, the vomeronasal nerve and accessory bulb, associated with it.

$\Lambda$ definitive vomeronasal organ, the so-called unterer Blindsack of earlier authors (e.g., Born, 1876; Seydel, 1895), is present in all three groups of lissamphibians (Jurgens, 1971; Badenhorst, 1978) and at least some amniotes (Parsons, 1959; and see below). In its definitive adult form, the vomeronasal organ can be distinguished from unmodified olfactory epithelium by at least two criteria, one based on the histology and topographic relations of the tissue itself, and another relating to neural linkages receiving the sensory input from this tissue: (1) vomeronasal tissue is devoid of Bowman's glands and lies ventral or ventromedial to the more dorsally placed olfactory epithelium; (2) vomeronasal tissuc is innervated by a distinct vomeronasal nerve that terminates in an equally distinct accessory olfactory bulb, which in turn projects to a cell group in the anterior telencephalon called the pars lateralis of the amygdula in lissamphibians, the nucleus corticalis in mammals, and the nucleus sphericus in lepidosaurs (Northcutt, 1981). By these criteria, a fully developed vomeronasal organ is found only in lissamphibians, mammals, and lepidosaurs among Recent tetrapods. As will be discussed below, however, ontogeny and certain details of adult morphology complicate this picture.

Various authors have doubted the proposed homology of this structure, both within lissamphibians and between lissamphibians and amniotes (e.g., Mikhalkovics, 1898; Zuckerkandl, 1910; Francis, 1934). Parsons (1959) noted that their reasons for doing so were inadequate, based as they were on the assumption that "difference" necessitates the conclusion of non-homology. Parsons (1959) reviewed some of the objections, which fell into two main categories, one pertaining to morphological relations of the putative vomeronasal organ itself, and another to its neural connections. The objections may be summarized as follows. (1) The structures in question differ in gross morphology and topographic relations in adults, and the distribution of sensory epithelium within them also differs. (2) There are differences between amniotes and lissamphibians regarding the relative positions of the accessory and main olfactory bulbs and the positions at which vomeronasal nerve fibers enter the former.

As Parsons (1959) noted, although the observations supporting (1) and (2) are accurate, they lose much of their force in view of the similar ontogenetic origin of this structure in all tetrapods; regardless of its adult structure and relations (or lack thereof), the vomeronasal organ arises as a small groove in thickened epithelium at the anteroventral margin of the medial wall of the nasal cavity in the embryo (see below). Moreover, the distribution of sensory epithelium also varies within the amniote vomeronasal organ (Parsons, 1959).

$\Lambda$ third objection was raised by Zuckerkandl (1910), who noted that the position of the accessory olfactory bulb in lissamphibians varies in the dorsoventral plane, but that 
it was nonetheless posterolateral to the main olfactory bulb, and that vomeronasal fibers enter it anteriorly or laterally, but not medially. Zuckerkandl argued further that in amniotes the accessory bulb lies posteromedial to the main olfactory bulb, with many of the vomeronasal nerve fibers entering medially. Parsons (1959) pointed out that the position of the olfactory bulb was equally variable in amniotes, and cited Crosby and Humphrey (1939a,b) as noting a dorsolateral position of the accessory bulb in at least some mammals. McCotter (1917) showed that the vomeronasal nerve enters the accessory bulb anteriorly in turtles, suggesting that the entrance of the vomeronasal nerve varies within amniotes as well. Thus, as Parsons (1959) argued, neither of Zuckerkandl's objections to the homology of these structures across tetrapods can be considered compelling.

Considerable controversy surrounds the question of the taxonomic level of origin of Jacobson's organ, a tubular or spherical structure containing the vomeronasal organ. Following Parsons (1959, 1967, 1970), Gaffney (1980) considered Jacobson's organ diagnostic of a taxon composed of amniotes exclusive of turtles. In the view of these authors, turtles are considered to have retained the ancestral vomeronasal organ. However, for reasons that will become apparent below, this does not seem to be the case.

The vomeronasal organ lies in a diverticulum of the nasal cavity in adult lissamphibians, mammals, and lepidosaurs. This diverticulum is absent in adult birds, crocodiles, and turtles, although turtles typically possess as many as three broad and shallow sulci covered by vomeronasal epithelium (Parsons, 1959). Moreover, turtles are like other tetrapods (except crocodiles and birds) in possessing a distinct vomeronasal nerve and accessory olfactory bulb, and thus a functional vomeronasal organ (McCotter, 1917). Indeed, turtles differ from all other tetrapods in that the vomeronasal epithelium lies on the floor of the intermediate region of the cavum nasi proprium, rather than being contained in a diverticulum of the nasal cavity in the adult (Seydel, 1896). Further, a thickened area of sensory epithelium, that subsequently becomes innervated by the vomeronasal nerve, first appears along the ventromedial wall of the nasal cavity in all tetrapods, including turtles (Seydel, 1895, 1896). Evidently, the lateral spread across the floor of the nasal cavity takes place only in the later stages of turtle ontogeny (Seydel, 1896; Nick, 1912; Fuchs, 1915). Thus, the initial condition of epithelial thickening at the anteroventral midline is present in all tetrapods, but not in their outgroups among vertebrates. Within tetrapods, there are two subsequent transformations; one is the evagination and formation of a diverticulum in lissamphibians and amniotes aside from turtles, and the other is the lateral spread of this tissue that is seen only in turtles. But which condition is ancestral? Parsons and Gaffney have accepted the hypothesis that the diverticulae of lissamphibians and non-chelonian amniotes are not homologous, thus treating the lateral spread of the vomeronasal epithelium as an ancestral condition retained in turtles. In their view, there are two evolutionary events, an appearance of a diverticulum in lissamphibians and one in non-chelonian amniotes. $\Lambda$ s noted by Parsons (1959), however, there is an equally parsimonious alternative interpretation; one appearance of the diverticulum in the ancestral tetrapod and its subsequent transformation into an apomorphic condition diagnostic of turtles. Both scenarios require the same number of evolutionary changes.

This still leaves the problem of Jacobson's organ itself, the structure that is present in adult mammals and lepidosaurs alone among amniotes, which brings us to a consideration of Parsons' (1959) definition of this organ. According to Parsons (1959), it seems that to qualify for the name Jacobson's organ, the organ must appear initially as 
an inpocketing of the nasal pit prior to the time at which fusion of the lateral and medial nasal processes separates the external naris from the choana. All are agreed that such an event takes place in the ontogeny of mammals and lepidosaurs, but there has been a variety of opinions as to the presence or absence of this event in turtles, crocodiles, and birds. This is not surprising, because even those who argue for its presence in these groups claim no more than a fleeting appearance during ontogeny, and are agreed that no such inpocketing persists in any of these taxa after fusion of the medial and lateral nasal processes (e.g., Parsons, 1959). In Parsons' 1959 and subsequent reviews, he accepted the presence of this event in crocodiles and birds, but denied that it takes place in turtles. This may be splitting hairs, however, because both Seydel (1896) and Fuchs (1907) figured a slight curve in the nasal pit in turtles; Parsons maintained that this "slight curve" differs fundamentally from the "distinct groove" of other amniotes, although in the single Alligator in which he found such a structure, it was barely more conspicuous than in his figure of Chrysemys (compare figs. 11B and 69B in Parsons, 1959, and fig. 1 in Grewe, 1951, for the condition in an embryonic bird). Although there is a considerable literature on the ontogeny of the nasal region of crocodiles, Parsons' assertion that this inpocketing occurs in this group appears to have been based on only two specimens, on Crocodylus niloticus figured in Voeltzkow (1899) and one among the thirty-one embryos of Alligator studied by Parsons (1959). Likewise, this infolding appears to have been identified in only three or four bird embryos. It is not that we doubt the accuracy of these observations; rather, the dearth of unambiguous observations indicates that it is difficult to find this fleeting developmental event. Thus, although we score the event of "infolding" as absent in turtles, further research on turtle development may demonstrate its presence.

Lissamphibians differ from amniotes in that evagination (= inpocketing) begins after, rather than before, the fusion of the medial and lateral nasal processes (Parsons, 1959). Further, lissamphibians are unlike amniotes in that the small groove-like pit that originates in thickened epithelium continues to elongate during the ontogeny of lissamphibians, so that the vomeronasal and main nasal chambers are in broader communication than is the case in adult amniotes (this communication becomes further modified in adult caecilians; Badenhorst, 1978). In the absence of any such transformation in an outgroup, however, it is not possible to specify which of the two transformations found among tetrapods represents the ancestral condition.

Based on our review of the literature on the vomeronasal organ, the following conclusions appear to be in order. (1) A vomeronasal organ was present in the ancestral tetrapod, but it was absent in the common ancestor of choanates. (2) If Schmaulhausen (1968) was correct about the position of the vomeronasal organ in extinct "amphibians", then the lateral rotation of the organ during ontogeny would be diagnostic of lissamphibians, and amniotes would retain the ancestral tetrapod position of the vomeronasal organ. (3) If turtles are, as Parsons and Gaffney suggest, the sister group of other amniotes, then it cannot be determined if lateral spread or medial invagination represents the ancestral condition. However, if mammals are the sister group of all other amniotes (Fig. 3), then medial evagination was the ancestral condition, and turtles would be diagnosable by lateral spreading of vomeronasal tissue. (4) Archosaurs (birds and crocodiles) are diagnosed among amniotes by the absence of all components of the vomeronasal system, with the possible exception of the temporary appearance of a slit-like evagination in the embryo (Table 1: L6a). (5) The criteria heretofore employed to distinguish Jacobson's organ as a transformation of the 
vomeronasal organ within amniotes cannot be polarized. Accordingly, current knowledge dictates that this structure should be referred to as the vomeronasal (or Jacobson's) organ in all tetrapods, rather than as a vomeronasal organ in lissamphibians and turtles, and a Jacobson's organ in the remaining amniotes.

(The absence of a vomeronasal organ in adults diagnoses $\Lambda$ rchosauria on both trees (Figs 2, 3).)

\section{Histology of Adrenal Tissue}

Løvtrup (table 2: 12) offered no citation to support his contention that the histology of the adrenal gland of crocodiles displayed apomorphic resemblances to those of mammals and birds. On the contrary, based on anatomical relations between adrenal tissue and inter-renal cords, Gabe (1970) recognized four "structural types" of adrenal glands among amniotes: a "generalized type" found in turtles; a second in lepidosaurs; a third in crocodiles and birds; and a fourth in mammals. In that they retain attributes common among lissamphibians, turtles appear to be less modified than are other amniotes, and they display a number of other ancestral conditions in adrenal morphology and histology. The transformational relationships among the remaining three types are obscure (Gabe, 1970). These data are accordingly entered in our matrix as unordered (Table 1: L7).

Løvtrup cited Gabe (1970) as the source of his conclusion that a retroperitoneal position of the adrenals is peculiar to mammals, turtles, crocodiles, and birds among amniotes. However, Løvtrup appears to have misinterpreted Gabe on this point, in that the adrenals and kidneys lie outside the peritoneum in all gnathostomes. Although Lovtrup considered lepidosaurs to be ancestral in this respect, they are in fact derived. The adrenals lie with the kidneys next to the body wall in all tetrapods except lepidosaurs, where the adrenals are suspended within the gonadal mesentery (Osawa, 1897; Gabe, 1970); in either case, the gland is uniformly retroperitoneal in position.

(Several evolutionary histories are possible, because of the unordered aspects of adrenal gland morphology. The only unambiguous evidence is that the lepidosaur adrenal is autapomorphic on the Recent tree (Fig. 2), and that the mammalian adrenal is autapomorphic on ours (Fig. 3). The evolution of adrenal gland morphology will remain ambiguous until such a time as one is able to point to some hierarchical connections among the currently recognized "structural types." Given our hypothesis (Fig. 3) and the available evidence, one cannot be sure if the condition in either archosaurs, lepidosaurs, or turtles represents the ancestral condition for Sauria. Ambiguity also applies to the ancestral condition of Thecodontia on the Recent tree (Fig. 2).)

\section{Morphology of Thymus}

Contrary to Lovtrup (table 2: 13), we found no particular apomorphic similarities in thymus morphology shared by crocodiles, birds, and mammals. Bockman (1970: 115), cited by Lovtrup, actually stated that crocodiles "have a thymus more like that of birds than that typical of other reptilian groups." As in amniotes generally, there are at least two pairs of such organs in crocodiles and birds, and they share no particular similarity with the single bilobed organ in mammals (Bentley, 1976). Thus, we have limited this apomorphy to crocodiles and birds. 
(The crocodile bird type of thymus morphology evolved in the ancestors of archosaurs according to both trees (Figs 2, 3).)

\section{Three Neurofilament Proteins}

Løvtrup (table 2: 14), citing the work of Shaw et al. (1984) and G. Shaw (unpublished) on amniote neurofilament proteins, considered the possession of three neurofilament proteins diagnostic of crocodiles, birds, and mammals. Shaw et al. (1984) noted that two neurofilament polypeptides, of lower and middle molecular weights, are present in lissamphibians and teleosts; both are also present in amniotes, but mammals, crocodiles, and birds appear further derived in possessing a high molecular weight polypeptide. The middle polypeptide of turtles and lepidosaurs displays cross-reactivity with both mid-and higher weight polypeptides of other amniotes. Without comparing the middle weight polypeptide of lissamphibians to those of other amniotes, it is difficult to assess homologies, but we tentatively accept Løvtrup's interpretation of polarity among these states (Table 1: L9).

(The possession of three neurofilament polypeptides diagnoses Thecodontia on the Recent tree; however, our hypothesis indicates that it evolved independently, once in mammals, and once in the ancestor of Archosauria.)

\section{Cartilage Cells in Epiphysis}

Løvtrup's reference to cartilage cells (table 3: 10) appears to be a lapsus calami. We believe Løvtrup meant to refer to cartilage canals, because he cited Haines (1969), who noted the presence of these structures in the epiphyses of mammals, turtles, crocodiles, and birds, but not lepidosaurs (except Varanus). Cartilage canals are not present in amphibian epiphyses (Haines, 1942), so lepidosaurs appear to have retained the ancestral condition (Table 1: L10).

(The presence of cartilage canals in epiphyses diagnoses Euamniota on the Recent tree (Fig. 2). In our hypothesis (Fig. 3), their presence diagnoses Amniota, with a reversal in lepidosaurs.)

\section{Vascular Bone}

In contrast to other authors, Løvtrup (table 3: 11) thought that the avascular periosteal bone of lepidosaurs represented the ancestral condition for amniotes. However, periosteal bone is vascular in lissamphibians, mammals, turtles, crocodiles, and birds, which is why Enlow (1969) considered the predominance of avascular bone diagnostic of lepidosaurs. Given only this autapomorphy, we have omitted the character from our reanalysis.

\section{Nonlobed Thymus}

The thymus is not lobed in lepidosaurs, and Lovtrup (table 3:12) considered a lobulate mammal, turtle, crocodile, and bird condition derived. Løvtrup failed, however, to consult the outgroup for polarity determination. Cooper (1976) described the lissamphibian thymus as lobulate; hence, the condition seen in lepidosaurs is diagnostic, and not an ancestral condition as Løvtrup suggested. This autapomorphy has been excluded from our data matrix. The paired, lobulate thymus of crocodiles and birds has been discussed above (character no. 8 this section). 


\section{Sinus Cavernosus}

Løvtrup (table 3: 13), citing Wingstrand (1951), considered the presence of "sinus cavernosus", large venous caverns in the sella, to be diagnostic of mammals, turtles, crocodiles, and birds. However, Wingstrand (p. 268) actually stated that the "term is used because it describes the structure well--there is in fact a cavernous sinus - and it is not regarded as a comparative anatomical term. In fact, the sinus cavernosus of birds is not homologous with that of mammals." Wingstrand's conclusion of non-homology is derived from his own work on birds, and from ontogenetic investigations of the region in non-avian amniotes by Shindo (1915). Both authors rejected homology on the basis of developmental differences. Wingstrand pointed out, however, that a "sinus cavernosus" is considered homologous throughout mammals even though van Gelderen $(1924,1925)$ noted developmental differences; a continuous development of the vena capitis medialis into a "sinus cavernosus" is seen only in monotremes and primates, whereas in other mammals, the sinus arises secondarily after the vein has disappeared. Given that this is the case, we cannot accept Wingstrand's conclusion of non-homology across amniotes merely because there are differences in development. There are at least some similarities (e.g., Shindo, 1915), and at this stage of analysis, we must admit that it is possible that the "sinus cavernosus" has been conserved through phylogeny, even though the developmental pathway through which it arises may have evolved (de Beer, 1958). Although lepidosaurs also have an enlarged vein passing transversely behind the pituitary, they are like lissamphibians in having no real "sinus cavernosus" (Wingstrand, 1951). The same vein in the embryos of other amniotes, the so-called "vena retrohypophysea", produces the "sinus cavernosus" in turtles, crocodiles, and birds, as well as the sinus intercavernosus posterior, which connects the two "sinus cavernosi" behind the pituitary in mammals (Shindo, 1915). One condition is shared by turtles, crocodiles, and birds, and another by mammals, but neither is present in lepidosaurs or lissamphibians. Accordingly, we have recorded the absence as ancestral, and the autapomorphy of Mammalia has been omitted (Table 1: L13). Although we conclude that the "sinus cavernosus" may be homologous, in that it is at least partly derived from the embryonic vena retrohypophysea in these groups, many other aspects of the morphology of this region cannot be polarized.

Wingstrand (1951) also discussed the distribution of an intercarotid anastomosis among tetrapods, noting that it is absent in lissamphibians and lepidosaurs (except one species of Chamaeleo). He also stated that it was absent in mammals, although Baumel and Gerchman (1968) showed that Wingstrand was mistaken in this regard. $\Lambda$ ccording to Baumel and Gerchman, an intercarotid anastomosis is also present in nontetrapods, including lungfish, as well as in turtles, crocodiles, and birds. Thus, at this stage of analysis, the evolution of this character is equivocal; the anastomosis could have been lost in tetrapods ancestrally, and subsequently re-evolved in Gardiner and Løvtrup's putative common ancestor of mammals, turtles, crocodiles, and birds; or it could have been lost independently in lissamphibians on the one hand, and in lepidosaurs on the other. We agree with Wingstrand that the latter set of transformations is more likelyparticularly because carotid circulation is exceptional in lissamphibians - but this interpretation yields autapomorphies for lissamphibians and lepidosaurs, which are not considered in this analysis. Therefore, this character has not been included in our data matrix (see "Data and Methods of Analysis").

(The specialized sinus cavernosus diagnoses Euamniota on the Recent tree (Fig. 2), 
whereas our hypothesis (Fig. 3) suggests that it characterizes Amniota and has reversed in Lepidosauria.)

\section{Similarities in Egg Shell.}

Lovtrup (table 3: 15) cited only Hill's (1933) research on monotremes, and it is difficult to be sure which similarities in the egg shells of mammals, turtles, crocodiles, and birds he considered synapomorphic. Løvtrup did use the presence of a "limeimpregnated shell' (p. 466) as evidence that Sphenodon shares a more recent common ancestor with this group than it does with Squamata. However, the tuatara, like the vast majority of squamates, exhibits a parchment-like eggshell which contains relatively little calcareous matter (Dendy, 1899; Dawbin, 1982). Sea turtles and some emydids have a parchment egg shell; however, we interpret these as secondarily derived (Pritchard, 1979). The presence of a calcareous shell is included in our reanalysis (Table 1: L15). The ingroup variants do not exist in the outgroups, and an $\mathrm{N}$ is attributed to the Ancestor in the data matrix.

Additional similarities in the eggs of mammals (Hill, 1933), turtles, crocodiles, and birds, which are absent in lepidosaurs, include the presence of numerous large pores in the outer covering of the egg and the presence of a pair of tertiary egg membranes (Dendy, 1899; Packard et al., 1977). It seems likely that having these features is functionally correlated with possessing a calcareous shell. However, we have included these characters in Table 1 ( $\mathrm{L} 15 \mathrm{a}, \mathrm{L} 15 \mathrm{~b}$ ) to avoid a bias against the Gardiner-Løvtrup classification. The homology of the tertiary membranes with the various coverings of the eggs of the outgroups is unclear.

(The phylogenetic history of a thick calcareous shell with pores and a pair of tertiary egg membranes is equivocal according to the Recent hypothesis (Fig. 2). Either lepidosaurs are relatively primitive, and the three egg states diagnose Euamniota, or those states characterize amniotes, with lepidosaurs being derived. On our tree (Fig. 3), all three characters arose in amniotes, with their absence unambiguously delimiting lepidosaurs. Our interpretation is also consistent with the morphology of eggs referrable to various groups of extinct amniotes.)

\section{Aromatic Acid Conjugation}

Løvtrup (table 3: 16) concluded from Jordan et al. (1980) that turtles, crocodiles, birds, and mammals share an apomorphic resemblance in benzoic acid conjugation. Løvtrup (p. 465) claimed that "when benzoic acid is given to reptiles and birds, ornithuric acid is excreted, but in turtles, crocodiles, and birds some is conjugated with glycine to yield hippuric acid. In mammals, only hippuric acid is formed." Lovtrup's conclusion is, however, at odds with that of Jordan et al. (1980), who stated clearly in the introduction (p. 101) that "mammels [sic], amphibia, and fish ... conjugate benzoic acid preferentially with glycine or glucuronic acid" to produce hippuric acid. Having established that the ancestral pathway leads to hippuric acid (contra Lovtrup), we will consider the production of ornithuric acid in benzoic acid metabolism as an apomorphy shared by turtles, lepidosaurs, crocodiles, and birds (Table 1: L16a). Jordan et al. (1980) also argued that the paucity of hippuric acid production in benzoic acid conjugation in lepidosaurs is a diagnostic autapomorphy, and not an ancestral condition as Løvtrup suggested.

(The production of ornithuric acid is diagnostic of Amniota, and mammals exhibit a 
reversal on the Recent tree (Fig. 2). However, according to our hypothesis (Fig. 3), mammals retain the ancestral condition and this character is a distinguishing feature of Reptilia.)

\section{Uncinate Processes on Ribs}

Løvtrup (p. 466) suggested that uncinate processes might be diagnostic of a group including Sphenodon, turtles, crocodiles, birds, and mammals. Uncinate processes are, however, absent in mammals, as they are in the outgroups. Moreover, the intimate association of the ribs with the dermal skeleton of the carapace makes this character difficult to interpret in living turtles. Thus, these neomorph cartilages are present only in some lepidosaurs (Sphenodon) but not others (squamates), and in crocodiles and birds. Uncinate process calcify during postnatal ontogeny in Sphenodon and crocodiles, but they become ossified in birds (Heilmann, 1927). Because of variation among lepidosaurs (sce "Data and Methods of Analysis"), only birds and crocodiles are listed as having the apomorphic condition (Table 1: L17).

(On the Recent tree (Fig. 2), uncinate processes are diagnostic of archosaurs. This character is not listed in Table 2 because ingroup hypotheses of relationship involving fossils indicate that it appeared within sphenodontid lepidosaurs (Gauthier et al., 1988a) and within coelurosaur theropods (Gauthier, 1986).)

\section{Albumen}

Lovtrup (p. 466) claimed that Sphenodon is like mammals, turtles, crocodiles, and birds in having egg albumen (see also Løvtrup, 1977). The distinction that he seems to have been making regarding this protein is the amount present: large amounts in mammals, turtles, crocodiles, and birds, and relatively little in squamates, at least at the time of laying (Badham, 1971). Contrary to Lovtrup, Sphenodon also has a small amount of albumen (Dendy, 1899; Packard et al., 1977). Traditionally, albumen is not mentioned as present in the eggs of the outgroups; however, there seems to be no strong argument for this exclusivity. We believe the topographic and compositional similarities of capsules and albumen are sufficiently great to propose that the relatively small jelly capsules of lissamphibians are the forerunner(s) of amniote albumen, and the ancestral and lepidosaur states are recorded as the same in terms of a mount; all other amniotes are derived (Table 1: L18).

(The presence of a relatively large amount of egg albumen diagnoses Euamniota on the Recent Tree, whereas according to our hypothesis (Fig. 3) it is an amniote character which reversed in lepidosaurs.)

\section{Horny Caruncle}

Lovtrup (p. 466) cited the presence of a horny caruncle as diagnostic of the group which includes Sphenodon, mammals, turtles, crocodiles, and birds. The caruncle is a cornified embryonic structure, formed by extra layers of epidermis (de Beer, 1949), that is only used by embryos during hatching, and which is lost soon thereafter. The caruncle is supported by a distinct ossification, the os carunculae (also lost after hatching), which in turn is supported by the prenasal process of the premaxilla. This structure is often confused with the egg tooth which, although functionally similar, is a true tooth, complete with enamel organ. Both the caruncle and egg tooth are present in monotremes and vestiges of both have been identified in embryonic and pouch-young marsupials 
(Hill and de Beer, 1949). In other amniotes, only one or the other structure is present. Løvtrup interpreted the absence of the caruncle in squamates as plesiomorphic. In keeping with our position on coding within terminal taxon variation (see "Data and Methods of Analysis"), we score lepidosaurs as primitive (Table 1: L19).

(The history of the horny caruncle is ambiguous on the Recent tree (Fig. 2). It could have arisen in amniotes ancestrally and then reversed in squamates, or the caruncle could have appeared independently in Sphenodon and Euamniota. According to our hypothesis (Fig. 3), this character is unambiguously interpreted as an amniote synapomorphy that reversed in squamates.)

\section{Additional Data}

\section{Spermatid Nugleus}

Gardiner (p. 214) considered the "unique pattern of the spermatid nucleus in birds and monotremes (Carrick and Hughes, 1978)" diagnostic of his Haemothermia, although he failed to include that character in his summary (his fig. 2). Gardiner made no specific reference to a particular aspect of the spermatid nucleus that he considered synapomorphic. This presents some problems, because avian spermatozoa have been known since the 1890's to be of "two general types, the simpler one being common to most birds [non-passerines] and showing a remarkable resemblance to that found among reptiles" (McFarlane, 1963: 92). Gardiner may have been referring to the presence of chromatin condensations that are circumferentially arranged in the spermatid nuclei of monotremes and some birds. We will not consider this character further, because there are several states present in both mammals and birds (Carrick and Hughes, 1978), and within taxon variation effectively obscures the pattern of between taxon variation.

\section{FEVER}

Gardiner (p. 214) also considered synapomorphic "the fact that both mammals and birds develop a fever on infection with bacteria (Schmidt-Nielsen, 1979)." However, Kluger's (1979) review of this phenomenon reveals that the development of fever upon bacterial infection is known in a variety of insects, crayfish, scorpions, and all vertebrates. Thus, fever represents the ancestral condition for tetrapods, and this character has been discarded.

\section{Collagens}

Gardiner (p. 214) cited Miller's (1976) observation that birds and mammals share very similar collagens. "Similarity", in terms of overall resemblance, does not in itself necessarily imply recency of common ancestry (Hennig, 1966), and Miller (1976) did not provide a compelling reason for concluding that the bird-mammal condition is apomorphic, nor that it is limited to those two taxa among choanates. Thus, we have eliminated this character.

\section{Hypoglossal Roots and Postvagal Myotomes}

Gardiner (p. 216) cited de Beer (1937) as indicating that there are "four hypoglossal roots and four metotic myotomes behind the vagus in the embryo" of crocodiles, birds, and mammals, but not in other tetrapods. We are not sure that we understand 
Gardiner's intent when he referred to the number of "hypoglossal roots and metotic myotomes in the embryo", because the number of metotic or occipital somites and ventral nerve roots varies ontogenetically. As de Beer observed (1937: 23), the "literature on this subject contains a number of descriptions of occipital somites, some of which disappear and others persist. Unless, however, a complete series of stages has been studied, and unless the prootic as well as the metotic somites have been investigated, an element of uncertainty must exist as to whether the foremost metotic somite observed is really the first." Added to the ontogenetic variation, there is also considerable variation within the outgroups, thus making it difficult to assess the ancestral condition. According to de Beer (1937), among lungfishes, there are seven or eight metotic somites in Neoceratodus, six in Protopterus, and five in Lepidosiren; counts at least as high or higher also occur in other fish-like vertebrates. De Beer (1937) reported two metotic somites in salamanders and caecilians, and one in frogs, and, accordingly, a reduced number is assumed to be diagnostic of Recent lissamphibians. Among amniotes, de Beer (1937: 32) suggested "there is the possibility that the Amniote line of vertebrate evolution was characterized by the same number of segments in the head $(9$ or 9.5 according to whether the body of the proatlas centrum fuses with the odontoid process of the 1 st vertebra or with the parachordal of the skull) ever since the fish stage." Of the nine somites contributing to the amniote skull, five or six are said to be metotic (yielding at least four occipital arches) in mammals, turtles, lepidosaurs, and birds (de Beer, 1937). We have been unable to determine the number of metotic somites in crocodiles. Gardiner may have been referring to evidence of which we are not aware, but our reading of de Beer does not seem to support his contention that the embryology of this region of the skull offers special evidence for a group composed of crocodiles, birds and mammals.

\section{Pharyngeal Tonsils}

Gardiner (p. 216) claimed that birds, crocodiles, and mammals are the only amniotes with "tonsillae pharyngeae at the entrance of the eustachian tube." He offered no citation to support this claim, and we consider this condition to be ancestral for amniotes, because tonsils have also been reported in this position in turtles and lepidosaurs (Cooper, 1973; Cooper et al., 1985).

\section{Nasal Glands}

Parsons (1959) noted that all terrestrial vertebrates possess glandular outgrowths of the nasal epithelium. Of these, the external nasal gland is distinctive in that it arises posteriorly in the vestibulum, appears earliest in ontogeny, is the largest nasal gland, and is innervated by the ramus lateralis nasi of the ethmoidal nerve (Parsons, 1970). The external nasal gland lies within the nasal capsule in lissamphibians (Mathes, 1934; Badenhorst, 1978) and mammals (Broman, 1921). In contrast, this gland assumes an apomorphic position outside the nasal capsule in turtles, lepidosaurs, crocodiles, and birds (Parsons, 1959, 1970, and references therein). This character is listed as A6 in Table 1.

(According to our hypothesis (Fig. 3), the position of the nasal gland outside the nasal capsule diagnoses Reptilia. On the Recent tree (Fig. 2), this character diagnoses Amniota, and reverses in mammals.) 


\section{Stomach}

The esophagus and duodenum lie at opposite ends of the stomach, and the lumen of the stomach is situated anterior to the pyloris in lissamphibians (Duellman and Trueb, 1986). This configuration is retained in turtles and lepidosaurs (Miller and Lagios, 1970). In contrast, the pyloris has a more anterior position in crocodiles; the duodenum arises from the stomach at a point adjacent to the esophago-gastric junction, so that the bulk of the lumen of the stomach lies posterior to the pyloris (Miller and Lagios, 1970). The morphology of the stomach varies considerably in birds and mammals, but they are nonetheless crocodile-like in that a more or less considerable portion of the lumen of the stomach lies posterior to the pyloris (Owen, 1868; Ziswiler and Farner, 1972). We record the derived state in all three taxa (Table 1: $A 7$ ).

(The posterior position of the stomach, relative to the pyloris, is characteristic of 'Thecodontia on the Recent tree (Fig. 2), whereas our hypothesis (Fig. 3) suggests that it evolved independently in mammals and archosaurs.)

\section{Gizzard}

According to Luppa (1977) and Farner (1960), crocodiles and birds may be distinguished from other amniotes because the tunica muscularis is strongly developed in the zone preceding the pyloric portion of the stomach. Luppa (1977:269) noted that "As in birds, the very muscular stomach (of crocodiles) may be referred to as a gizzard." We think it is likely that swallowing stones (gastroliths) is a behavioral attribute correlated with the presence of a gizzard (see excellent review by Neill, 1971: 13-17). A less prominent muscular tunic is present in lissamphibians (Duellman and Trueb, 1986), turtles, lepidosaurs, and mammals. This character is listed as $\Lambda 8$ in Table 1.

(The presence of a gizzard diagnoses Archosauria on both the Recent tree and ours (Figs 2, 3).)

\section{Plasma Concentrations of Urea and Uric Acid}

According to H. C. Dessauer (pers. comm.), lissamphibians, mammals, and turtles display high concentrations of urea in their blood plasma, which reflects the presence of active urea-cycle enzymes (see also Brown and Cohen, 1969). In contrast, lepidosaurs, crocodiles, and birds display no more than a low urea concentration, which results from loss or suppression of the urea-cycle. We have recorded the latter condition as the derived state in our reanalysis (Table 1: A9).

(The suppression of the urea cycle is diagnostic of Sauria on our tree (Fig. 3), whereas according to the Recent hypothesis (Fig. 2), it originated independently in lepidosaurs and in the ancestors of Archosauria.)

\section{URinary Bladder}

$\Lambda$ urinary bladder is generally present in lissamphibians (Duellman and Trueb, 1986), mammals, and turtles (Fox, 1976). According to Beuchat (1986), it has been lost independently several times among lepidosaurs (Sphenodon and most squamates have retained the ancestral state). In contrast, a urinary bladder is absent in crocodiles (Fox, 1976) and birds (although Struthio has an analogous structure; Sperber, 1960). We view the absence of a urinary bladder as an apomorphy shared by crocodiles and birds (Table $1:$ Al0). 
(The loss of a urinary bladder delimits Archosauria according to both hypotheses (Figs 2, 3).)

\section{Color Vision}

Walls (1942) noted that color vision is absent in lissamphibians (with the possible exception of some frogs), mammals ancestrally, and crocodiles. In contrast, color vision is present in turtles, lepidosaurs, and birds. Walls (1942: 518) described turtles, lepidosaurs, and birds as the "only groups in which a fovea is ever seen. The bright-light habit depends upon cone-richness, affords high visual acuity, demands good accommodation, and supports good hue-discrimination. It is only natural, then, that these phenomena are found in association." We interpret this complex as derived (Table 1: $A 11$ ).

(The evolutionary history of color vision on the Recent tree (Fig. 2) is complicated. First it evolved in the ancestors of amniotes, was lost in Thecodontia, and re-evolved again in birds. According to our hypothesis (Fig. 3), color vision diagnoses Reptilia and it was lost secondarily in the nocturnal crocodiles.)

\section{Nest Building}

It is widely known that crocodiles, birds, and mammals are the only tetrapods that commonly build nests out of vegetable material, wherein their eggs are incubated (Table 1: A12).

(This habit delimits Thecodontia on the Recent hypothesis (Fig. 2); however, we conclude that it evolved independently in mammals and archosaurs (Fig. 3).)

\section{Ependymal Cells}

The growth pattern of certain neuroblasts (Northcutt, 1984, fig. 8) provides additional evidence for Gardiner's Haemothermia. Undifferentiated ependymal cells are located in the mantle layer, next to the neurococl. During ontogeny, each cell grows a process which eventually reaches the periphery of the central nervous system. All adult craniates, except birds and mammals (Cajal, 1911; Leghissa, 1962; O'Flaherty, 1970), retain these long processes. As birds and mammals approach adulthood, the processes begin to regress, and with maturity they are lost altogether (Table 1: A13).

(The growth pattern of the ependymal cells evolved convergently in mammals and in birds on our tree (Fig. 3). Its history is ambiguous on the Recent tree, where it is diagnostic of Thecodontia and reversed in crocodiles, or appeared independently in mammals and birds.)

\section{Olfactory Bulbs}

We agree with part of Benton's (1985) assessment of the relationship of the tetrapod olfactory bulbs to the rest of the brain. He claimed (p. 108) that the bulbs of crocodiles and lepidosaurs are linked to the forebrain by stalk-like olfactory tracts or peduncles (Table 1: 114). He presumed this condition was derived from the apedunculate state exhibited by lissamphibians; turtles, birds, and mammals retain the ancestral condition as well. It can be added that a few squamates also lack stalks, but these are most simply interpreted as evolutionary reversals, because Sphenodon and most other squamates possess olfactory peduncles. 
(Pedunculate olfactory bulbs evolved convergently in lepidosaurs and crocodiles on the Recent tree (Fig. 2). Our hypothesis (Fig. 3) indicates instead that the apomorphic condition diagnoses Sauria, and is reversed in birds, but only if extinct ornithosuchians are considered. Endocasts from extinct ornithosuchians retaining saurian peduncles (e.g., Hopson, 1979) demonstrated that the apedunculate condition of extant birds is secondary. Without the additional knowledge afforded by the fossil record, it would be equally parsimonious to invoke convergence as an explanation for the peduncles in extant lepidosaurs and crocodiles.)

\section{Dorsoventricular Ridge of Telencephalon}

In contrast to lissamphibians and mammals, a dorsoventricular ridge (DVR) of the telencephalon is present in turtles, lepidosaurs, crocodiles, and birds (Northcutt, 1981). Moreover, the DVR reaches its greatest degree of enlargement and elaboration in birds and crocodiles (Ulinski, 1983). Clark and Ulinksi (1984) also noted that the distribution of neurons in the ADVR (anterior dorsoventricular ridge) reveals that turtles and lepidosaurs possess a zone of cell clusters near the ventricular surface, whereas these cell clusters are more evenly distributed in crocodiles and birds. We record this variation as three states in Table 1: $\Lambda 15$ ).

(The history of the dorsoventricular ridge is equivocal according to the Recent thesis (Fig. 2), where there is a reversal either at the level of Thecodontia, or in mammals, with archosaurs continuing to possess the most elaborate DVR. However, in the context of our hypothesis (Fig. 3) the evolution of the DVR diagnoses Reptilia, with both the increasing enlargement and elaboration of the DVR, and the apomorphic distribution of neurons in the ADVR, being diagnostic of Archosauria.)

\section{RhOMBENGEPHALON}

According to Schwab (1979), crocodiles and birds have the most differentiated rhombencephalon among amniotes, aside from mammals. Northcutt (pers. comm.) pointed out that rhombencephalic differentiation occurs mainly in areas associated with hearing, and that mammals, birds, and crocodiles are the most vocal amniotes. Although these three taxa share elaborate auditory portions of the rhombencephalon, Northcutt cautioned that the specializations involve different parts of this area in mammals on the one hand, and in crocodiles and birds on the other. Schwab (1979) reported that crocodiles and birds have highly differentiated cochlear nuclei, and welldeveloped angular and magnocellular nuclei in the rhombencephalon. Schwab (1979: 195) noted further that there is "no apparent region of mammalian cochlear nuclei which receive afferents from the macula lagenae." We consider the apomorphic condition to be present only in birds and crocodiles (Table 1: Al6).

(Rhombencephalon specializations involving several nuclei are characteristic of Archosauria on both trees (Figs 2, 3).)

\section{IRIS}

Walls (1942) noted that the intraocular muscles comprising the iris and ciliary muscles in lissamphibians and mammals are smooth, whereas those of turtles, lepidosaurs, crocodiles, and birds are composed of striated muscle. Accordingly, we have recorded the latter state as derived (Table 1: A17). 
(On our tree (Fig. 3), striated iris and ciliary muscles diagnose Reptilia, whereas the Recent hypothesis (Fig. 2) suggests that this is an amniote character that reversed in mammals.)

\section{Origin of M. PIFI Pars Dorsalis (2)}

Rowe (1986b) pointed out that a portion of $M$. puboischiofemoralis internus has a dorsal origin in crocodiles and birds (pars dorsalis), rather than the exclusively ventral origin on the puboischial plate observed in other tetrapods (Table 1: A18).

(The dorsal origin of this muscle characterizes Archosauria on both trees (Figs 2,3).)

\section{Masticatory Muscle Plate}

According to Edgeworth (1935), turtles, lepidosaurs, crocodiles, and birds are alone among tetrapods in having the masticatory muscle plate divided into a constrictor dorsalis and an adductor mandibulae (Table 1: $\Lambda 19$ ).

(The division of the masticatory muscle plate is peculiar to Reptilia on our tree (Fig. 3 ); however, the Recent hypothesis (Fig. 2) suggests that it evolved in the lineage leading to Amniota, and was lost secondarily in mammals.)

\section{Position of Kidney}

According to Gabe (1970), the kidney is closely appressed to the adrenal gland in anamniote vertebrates. Among amniotes, the primitive condition obtains in turtles (Gabe, 1970), and in mammals ancestrally (Griffiths, 1978). In contrast, the kidney in lepidosaurs, crocodiles, and birds is separated from the adrenal gland, and it has a more distant relationship to the gonads and gonoducts than to the metanephros (Gabe, 1970). The separation of the kidney from the adrenal gland is considered apomorphic (Table 1: A20).

(The loss of adrenal-kidney apposition and the closer relations of the gland to the gonads and gonoducts are diagnostic of Sauria on our tree (Fig. 3); however, on the Recent tree (Fig. 2) the apomorphic condition arose separately in lepidosaurs and archosaurs.)

\section{Fenestra Rotundum}

This character, cited by Gardiner as diagnostic of crocodiles, birds, and mammals, has been the source of considerable controversy, particularly in archosaur systematics. In our view, the problem is partly semantic, the source of much confusion being whether one is discussing the "hole or the doughnut". That is to say, the lateral aperture (fenestra rotundum sensu lato) is a hole in the braincase representing a remnant of the embryonic fissura metotica (viz. the gap between the developing otic capsule, basal plate, and occipital arch in the embryo). As such, it is a conserved aspect of the basic design of the tetrapod skull (Zeller, 1985) and, as a hole, it represents a plesiomorphic resemblance.

Evolutionary novelties appearing in this region are clarified if we instead focus on the doughnut, or more specifically, the means by which the anterior end of the embryonic fissura metotica may become subdivided during ontogeny. In so doing, some of the controversy surrounding the level of generality of this character may be put to rest.

The anterior end of the metotic fissure does not become subdivided ontogenetically in either turtles or Sphenodon, and this represents the ancestral amniote condition (Rieppel, 
1985). Squamates display an apomorphic condition, in contrast to Sphenodon and other tetrapods. The anterior end of the metotic fissure is divided from the only other part of the fissure remaining open in adults (through which the internal jugular vein and the glossopharyngeal and vagus nerves pass) by apposition of a hypertrophied portion of the otic capsule, which houses the ampulla of the posterior semicircular canal, to the lateral margin of the basal plate (Bellairs and Kamal, 1981). Our observations of hatchling squamates reveal that the medial wall of the otic capsule also possesses a vertical strut of bone which contacts the basal plate and which completes the division of the metotic fissure internally. Snakes differ in that the lateral and posterior ampullae of the semicircular canals are smaller than in other lepidosaurs (as a glance through the foramen magnum will attest). Thus, the external, ampulla-to-basal plate contact fails to take place, so that the "vertical strut" is the only portion of the otic capsule involved in division of the metotic fissure (Kamal, 1971). Since the outgroups display neither condition, only part of this transformation can be polarized unambiguously; the vertical strut descending from the otic capsule to contact the basal plate, and thus enclosing the anterior part of the metotic fissure, can be considered a synapomorphy of squamates. However, without making assumptions about the relationship of snakes to other squamates, the generality of an external contact between the ampulla and basal plate cannot be determined. It is noteworthy, however, that the metotic fissure is anomalously broad in hatchling snakes, compared to the condition seen in other lepidosaurs; in fact, the uppermost corner of the fissure may often remain open in adults (Bellairs and Kamal, 1981). This, combined with evidence indicating that snakes are scleroglossan squamates (Estes et al., 1988), leads us to conclude that the condition in snakes is secondary (contra Rieppel, 1985). Indeed, we believe that in failing to contact the basal plate laterally, this region of the otic capsule displays yet another derived character, an example of paedomorphosis, in the highly modified chondrocranium of snakes.

Crocodiles and birds also possess separate, apomorphic conditions by which the anteriormost portions of their metotic fissures become enclosed. In crocodiles, a cartilaginous process (the subscapular process) arises from the lateral surface of the occipital arch, just above the hypoglossal foramen, and extends forward beneath the auditory capsule, thus enclosing the anteriormost portion of the fissura metotica from below and behind (de Beer, 1937). In contrast, in birds a neomorph condensation, the metotic cartilage, arises independently at a position opposite the center of the metotic fissure and below the canalicular portion of the otic capsule. The metotic cartilage subsequently fuses to the base of the occipital arch, from which it projects forward and outward beneath the auditory capsule. Its dorsal margin subsequently fuses to the side wall of the canalicular portion of the otic capsule and its ventromedial margin to the lateral edge of the basal plate, thus enclosing the anteriormost portion of the metotic fissure from the side and behind (de Beer, 1937; Frank, 1954). De Beer (1937) considered the avian metotic cartilage and crocodilian subscapular process to be homologous. We agree with Rieppel (1985) that de Beer's conclusion was predicated on process assumptions rather than on anatomical particulars. De Beer accepted a decidedly Haeckelian view of the "vertebral origin" of the skull, and thus interpreted these structures as "ribs".

Mammals display yet another means by which the anteriormost portion of the metotic fissure becomes enclosed during ontogeny. In therian mammals, "the ventral wall of the perilymphatic foramen extends backwards in the form of a process which fuses to the floor of the auditory capsule farther back" to enclose this portion of the fissura metotica 
(de Beer, 1937: 431). Monotremes present a problem in determining the level of generality of this state, because the process of the otic capsule is present in Tachyglossus (Kuhn, 1971), but absent in Ornithorhynchus (de Beer, 1937). No one doubts that the condition in Theria constitutes a synapomorphy (de Beer, 1937; Zeller, 1985). Thus, there are two equally parsimonious interpretations of the evolution of the processus recessus in mammals. One hypothesizes that the condition in Tachyglossus appeared in the common ancestor of mammals, subsequently becoming lost in Ornithorhynchus and further elaborated in therians (e.g., Tarsitano and Hecht, 1980). The other hypothesis emphasizes that the development of the process differs in Tachyglossus, thus indicating that it acquired its apomorphic similarities convergently from those seen in therian mammals (e.g., Kuhn, 1971; Zeller, 1985). Since we are interested in the phylogenetic implications of this character, we are concerned with explaining shared apomorphic similarities rather than dissimilarities. For example, the processus recessus arises from the otic capsule adjacent to the perilymphatic foramen in all mammals in which it is present, even though the timing of appearance and shape of the process may differ in Tachyglossus and therians (Kuhn, 1971). Any differences that may exist could well represent further modifications that took place in ancestral therians. Accordingly, we tentatively accept the thesis that the common ancestor of mammals possessed a processus recessus that enclosed the anteriormost remnant of the fissura metotica. We emphasize, however, that there is as yet no unambiguous answer to the question of the evolution of the processus recessus in mammals.

The previous discussion suggests there have been four different means by which the anteriormost remnant of the fissura metotica has become enclosed in amniotes: a process of the otic capsule in mammals; a process of the occipital arch in crocodiles; a separate metotic cartilage in birds; and simple apposition of the otic capsule and basal plate within lepidosaurs. Convergence is required in this character only if the apomorphic condition is thought of as a space or hole in the auditory region of the braincase. Convergent appearance of a hole is implicitly accepted in discussions of the character in such terms as recessus scalae tympani, apertura lateralis and medialis of the recessus scalae tympani, fenestra rotunda, fenestra pseudorotunda, aquaductus cochleae, and so forth, which have been applied to various portions of the anterior remnants of the embryonic metotic fissure. If, however, one conceives of the character as the presence or absence of various cartilaginous processes, neomorph condensations, or patterns of growth, all of which result in enclosure of the anterior space, then convergence need not be invoked in any instance. The same cannot be said for the presence of a secondary tympanic membrane.

The secondary tympanic membrane is stretched across the lateral remnant of the metotic fissure. The ancestral condition of absence is retained in turtles and Sphenodon, but the derived state obtains in squamates, crocodiles, birds, and mammals (aside from Ornithorhynchus). The membrane attaches ventrally to one of the new structures discussed above, but it is always at least partly attached dorsally to the otic capsule lateral to the perilymphatic foramen. Thus, we must admit it is possible that a secondary tympanic membrane was present in the common ancestor of mammals, birds, and crocodiles (although its ancestral ventral attachment would be indeterminable). It also may be possible that the various conditions of enclosure of the metotic fissure were the result of subsequent modifications of ontogeny within the three groups. In order to give Gardiner's hypothesis a fair trial, we tentatively accept this hypothesis in our reanalysis (Table 1: A21). 
(According to the Recent tree (Fig. 2), the secondary tympanic membrane diagnoses Thecodontia. Our hypothesis (Fig. 3) indicates that it arose independently in mammals, and in some pseudosuchians and ornithosuchians (e.g., Martin, 1983). Thus, it appears that an enclosed portion of the metotic fissure, together with its membranous covering, has appeared independently in mammals, squamates, crocodiles and birds. Also, it may be that the various conditions of metotic fissure enclosure happened independently within the three groups.)

\section{Thoracic Haemapophyses}

Gardiner (p. 216) diagnosed birds, crocodiles, and mammals as having "four or five haemapophyses on the thoracic vertebrae." We are not sure what he meant by this character, but if he was referring to ventromedial processes on the anterior thoracic and posterior cervical vertebrae, commonly referred to as hypapophyses, then this character can be considered an apomorphy only in crocodiles and birds (Table 1: A22).

(Hypapophyses on cervical and thoracic vertebrae diagnoses Archosauria on both trees (Figs 2, 3). However, ingroup hypotheses (e.g., Gauthier, 1984, 1986) make it clear that hypapophyses are not ancestral for Archosauria. On the contrary, these structures appear to have evolved independently within crocodylomorphs (i.e., eusuchians) and within maniraptoran coelurosaurian theropods. They evolved yet again in snakes and varanoid lizards.)

\section{Hooked 5th Metatarsal.}

Gardiner listed the presence of a hooked fifth metatarsal as an amniote synapomorphy. This conclusion was based on Goodrich's (1916) observation that a hooked fifth metatarsal is present in turtles, crocodiles, and lepidosaurs (the fifth digit is lost early in the ontogeny of the bird foot). Gardiner (p. 219) denied Goodrich's conclusion as to the level of generality of this character, because he claimed to have observed a hooked fifth metatarsal in "such mammals as the marsupial anteater, the wombat, Dasyurus, Ailuropa, and Myrmecophaga." We have examined the fifth metatarsal in all these taxa, except the marsupial anteater. They were uniformly like monotremes and all other mammals in that none of them displayed a medially inflected proximal end of the fifth metatarsal. Thus, we will accept Goodrich's (1916) conclusion that this apomorphy is shared by turtles, crocodiles, and lepidosaurs alone among tetrapods. We record the condition in birds, which lack a fifth digit, as not applicable, $\mathrm{N}$ (Table 1: A23).

(The Recent tree (Fig. 2) indicates that a hooked fifth metatarsal evolved at the level of Amniota and was lost in mammals. If not for fossils, one would think that this character diagnoses Reptilia (e.g., Goodrich, 1916). Ever since Broom (1921), however, it has been accepted that the fifth metatarsal was not hooked in reptiles ancestrally. On the contrary, a hooked fifth metatarsal appeared independently in turtles, lepidosaurs, and archosauromorphs (Gauthier, 1984).)

\section{Pro-atlas}

De Beer (1937) discussed the fate of the body of the pro-atlas vertebra in his consideration of occipital segmentation in tetrapods, an attribute that has figured in some analyses of amniote phylogeny (e.g., Benton, 1985). The pro-atlas pleurocentrum fuses with the pleurocentrum of the atlas in mammals and lepidosaurs, but it fuses with 
the posterior end of the skull in turtles, crocodiles, and birds (de Beer, 1937). Neither condition is present in lissamphibian ontogeny (Table 1: A24).

(The history of pro-atlas pleurocentrum fusion is equivocal on both trees (Figs 2, 3). If fusion to the posterior end of the skull is diagnostic of Amniota, then the conditions in lepidosaurs and mammals evolved convergently. Alternatively, if the fusion is to the atlas, then the derived state evolved independently in turtles and archosaurs.)

\section{HuXIEY's Foramen}

Huxley's foramen, not to be confused with the stapedial foramen, is present at the distal end of the extracolumella, and it is formed medially by the processes dorsalis and intercalary, and dorsolaterally by the laterohyal. It is found in lepidosaurs (although absent in most lineages of squamates), crocodiles (Benton, 1985), and birds (de Beer, 1937). The ancestral condition, absence of that foramen, is typical of lissamphibians, turtles, and mammals (Table 1: A25). More primitive mammals, such as Tachyglossus (Huxley, 1869) and Didelphis (de Beer, 1937), show no evidence of a foramen in the distal part of the hyoid arch.

(Huxley's foramen evolved at the level of Sauria on our tree (Fig. 3). On the Recent tree (Fig. 2), the foramen originated separately in lepidosaurs and archosaurs.)

\section{6. Отоцітнs}

Maisey (1987) recently analyzed vertebrate otoliths. He concluded that "monocrystalline otoliths characterize dipnoans and tetrapods; these are aragonite primitively, but are also calcite in amniotes (exclusively so in birds and mammals)." Accordingly, we will consider exclusively calcite otoliths a shared apomorphy of birds and mammals (Table 1: $\Lambda 26)$.

(Calcite replaced aragonite independently in mammals and birds according to our classification (Fig. 3). On the Recent tree, the history of this character is equivocal. It is either diagnostic of Thecodontia and reversed in crocodiles, or it is convergent in birds and mammals.)

\section{Proximal Quadrate $\Lambda$ rticulation}

According to the work summarized by Martin (1983), crocodiles and birds must be considered apomorphic compared to other extant tetrapods in that they possess a bipartite quadrate articulation (Table 1: $\Lambda 27$ ); one of the proximal facets is associated with the prootic and laterosphenoid anteriorly and the other with the prootic posteriorly (i.e., the quadrate cotylus lies at the anterior base of the parocciput).

(The bipartite quadrate articulation diagnoses $\Lambda$ rchosauria on the Recent tree (Fig. 2). Fossil archosaurs indicate otherwise; this character appears to have arisen twice, because it is absent in all extinct pseudosuchians, including the early crocodylomorph Terrestrisuchus (Crush, 1984), and in all extinct ornithosuchians, including the early bird Archaeopteryx (Walker, 1985).)

\section{Siphonial System}

Martin (1983) also pointed out that birds and crocodiles are alone among tetrapods in possessing a siphonial system, which enters the pneumatic articular bone via the foramen aerosum (Table 1: $\Lambda 28$ ). 
(Archosauria is diagnosed on both trees (Figs 2,3) by the presence of a siphonial system.)

\section{Postmandibular Branchial Arches}

According to Edgeworth (1935) and de Beer (1937), turtles, lepidosaurs, crocodiles, and birds are alone among tetrapods in possessing fewer than three postmandibular branchial arches. Three or more pairs of branchial arches are retained in mammals ancestrally and lissamphibians (Table 1: A29).

(The reduction in the number of postmandibular branchial arches delimits Reptilia on our tree (Fig. 3). However, the Recent hypothesis (Fig. 2) suggests that the reduction took place at the level of $A$ mniota and that mammals re-evolved the branchial arches.)

\section{Mandibular Fenestra}

$\Lambda$ fenestra between the dentary and postdentary bones is present in crocodiles and in birds ancestrally, and absent in turtles, lepidosaurs, and lissamphibians. Mammalian postdentary bones are too modified to be interpreted in this respect; they may have achieved their present state through either condition. Based on this distribution, absence is considered ancestral and presence derived (Table 1: A30).

(According to the Recent hypothesis (Fig. 2), a mandibular fenestra could diagnose either thecodonts or archosaurs. We hypothesize that the character diagnoses archosauriform archosauromorphs (Fig. 3).)

\section{Large Premaxillary Bone.}

The premaxilla is small in lissamphibians, lepidosaurs, and turtles, and large in crocodiles, birds, and most mammals. In mammals, the enlargement is primarily in the postnasal process. That process is small in crocodiles and absent altogether in birds. In crocodiles and birds, the enlargement of the premaxilla is a result of prenarial expansion. In the absence of other historical data, it remains possible that one of these states is a transformation of the other and we are obliged to treat enlargement of the premaxilla as a potential synapomorphy.

(On the Recent tree (Fig, 2), a large premaxilla diagnoses 'Thecodontia, whereas we suggest (Fig. 3) that it arose independently in mammaliamorph cynodonts and at the level of Archosauromorpha. The fossil data indicate that expansion of the postnarial process is entirely separate from the prenarial expansion in archosauromorphs, and the two transformations are treated as separate characters in Table 2.)

\section{Internasal Progess of Premaxillary Bone}

$\Lambda$ nasal process of the premaxilla is present in lissamphibians, lepidosaurs, and birds, but absent in turtles, crocodiles, and mammals. The premaxilla itself appears to be absent in extant dipnoans. The former state is considered ancestral and the latter derived (Table 1: A32).

(On the Recent tree, reduction of the premaxillary nasal process diagnoses Euamniota and reverses in birds (Fig. 2). Based on evidence from fossils, however, extant turtles, crocodiles, and mammals have acquired the derived condition separately.) 


\section{Nasal Bones}

The nasal bones are shorter than the frontals in lissamphibians, primitive lepidosaurs, and turtles, and they are longer than the frontals in crocodiles, birds, and mammals ancestrally. The former condition is considered ancestral and the latter derived (Table 1: A33).

(Elongate nasal bones diagnose Thecodontia on the Recent tree (Fig. 2), but we hypothesize (Fig. 3) that the derived condition arose separately within synapsids and archosauromorphs.)

\section{External NARES}

The maxilla forms the posterior margin of the external nares in lissamphibians, lepidosaurs, turtles, and birds, but it does not contribute to the narial margin in crocodiles and mammals. The former condition is considered ancestral and the latter derived (Table 1: A34).

(The history of this character is ambiguous on the Recent tree (Fig. 2), which suggests that it arose either at the level of Thecodontia and reversed in birds, or originated independently in crocodiles and mammals. We hypothesize (Fig. 3) that the character arose independently in mammaliamorph cynodonts and in ancestral Archosauromorpha, and reversed within birds.)

\section{Postorbital Bone}

The postorbital bone is absent in lissamphibians, mammals, and birds, and present in crocodiles, turtles, and lepidosaurs. The former condition is considered ancestral and the latter derived (Table 1: $\Lambda 35$ ).

(The appearance of a postorbital bone is diagnostic of $A$ mniota on the Recent tree (Fig. 2), but its history among thecodonts is equivocal. It either reversed at the level of Thecodontia and re-evolved in crocodiles, or it was lost independently in mammals and birds. According to our hypothesis (Fig. 3), however, a postorbital is present in amniotes ancestrally, and it has been lost independently in mammaliamorph cynodonts and birds.)

\section{Postparietal Bone}

An azygous postparietal bone is absent in lissamphibians, lepidosaurs, and turtles, but present, at least in the embryo, in crocodiles, birds, and mammals (the latter group may occasionally possess additional centers of ossification in the "postparietal region" (de Beer, 1937)). The former condition is considered ancestral and the latter derived (Table 1: A36).

(The presence of a discrete postparietal bone at some stage of ontogeny is considered a synapomorphy of Thecodontia on the Recent tree (Fig. 2). In the context of the more complete knowledge of the relevant taxa afforded by fossils, however, it is clear that paired postparietals are ancestral for tetrapods. Further, they fuse to form an azygous element in synapsids and archosauromorphs, and have been lost independently in lissamphibians, turtles, and lepidosaurs.)

\section{Separate Exoccipital in Adults}

Adult lissamphibians, lepidosaurs, crocodiles, and birds do not have separate 
exoccipitals, but the exoccipitals remain separate from the opisthotics in turtles and mammals. The former condition is considered ancestral and the latter derived (Table 1: $\Lambda 37)$.

(The history of exoccipital-opisthotic fusion is ambiguous on the Recent tree (Fig. 2). Failure to fuse either arose in Euamniota ancestrally and subsequently reversed in mammals, or it arose once in turtles and once in mammals. In the context of fossil evidence, however, the polarity is reversed, with separation being ancestral and fusion apomorphic. Thus, according to our amniote hypothesis of relationships (Fig. 3), fusion between the exoccipital and opisthotic took place within lepidosauromorphs and in archosaurs. Fusion also characterizes Lissamphibia.)

\section{VOMERS}

The vomers are paired in lissamphibians and crocodiles, and in lepidosaurs ancestrally, but they are at least partly fused together in adult turtles, birds, and mammals. The former condition is considered ancestral and the latter derived (Table 1 : A38).

(At least partial vomer fusion in adults is diagnostic of Euamniota and reversed in crocodiles on the Recent tree (Fig. 2). Our hypothesis (Fig. 3), however, indicates that this character arose within turtles, and in cynodonts and theropod ornithosuchians.)

\section{Secondary Palate Conststing of Maxilla and Palatine}

A secondary palate is absent ancestrally in lissamphibians, lepidosaurs, turtles, and birds, but it is present in crocodiles and mammals. The former condition is considered ancestral and the latter derived (Table 1: A39).

(The diagnostic content of a secondary palate consisting of maxillae and palatines is ambiguous according to the Recent hypothesis (Fig. 2). It either arose in thecodonts and reversed in birds, or it arose separately in crocodiles and mammals. Our hypothesis (Fig. 3 ) indicates that the apomorphic condition appeared separately within crocodylomorphs and twice within Synapsida, in Therocephalia and in Cynodontia.)

\section{ECTOPTERYGOID}

$\Lambda \mathrm{n}$ ectopterygoid is absent in lissamphibians, except for a few caecilians which have a bone in the palate that has been given that name (M. H. Wake, pers. comm.). The ectopterygoid is also absent in turtles, and birds, but present in crocodiles, lepidosaurs, and in adult monotremes and embryonic therians (Presley and Steel, 1978). The former condition is considered plesiomorphic and the latter derived (Table 1: A40).

(The appearance of an ectopterygoid is equivocal on the Recent tree (Fig. 2). It either evolved in amniotes ancestrally and reversed in turtles and birds, or it appeared independently in lepidosaurs, mammals, and crocodiles. Fossils indicate that lissamphibians have lost the ectopterygoid, consequently reversing the polarity of this character. Thus, its absence diagnoses the group turtles + captorhinids, and birds among ornithosuchians, while its presence during at least part of ontogeny in the rest of the amniotes constitutes a plesiomorphy.)

\section{Marginal Teeth}

Marginal teeth are present in lissamphibians, lepidosaurs, and crocodiles, but they are 
absent in turtles and birds. Both states are present in mammals, and that taxon is scored as retaining the primitive state ancestrally (see "Data and Methods of Analysis"). The presence of marginal teeth in amniotes is considered ancestral, their absence derived (Table 1: A41).

(Loss of marginal teeth occurred separately in turtles and birds on the Recent tree (Fig. 2) and on our hypothesis (Fig. 3). Teeth are retained in some extinct birds.)

\section{INTERCLAVICLE}

An interclavicle is absent in lissamphibians and birds, but it is present in lepidosaurs, turtles, crocodiles, and phylogenetically primitive mammals. The former condition is considered ancestral and the latter derived (Table 1: $\Lambda 42$ ).

(The evolution of an interclavicle is diagnostic of Amniota and reversed in birds on the Recent tree (Fig. 2). In the context of our evidence (Fig. 3), however, the polarity is reversed, and interclavicle loss diagnoses lissamphibians, all ornithosuchian archosaurs except Euparkeria and Ornithosuchidae, and theriiform mammals.)

\section{Head of Frgur}

The head of the femur is not inturned relative to the shaft of the bone in lissamphibians, lepidosaurs, turtles, and crocodiles, whereas it is inturned, thus lying nearly in the plane of the distal femoral condyles, in mammals and birds. The former condition is considered ancestral and the latter derived (Table 1: A43).

(The history of this character is equivocal on the Recent tree (Fig. 2); it either arose in thecodonts ancestrally and reversed in crocodiles, or it evolved independently in birds and mammals. According to our hypothesis (Fig. 3), an inturned femoral head arose within cynodonts and independently within both ornithosuchian and pseudosuchian archosaurs.)

\section{Acetabulum}

The acetabulum is a solid plate of bone in lissamphibians (except caecilians), lepidosaurs, and turtles, but the acetabulum is incompletely ossified, and perforate, in birds and crocodiles. The former condition is considered ancestral and the latter derived (Table 1: A44). Mammals display both conditions, with the acetabulum being imperforate in therians and perforate in Tachyglossus. Mammals have been scored as state 0 in Table 1 (see "Data and Method of Analysis" for reasoning).

(Perforation of the acetabulum is equivocal on the Recent tree (Fig. 2). It could have evolved in thecodonts and reversed in therians, or it could have evolved independently in Tachyglossus and archosaurs. Our hypothesis (Fig. 3) indicates that this character evolved independently in Tachyglossus, crocodiles, and within ornithosuchian archosaurs.)

\section{Calganeal Tuber}

A calcaneal tuber is absent in lissamphibians, turtles, lepidosaurs, and birds, and present in crocodiles and mammals. The former condition is considered ancestral and the latter derived (Table 1: A45).

(According to the Recent hypothesis (Fig. 2), the history of the calcaneal tuber is ambiguous. It could have arisen in thecodonts ancestrally, and reversed in birds, or it 
could have evolved independently in crocodiles and mammals. Our hypothesis (Fig. 3) indicates that it arose within cynodonts and separately in archosauromorphs.)

\section{Phalangeal Formula of Third Toe}

Lissamphibians, turtles, and mammals ancestrally have three phalanges in pedal digit III, but at least four phalanges are present in lepidosaurs, crocodiles, and birds. The former condition is considered ancestral and the latter derived (Table 1: $\Lambda 46$ ).

(Lepidosaurs and archosaurs would be diagnosed by the convergent addition of phalanges to this toe, according to the Recent hypothesis (Fig. 2). Our knowledge of fossil tetrapods suggests that the polarity of this character should be reversed. Thus, lissamphibians, turtles, and mammals have each lost phalanges in this digit independently of one another, and the four phalanges present in other amniotes represent plesiomorphy rather than apomorphy.)

\section{Phalangeal Formula of Fourth Toe}

There are fewer than five phalanges in pedal digit IV in all extant tetrapods except lepidosaurs and birds, which have five. The former condition is considered ancestral and the latter derived (Table 1: A47).

(The possession of five phalanges in the fourth toe evolved independently in lepidosaurs and birds according to the Recent tree (Fig. 2). Consideration of fossil tetrapods leads to the conclusion that the polarity of this character should be reversed, with five phalanges being ancestral and a reduced number apomorphic. Lissamphibians, turtles, mammals, and crocodiles have all acquired the derived state independently.)

\section{Phalangeal. Formula of Fifth Toe}

There are either two or three phalanges in pedal digit $\mathrm{V}$ in lissamphibians, turtles, lepidosaurs, and mammals ancestrally; however, a metatarsal is all that remains of this digit in birds (embryos) and crocodiles. The former condition is considered ancestral and the latter derived (Table 1: A48).

(This character diagnoses archosaurs on the Recent tree (Fig. 2). Ingroup hypotheses indicate several reductions/losses of the fifth pedal digit in archosauriforms, and it is clear that the condition seen in crocodiles and birds was acquired independently.)

\section{Length of Fourth Toe Relative to Third}

The fourth pedal digit is longer than the third in lissamphibians and lepidosaurs, whereas the third toe is longer than the fourth in turtles, mammals ancestrally, crocodiles, and birds. The former is considered the ancestral condition and the latter derived (Table 1: $\Lambda 49$ ).

(The apomorphic condition diagnoses Euamniota according to the Recent hypothesis (Fig. 2). Our tree (Fig. 3) suggests, however, that a reduced fourth toe appeared independently within archosauromorphs and therapsids, and in turtles.)

\section{Spine Tables}

Spine tables, or distal expansions of neural spine apices in the posterior cervicals and 
anterior trunk vertebrae, are absent in lissamphibians, lepidosaurs, turtles, and mammals, but present in large crocodiles and in birds. The former condition is considered ancestral and the latter derived (Table 1: A50).

(Spine tables diagnose archosaurs on both trees (Figs 2, 3).)

\section{Supra-acetabular Buttress}

The dorsal edge of the acetabulum is not buttressed in lissamphibians, turtles, lepidosaurs, and crocodiles, but there is a thickened dorsal rim, the supra-acetabular buttress, in birds and phylogenetically primitive mammals. The former condition is considered ancestral and the latter derived (Table 1: A51).

(This character is equivocal on the Recent tree (Fig. 2), because it could be considered diagnostic of 'Thecodontia and reversed in crocodiles, or it could have evolved separately in birds and mammals. Our hypothesis suggests that the supra-acetabular buttress evolved separately, once in Therapsida and once within ornithosuchians (Gauthier, 1984, 1986).)

\section{Cervical Ribs}

Cervical ribs are absent in lissamphibians (caecilians may be exceptional; $M$. $H$. Wake, pers. comm.) and turtles, but they are presrnt in lepidosaurs, crocodiles, birds. They are also present in mammals embryonically, but in all mammals the postaxial ribs differentiate and then quickly fuse to their corresponding vertebrac, and in therians the axial rib fuses as well. $A$ bsence of cervical ribs is considered ancestral and their presence derived (Table 1: $A 52$ ).

(The diagnostic level of cervical ribs is ambiguous on the Recent tree (Fig. 2). Evidence from extinct tetrapods suggests that the polarity of this transformation should be reversed, with cervical ribs being present ancestrally, and their absence apomorphic. Our hypothesis (Fig. 3) indicates that cervical ribs have been lost within Testudines.)

\section{Parietals}

Parietal bones are paired in lissamphibians, lepidosaurs, and turtles, but they are fused together in adult crocodilians and birds. Both states occur in mammals, so in keeping with the strategy argued in the section "Data and Methods of Analysis", the primitive condition is attributed to that taxon. The paired condition is considered ancestral and the fused state derived (Table $1: \Lambda 53$ ).

(Parietal fusion diagnoses Archosauria on the Recent tree (Fig. 2), but according to our hypothesis (Fig. 3) this character is considered to have arisen separately within Crocodylomorpha and within birds.)

\section{Fossil Data}

We list below characters derived from the work of Gauthier (1984) on reptiles and Rowe (1986a) on synapsids. Limited space dictates the brevity of these character descriptions, and we advise the reader to refer to those publications for further documentation. 


\section{Skull:}

1. Premaxilla. Small (0) or large, forming most of tip of snout (1).

2. Premaxilla. Without $(0)$ or with (1) prominent postnarial process contacting nasal to exclude maxilla from external naris.

3. Septomaxilla. Small and confined to naris $(0)$ or large and with facial process excluding maxilla from narial margin (1).

4. Nasal. Shorter $(0)$, or longer (1), than frontal.

5. Prefronial. Present $(0)$ or absent (1).

6. Prefrontal posifrontal. In contact $(0)$ or not, so that frontal participates in orbital margin (1).

7. Prefrontal-postorbital. Separated $(0)$ or in contact (1).

8. Fronial margins. Subparallel $(0)$ or with lappets entering orbital margin (1).

9. Postfrontal. Present (0) or absent (1).

10. Posifronlal. Large (0) or small (1).

11. Postorbital. Lies lateral to parietal $(0)$ or overlies parietal so that temporal musculature originates on its dorsal surface (1).

12. Postorbilal. Present $(0)$ or absent (1).

13. Postorbital - squamosal. In contact $(0)$ or separated (1).

14. Postorbital. Temporal process extends about halfway back $(0)$ or to posterior end of skull (1).

15. Postorbital region of skull relative to preorbital length. Subequal (0), preorbital longer (1) or postorbital longer (2).

16. Parietal foramen. Present (0) or absent (1).

17. Parielal foramen. Large (0) or small (1).

18. Parietal boss. Absent (0) or present (1).

19. Sagillal crest. Absent (0) or present (1).

20. Temporal muscles on parietal table. Originate ventrolaterally $(0)$ or dorsolaterally (1).

21. Lamboidal crest. Absent (0) or present (1).

22. Postparielal. Paired $(0)$ or fused (1).

23. Supratemporal. Present (0) or absent (1).

24. Supratemporal. Large and in contact with postorbital $(0)$ or small and separated from postorbital (1).

25. Tabular. Present (0) or absent (1).

26. Tabular opistholic. Contact present $(0)$ or absent (1).

27. Maxilla - prefrontal. Separated by lacrimal $(0)$ or in contact (1).

28. Maxilla. Excluded from (0), or broadly enters into (1), subtemporal fenestra.

29. Maxilla palatal shelves. Small and broadly separated (0), or prominent and appressed on midline to form secondary palate (1).

30. Maxilla ventral margin. Horizontal $(0)$ or bowed ventrally (1).

31. Antorbital fenesira. $\Lambda$ bsent $(0)$ or present (1).

32. Antorbital fossa. Absent (0) or present (1).

33. Lacrimal. Separates maxilla and nasal and enters margin of naris $(0)$ or maxilla contacts nasal and excludes lacrimal from naris (1).

34. Jugal. Extends posteriorly to middle of lower temporal fenestra $(0)$ or extends nearly to posterior end of skull (1).

35. Upper temporal fenestra. Absent (0), large (1) or small (2).

36. Upper temporal fenestra. Absent (0), dorsolaterally (1), or dorsally (2), oriented.

37. Lower temporal fenestra. Absent $(0)$ or present $(1)$. 
38. Lower temporal fenestra. $\Lambda$ bsent (0), or opens laterally (1) or dorsally (2).

39. Ventral margin of postorbilal region of skull. Convex (0) or horizontal (1).

40. Ventral margin of postorbital region of skull. Convex $(0)$ or concave (1).

41. Post-temporal fenestra. Small (0), large (1) or medium (2)-sized in adults.

42. Basipterygoid articulation. Present (0) or absent (1).

43. Vomer. Paired (0) or fused partly or entirely (1).

44. Palatine palalal processes. Separated posteriorly (0) or appressed on midline throughout length forming secondary palate (1).

45. Palatine. Flat dorsally (0) or with ascending process contacting frontal (1).

46. Palatine. Undifferentiated $(0)$ or with separate palatal and choanal rami (1).

47. Pyriform recess. Extends anteriorly to posterior (0), or nearly to anterior (1), ends of palatines.

48. Squamesal. Extends to (0), or broadly separated from (1), ventral margin of skull.

49. Squamosal external acoustic meatus. $\Lambda$ bsent (0), shallow (1), or deeply folded (2).

50. Squamosal- quadratojugal posterior margins. Vertical (0) or bowed (1) (viz. otic notch present).

51. Quadratojugal. Borders lower cheek region $(0)$ or confined ro rear corner of temporal fenestra (1).

52. Quadratojugal processes. Long anterior and short dorsal rami (0) or short anterior and long dorsal rami (1).

53. Quadratojugal. Overlaps (0), or fits into notch in posteroventral edge of (1), squamosal.

54. Pterygoid -parasphenoid behind transverse process. Broadly separated $(0)$ or appressed and clasping (1).

55. Ectoplerygoid. Present (0) or absent (1) as discrete element in adult.

56. Tabular. Large $(0)$ or small (1).

57. Suborbital fenestra. Absent $(0)$, small (1), or large (2).

58. Ectopterygoid - palaiine. In contact or nearly so medially $(0)$ or broadly separated (1).

59. Quadrate-quadratojugal. Exposed posteriorly (0) or largely covered by squamosal (1).

60. Quadrale-squamosal peg-in-socket articulation. Absent $(0)$ or present (1).

61. Quadrate. Abuts against flat $(0)$, or notched (1), ventral surface of squamosal.

62. Quadrate. Covered by squamosal (0), or exposed (1), laterally.

63. Quadrate plerygoid contact. Present $(0)$ or absent (1).

64. Quadrate stapedial process. Absent (0) or present (1) (viz. crus longus present).

65. Epiplerygoid dorsal process. Narrow (0) or broad (1).

66. Epipterygoid. Does not reach main body of parietal (0) or broadly overlaps parietal dorsally $(1)$.

67. Epipterygoid-frontal conlact. Absent $(0)$ or present (1).

68. Stapes. Stout $(0)$ or rod-like (1).

69. Stapes dorsal process. With (0), or without (1) osseous connection to paroccipital process of opisthotic.

70. Craniomandibular joint. Even with occiput $(0)$, or posterior (1), or anterior (2), to occiput.

71. Laterosphenoid ossification. Absent $(0)$ or present (1).

72. Cochlear promontorium. Absent $(0)$ or present (1).

73. Prootic flange --quadrate ramus of pterygoid contact. Absent (0) or present (1). 
74. Supraoccipital. Broad and without anterior crista $(0)$ or narrow and with anterior crista (1).

75. Supraoccipital. Lateral margins vertical $(0)$ or expanded laterally to roof posttemporal fenestra dorsally (1).

76. Opisthotic. Paroccipital process undivided distally (0) or bifurcate (1).

77. Opisthotic. Paroccipital process tapering (0) or expanded (1) distally.

78. Opisthotic. Paroccipital process extending laterally $(0)$ or posteriorly (1).

79. Opisthotic. Paroccipital process extending horizontally (0) or ventrolaterally (1).

80. Exoccipilal. Separate (0), or fused with opisthotic (1), in adult.

81. Occipital condyle. Formed from basioccipital and exoccipitals $\{0\rangle$ or primarily by exoccipitals (1).

82. Occipilal condyle. Exoccipitals in contact on condyle mid-dorsally $(0)$ or not in contact (1).

83. Occipilal condyle. Basioccipital forms part (0), or most (1), or none (2), of condyle.

84. Anterior lamina of petrosal. Absent (0) or present (1).

85. Occipital plate outline. Straight $(0)$ or emarginated parasagittally (1) (viz. Wshaped).

\section{Mandible:}

86. Mandible. Transects lateral (0), or middle (1), portion of adductor fossa.

87. Mandibular fenestra. Absent (0) or present (1).

88. Masseteric fossa. Absent (0) or present (2).

89. Dentary angular process. Absent $(0)$ or present (1).

90. Dentary. Broadly separated from $(0)$ or closely approaching or contacting (1) craniomandibular joint.

91. Dentary - squamosal. Not in contact (0) or articulating (1).

92. Dentary-coronoid-surangular proflle. Subhorizontal (0) or strongly arched dorsally (1)

93. Anteromost portions of anterior postdentary bones. Partly (0), or entirely (1), lie within Meckelian sulcus.

94. Meckelian sulcus. Broadly open (0) or narrow (1) posteriorly.

95. Dentary coronoid process. $\Lambda$ bsent $(0)$ or present (1).

96. Dentary coronoid process. Rear margin vertical (0) or emarginate (1).

97. Dentary coronoid process. Projects to (0) or above (1) level of zygoma.

98. Angular. Ventral margin rounded $(0)$ or keeled (1).

99. Angular. Short $(0)$ or tall (1) dorsoventrally.

100. Coronoid. Anterior coronoid present $(0)$ or absent (1).

101. Angular. Not incised (0), or moderately (1) or deeply (2) incised.

102. Angular reflected lamina. Absent (0) or present (1).

103. Angular. Not ring-like (0) or ring-like (1).

104. Retroarticular process. Medially (0), or posteriorly (1), directed.

105. Retroarticular process. Horizontal (0) or downcurved (1).

\section{Dentition:}

106. Maxillary and dentary teeth. In shallow (0), or deep (1), sockets.

107. Tooth altachment. By attachment bone (0) or periodontal ligament (1).

108. Serrated teeth. Absent (0) or present $(1)$.

109. Upper--lower tooth rows. End at same level posteriorly $(0)$ or dentary row terminates anterior to maxillary tooth row (1).

110. Premaxillary teeth. Implanted in shallow $(0)$, or deep (1), sockets. 
Table 2

Synapomorphies of the major groups of amniotes.

\begin{tabular}{|c|c|c|c|c|c|c|c|c|c|c|}
\hline & Ancestor & Casea & $\begin{array}{l}\text { Ophiat- } \\
\text { odon }\end{array}$ & $\begin{array}{l}\text { Edapho- } \\
\text { saurus }\end{array}$ & $\begin{array}{l}\text { Sphenac- } \\
\text { odontinae }\end{array}$ & $\begin{array}{c}\text { Biarmo- } \\
\text { suchia }\end{array}$ & $\begin{array}{l}\text { Dino- } \\
\text { cephalia }\end{array}$ & $\begin{array}{l}\text { Gorgon- } \\
\text { opsia }\end{array}$ & $\begin{array}{l}\text { Dicyn- } \\
\text { odontia }\end{array}$ & $\begin{array}{l}\text { Thero- } \\
\text { cephalia }\end{array}$ \\
\hline 1 & 0 & 0 & 0 & 0 & 0 & 0 & 0 & 0 & 0 & 0 \\
\hline 2 & 0 & 0 & 0 & 0 & 0 & 0 & 0 & 0 & 0 & 0 \\
\hline 3 & 0 & 0 & 0 & 0 & 0 & 1 & 1 & 1 & 1 & 1 \\
\hline 4 & 0 & 1 & 1 & 0 & 1 & I & 1 & 1 & 1 & 1 \\
\hline 5 & 0 & 0 & 0 & 0 & 0 & 0 & 0 & 0 & 0 & 0 \\
\hline 6 & 0 & 1 & 1 & 1 & 1 & 1 & 1 & 1 & 1 & 1 \\
\hline 7 & 0 & 0 & 0 & 0 & 0 & 0 & 0 & 0 & 0 & 0 \\
\hline 8 & 0 & 0 & 0 & 1 & 1 & 1 & 1 & 1 & 0 & 0 \\
\hline 9 & 0 & 0 & 0 & 0 & 0 & 0 & 0 & 0 & 0 & 0 \\
\hline 10 & 0 & 0 & 0 & 0 & 0 & 0 & 0 & 0 & 1 & 1 \\
\hline 11 & 0 & 0 & 0 & 0 & 0 & 0 & 1 & 1 & 1 & 1 \\
\hline 12 & 0 & 0 & 0 & 0 & 0 & 0 & 0 & 0 & 0 & 0 \\
\hline 13 & 0 & $?$ & 0 & ? & 0 & 0 & 0 & 0 & 0 & 1 \\
\hline 14 & 0 & ? & 1 & $?$ & 1 & 1 & 1 & 1 & 1 & 0 \\
\hline 15 & 0 & 0 & 1 & 0 & 1 & l & 1 & 1 & 2 & 2 \\
\hline 16 & 0 & 0 & 0 & 0 & 0 & 0 & 0 & 0 & $0 / 1$ & 0 \\
\hline 17 & 0 & 0 & 0 & 0 & 0 & 0 & 0 & 0 & $0 / 1 / \mathrm{N}$ & 1 \\
\hline 18 & 0 & 0 & 0 & 0 & 0 & 1 & 1 & 1 & 1 & 0 \\
\hline 19 & 0 & 0 & 0 & 0 & 0 & 0 & 0 & 0 & $0 / 1$ & 1 \\
\hline 20 & 0 & 0 & 0 & 0 & 0 & 0 & 0 & 0 & $0 / 1$ & 1 \\
\hline 21 & 0 & 0 & 0 & 0 & 0 & 0 & 1 & 1 & 1 & 1 \\
\hline 22 & 0 & 1 & 1 & 1 & 1 & 1 & 1 & 1 & 1 & 1 \\
\hline 23 & 0 & 0 & 0 & 0 & 0 & 0 & $0 / 1$ & 1 & 1 & 1 \\
\hline 24 & 0 & 0 & 0 & 0 & 0 & 0 & $1 / \mathrm{N}$ & $\mathrm{N}$ & $\mathbf{N}$ & $\mathrm{N}$ \\
\hline 25 & 0 & 0 & 0 & 0 & 0 & 0 & 0 & 0 & 0 & 0 \\
\hline 26 & 0 & 0 & 0 & 0 & 0 & 0 & 0 & 0 & 0 & 0 \\
\hline 27 & 0 & 0 & 0 & 0 & 0 & l & 1 & 1 & 1 & l \\
\hline 28 & 0 & 0 & 0 & 0 & 0 & 0 & 0 & 0 & 0 & $0 / 1$ \\
\hline 29 & 0 & 0 & 0 & 0 & 0 & 0 & 0 & 0 & $0 / \mathrm{N}$ & $0 / 1$ \\
\hline 30 & 0 & 0 & 1 & 1 & 1 & 1 & 1 & 1 & $1 / \mathbf{N}$ & 1 \\
\hline 31 & 0 & 0 & 0 & 0 & 0 & 0 & 0 & 0 & 0 & 0 \\
\hline 32 & 0 & 0 & 0 & 0 & 0 & 0 & 0 & 0 & 0 & 0 \\
\hline 33 & 0 & 0 & 0 & 0 & 1 & 1 & 1 & 1 & $1 / 0$ & 1 \\
\hline 34 & 0 & 0 & 0 & 0 & 0 & 0 & 0 & 0 & 0 & 0 \\
\hline 35 & 0 & 0 & 0 & 0 & 0 & 0 & 0 & 0 & 0 & 0 \\
\hline 36 & 0 & 0 & 0 & 0 & 0 & 0 & 0 & 0 & 0 & 0 \\
\hline 37 & 0 & 1 & 1 & l & 1 & I & 1 & l & 1 & 1 \\
\hline 38 & 0 & 1 & 1 & 1 & 1 & 1 & 2 & 2 & 2 & 2 \\
\hline 39 & 0 & 0 & $\mathbf{N}$ & $\mathrm{N}$ & $N$ & $\mathrm{~N}$ & $\mathrm{~N}$ & $\mathbf{N}$ & $N$ & $\mathrm{~N}$ \\
\hline 40 & 0 & 0 & 1 & 1 & 1 & 1 & 1 & 1 & 1 & 1 \\
\hline 41 & 0 & 0 & 0 & 0 & 0 & 0 & 0 & 0 & 0 & 0 \\
\hline 42 & 0 & 0 & 0 & 0 & 0 & 1 & 1 & 1 & 1 & 1 \\
\hline 43 & 0 & 0 & 0 & 0 & 0 & 0 & 0 & 1 & $0 / 1$ & $0 / 1$ \\
\hline 44 & 0 & 0 & 0 & 0 & 0 & 0 & 0 & 0 & 0 & $0 / 1$ \\
\hline 45 & 0 & 0 & 0 & 0 & 0 & 0 & 0 & 0 & 0 & 0 \\
\hline 46 & 0 & 0 & 0 & 0 & 0 & 0 & 0 & 0 & 1 & l \\
\hline 47 & 0 & 0 & 0 & 0 & 0 & 0 & 0 & 0 & 0 & 0 \\
\hline 48 & 0 & 0 & 0 & 0 & 0 & 0 & 0 & 0 & 0 & 0 \\
\hline 49 & 0 & 0 & 0 & 0 & 0 & l & 1 & 1 & 1 & 1 \\
\hline 50 & 0 & 0 & 0 & 0 & 0 & 0 & 0 & 0 & 0 & 0 \\
\hline 51 & 0 & 0 & 0 & 1 & 1 & 1 & 1 & 1 & 1 & 1 \\
\hline 52 & 0 & 0 & 0 & $\mathrm{~N}$ & $\mathrm{~N}$ & $\mathbf{N}$ & $\mathrm{N}$ & $\mathbf{N}$ & $\mathbf{N}$ & $\mathrm{N}$ \\
\hline 53 & 0 & 0 & 0 & 0 & 0 & 0 & 0 & 0 & 0 & $1 / 0$ \\
\hline 54 & 0 & 0 & 0 & 0 & 0 & 0 & 0 & 0 & $0 / \mathrm{N}$ & 0 \\
\hline 55 & 0 & 0 & 0 & 0 & 0 & 0 & 0 & 0 & $0 / 1$ & 0 \\
\hline 56 & 0 & 0 & 0 & 0 & 0 & 0 & 0 & 0 & 0 & 0 \\
\hline 57 & 0 & 0 & 0 & 0 & 0 & 0 & 0 & 0 & $0 / 1$ & 2 \\
\hline
\end{tabular}


Table 2 -contd.

\begin{tabular}{|c|c|c|c|c|c|c|c|c|c|c|}
\hline & Ancestor & Casea & $\begin{array}{l}\text { Ophint- } \\
\text { odon }\end{array}$ & $\begin{array}{l}\text { Edapho- } \\
\text { saurts }\end{array}$ & $\begin{array}{l}\text { Sphenac- } \\
\text { odontinac }\end{array}$ & $\begin{array}{l}\text { Biarmo- } \\
\text { suchia }\end{array}$ & $\begin{array}{l}\text { Dino- } \\
\text { cephalia }\end{array}$ & $\begin{array}{c}\text { Gorgon- } \\
\text { opsia }\end{array}$ & $\begin{array}{l}\text { Dicyn- } \\
\text { odontia }\end{array}$ & $\begin{array}{l}\text { Thero- } \\
\text { cephaliaa }\end{array}$ \\
\hline 58 & $\overline{0}$ & 0 & 0 & 0 & 0 & 0 & 0 & 0 & $0 / \mathrm{N}$ & 1 \\
\hline 59 & 0 & 0 & 0 & 0 & 0 & 0 & 0 & 1 & 1 & 1 \\
\hline 60 & 0 & 0 & 0 & 0 & 0 & 0 & 1 & 1 & 1 & 1 \\
\hline 61 & 0 & 0 & 0 & 0 & 0 & 0 & 0 & 0 & 0 & 0 \\
\hline 62 & 0 & 0 & 0 & 0 & 0 & 0 & 0 & 0 & 0 & 0 \\
\hline 63 & 0 & 0 & 0 & 0 & 0 & 0 & 0 & 0 & 0 & 0 \\
\hline 64 & 0 & 0 & 0 & 0 & 0 & 0 & 0 & 0 & 0 & 0 \\
\hline 65 & 0 & 0 & 0 & 0 & 0 & 0 & 0 & 0 & 0 & $0 / 1$ \\
\hline 66 & 0 & 0 & 0 & 0 & 0 & 0 & 0 & 1 & I & 1 \\
\hline 67 & 0 & 0 & 0 & 0 & 0 & 0 & 0 & 0 & 0 & 0 \\
\hline 68 & 0 & 0 & 0 & 0 & 0 & 0 & 0 & 0 & 0 & 0 \\
\hline 69 & 0 & 0 & 0 & 0 & 0 & 1 & 1 & 1 & 1 & I \\
\hline 70 & 0 & 1 & 1 & 1 & 1 & 0 & 0 & 0 & 0 & 0 \\
\hline 71 & 0 & 0 & 0 & 0 & 0 & 0 & 0 & 0 & 0 & 0 \\
\hline 72 & 0 & 0 & 0 & 0 & 0 & 0 & 0 & 0 & 0 & 0 \\
\hline 73 & 0 & 0 & 0 & 0 & 0 & 0 & 0 & 0 & 0 & 1 \\
\hline 74 & 0 & 0 & 0 & 0 & 0 & 0 & 0 & 0 & 0 & 0 \\
\hline 75 & 0 & 1 & ? & 1 & 1 & 1 & 1 & 1 & 1 & 1 \\
\hline 76 & 0 & 0 & 0 & 0 & 0 & 0 & 0 & 0 & 0 & 0 \\
\hline 77 & 0 & 0 & 0 & 0 & 0 & 0 & 0 & 0 & 0 & 0 \\
\hline 78 & 0 & 0 & 0 & 0 & 0 & 0 & 0 & 0 & 0 & 0 \\
\hline 79 & 0 & 0 & 0 & 0 & 1 & 1 & 1 & 1 & 1 & 1 \\
\hline 80 & 0 & 0 & 0 & 0 & 0 & 0 & 0 & 0 & 0 & 0 \\
\hline 81 & $0 / 1$ & 0 & 0 & 0 & 0 & 0 & 0 & 0 & 0 & 0 \\
\hline 82 & 0 & 0 & 0 & 0 & 0 & 0 & 0 & 0 & 0 & 0 \\
\hline 83 & 0 & 0 & 0 & 0 & 0 & 0 & 0 & 0 & 0 & 0 \\
\hline 84 & 0 & 0 & 0 & 0 & 0 & 0 & 0 & 0 & 0 & 0 \\
\hline 85 & 0 & 0 & 0 & 0 & 0 & 0 & 0 & 0 & 0 & 0 \\
\hline 86 & 0 & 0 & 0 & 0 & 0 & 0 & 0 & 0 & 0 & 0 \\
\hline 87 & 0 & 0 & 0 & 0 & 0 & 0 & 0 & 0 & 1 & 1 \\
\hline 88 & 0 & 0 & 0 & 0 & 0 & 0 & 0 & 0 & 0 & 0 \\
\hline 89 & 0 & 0 & 0 & 0 & 0 & 0 & 0 & 0 & 0 & 0 \\
\hline 90 & 0 & 0 & 0 & 0 & 0 & 0 & 0 & 0 & 0 & 0 \\
\hline 91 & 0 & 0 & 0 & 0 & 0 & 0 & 0 & 0 & 0 & 0 \\
\hline 92 & 0 & 0 & 0 & 1 & 1 & 1 & I & $\mathrm{N}$ & $1 / \mathbf{N}$ & $N$ \\
\hline 93 & 0 & 0 & 0 & 0 & 0 & 0 & 0 & 0 & 0 & 0 \\
\hline 94 & 0 & 0 & 0 & 0 & 0 & 0 & 0 & 0 & 0 & 0 \\
\hline 95 & 0 & 0 & 0 & 0 & 0 & 0 & 0 & 1 & $1 / 0$ & 1 \\
\hline 96 & $\mathrm{~N}$ & $N$ & $\mathrm{~N}$ & $\mathrm{~N}$ & $\mathrm{~N}$ & $\mathrm{~N}$ & $\mathrm{~N}$ & 0 & $\mathrm{~N}$ & 0 \\
\hline 97 & $\mathrm{~N}$ & $N$ & $\mathrm{~N}$ & $N$ & $\mathrm{~N}$ & $\mathrm{~N}$ & $\mathrm{~N}$ & 0 & $0 / N^{\prime}$ & 0 \\
\hline 98 & 0 & 1 & 1 & 1 & 1 & 1 & 1 & 1 & l & 1 \\
\hline 99 & 0 & 0 & 1 & 1 & 1 & 1 & 1 & 1 & 1 & 1 \\
\hline 100 & 0 & 0 & 0 & 0 & 0 & 1 & 1 & 1 & 1 & 1 \\
\hline 101 & 0 & 0 & 0 & 0 & 0 & $i$ & 1 & $i$ & 1 & 1 \\
\hline 102 & 0 & 0 & 0 & 0 & 1 & 1 & 1 & 1 & 1 & 1 \\
\hline 103 & 0 & 0 & 0 & 0 & 0 & 0 & 0 & 0 & 0 & 0 \\
\hline 104 & 0 & 1 & 0 & 0 & 1 & 1 & 1 & 1 & 1 & 1 \\
\hline 105 & 0 & 0 & 0 & 0 & 1 & 1 & 1 & 1 & 1 & 1 \\
\hline 106 & 0 & 0 & 0 & 0 & 0 & 0 & 0 & 0 & 0 & 0 \\
\hline 107 & 0 & 0 & 0 & 0 & 0 & 0 & 0 & 0 & 0 & 0 \\
\hline 108 & 0 & 0 & 0 & 0 & 1 & 1 & 1 & 1 & 1 & 1 \\
\hline 109 & 0 & 0 & 0 & 0 & 0 & 0 & 0 & 0 & 0 & 0 \\
\hline 110 & 0 & 0 & 0 & 0 & 1 & 1 & 1 & 1 & $1 / N$ & 1 \\
\hline 111 & 0 & $0 / 1$ & 0 & 1 & 0 & 0 & 0 & 0 & $0 / 1$ & 0 \\
\hline 112 & 0 & 0 & 0 & 0 & 1 & 1 & 1 & 1 & $1 / N$ & 1 \\
\hline 113 & 0 & 0 & 0 & $N$ & $i$ & 1 & 1 & 1 & $\mathrm{l} / \mathrm{N}$ & 1 \\
\hline 114 & 0 & 0 & 0 & $\mathrm{~N}$ & 0 & 1 & 1 & 1 & $\mathrm{l} / \mathrm{N}$ & 1 \\
\hline 115 & 0 & 0 & 0 & $\mathrm{~N}$ & 0 & 0 & 0 & 0 & 0 & $0 / 1$ \\
\hline 116 & 0 & 0 & 0 & $\mathrm{~N}$ & 1 & 1 & 1 & 1 & $1 / \mathrm{N}$ & 1 \\
\hline
\end{tabular}


Table 2-contd

\begin{tabular}{|c|c|c|c|c|c|c|c|c|c|c|}
\hline & Ancestor & Casea & $\begin{array}{c}\text { Ophiac- } \\
\text { odon }\end{array}$ & $\begin{array}{l}\text { Edapho- } \\
\text { saurus }\end{array}$ & $\begin{array}{l}\text { Sphenac- } \\
\text { odontinae }\end{array}$ & $\begin{array}{l}\text { Biarmo- } \\
\text { suchia }\end{array}$ & $\begin{array}{c}\text { Dino- } \\
\text { cephalia }\end{array}$ & $\begin{array}{l}\text { Gorgon- } \\
\text { opsia }\end{array}$ & $\begin{array}{l}\text { Dicyn- } \\
\text { odontia }\end{array}$ & $\begin{array}{l}\text { Thero- } \\
\text { cephalia }\end{array}$ \\
\hline 117 & 0 & 0 & 0 & 0 & 0 & 0 & 0 & 0 & $0 / \mathrm{N}$ & $0 / \mathbf{N}$ \\
\hline 118 & 0 & 0 & 0 & 0 & 0 & 0 & 0 & 0 & 0 & 0 \\
\hline 119 & 0 & 0 & 0 & 0 & 1 & I & l & 1 & $1 / N$ & 1 \\
\hline 120 & 0 & 0 & $?$ & 0 & 1 & 1 & 1 & 1 & 1 & 1 \\
\hline 121 & 0 & 0 & $?$ & 0 & 0 & 0 & 0 & 0 & 1 & 1 \\
\hline 122 & 0 & 0 & $?$ & 0 & 0 & I & 1 & 1 & $1 / \mathrm{N}$ & 1 \\
\hline 123 & 0 & 0 & 0 & 0 & 0 & 0 & 0 & 0 & 1 & 0 \\
\hline 124 & 0 & 0 & 0 & 0 & 0 & 1 & 1 & 1 & I & 1 \\
\hline 125 & 0 & 0 & 0 & 0 & 0 & 0 & 0 & 0 & 0 & 0 \\
\hline 126 & 0 & 0 & 0 & 0 & 0 & 0 & 0 & 0 & 0 & 0 \\
\hline 127 & 0 & 0 & 0 & 0 & 0 & 1 & 1 & 1 & 1 & 1 \\
\hline 128 & 0 & 0 & 0 & 0 & 0 & 0 & 1 & 1 & 1 & 1 \\
\hline 129 & 0 & 0 & 0 & 0 & 0 & 0 & 0 & 0 & 0 & 0 \\
\hline 130 & 0 & 1 & $?$ & $?$ & 1 & 1 & 1 & 1 & 1 & 1 \\
\hline 131 & 0 & 0 & 1 & $?$ & 0 & 0 & 0 & 0 & 1 & 1 \\
\hline 132 & 0 & 0 & 0 & 0 & 0 & 0 & 0 & 0 & 0 & 0 \\
\hline 133 & 0 & 0 & 0 & 0 & 0 & 0 & 0 & 0 & 1 & 1 \\
\hline 134 & 0 & 0 & 0 & 0 & 0 & 0 & 0 & 0 & 0 & 0 \\
\hline 135 & 0 & 0 & 0 & $?$ & 0 & 0 & 0 & 0 & 1 & 1 \\
\hline 136 & 0 & 0 & 0 & $?$ & 0 & 0 & 0 & 1 & 1 & 1 \\
\hline 137 & 0 & 0 & 0 & $?$ & 0 & 0 & 0 & 1 & 1 & 1 \\
\hline 138 & 0 & 0 & 0 & 0 & 0 & 0 & 0 & 0 & 0 & 0 \\
\hline 139 & 0 & 0 & 0 & 0 & 1 & 1 & l & 1 & 1 & 1 \\
\hline 140 & 0 & 0 & 0 & 0 & 0 & 0 & 0 & 0 & 0 & 0 \\
\hline 141 & 0 & 1 & 0 & 1 & 1 & 1 & 1 & 1 & 1 & 1 \\
\hline 142 & 0 & 0 & 0 & 0 & 0 & 1 & 1 & 1 & 1 & 1 \\
\hline 143 & 0 & 0 & 0 & 0 & 0 & 0 & 0 & 0 & 0 & 0 \\
\hline 144 & 0 & 0 & 0 & 0 & 0 & 0 & 0 & 0 & 0 & 0 \\
\hline 145 & 0 & 0 & 0 & 0 & 0 & 0 & 0 & 0 & 0 & 0 \\
\hline 146 & 0 & 0 & 0 & 0 & 0 & 1 & l & 1 & 1 & 1 \\
\hline 147 & 0 & 0 & 0 & 0 & 0 & 1 & 1 & 1 & 1 & 1 \\
\hline 148 & 0 & 1 & 1 & 1 & 1 & 1 & 1 & 1 & 1 & 1 \\
\hline 149 & 0 & 0 & 0 & 0 & 0 & 0 & 0 & 0 & 0 & 0 \\
\hline 150 & 0 & 0 & 0 & 0 & 0 & 0 & 0 & 0 & 0 & 0 \\
\hline 151 & 0 & 0 & 0 & 0 & 0 & 0 & 0 & 0 & 0 & 1 \\
\hline 152 & 0 & 0 & 0 & 0 & 0 & 1 & 1 & 1 & 1 & $\mathbf{N}$ \\
\hline 153 & 0 & 0 & 0 & 0 & 0 & 0 & 0 & 0 & 1 & 1 \\
\hline 154 & 0 & 0 & 0 & 0 & 0 & 0 & 0 & 0 & 0 & 0 \\
\hline 155 & 0 & 0 & 0 & 0 & 0 & 0 & 0 & 0 & 0 & 0 \\
\hline 156 & 0 & 0 & 0 & 0 & 0 & 0 & 0 & 0 & 0 & 0 \\
\hline 157 & 0 & 0 & ? & 0 & 0 & 1 & 1 & 1 & 1 & 1 \\
\hline 158 & 0 & 0 & ? & 0 & 0 & 0 & 0 & 0 & 0 & 0 \\
\hline 159 & 0 & 0 & 0 & 0 & 0 & 0 & 0 & 0 & 0 & 0 \\
\hline 160 & 0 & 0 & 0 & 0 & 0 & 0 & 0 & 0 & 0 & 0 \\
\hline 161 & 0 & 0 & 0 & 0 & 0 & 0 & 0 & 0 & I & 1 \\
\hline 162 & 0 & 0 & 0 & 0 & 0 & 0 & 0 & 0 & 0 & 0 \\
\hline 163 & 0 & 1 & 0 & 1 & 0 & 0 & I & 1 & 1 & 1 \\
\hline 164 & 0 & 0 & 0 & 0 & 0 & 0 & 0 & 0 & 0 & 0 \\
\hline 165 & 0 & 0 & 0 & 0 & 0 & 0 & 0 & 0 & 1 & 1 \\
\hline 166 & 0 & 0 & 0 & 0 & 0 & 1 & 1 & 1 & 1 & 1 \\
\hline 167 & 0 & 0 & 0 & 0 & 0 & 0 & 1 & 1 & 1 & 1 \\
\hline 168 & 0 & 0 & 0 & 0 & 0 & 0 & 0 & 0 & 0 & 0 \\
\hline 169 & 0 & 0 & 0 & 1 & 1 & 1 & 1 & 1 & 1 & 1 \\
\hline 170 & 0 & 0 & 0 & 0 & 0 & 0 & 0 & 0 & 0 & 0 \\
\hline 171 & 0 & 0 & I & 1 & 1 & 1 & 1 & 1 & 1 & 1 \\
\hline 172 & 0 & 0 & 0 & 0 & 0 & 0 & 0 & 0 & 1 & 1 \\
\hline 173 & 0 & 0 & 0 & 0 & 0 & 0 & 0 & 0 & 0 & 0 \\
\hline 174 & 0 & 0 & 0 & 0 & 0 & 0 & 0 & 0 & 0 & 0 \\
\hline
\end{tabular}


Table 2-contd.

\begin{tabular}{|c|c|c|c|c|c|c|c|c|c|c|}
\hline & Ancestor & Casea & $\begin{array}{l}\text { Ophia- } \\
\text { odon }\end{array}$ & $\begin{array}{l}\text { Edapho- } \\
\text { saurus }\end{array}$ & $\begin{array}{l}\text { Sphenac- } \\
\text { odontinac }\end{array}$ & $\begin{array}{l}\text { Biarmo- } \\
\text { suchia }\end{array}$ & $\begin{array}{c}\text { Dino- } \\
\text { cephalia }\end{array}$ & $\begin{array}{c}\text { Gorgon- } \\
\text { opsia }\end{array}$ & $\begin{array}{l}\text { Dicyn- } \\
\text { odontia }\end{array}$ & $\begin{array}{l}\text { Thero- } \\
\text { cephalia }\end{array}$ \\
\hline 175 & 0 & 0 & 0 & 0 & 0 & 0 & 0 & 0 & 0 & 0 \\
\hline 176 & 0 & 0 & 0 & 0 & 0 & 0 & 0 & 0 & 0 & 0 \\
\hline 177 & 0 & 0 & 0 & 0 & 0 & 1 & I & 1 & 1 & 1 \\
\hline 178 & 0 & 0 & 0 & 0 & 0 & 1 & 1 & 1 & 1 & 1 \\
\hline 179 & 0 & 0 & 0 & 0 & 0 & 1 & 1 & 1 & 1 & 1 \\
\hline 180 & 0 & 0 & 0 & 0 & 0 & 0 & 0 & 0 & 0 & 0 \\
\hline 181 & 0 & 0 & 0 & 0 & 0 & 0 & 0 & 0 & 0 & 0 \\
\hline 182 & 0 & 0 & 0 & 0 & 0 & 0 & 0 & 0 & 1 & $0 / 1$ \\
\hline 183 & 0 & 0 & 0 & 0 & 0 & 0 & 0 & 0 & 0 & 0 \\
\hline 184 & 0 & 0 & 0 & 0 & 0 & 2 & 2 & 2 & 2 & 2 \\
\hline 185 & 0 & 0 & 0 & 0 & 0 & 0 & 0 & 0 & 0 & 0 \\
\hline 186 & 0 & 0 & 0 & 0 & 0 & 1 & l & 1 & 1 & 1 \\
\hline 187 & 0 & 0 & 0 & 0 & 0 & 1 & 1 & l & 1 & 1 \\
\hline 188 & 0 & 0 & 0 & 0 & 0 & 1 & 1 & 1 & 2 & 2 \\
\hline 189 & 0 & 0 & 0 & 0 & 0 & 1 & I & 1 & 1 & 1 \\
\hline 190 & 0 & 0 & 0 & 0 & 0 & 1 & 1 & 1 & 1 & 1 \\
\hline 191 & 0 & 0 & 0 & 0 & 0 & 0 & 0 & 0 & 0 & 0 \\
\hline 192 & 0 & 0 & 0 & 0 & 0 & 0 & 0 & 0 & 0 & 0 \\
\hline 193 & 0 & 0 & 0 & 0 & 0 & 0 & 0 & 0 & 0 & 0 \\
\hline 194 & 0 & 0 & 0 & 0 & 0 & 1 & 1 & 1 & I & 1 \\
\hline 195 & 0 & 0 & 0 & 0 & 0 & 0 & 0 & 0 & 0 & 0 \\
\hline 196 & 0 & 0 & 0 & 0 & 0 & $\mathrm{~N}$ & $\mathrm{~N}$ & $\mathbf{N}$ & $\mathbf{N}$ & $\mathrm{N}$ \\
\hline 197 & 0 & 0 & 0 & 0 & 0 & 0 & 0 & 0 & 0 & 0 \\
\hline 198 & 0 & 0 & 0 & 0 & 0 & 0 & 0 & 0 & 0 & $0 / 2$ \\
\hline 199 & 0 & 0 & 0 & 0 & 0 & 0 & 0 & 0 & 0 & 0 \\
\hline 200 & 0 & 0 & 0 & 0 & 0 & 1 & 0 & 1 & 1 & 1 \\
\hline 201 & 0 & 0 & 0 & 0 & 0 & 0 & 0 & 0 & 0 & 0 \\
\hline 202 & 0 & 0 & 0 & 0 & 0 & 0 & 0 & 0 & 0 & 0 \\
\hline 203 & 0 & 0 & 0 & 0 & 0 & 0 & 0 & 0 & 0 & 0 \\
\hline 204 & 0 & 0 & 0 & 0 & 0 & 0 & 0 & 0 & 0 & 0 \\
\hline 205 & 0 & 0 & 0 & 0 & 0 & 0 & 0 & 0 & 0 & 0 \\
\hline 206 & 0 & 0 & 0 & 0 & 0 & 1 & $0 / 1$ & 1 & I & 1 \\
\hline 207 & 0 & 0 & 0 & 0 & 0 & 1 & 1 & 1 & 1 & 1 \\
\hline
\end{tabular}

\begin{tabular}{|c|c|c|c|c|c|c|c|c|c|c|}
\hline & $\begin{array}{l}\text { Procyno- } \\
\text { suchus. }\end{array}$ & $\begin{array}{c}\text { Thrinax- } \\
\text { odon }\end{array}$ & $\begin{array}{c}\text { Diadem- } \\
\text { adon }\end{array}$ & $\begin{array}{c}\text { Exaerel- } \\
\text { odon }\end{array}$ & $\begin{array}{c}\text { Trityl- } \\
\text { odontidae }\end{array}$ & $\begin{array}{l}\text { Morganuc- } \\
\text { odontidae }\end{array}$ & Mammalia & Testudines & $\begin{array}{l}\text { Capto- } \\
\text { rhinidae }\end{array}$ & $\begin{array}{l}\text { Araeo- } \\
\text { scelidia }\end{array}$ \\
\hline & $\cdots \quad \ldots \ldots$ & $\ldots$ & . $\quad \cdots-$ & $-\quad-\quad-$ & - - - - & $\cdots-\infty$ & $\cdots$ & $\cdots-\quad-\cdots$ & $-\ldots$ & $\cdots \quad-\quad-$ \\
\hline 1 & 0 & 0 & 0 & 0 & 0 & 0 & 0 & 0 & 0 & 0 \\
\hline 2 & 0 & 0 & 0 & 0 & 1 & 1 & 1 & 0 & 0 & 0 \\
\hline 3 & 1 & I & 1 & 1 & 1 & $?$ & $\mathrm{~N} / 0$ & 0 & 0 & 0 \\
\hline 4 & 1 & 1 & 1 & 1 & 1 & 1 & $1 / 0$ & 0 & 0 & 0 \\
\hline 5 & 0 & 0 & 0 & 0 & I & I & 1 & 0 & 0 & 0 \\
\hline 6 & $N$ & $\mathrm{~N}$ & $\mathrm{~N}$ & $\mathrm{~N}$ & $\mathrm{~N}$ & $\mathbf{N}$ & $\mathrm{N}$ & 1 & 1 & 1 \\
\hline 7 & 1 & 1 & 1 & 1 & $\mathrm{~N}$ & $\mathrm{~N}$ & $\mathrm{~N}$ & 0 & 0 & 0 \\
\hline 8 & 0 & 0 & 0 & 0 & 0 & 0 & 0 & 0 & 0 & 0 \\
\hline 9 & 1 & 1 & 1 & 1 & 1 & 1 & $1 / 0$ & 1 & 0 & 0 \\
\hline 10 & $N$ & $\mathrm{~N}$ & $\mathrm{~N}$ & $\mathrm{~N}$ & $N$ & $\mathrm{~N}$ & $\mathrm{~N} / 1$ & 0 & 0 & 0 \\
\hline 11 & 1 & l & 1 & 1 & $\mathrm{~N}$ & $\mathrm{~N}$ & $\mathrm{~N}$ & 0 & 0 & 0 \\
\hline 12 & 0 & 0 & 0 & 0 & 1 & 1 & $1 / 0$ & 0 & 0 & 0 \\
\hline 13 & 1 & 1 & 1 & 1 & $\mathrm{~N}$ & $\mathbf{N}$ & $\mathrm{N}$ & 0 & 0 & 0 \\
\hline 14 & 0 & 0 & 0 & 0 & $\mathrm{~N}$ & $N$ & $\mathrm{~N}$ & 0 & 0 & 0 \\
\hline 15 & 2 & 2 & 2 & 2 & 2 & 2 & $2 / 0 / 1$ & $0 / 2$ & 0 & l \\
\hline 16 & 0 & 0 & 0 & 1 & 1 & 1 & l & 1 & 0 & 0 \\
\hline 17 & 1 & 1 & 1 & $N$ & $\mathrm{~N}$ & $\mathrm{~N}$ & $\mathrm{~N}$ & $\mathrm{~N}$ & 0 & 0 \\
\hline 18 & 0 & 0 & 0 & 0 & 0 & 0 & 0 & 0 & 0 & 0 \\
\hline 19 & 1 & 1 & 1 & 1 & 1 & 1 & $1 / 0$ & $0 / 1$ & 0 & 0 \\
\hline 20 & 1 & l & 1 & 1 & 1 & 1 & I & $0 / 1$ & 0 & 0 \\
\hline
\end{tabular}


Table 2-contd.

\begin{tabular}{|c|c|c|c|c|c|c|c|c|c|c|}
\hline & $\begin{array}{c}\text { Procyno- } \\
\text { sturfius }\end{array}$ & $\begin{array}{c}\text { Thrinax- } \\
\text { odom }\end{array}$ & $\begin{array}{l}\text { Diadem- } \\
\text { odon }\end{array}$ & $\begin{array}{l}\text { Exaeret- } \\
\text { odon }\end{array}$ & $\begin{array}{c}\text { Trityl- } \\
\text { odontidae }\end{array}$ & $\begin{array}{l}\text { Morganuc- } \\
\text { odontidae }\end{array}$ & Mammalia & Testudines & $\begin{array}{l}\text { Capto- } \\
\text { rhinidae }\end{array}$ & $\begin{array}{l}\text { Araeo- } \\
\text { scelidia }\end{array}$ \\
\hline 21 & 1 & 1 & 1 & 1 & 1 & 1 & $1 / \mathrm{N}$ & 0 & 0 & 0 \\
\hline 22 & 1 & 1 & 1 & 1 & 1 & $?$ & $1 / 0 / \mathrm{N}$ & $\mathrm{N}$ & 0 & 0 \\
\hline 23 & 1 & 1 & 1 & 1 & 1 & l & 1 & $0 / 1$ & 0 & 0 \\
\hline 24 & $\mathbf{N}$ & $\mathrm{N}$ & $\mathrm{N}$ & $\mathbf{N}$ & $\mathbf{N}$ & $\mathrm{N}$ & $\mathrm{N}$ & $1 / N$ & 1 & 1 \\
\hline 25 & 0 & 0 & 0 & 0 & 0 & 1 & $1 / 0$ & 1 & l & 0 \\
\hline 26 & 0 & 0 & 0 & 0 & 0 & $\mathrm{~N}$ & $\mathrm{~N}$ & $N$ & $\mathrm{~N}$ & 1 \\
\hline 27 & 0 & 0 & 0 & 0 & $\mathrm{~N}$ & $\mathrm{~N}$ & $\mathrm{~N}$ & 0 & 0 & 0 \\
\hline 28 & 0 & 0 & 0 & 1 & 1 & 1 & l & 0 & 0 & 0 \\
\hline 29 & 0 & 1 & 1 & 1 & 1 & 1 & 1 & $0 / \mathrm{N}$ & 0 & 0 \\
\hline 30 & 1 & 1 & 1 & 0 & 1 & 0 & $0 / 1$ & $0 / 1$ & 0 & 0 \\
\hline 31 & 0 & 0 & 0 & 0 & 0 & 0 & 0 & 0 & 0 & 0 \\
\hline 32 & 0 & 0 & 0 & 0 & 0 & 0 & $0 / 1$ & 0 & 0 & 0 \\
\hline 33 & 1 & 1 & 1 & 1 & ! & I & 1 & $0 / \mathrm{N}$ & 0 & 0 \\
\hline 34 & 0 & I & 1 & 1 & 1 & $?$ & $1 / \mathrm{N}$ & 0 & 0 & 0 \\
\hline 35 & 0 & 0 & 0 & 0 & 0 & 0 & 0 & 0 & 0 & l \\
\hline 36 & 0 & 0 & 0 & 0 & 0 & 0 & 0 & 0 & 0 & 1 \\
\hline 37 & 1 & 1 & 1 & 1 & 1 & 1 & 1 & 0 & 0 & 1 \\
\hline 38 & 2 & 2 & 2 & 2 & 2 & 2 & 2 & 0 & 0 & 1 \\
\hline 39 & $\mathrm{~N}$ & $\mathbf{N}$ & $\mathbf{N}$ & $\mathrm{N}$ & $\mathbf{N}$ & $N$ & $\mathrm{~N}$ & 1 & 1 & 1 \\
\hline 40 & 1 & 1 & 1 & 1 & 1 & 1 & 1 & $\mathbf{N}$ & $\mathrm{N}$ & $\mathbf{N}$ \\
\hline 41 & 0 & 0 & 0 & 0 & 0 & 0 & $0 / \mathrm{N}$ & 1 & 1 & 1 \\
\hline 42 & 1 & 1 & 1 & 1 & 1 & 1 & 1 & 0 & 0 & 0 \\
\hline 43 & 1 & 1 & 1 & I & I & 1 & 1 & $0 / 1$ & 0 & 0 \\
\hline 44 & 0 & I & 1 & 1 & 1 & 1 & 1 & $0 / \mathrm{N}$ & 0 & 0 \\
\hline 45 & 0 & 0 & 0 & 0 & I & l & 1 & 0 & 0 & 0 \\
\hline 46 & 1 & 1 & 1 & 1 & 1 & 1 & 1 & 0 & 0 & 0 \\
\hline 47 & 0 & 0 & 0 & 0 & 0 & 0 & 0 & 0 & 0 & 0 \\
\hline 48 & 0 & 0 & 0 & 0 & 0 & 0 & 0 & 1 & 0 & 0 \\
\hline 49 & 1 & I & 2 & 2 & 2 & 2 & 2 & 0 & 0 & 0 \\
\hline 50 & 0 & 0 & 0 & 0 & 0 & 0 & $\mathrm{~N}$ & 1 & 0 & 0 \\
\hline 51 & 1 & 1 & 1 & 1 & 1 & 1 & $N$ & 0 & 0 & 0 \\
\hline 52 & $\mathrm{~N}$ & $\mathrm{~N}$ & $\mathrm{~N}$ & $\mathrm{~N}$ & $\mathrm{~N}$ & $\mathrm{~N}$ & $\mathrm{~N}$ & 0 & 0 & 0 \\
\hline 53 & 1 & 1 & 1 & 1 & 1 & 1 & $\mathrm{~N}$ & 0 & 0 & 0 \\
\hline 54 & 0 & 1 & l & 1 & 1 & 1 & 1 & 0 & 0 & 0 \\
\hline 55 & 0 & 0 & 0 & 1 & 1 & 1 & 1 & l & 1 & 0 \\
\hline 56 & 0 & 0 & 0 & 0 & 0 & $\mathrm{~N}$ & $N$ & $\mathbf{N}$ & $\mathrm{N}$ & 1 \\
\hline 57 & 0 & 0 & 0 & $\mathbf{N}$ & $\mathrm{N}$ & $N$ & 0 & 1 & 1 & 2 \\
\hline 58 & 0 & 0 & 0 & 0 & 0 & 0 & $\mathrm{~N}$ & $\mathrm{~N}$ & 0 & 0 \\
\hline 59 & 1 & 1 & 1 & 1 & 1 & l & $\mathrm{N}$ & 0 & 0 & 0 \\
\hline 60 & 1 & 1 & 1 & I & $\mathrm{N}$ & $?$ & $\mathrm{~N}$ & 0 & 0 & 0 \\
\hline 61 & 1 & i & 1 & 1 & 1 & 1 & $\mathrm{~N}$ & 0 & 0 & 0 \\
\hline 62 & 0 & 0 & 0 & 0 & 0 & 0 & $N$ & $0 / 1$ & 0 & 0 \\
\hline 63 & 0 & 0 & 1 & 1 & 1 & 1 & $N$ & 0 & 0 & 0 \\
\hline 64 & 0 & 0 & 0 & 0 & 1 & 1 & 1 & 0 & 0 & 0 \\
\hline 65 & I & 1 & l & 1 & 1 & 1 & $\mathrm{~N}$ & 0 & 0 & 0 \\
\hline 66 & l & 1 & 1 & 1 & 1 & 1 & $\mathrm{~N}$ & 0 & 0 & 0 \\
\hline 67 & 1 & 1 & 1 & 1 & 1 & I & $\mathrm{N}$ & 0 & 0 & 0 \\
\hline 68 & 0 & 0 & 0 & 0 & 0 & 0 & $N$ & 1 & 0 & 0 \\
\hline 69 & 1 & 1 & 1 & 1 & 1 & 1 & 1 & 0 & 0 & 0 \\
\hline 70 & 0 & 0 & 0 & 0 & 0 & 0 & $2 / 0$ & 0 & 0 & 0 \\
\hline 71 & 0 & 0 & 0 & 0 & 0 & 0 & 0 & 0 & 0 & 0 \\
\hline 72 & 0 & 0 & 0 & 0 & 0 & 1 & 1 & 0 & 0 & 0 \\
\hline 73 & 1 & I & 1 & 1 & 1 & 1 & $\mathrm{~N}$ & 0 & 0 & 0 \\
\hline 74 & 0 & 0 & 0 & 0 & 0 & 0 & 0 & 1 & 1 & 1 \\
\hline 75 & $\mathbf{N}$ & $\mathrm{N}$ & $\mathbf{N}$ & $\mathrm{N}$ & $\mathrm{N}$ & $\mathrm{N}$ & $\mathbf{N}$ & 0 & 0 & 0 \\
\hline 76 & 0 & 0 & 0 & 0 & 1 & 1 & $1 / \mathbf{N}$ & 0 & 0 & 0 \\
\hline 77 & 0 & 0 & 0 & 0 & 0 & 0 & $0 / \mathbf{N}$ & 0 & 0 & 0 \\
\hline 78 & 0 & 0 & 0 & 0 & 0 & 0 & 0 & 0 & 0 & 0 \\
\hline
\end{tabular}


Table 2 -contd.

\begin{tabular}{|c|c|c|c|c|c|c|c|c|c|c|}
\hline & $\begin{array}{l}\text { Procyno- } \\
\text { suchus }\end{array}$ & $\begin{array}{c}\text { Thrinax- } \\
\text { odon }\end{array}$ & $\begin{array}{c}\text { Diadem- } \\
\text { odon }\end{array}$ & $\begin{array}{l}\text { Exaeret- } \\
\text { odon }\end{array}$ & $\begin{array}{c}\text { Trityl- } \\
\text { odontidae }\end{array}$ & $\begin{array}{l}\text { Morganuc- } \\
\text { odontidae }\end{array}$ & Mammalia & Testudines & $\begin{array}{l}\text { Capto- } \\
\text { rhinidae }\end{array}$ & $\begin{array}{l}\text { Araeo- } \\
\text { scelidia }\end{array}$ \\
\hline 79 & 1 & 1 & 1 & 1 & 0 & 0 & $\mathrm{~N}$ & 0 & 0 & 0 \\
\hline 80 & 0 & 0 & 0 & 0 & 0 & 0 & 0 & 0 & 0 & 0 \\
\hline 81 & 1 & 1 & 1 & 1 & 1 & 1 & 1 & 0 & 0 & 0 \\
\hline 82 & 1 & 1 & 1 & 1 & 1 & 1 & 1 & 0 & 0 & 1 \\
\hline 83 & 2 & 2 & 2 & 2 & 2 & 2 & 2 & 0 & 0 & 1 \\
\hline 84 & 1 & l & 1 & 1 & 1 & l & 1 & 0 & 0 & 0 \\
\hline 85 & 0 & 1 & 1 & 1 & 1 & 1 & $1 / \mathrm{N}$ & 0 & 0 & 0 \\
\hline 86 & 1 & 1 & 1 & 1 & 1 & 1 & 1 & 0 & 0 & 0 \\
\hline 87 & 1 & $\mathrm{~N}$ & $\mathbf{N}$ & $\mathbf{N}$ & $\mathrm{N}$ & $\mathbf{N}$ & $\mathrm{N}$ & 0 & 0 & 0 \\
\hline 88 & 1 & 1 & 1 & 1 & 1 & 1 & 1 & 0 & 0 & 0 \\
\hline 89 & 0 & 1 & 1 & 1 & l & 1 & 1 & 0 & 0 & 0 \\
\hline 90 & 0 & 0 & 1 & 1 & l & 1 & 1 & 0 & 0 & 0 \\
\hline 91 & 0 & 0 & 0 & 0 & 0 & 1 & 1 & 0 & 0 & 0 \\
\hline 92 & $\mathrm{~N}$ & $\mathrm{~N}$ & $\mathbf{N}$ & $\mathrm{N}$ & $N$ & $\mathbf{N}$ & $\mathrm{N}$ & 0 & 0 & 0 \\
\hline 93 & 0 & 0 & 1 & 1 & 1 & 1 & $\mathrm{~N}$ & 0 & 0 & 0 \\
\hline 94 & 0 & 0 & 0 & 0 & l & 1 & $\mathbf{N}$ & 0 & 0 & 0 \\
\hline 95 & 1 & 1 & 1 & 1 & 1 & 1 & $1 / N$ & 0 & 0 & 0 \\
\hline 96 & 0 & 0 & 1 & 1 & 1 & 1 & $1 / N$ & 0 & 0 & 0 \\
\hline 97 & 0 & 1 & 1 & 1 & 1 & ] & $1 / N$ & 0 & 0 & 0 \\
\hline 98 & 1 & 1 & 1 & l & 1 & 1 & 1 & 0 & 0 & 0 \\
\hline 99 & 1 & 1 & 1 & 1 & 1 & 1 & 1 & 0 & 0 & 0 \\
\hline 100 & 1 & 1 & 1 & 1 & 1 & 1 & 1 & 1 & 1 & 1 \\
\hline 101 & 2 & 2 & 2 & 2 & 2 & 2 & 2 & 0 & 0 & 0 \\
\hline 102 & 1 & 1 & 1 & 1 & 1 & 1 & 1 & 0 & 0 & 0 \\
\hline 103 & 0 & 0 & 1 & 1 & 1 & 1 & 1 & 0 & 0 & 0 \\
\hline 104 & 1 & 1 & I & 1 & 1 & 1 & $\mathrm{~N}$ & l & 0 & 0 \\
\hline 105 & 1 & 1 & 1 & 1 & 1 & 1 & $\mathrm{~N}$ & 0 & 0 & 0 \\
\hline 106 & 0 & 0 & 1 & 1 & 1 & 1 & $1 / N$ & $\mathrm{~N}$ & 0 & 0 \\
\hline 107 & 0 & 0 & 1 & 1 & 1 & 1 & $1 / \mathrm{N}$ & $\mathrm{N}$ & 0 & 0 \\
\hline 108 & 0 & 0 & 0 & 0 & 0 & 0 & $0 / 1$ & $\mathrm{~N}$ & 0 & 0 \\
\hline 109 & 0 & 0 & 0 & 0 & 0 & 0 & $0 / \mathrm{N}$ & $\mathrm{N}$ & 0 & 0 \\
\hline 110 & 1 & 1 & 1 & 1 & 1 & 1 & $1 / N$ & $\mathrm{~N}$ & 0 & 0 \\
\hline 111 & 0 & 0 & 0 & 0 & 1 & 0 & $0 / 1$ & $\mathrm{~N}$ & 0 & 0 \\
\hline 112 & 1 & $\mathrm{~N}$ & $\mathrm{~N}$ & $\mathrm{~N}$ & $N$ & $\mathrm{~N}$ & $\mathrm{~N}$ & $\mathrm{~N}$ & 0 & 0 \\
\hline 113 & 1 & 1 & 1 & I & $N$ & 1 & $1 / N$ & $\mathbf{N}$ & 0 & 0 \\
\hline 114 & l & 1 & 1 & 1 & $\mathbf{N}$ & 1 & $1 / N$ & $\mathbf{N}$ & 0 & 0 \\
\hline 115 & 1 & 1 & 1 & 1 & 1 & 1 & $1 / 0 / \mathrm{N}$ & $N$ & 0 & 0 \\
\hline 116 & 1 & 1 & 1 & 1 & 1 & 1 & $1 / \mathrm{N} / 0$ & $\mathrm{~N}$ & 0 & 0 \\
\hline 117 & 0 & 0 & 1 & 1 & 0 & 0 & 0 & $\mathbf{N}$ & 0 & 0 \\
\hline 118 & 0 & 0 & 0 & 0 & 1 & 1 & $1 / 0$ & $\mathrm{~N}$ & 0 & 0 \\
\hline 119 & 1 & 1 & 1 & 1 & $N$ & 1 & $0 / 1$ & $\mathbf{N}$ & 0 & 0 \\
\hline 120 & 1 & 1 & 1 & 1 & 1 & 1 & 1 & $0 / 1$ & 0 & 0 \\
\hline 121 & 1 & 1 & 1 & 1 & 1 & 1 & 1 & $0 / 1$ & 0 & 0 \\
\hline 122 & 1 & 1 & 1 & $\mathrm{~N}$ & $\mathrm{~N}$ & $\mathrm{~N}$ & $\mathrm{~N}$ & $\mathrm{~N}$ & $\mathrm{~N}$ & 1 \\
\hline 123 & 1 & l & 1 & 1 & 1 & 1 & $\mathrm{~N}$ & 0 & 0 & 0 \\
\hline 124 & 1 & 1 & 1 & 1 & 1 & 1 & 1 & $0 / 1$ & 0 & 0 \\
\hline 125 & 0 & 1 & 1 & 0 & 0 & 1 & $1 / 0$ & 0 & 0 & 0 \\
\hline 126 & 0 & 0 & 0 & 0 & 0 & 0 & $0 / 1$ & 0 & 0 & 1 \\
\hline 127 & 1 & 1 & 1 & 1 & 1 & 1 & 1 & 1 & 0 & 0 \\
\hline 128 & 1 & 1 & 1 & 1 & 1 & 1 & 1 & 1 & 0 & 0 \\
\hline 129 & 0 & 0 & 0 & 1 & 1 & 1 & $1 / 0$ & 0 & 0 & 0 \\
\hline 130 & 1 & 1 & 1 & 1 & 1 & 1 & $1 / \mathrm{N}$ & 0 & 0 & 0 \\
\hline 131 & 1 & l & 1 & 1 & 1 & 1 & l & 1 & 1 & 1 \\
\hline 132 & 0 & 0 & 0 & 0 & 0 & 0 & 0 & 0 & 0 & 0 \\
\hline 133 & 1 & 1 & 1 & 1 & 1 & 1 & 1 & 0 & 0 & 0 \\
\hline 134 & 0 & 0 & 0 & 0 & 1 & 1 & $1 / 0$ & 0 & 0 & 0 \\
\hline 135 & 1 & 1 & 1 & l & 1 & l & 0 & 0 & 0 & 0 \\
\hline 136 & 1 & l & 1 & 1 & 1 & 1 & 1 & 0 & 0 & 0 \\
\hline 137 & 1 & 1 & 1 & 1 & 1 & 1 & 1 & 0 & 0 & 0 \\
\hline
\end{tabular}


Table 2 contd

\begin{tabular}{|c|c|c|c|c|c|c|c|c|c|c|}
\hline & $\begin{array}{l}\text { Procyno- } \\
\text { suchus }\end{array}$ & $\begin{array}{l}\text { Thrinax- } \\
\text { odon }\end{array}$ & $\begin{array}{l}\text { Diadem- } \\
\text { odon }\end{array}$ & $\begin{array}{c}\text { Exaerel- } \\
\text { odon }\end{array}$ & $\begin{array}{c}\text { Trityl- } \\
\text { odontidae }\end{array}$ & $\begin{array}{c}\text { Morganuc- } \\
\text { odontidae }\end{array}$ & Mammalia & Testudines & $\begin{array}{l}\text { Capto- } \\
\text { rhinidae }\end{array}$ & $\begin{array}{l}\text { Araeo- } \\
\text { scelidia }\end{array}$ \\
\hline 138 & 0 & 0 & 0 & 0 & 1 & 1 & 1 & 0 & 0 & 0 \\
\hline 139 & 1 & 1 & 1 & 1 & 1 & 1 & 1 & 1 & 0 & 1 \\
\hline 140 & 0 & 0 & 0 & 0 & 0 & 1 & 1 & 0 & 0 & 0 \\
\hline 141 & 1 & 1 & 1 & 1 & 1 & I & $1 / 0$ & 0 & 0 & 0 \\
\hline 142 & 1 & 1 & 1 & l & l & 1 & l & $\mathbf{N}$ & 0 & 0 \\
\hline 143 & 0 & 0 & 0 & 0 & 0 & 0 & 0 & $0 / \mathrm{N}$ & 0 & 0 \\
\hline 144 & 0 & 0 & 0 & 0 & 0 & 0 & 0 & $\mathrm{~N}$ & 0 & 0 \\
\hline 145 & 1 & 1 & 1 & i & 1 & 1 & 1 & $0 / \mathrm{N}$ & 0 & 0 \\
\hline 146 & 1 & 1 & 1 & 1 & 1 & 1 & $1 / N$ & 0 & 0 & 0 \\
\hline 147 & 1 & 1 & 1 & 1 & 1 & 1 & 1 & $\mathbf{N}$ & 0 & 0 \\
\hline 148 & 1 & 1 & 1 & 1 & 1 & 1 & 1 & 0 & 1 & 1 \\
\hline 149 & 0 & 0 & 0 & 0 & 0 & 0 & 0 & $\mathrm{~N}$ & 0 & 0 \\
\hline 150 & 0 & 0 & 0 & 1 & 1 & 1 & l & $\mathrm{N}$ & 0 & 0 \\
\hline 151 & 1 & 1 & 1 & 1 & 1 & 1 & 1 & 1 & 1 & 0 \\
\hline 152 & $\mathrm{~N}$ & $\mathbf{N}$ & $N$ & $\mathrm{~N}$ & $\mathrm{~N}$ & $N$ & $\mathrm{~N}$ & $\mathbf{N}$ & 0 & 0 \\
\hline 153 & 1 & 1 & 1 & 1 & 1 & 1 & $1 / N$ & $\mathbf{N}$ & 0 & 0 \\
\hline 154 & 0 & 0 & 0 & 0 & 0 & $?$ & $1 / \mathrm{N}$ & $0 / \mathbf{N}$ & 0 & 0 \\
\hline 155 & 0 & 0 & 0 & 0 & 0 & $?$ & $0 / \mathbf{N}$ & $\mathrm{N}$ & 0 & 0 \\
\hline 156 & 0 & 0 & 0 & 1 & 1 & $?$ & $1 / \mathrm{N}$ & $\mathbf{N}$ & 0 & 0 \\
\hline 157 & 1 & 1 & 1 & 1 & 1 & 1 & 1 & $\mathrm{~N}$ & $?$ & 0 \\
\hline 158 & 0 & 0 & 0 & 0 & 1 & 1 & 1 & $\mathrm{~N}$ & 0 & 0 \\
\hline 159 & 0 & 0 & 0 & 0 & 0 & 0 & $0 / 1$ & 0 & 0 & 1 \\
\hline 160 & 0 & 0 & 0 & 0 & 0 & 0 & $0 / 1$ & 1 & 0 & 0 \\
\hline 161 & 1 & 1 & I & 1 & 1 & 1 & $1 / N$ & 0 & 0 & 0 \\
\hline 162 & 0 & 0 & 0 & 0 & 0 & 0 & $0 / 1$ & 0 & 0 & 0 \\
\hline 163 & 1 & 1 & l & 1 & 0 & 0 & $0 / 1$ & 0 & 0 & 0 \\
\hline 164 & 0 & 0 & 0 & 0 & 0 & 0 & $0 / 1$ & 1 & 0 & 0 \\
\hline 165 & 1 & 1 & 1 & 1 & 1 & 1 & 1 & 0 & 0 & 0 \\
\hline 166 & 1 & 1 & 1 & 1 & 1 & 1 & $\mathrm{~N}$ & 0 & 0 & 0 \\
\hline 167 & 1 & 1 & 1 & 1 & 1 & 1 & 1 & $0 / 1$ & 0 & 0 \\
\hline 168 & 0 & 0 & 0 & 0 & 0 & 0 & 0 & $0 / 1$ & 0 & 0 \\
\hline 169 & 1 & 1 & l & l & 1 & 1 & 1 & $\mathbf{N}$ & 0 & 0 \\
\hline 170 & 0 & 0 & 0 & 0 & 0 & 0 & 0 & $\mathrm{~N}$ & 0 & 0 \\
\hline 171 & 1 & 1 & 1 & $\mathbf{N}$ & $\mathbf{N}$ & $\mathbf{N}$ & $\mathrm{N}$ & $\mathrm{N}$ & 0 & 0 \\
\hline 172 & 1 & 1 & 1 & 1 & 0 & 0 & 0 & $\mathrm{~N}$ & 0 & 0 \\
\hline 173 & 0 & 0 & 0 & 1 & 1 & 1 & 1 & $\mathbf{N}$ & 0 & 0 \\
\hline 174 & 0 & 0 & 0 & 0 & 1 & 1 & 1 & $\mathbf{N}$ & 0 & 0 \\
\hline 175 & 0 & 0 & 0 & 0 & 0 & 0 & 0 & 0 & 0 & 0 \\
\hline 176 & 0 & 0 & 0 & 0 & 0 & 0 & $0 / 1$ & 0 & 0 & 0 \\
\hline 177 & 1 & 1 & 1 & 1 & 1 & 1 & l & $\mathrm{N}$ & 0 & 0 \\
\hline 178 & 1 & 1 & 1 & 1 & 1 & 1 & l & 0 & 0 & 0 \\
\hline 179 & 1 & 1 & 1 & 1 & 1 & I & I & 0 & 0 & 0 \\
\hline 180 & 0 & 0 & 0 & 1 & 1 & 1 & 1 & 0 & 0 & 0 \\
\hline 181 & 0 & 0 & 0 & 1 & 1 & 1 & 1 & 0 & 0 & 0 \\
\hline 182 & 1 & 1 & 1 & 1 & 1 & 1 & 1 & 0 & 0 & 0 \\
\hline 183 & 0 & 0 & 0 & 0 & I & $?$ & $1 / 0$ & 0 & 0 & 0 \\
\hline 184 & 2 & 2 & 2 & 2 & 2 & 2 & 2 & 0 & 0 & 0 \\
\hline 185 & 0 & 0 & 0 & 0 & 1 & 1 & 1 & $0 / 1$ & 0 & 0 \\
\hline 186 & 1 & 1 & 1 & 1 & 1 & 1 & $1 / N$ & 0 & 0 & 0 \\
\hline 187 & 1 & 1 & 1 & 1 & 1 & 1 & 1 & 1 & 0 & 0 \\
\hline 188 & 2 & 2 & 2 & 2 & 3 & 3 & $3 / \mathrm{N}$ & 1 & 0 & 0 \\
\hline 189 & 1 & 1 & 1 & 1 & 1 & 1 & 1 & 1 & 0 & 0 \\
\hline 190 & I & 1 & I & 1 & 1 & 1 & 1 & 1 & 0 & 0 \\
\hline $19 \mathrm{I}$ & 0 & 0 & 0 & 0 & 0 & 0 & 0 & 0 & 0 & 0 \\
\hline 192 & 0 & 0 & 0 & 0 & 1 & 1 & 1 & 1 & 0 & 0 \\
\hline 193 & 0 & 0 & 0 & 0 & 0 & 0 & 0 & 0 & 0 & 0 \\
\hline 194 & 1 & 1 & 1 & 1 & 1 & 1 & I & 0 & 0 & 0 \\
\hline 195 & 0 & 0 & 0 & 0 & 0 & 0 & $0 / \mathbf{N}$ & 0 & 0 & 1 \\
\hline
\end{tabular}


Table 2-contd.

\begin{tabular}{ccccccccccc}
\hline & $\begin{array}{c}\text { Procyno- } \\
\text { suchus }\end{array}$ & $\begin{array}{c}\text { Thrinax- } \\
\text { odon }\end{array}$ & $\begin{array}{c}\text { Dindem- } \\
\text { odon }\end{array}$ & $\begin{array}{c}\text { Exaeret- } \\
\text { odon }\end{array}$ & $\begin{array}{c}\text { Trityl- } \\
\text { odontidac }\end{array}$ & $\begin{array}{c}\text { Morganuc- } \\
\text { odontidae }\end{array}$ & Mammalia & $\begin{array}{c}\text { Capto- } \\
\text { Testudines }\end{array}$ Araco- \\
rhinidac & scelidia \\
\hline 196 & $\mathrm{~N}$ & $\mathrm{~N}$ & $\mathrm{~N}$ & $\mathrm{~N}$ & $\mathrm{~N}$ & $\mathrm{~N}$ & $\mathrm{~N}$ & 0 & 0 & $\mathrm{~N}$ \\
197 & 0 & 0 & 0 & 0 & 1 & 1 & 1 & $\mathrm{~N} / \mathrm{l}$ & 0 & 0 \\
198 & 0 & 0 & 0 & 0 & 2 & 2 & 2 & 0 & 0 & 0 \\
199 & 0 & 0 & 0 & 0 & 0 & 0 & 0 & $0 / 1$ & 0 & 0 \\
200 & 1 & 1 & 1 & 1 & 1 & $?$ & 1 & 1 & 1 & 1 \\
201 & 0 & 0 & 0 & 0 & 0 & 0 & 0 & 0 & 0 & 0 \\
202 & 0 & 0 & 0 & 0 & 0 & 0 & 0 & 0 & 0 & 1 \\
203 & 0 & 0 & 0 & $?$ & 0 & $?$ & $0 / \mathrm{N}$ & $0 / 1$ & 0 & 0 \\
204 & 0 & 0 & 0 & 0 & 0 & 0 & 0 & $0 / 1$ & 0 & 0 \\
205 & 0 & 0 & 0 & 0 & 0 & 0 & 0 & $0 / 1$ & 0 & 0 \\
206 & 1 & 1 & 1 & 1 & 1 & 1 & 1 & $0 / 1$ & 0 & 0 \\
207 & $\mathrm{I}$ & 1 & 1 & 1 & 1 & 1 & 1 & 1 & 0 & 0 \\
\hline
\end{tabular}

\begin{tabular}{|c|c|c|c|c|c|c|c|c|c|c|}
\hline & $\begin{array}{c}\text { Lepido- } \\
\text { sauromorpha }\end{array}$ & $\begin{array}{l}\text { Rhyncho- } \\
\text { sauria }\end{array}$ & $\begin{array}{l}\text { Trilopho- } \\
\text { saurus }\end{array}$ & $\begin{array}{c}\text { Chorist- } \\
\text { odera }\end{array}$ & $\begin{array}{l}\text { Protoro- } \\
\text { sauria }\end{array}$ & $\begin{array}{l}\text { Protero- } \\
\text { suchidae }\end{array}$ & $\begin{array}{l}\text { Erythro- } \\
\text { suchidae }\end{array}$ & $\begin{array}{c}\text { Protero- } \\
\text { champsidae }\end{array}$ & $\begin{array}{l}\text { Pseudo- } \\
\text { suchia }\end{array}$ & $\begin{array}{c}\text { Ornitho- } \\
\text { suchia }\end{array}$ \\
\hline 1 & 0 & 1 & 1 & l & 1 & 1 & 1 & 1 & 1 & I \\
\hline 2 & $0 / 1$ & I & I & I & 1 & 1 & 1 & 1 & 1 & $1 / 0$ \\
\hline 3 & 0 & 0 & 0 & 0 & 0 & 0 & 0 & 0 & $0 / \mathrm{N}$ & $\mathrm{N}$ \\
\hline 4 & 0 & 0 & 0 & 1 & 1 & 1 & 1 & 1 & 1 & 1 \\
\hline 5 & 0 & 0 & 0 & 0 & 0 & 0 & 0 & 0 & 0 & $0 / 1$ \\
\hline 6 & $1 / 0 / \mathrm{N}$ & 1 & 1 & I & 1 & 1 & 1 & $\mathrm{~N}$ & $1 / 0 / \mathrm{N}$ & $1 / N$ \\
\hline 7 & $0 / \mathrm{N}$ & 0 & ? & 0 & 0 & 0 & 0 & 0 & 0 & $0 / \mathrm{N}$ \\
\hline 8 & 0 & 0 & 0 & 0 & 0 & 0 & 0 & 0 & 0 & 0 \\
\hline 9 & $0 / 1$ & 0 & 0 & 0 & 0 & 0 & 0 & 1 & $0 / 1$ & $0 / 1$ \\
\hline 10 & $0 / 1 / \mathrm{N}$ & 0 & 0 & 0 & 0 & l & 1 & $\mathrm{~N}$ & $1 / \mathrm{N}$ & $1 / \mathrm{N}$ \\
\hline 11 & $0 / \mathrm{N}$ & 0 & 0 & 0 & 0 & 0 & 0 & 0 & 0 & 0 \\
\hline 12 & $0 / 1$ & 0 & $?$ & 0 & 0 & 0 & 0 & 0 & 0 & 0 \\
\hline 13 & $0 / 1$ & 0 & ? & 0 & 0 & 0 & 0 & 0 & 0 & 0 \\
\hline 14 & $0 / 1 / \mathrm{N}$ & 0 & ? & 0 & 0 & 0 & 0 & 0 & 0 & 0 \\
\hline 15 & $1 / 0 / 2$ & $1 / 2$ & 1 & I & 1 & 1 & I & 1 & 1 & $1 / 0$ \\
\hline 16 & $0 / 1$ & $0 / 1$ & 1 & 1 & $0 / 1$ & 0 & 0 & 1 & $1 / 0$ & 1 \\
\hline 17 & $0 / 1 / \mathrm{N}$ & $1 / \mathrm{N}$ & $?$ & $\mathrm{~N}$ & $1 / \mathrm{N}$ & l & 1 & $\mathrm{~N}$ & $\mathrm{~N}$ & $N$ \\
\hline 18 & 0 & 0 & 0 & 0 & 0 & 0 & 0 & 0 & 0 & 0 \\
\hline 19 & $0 / 1$ & 0 & 0 & 0 & 0 & 0 & 0 & 0 & 0 & 0 \\
\hline 20 & $1 / 0$ & 1 & 1 & 1 & 1 & 1 & 1 & 1 & 1 & 1 \\
\hline 21 & 0 & 0 & 0 & 0 & 0 & 0 & 0 & 0 & 0 & 0 \\
\hline 22 & $0 / \mathrm{N}$ & $\mathrm{N}$ & ? & $\mathrm{N}$ & $? / \mathrm{N}$ & 1 & 1 & 1 & 1 & 1 \\
\hline 23 & $0 / 1$ & $0 / 1$ & ? & I & $0 / 1$ & 0 & 1 & 1 & 1 & 1 \\
\hline 24 & $1 / N$ & $1 / N$ & ? & $N$ & 1 & 1 & $\mathrm{~N}$ & $N$ & $N$ & $N$ \\
\hline 25 & $0 / 1$ & 1 & $?$ & 1 & 1 & 1 & 1 & 1 & 1 & 1 \\
\hline 26 & $1 / \mathrm{N}$ & $N$ & $\mathrm{~N}$ & $\mathrm{~N}$ & $\mathrm{~N}$ & $\mathrm{~N}$ & $\mathrm{~N}$ & $\mathrm{~N}$ & $\mathbf{N}$ & $\mathrm{N}$ \\
\hline 27 & 0 & 0 & ? & 0 & 0 & 0 & 0 & 0 & 0 & $0 / 1 / \mathrm{N}$ \\
\hline 28 & 0 & 0 & 0 & 0 & 0 & 0 & 0 & 0 & 0 & 0 \\
\hline 29 & $0 / \mathrm{N} / \mathrm{l}$ & 0 & 0 & 0 & 0 & 0 & 0 & 0 & $0 / 2$ & 0 \\
\hline 30 & 0 & 1 & 0 & 0 & 0 & 0 & 0 & 0 & $0 / 1$ & $0 / 1$ \\
\hline 31 & 0 & 0 & 0 & 0 & 0 & 1 & 1 & 1 & $1 / 0$ & $1 / 0$ \\
\hline 32 & 0 & 0 & 0 & 0 & 0 & 0 & 0 & 0 & 1 & 1 \\
\hline 33 & 1 & 1 & I & I & 1 & 1 & 1 & 1 & 1 & 1 \\
\hline 34 & $0 / 1$ & 1 & 1 & $\mathrm{~N}$ & $1 / 0$ & 1 & I & 1 & 1 & 1 \\
\hline 35 & 1 & 1 & 1 & 1 & 1 & 2 & 2 & 2 & 2 & 2 \\
\hline 36 & 1 & 1 & 1 & 1 & 1 & 2 & 2 & 2 & 2 & 2 \\
\hline 37 & 1 & 1 & 0 & I & 1 & 1 & I & 1 & 1 & 1 \\
\hline 38 & 1 & 1 & 1 & 2 & 1 & 1 & 1 & $1 / 2$ & $1 / 2$ & 1 \\
\hline 39 & $1 / \mathrm{N}$ & 1 & 1 & 1 & 1 & 1 & 1 & 1 & 1 & $1 / \mathrm{N}$ \\
\hline 40 & $\mathrm{~N}$ & $N$ & $\mathrm{~N}$ & $\mathrm{~N}$ & $\mathrm{~N}$ & $\mathrm{~N}$ & $\mathrm{~N}$ & $\mathrm{~N}$ & $\mathrm{~N}$ & $N$ \\
\hline 41 & 1 & 1 & 1 & $\mathrm{~N}$ & 1 & $\mathrm{~N}$ & 2 & $2 / 0$ & $2 / 0 / \mathrm{N}$ & $2 / 0 / \mathrm{N}$ \\
\hline
\end{tabular}


Table 2 contd

\begin{tabular}{|c|c|c|c|c|c|c|c|c|c|c|}
\hline & $\begin{array}{l}\text { Iepido- } \\
\text { sauromorpha }\end{array}$ & $\begin{array}{l}\text { Rhyncho- } \\
\text { sauria }\end{array}$ & $\begin{array}{l}\text { Trilopho- } \\
\text { saurus }\end{array}$ & $\begin{array}{l}\text { Chorist- } \\
\text { odera }\end{array}$ & $\begin{array}{l}\text { Protoro- } \\
\text { sauria }\end{array}$ & $\begin{array}{l}\text { Protero- } \\
\text { suchidae }\end{array}$ & $\begin{array}{l}\text { Erythro- } \\
\text { suchidae }\end{array}$ & $\begin{array}{l}\text { Protero- } \\
\text { champsidae }\end{array}$ & $\begin{array}{l}\text { Pseudo- } \\
\text { suchia }\end{array}$ & $\begin{array}{l}\text { Ornitho- } \\
\text { suchia }\end{array}$ \\
\hline 42 & 0 & 0 & 0 & 0 & 0 & 0 & 0 & 0 & 0 & $0 / 1$ \\
\hline 43 & $0 / 1$ & 0 & 0 & 0 & 0 & 0 & 0 & 0 & 0 & $0 / 1$ \\
\hline 44 & $0 / \mathrm{N} / \mathrm{l}$ & 0 & 0 & 0 & 0 & 0 & 0 & 0 & $0 / 1$ & 0 \\
\hline 45 & 0 & 0 & 0 & 0 & 0 & 0 & 0 & 0 & 0 & 0 \\
\hline 46 & $0 / \mathrm{N}$ & 0 & 0 & 0 & 0 & 0 & 0 & 0 & 0 & 0 \\
\hline 47 & $0 / 1$ & 0 & 0 & 1 & 1 & 1 & 1 & 1 & $1 / N$ & 1 \\
\hline 48 & $0 / 1$ & 0 & I & 1 & 1 & 1 & 1 & 1 & 1 & I \\
\hline 49 & 0 & 0 & 0 & 0 & 0 & 0 & 0 & 0 & 0 & 0 \\
\hline 50 & $0 / 1$ & 1 & 1 & 1 & 1 & 1 & 1 & 1 & 1 & 1 \\
\hline 51 & $0 / \mathrm{N}$ & 0 & 0 & $0 / \mathrm{N}$ & 0 & 0 & 0 & 0 & 0 & 0 \\
\hline 52 & $0 / \mathrm{N}$ & 1 & $\mathrm{~N}$ & $\mathrm{~N}$ & $1 / N$ & 1 & 1 & 1 & 1 & 1 \\
\hline 53 & $0 / \mathbf{N}$ & 0 & 0 & 0 & $0 / \mathrm{N}$ & 0 & 0 & 0 & 0 & 0 \\
\hline 54 & 0 & 0 & 0 & 1 & 0 & 0 & 0 & 0 & 0 & 0 \\
\hline 55 & 0 & 0 & 0 & 0 & 0 & 0 & 0 & 0 & 0 & $0 / 1$ \\
\hline 56 & $1 / \mathrm{N}$ & $\mathrm{N}$ & $?$ & $N$ & $\mathrm{~N}$ & $\mathrm{~N}$ & $N$ & $\mathrm{~N}$ & $\mathrm{~N}$ & $N$ \\
\hline 57 & 2 & 2 & 2 & 2 & 2 & 2 & 2 & 2 & 2 & $2 / 1 / N$ \\
\hline 58 & $0 / 1$ & 0 & ? & 1 & 0 & 0 & 0 & 1 & $0 / 1$ & $0 / 1$ \\
\hline 59 & 0 & 0 & 0 & 0 & 0 & 0 & 0 & 0 & 0 & 0 \\
\hline 60 & $0 / 1$ & 0 & 0 & 0 & 0 & 0 & 0 & 0 & 0 & $0 / 1$ \\
\hline 61 & 0 & 0 & 0 & 0 & 0 & 0 & 0 & 0 & 0 & 0 \\
\hline 62 & 1 & 1 & 1 & 1 & 1 & 1 & 1 & 1 & 1 & 1 \\
\hline 63 & 0 & 0 & 0 & 0 & 0 & 0 & 0 & 0 & 0 & 0 \\
\hline 64 & 0 & 0 & 0 & 0 & 0 & 0 & 0 & 0 & 0 & 0 \\
\hline 65 & $0 / \mathrm{N}$ & 0 & 0 & 0 & 0 & 0 & $?$ & $?$ & $0 / \mathrm{N}$ & $0 / \mathrm{N}$ \\
\hline 66 & 0 & 0 & 0 & 0 & 0 & 0 & ? & $?$ & $0 / \mathrm{N}$ & $0 / \mathrm{N}$ \\
\hline 67 & 0 & 0 & 0 & 0 & 0 & 0 & 0 & 0 & $0 / \mathrm{N}$ & $0 / \mathrm{N}$ \\
\hline 68 & $0 / 1$ & 1 & I & 1 & 1 & 1 & 1 & 1 & 1 & 1 \\
\hline 69 & 1 & 1 & 1 & 1 & 1 & $?$ & $?$ & ? & 1 & 1 \\
\hline 70 & $0 / 1 / 2$ & $1 / 0$ & 2 & 1 & $1 / 0$ & 1 & 1 & 1 & $1 / 0$ & $1 / 0 / 2$ \\
\hline 71 & 0 & 0 & 0 & 0 & 0 & 1 & 1 & ? & 1 & 1 \\
\hline 72 & 0 & 0 & 0 & 0 & 0 & 0 & $?$ & ? & 0 & 0 \\
\hline 73 & 0 & 0 & 0 & 0 & 0 & 0 & 0 & 0 & 0 & 0 \\
\hline 74 & 1 & 1 & 1 & I & I & I & I & 1 & 1 & 1 \\
\hline 75 & 0 & 0 & 0 & 0 & 0 & 0 & 0 & 0 & 0 & 0 \\
\hline 76 & 0 & 0 & 0 & 0 & 0 & 0 & 0 & 0 & 0 & 0 \\
\hline 77 & 0 & 0 & 0 & 0 & 0 & 0 & 1 & 1 & 1 & 1 \\
\hline 78 & 0 & 1 & 1 & 1 & 1 & 1 & 1 & 1 & 1 & $1 / 0$ \\
\hline 79 & 0 & 0 & 0 & 0 & 0 & 0 & 0 & 0 & 0 & 0 \\
\hline 80 & $0 / 1$ & 0 & 0 & 0 & 0 & 0 & 0 & 0 & 1 & 1 \\
\hline 81 & 0 & 0 & 0 & 0 & 0 & 0 & 0 & 0 & 0 & 0 \\
\hline 82 & 1 & l & $?$ & 1 & 1 & 1 & 1 & 1 & 1 & 1 \\
\hline 83 & 1 & 1 & $?$ & 1 & 1 & 1 & 1 & 1 & 1 & 1 \\
\hline 84 & 0 & 0 & 0 & 0 & 0 & 0 & ? & 0 & 0 & 0 \\
\hline 85 & 0 & 0 & 0 & 0 & 0 & 0 & 0 & 0 & 0 & 0 \\
\hline 86 & 0 & 0 & 0 & 0 & 0 & 0 & 0 & 0 & 0 & 0 \\
\hline 87 & 0 & 0 & 0 & 0 & 0 & 0 & 1 & 1 & 1 & $1 / 0$ \\
\hline 88 & 0 & 0 & 0 & 0 & 0 & 0 & 0 & 0 & 0 & 0 \\
\hline 89 & 0 & 0 & 0 & 0 & 0 & 0 & 0 & 0 & 0 & 0 \\
\hline 90 & 0 & 0 & 0 & 0 & 0 & 0 & 0 & 0 & 0 & 0 \\
\hline 91 & 0 & 0 & 0 & 0 & 0 & 0 & 0 & 0 & 0 & 0 \\
\hline 92 & 0 & 0 & 0 & 0 & 0 & 0 & 0 & 0 & 0 & 0 \\
\hline 93 & 0 & 0 & 0 & 0 & 0 & 0 & 0 & 0 & 0 & 0 \\
\hline 94 & 0 & 0 & 0 & 0 & 0 & 0 & 0 & 0 & 0 & 0 \\
\hline 95 & 0 & 0 & 0 & 0 & 0 & 0 & 0 & 0 & 0 & $0 / 1$ \\
\hline 96 & 0 & 0 & 0 & 0 & 0 & 0 & 0 & 0 & 0 & 0 \\
\hline 97 & 0 & 0 & 0 & 0 & 0 & 0 & 0 & 0 & 0 & $0 / 1$ \\
\hline 98 & 0 & 0 & 0 & 0 & 0 & 0 & 0 & 0 & 0 & 0 \\
\hline 99 & 0 & 0 & 0 & 0 & 0 & 0 & 0 & 0 & 0 & 0 \\
\hline
\end{tabular}


Table 2 -contd.

\begin{tabular}{|c|c|c|c|c|c|c|c|c|c|c|}
\hline & $\begin{array}{c}\text { Lepido- } \\
\text { sauromorpha }\end{array}$ & $\begin{array}{c}\text { Rhyncho- } \\
\text { sauria }\end{array}$ & $\begin{array}{l}\text { Trilopho- } \\
\text { saurus }\end{array}$ & $\begin{array}{c}\text { Chorist- } \\
\text { odera }\end{array}$ & $\begin{array}{l}\text { Protoro- } \\
\text { sauria }\end{array}$ & $\begin{array}{l}\text { Protero- } \\
\text { suchidae }\end{array}$ & $\begin{array}{l}\text { Erythro- } \\
\text { suchidae }\end{array}$ & $\begin{array}{c}\text { Protero- } \\
\text { champsidae }\end{array}$ & $\begin{array}{l}\text { Pseudo- } \\
\text { suchia }\end{array}$ & $\begin{array}{l}\text { Ornitho- } \\
\text { suchia }\end{array}$ \\
\hline 100 & 1 & 1 & 1 & 1 & 1 & 1 & 1 & 1 & 1 & $1 / \mathbf{N}$ \\
\hline I01 & 0 & 0 & 0 & 0 & 0 & 0 & 0 & 0 & 0 & 0 \\
\hline 102 & 0 & 0 & 0 & 0 & 0 & 0 & 0 & 0 & 0 & 0 \\
\hline 103 & 0 & 0 & 0 & 0 & 0 & 0 & 0 & 0 & 0 & 0 \\
\hline 104 & 1 & 1 & 1 & 1 & 1 & 1 & 1 & 1 & 1 & 1 \\
\hline 105 & 0 & 0 & 0 & 0 & 0 & 0 & 0 & 0 & 0 & 0 \\
\hline 106 & 0 & 0 & 0 & 0 & 0 & 0 & I & I & 1 & $\mathrm{I} / \mathrm{N}$ \\
\hline 107 & 0 & 0 & 0 & 0 & 0 & 0 & 1 & 1 & 1 & $1 / \mathrm{N}$ \\
\hline 108 & $0 / 1$ & 0 & 0 & 0 & 0 & 1 & 1 & 1 & $1 / 0$ & $1 / 0 / \mathrm{N}$ \\
\hline 109 & 0 & 0 & 0 & 0 & 1 & 1 & 1 & 1 & 1 & $1 / N$ \\
\hline 110 & 0 & 0 & 0 & 0 & 0 & 1 & 1 & 1 & 1 & $1 / N$ \\
\hline 111 & 1 & 1 & 1 & 1 & 1 & 1 & 1 & 1 & 1 & $1 / N$ \\
\hline 112 & 0 & 0 & 0 & 0 & 0 & 0 & 0 & 0 & 0 & 0 \\
\hline 113 & $\mathbf{N}$ & $\mathbf{N}$ & $\mathbf{N}$ & $\mathrm{N}$ & $N$ & $\mathbf{N}$ & $\mathrm{N}$ & $\mathrm{N}$ & $\mathrm{N}$ & $\mathrm{N}$ \\
\hline 114 & $\mathbf{N}$ & $\mathrm{N}$ & $\mathrm{N}$ & $\mathrm{N}$ & $\mathrm{N}$ & $\mathbf{N}$ & $\mathrm{N}$ & $\mathrm{N}$ & $\mathbf{N}$ & $\mathrm{N}$ \\
\hline 115 & $0 / 1$ & 0 & 1 & 0 & 0 & 0 & 0 & 0 & 0 & $0 / \mathbf{l} / \mathbf{N}$ \\
\hline 116 & $\mathrm{~N}$ & $\mathbf{N}$ & $\mathrm{N}$ & $\mathbf{N}$ & $N$ & $\mathbf{N}$ & $\mathrm{N}$ & $\mathrm{N}$ & $\mathbf{N}$ & $\mathrm{N}$ \\
\hline 117 & $0 / 1$ & 0 & 1 & 0 & 0 & 0 & 0 & 0 & 0 & $0 / \mathrm{N}$ \\
\hline 118 & 0 & 0 & 0 & 0 & 0 & 0 & 0 & 0 & 0 & $0 / \mathrm{N}$ \\
\hline 119 & 0 & 0 & 0 & 0 & 0 & 0 & 1 & 1 & 1 & $1 / \mathrm{N} / 0$ \\
\hline 120 & $0 / 1$ & $0 / 1$ & 1 & 0 & $0 / 1$ & 0 & 0 & 0 & $0 / 1$ & $0 / 1$ \\
\hline 121 & $0 / 1$ & 0 & 1 & 0 & 0 & 0 & 1 & 0 & $0 / 1$ & $0 / 1$ \\
\hline 122 & 1 & 1 & 1 & 1 & 1 & 1 & 1 & 1 & 1 & $1 / \mathbf{N}$ \\
\hline 123 & $0 / 1$ & $0 / 1$ & 1 & 0 & 0 & 0 & I & 1 & 1 & 1 \\
\hline 124 & $0 / 1$ & 1 & 1 & 1 & 1 & I & 1 & 1 & 1 & 1 \\
\hline 125 & 0 & 0 & 0 & 0 & 0 & 0 & 0 & 0 & 0 & 0 \\
\hline 126 & 0 & 0 & 0 & 0 & 0 & 0 & 0 & 0 & 1 & 1 \\
\hline 127 & $0 / 1$ & 0 & 0 & 1 & 0 & 0 & 1 & 1 & 1 & $0 / 1$ \\
\hline 128 & $0 / 1$ & 0 & 0 & 1 & 0 & 0 & 1 & 1 & I & $0 / \mathrm{L}$ \\
\hline 129 & 0 & 0 & 0 & 0 & 0 & 0 & 0 & 1 & 1 & $0 / 1$ \\
\hline 130 & 0 & 0 & 0 & 0 & 0 & 0 & 0 & 0 & 0 & 0 \\
\hline 131 & 1 & 1 & 1 & 1 & 1 & $?$ & $?$ & 1 & 1 & 1 \\
\hline 132 & 1 & 1 & 1 & 1 & 1 & 1 & $?$ & 1 & 1 & 1 \\
\hline 133 & $0 / 1$ & 0 & 0 & 0 & 0 & 0 & 0 & 0 & 0 & $0 / 1$ \\
\hline 134 & 0 & 0 & 0 & 0 & 0 & 0 & 0 & 0 & 0 & 0 \\
\hline 135 & 0 & 0 & 0 & 0 & 0 & 0 & 0 & 0 & 0 & 0 \\
\hline 136 & 0 & 0 & 0 & 0 & 0 & 0 & ? & 0 & 0 & $0 / 1$ \\
\hline 137 & 0 & 0 & 0 & 0 & 0 & 0 & $?$ & 0 & 0 & 0 \\
\hline 138 & 0 & 0 & 0 & 0 & 0 & 0 & 0 & 0 & 0 & 0 \\
\hline 139 & 1 & 1 & 1 & 1 & 1 & 1 & 1 & 1 & 1 & 1 \\
\hline 140 & 0 & 0 & 0 & 0 & 0 & 0 & 0 & 0 & 0 & 0 \\
\hline 141 & 0 & 0 & 0 & $0 / 1$ & 0 & 0 & 0 & 0 & $0 / 1$ & $0 / 1$ \\
\hline 142 & 1 & 1 & 1 & 1 & 1 & 1 & 1 & 1 & 1 & 1 \\
\hline 143 & 0 & 0 & 1 & 1 & 1 & 1 & 1 & 1 & l & I \\
\hline 144 & 0 & 0 & 0 & 0 & 0 & 0 & 0 & 0 & 1 & 1 \\
\hline 145 & 0 & 0 & 0 & 0 & 0 & 0 & 0 & 0 & 0 & 0 \\
\hline 146 & 0 & 0 & 0 & 0 & 0 & 0 & 1 & 1 & 1 & 1 \\
\hline 147 & 1 & 1 & 1 & 1 & 1 & 1 & 1 & 1 & 1 & 1 \\
\hline 148 & 0 & 0 & 0 & 0 & 0 & 0 & 0 & 0 & 0 & 0 \\
\hline 149 & 1 & 1 & 1 & 1 & 1 & 1 & 1 & 1 & 1 & 1 \\
\hline 150 & 0 & 0 & 0 & 0 & 0 & 0 & 0 & 0 & 0 & 0 \\
\hline 151 & $0 / 1$ & 1 & 1 & 1 & 1 & 1 & ? & 1 & 1 & 1 \\
\hline 152 & $0 / 1$ & $\mathrm{~N}$ & $\mathrm{~N}$ & $\mathrm{~N}$ & $\mathrm{~N}$ & $\mathrm{~N}$ & $\dot{N}$ & $\mathrm{~N}$ & $\mathrm{~N}$ & $\mathrm{~N}$ \\
\hline 153 & $1 / N$ & 1 & 1 & 1 & 1 & 1 & $?$ & $?$ & $1 / \mathbf{N}$ & $1 / \mathrm{N}$ \\
\hline 154 & $1 / 0 / \mathrm{N}$ & 1 & 1 & 1 & 1 & 1 & 1 & ? & 1 & $\mathrm{l} / \mathrm{N}$ \\
\hline 155 & $0 / 1 / N$ & 0 & 0 & 0 & 0 & 0 & 0 & ? & 1 & $1 / \mathrm{N}$ \\
\hline 156 & $0 / 1 / N$ & 0 & 0 & 0 & 0 & 0 & 0 & ? & 0 & $0 / \mathrm{N}$ \\
\hline 157 & $0 / 1$ & $?$ & $?$ & 0 & 0 & 0 & $?$ & $?$ & 0 & $0 / 1$ \\
\hline 158 & 0 & 0 & 0 & 0 & 0 & 0 & 0 & 0 & 0 & 0 \\
\hline
\end{tabular}


Table 2 contd.

\begin{tabular}{|c|c|c|c|c|c|c|c|c|c|c|}
\hline & $\begin{array}{l}\text { Lepido- } \\
\text { sauromorpha }\end{array}$ & $\begin{array}{l}\text { Rhyncho- } \\
\text { sauria }\end{array}$ & $\begin{array}{l}\text { Tribopho- } \\
\text { saurus }\end{array}$ & $\begin{array}{l}\text { Chorist- } \\
\text { odera }\end{array}$ & $\begin{array}{l}\text { Protoro- } \\
\text { sauria }\end{array}$ & $\begin{array}{l}\text { Protero- } \\
\text { suchidae }\end{array}$ & $\begin{array}{l}\text { Erythro- } \\
\text { suchidae }\end{array}$ & $\begin{array}{c}\text { Protero- } \\
\text { champsidae }\end{array}$ & $\begin{array}{l}\text { Pseudo- } \\
\text { suchia }\end{array}$ & $\begin{array}{l}\text { Ornitho- } \\
\text { suchia }\end{array}$ \\
\hline 159 & 1 & 1 & 1 & 1 & 1 & 1 & 1 & 1 & 1 & 1 \\
\hline 160 & 0 & 0 & 0 & 0 & 0 & 0 & 0 & 1 & 1 & 1 \\
\hline 161 & 0 & 0 & 0 & 0 & 0 & 0 & 0 & 0 & 0 & 0 \\
\hline 162 & 0 & 0 & 0 & 0 & 0 & 0 & 1 & 1 & 1 & 1 \\
\hline 163 & 1 & 0 & 0 & 0 & 0 & 0 & $\mathbf{N}$ & $\mathrm{N}$ & $N$ & $\mathrm{~N}$ \\
\hline 164 & $0 / 1$ & 1 & 1 & 1 & 1 & 1 & 1 & 1 & 1 & 1 \\
\hline 165 & 0 & 0 & 0 & 0 & 0 & 0 & 0 & 0 & 0 & 0 \\
\hline 166 & $0 / \mathrm{N}$ & 0 & 0 & 0 & 0 & 0 & 0 & 0 & $0 / \mathrm{N}$ & $0 / \mathrm{N}$ \\
\hline 167 & 0 & 0 & 0 & I & 0 & 0 & 0 & 1 & 1 & 1 \\
\hline 168 & 0 & 0 & 0 & 0 & 0 & 0 & 0 & 1 & 1 & 1 \\
\hline 169 & 1 & 1 & 1 & I & 1 & 1 & 1 & 1 & 1 & 1 \\
\hline 170 & 1 & 1 & 1 & 1 & l & 1 & 1 & 1 & 1 & 1 \\
\hline 171 & $1 / 0$ & 1 & 1 & 1 & 1 & 1 & 1 & 1 & 1 & 1 \\
\hline 172 & 0 & 0 & 0 & 0 & 0 & 0 & 0 & 0 & $0 / 1$ & $0 / 1$ \\
\hline 173 & 0 & 0 & 0 & 0 & 0 & 0 & 0 & 0 & 0 & 0 \\
\hline 174 & 0 & 0 & 0 & 0 & 0 & 0 & 0 & 0 & 0 & 0 \\
\hline 175 & $1 / 0$ & 1 & 1 & 1 & 1 & 1 & 1 & 1 & 1 & 1 \\
\hline 176 & 1 & 1 & 1 & 1 & 1 & 1 & 1 & 1 & 1 & 1 \\
\hline 177 & $1 / N$ & 1 & 1 & 1 & 1 & 1 & 1 & 1 & 1 & 1 \\
\hline 178 & $0 / \mathrm{N}$ & 0 & 0 & 0 & 0 & 0 & 0 & 0 & $0 / 1$ & $0 / 1$ \\
\hline 179 & $0 / \mathrm{N}$ & 0 & 0 & 0 & 0 & 0 & 0 & 0 & $0 / 1$ & $0 / 1$ \\
\hline 180 & $0 / \mathrm{N}$ & 0 & 0 & 0 & 0 & 0 & 0 & 0 & 0 & 0 \\
\hline 181 & $0 / \mathrm{N}$ & 0 & 0 & 0 & 0 & 0 & 0 & 0 & 0 & 0 \\
\hline 182 & 0 & 0 & 0 & 0 & 0 & 0 & 0 & 0 & $0 / \mathrm{N}$ & $0 / \mathrm{N}$ \\
\hline 183 & 0 & 0 & 0 & 0 & 0 & 0 & 0 & 0 & 0 & 0 \\
\hline 184 & 1 & 1 & 1 & 1 & 1 & 1 & l & 2 & 2 & 2 \\
\hline 185 & 0 & 0 & 0 & 0 & 0 & 0 & 0 & $N$ & $\mathrm{~N}$ & $\mathrm{~N}$ \\
\hline 186 & 0 & 0 & 0 & 0 & 0 & 0 & 0 & 1 & 1 & 1 \\
\hline 187 & 0 & 0 & 0 & 0 & 0 & 0 & 0 & I & 1 & 1 \\
\hline 188 & 0 & 0 & 0 & 0 & 0 & 0 & 0 & 0 & $0 / 1$ & $0 / 1$ \\
\hline 189 & 1 & 1 & 1 & 1 & 1 & 1 & 1 & I & 1 & 1 \\
\hline 190 & $i$ & 1 & 1 & $i$ & 1 & 1 & 1 & 1 & 1 & 1 \\
\hline 191 & 0 & 0 & 0 & 0 & 0 & 0 & 0 & 1 & 1 & $1 / 0$ \\
\hline 192 & 0 & 0 & 0 & 0 & 0 & 0 & 0 & 0 & 0 & 0 \\
\hline 193 & 0 & 0 & 0 & 0 & 0 & 1 & 1 & 1 & 1 & $1 / 0$ \\
\hline 194 & $0 / \mathrm{N}$ & 0 & 0 & 0 & 0 & 0 & 0 & 0 & 0 & 0 \\
\hline 195 & $1 / N$ & $\mathrm{~N}$ & $\mathrm{~N}$ & $\mathrm{~N}$ & $\mathrm{~N}$ & $\mathrm{~N}$ & $\mathrm{~N}$ & $\mathrm{~N}$ & $N$ & $\mathrm{~N}$ \\
\hline 196 & $N$ & 1 & 1 & 1 & 1 & 1 & 1 & 1 & 1 & 1 \\
\hline 197 & 0 & 0 & 0 & 0 & 0 & 0 & $?$ & ? & $\mathrm{N}$ & $N$ \\
\hline 198 & 0 & 1 & 1 & 1 & 1 & I & $?$ & ? & 2 & 2 \\
\hline 199 & $0 / 1$ & 0 & 0 & 1 & 0 & 0 & 1 & 1 & 1 & 1 \\
\hline 200 & 1 & 1 & 1 & ? & 1 & 1 & $?$ & $?$ & 1 & 1 \\
\hline 201 & 1 & 1 & 1 & 1 & 1 & 1 & 1 & 1 & 1 & 1 \\
\hline 202 & 1 & 1 & 1 & 1 & 1 & 1 & $?$ & J & 1 & $1 / 0$ \\
\hline 203 & 0 & 0 & 0 & 0 & 0 & 0 & 1 & 1 & 1 & 1 \\
\hline 204 & 1 & 1 & 1 & 1 & 1 & 1 & 1 & 1 & 1 & $1 / \mathrm{N}$ \\
\hline 205 & $0 / 1$ & 1 & 1 & I & 1 & 1 & 1 & 1 & 1 & $1 / \mathrm{N}$ \\
\hline 206 & $0 / 1$ & $i$ & 1 & 1 & 1 & l & ? & 1 & 1 & 1 \\
\hline 207 & $0 / 1$ & 0 & 0 & $\mathrm{~N}$ & 0 & 0 & $?$ & $N$ & $0 / \mathrm{N}$ & $0 / \mathrm{N}$ \\
\hline
\end{tabular}

$\mathrm{N}=$ character not applicable.

? = character not preserved.

111. Caniniform maxillary tooth. Present $(0)$ or absent (1).

112. Canine root. Not swollen (0) or expanded into and constructing choana (1).

113. Maxillary precanines. More (0), or fewer (1), than 4.

114. Length of canine teeth relative to postcanines. No more than twice as long $(0)$ or at least three times as long (1). 
115. Postcanines. Unicuspid (0) or multicuspid (1).

116. Upper postcanines. 13 or more $(0)$ or 12 or fewer $(1)$.

117. Cheek teeth orientation. Anteroposterior (0), or transverse (1).

118. Cheek teeth roots. Single (0) or at least two (1).

119. Dentary teeth. Subequal in size anteriorly $(0)$ or with an enlarged tooth within a few teeth from symphysis (1).

120. Vomerine teeth. Present (0) or absent (1).

121. Palatine teeth. Present $(0)$ or absent (1).

122. Eclopterygoid teeth. Present $(0)$ or absent (1).

123. Teeth on transwerse process of pterygoid. Present $(0)$ or absent (1).

\section{Axial Skeleton:}

124. Vertebrae. Notochordal (0), or non-notochordal (1), in adult.

125. Vertebral anapophyses. Absent $(0)$ or present (1).

126. Apex of neutral spine. Narrow (0), or expanded (1), in dorsal vicw (viz. "spine tables" present).

127. Intercentra. Present (0), or absent (1), from anterior dorsals.

128. Intercentra. Present $(0)$, or absent (1), from all postcervical trunk vertebrae.

129. Intercentra. Present (0), or absent (1), from postaxial cervicals.

130. Intercentra 1 and 2 . In contact ventrally $(0)$, or separated by atlas centrum (1), in adult.

131. Second intercentrum. Unfused (0), or fused (1), to axis in adult.

132. Second intercentrum. Narrowly $(0)$, or broadly (1), participating in atlanto-axial joint.

133. Odontoid. Separate from axis throughout ontogeny $(0)$ or fused in adult (1).

134. Anterior surface of atlanto-pleurocentrum. Flat $(0)$, or with discrete, finger-like projection anterodorsally (1) (viz. dens present).

135. Atlas arch-intercentrum. In contact $(0)$ or separated (1).

136. Allantal epipophysis. Present (0) or absent (1).

137. Allanto-axial zygapophyses-intervertebral foramen. Prominent and small, (0), or small and large, (1), respectively.

138. Axis centrum. Tall $(0)$ or depressed (1).

139. Cervical centra. Rounded (0), or keeled (1), ventrally.

140. Lumbar verlebrae faces. Perpendicular to notochordal axis (0) or inclined (1).

141. Sacral vertebrae. Two or less $(0)$ or at least three (1).

142. Sacral ribs. Point ventrally $(0)$ or laterally (1).

143. Cervical ribs. Without anterior process and extending posterolaterally $(0)$, or plowshare-shaped, with rib shafts parallel to centra (1).

144. Parasagillal osteoderm rowes. Absent (0) or present (1).

\section{Pectoral Girdle and Forelimb:}

145. Scapula. Flat (0) or concave (1) (viz. infraspinous process present).

146. Scapula. Broad (0) or narrow (1) above glenoid.

147. Supraglenoid buttress. Present (0) or absent (1).

148. Coracoid ossification(s). One (0) or two (1).

149. Postcoracoid cartilage. Present (0) or absent (1).

150. Precoracoid and postcoracoid in glenoid. Present (0) or postcoracoid only (1).

151. Cleithrum. Present (0) or absent (1). 
152. Cleithrum-clavicle. In contact (0) or not (1).

153. Clavicles. Broad (0), or narrow (1), medially.

154. Interclavicle. Rhomboidal (0), or T-shaped (1), anteriorly.

155. Interclavicle lateral processes. Long $(0)$ or short (1).

156. Intercavicle posterior process. Long (0) or short (1).

157. Ossified sternum. Absent (0) or present (1).

158. Sternebrae. Absent (0) or present (1).

159. Limbs. Short and stout (0) or long and slender (1).

160. Humerus. With prominent $(0)$, or reduced (1), epicondyles.

161. Humeral head. Convex articular surface extends broadly across head $(0)$ or articular surface bulbous and inflected (1).

162. Ectepicondylar groove. Present (0) or absent (1).

163. Ectepicondylar groove. Open (0) or enclosed (1) (viz. ectepicondylar foramen present).

164. Entepicondylar foramen. Present (0) or absent (1).

165. Radius width relative to ulna. Radius equal to or narrower (0), or broader (1), than ulna distally.

166. Manual intermedium size relative to centrale. Larger (0) or smaller (1).

167. Longest digit in hand and foot. IV $(0)$ or III (1).

168. Inner two digits of hands and feet. Less $(0)$, or more (1), robust than outer two.

\section{Pelvic Girdle and Hindlimb:}

169. Ilium dorsal groove. Present (0) or absent (1).

170. Ilium posterior process. Short $(0)$ or extends to posterior limit of ischium (1).

171. Ilium. Narrow and posterodorsally oriented $(0)$ or expanded anteriorly and dorsally and with horizontal dorsal margin (1).

172. Ilium. Less than (0), or more than (1), twice height of acetabulum.

173. Ilium. Convex (0), or incised (1), dorsal border.

174. Ilium. Cross-section narrow (0) or triangular (1).

175. Acetabulum. Ilium, ischium and pubis contribute nearly equally to acetabulum $(0)$ or ilium comprises $80 \%-85 \%$ of acetabulum (1).

176. Cotyloid notch. Open (0), or closed, so that a tony ridge completely encloses acetabulum (1).

177. Acetabulum. Oval (0), or circular (1), in outline.

178. Acetabulum. Shallow (0) or deep (1).

179. Supracetabular butiress. Absent (0) or present (1).

180. Acetabulum. Lateral (0), or posterior (1), to sacrum.

181. Pubis-ischium. Diverge from one another below acetabulum (0) or both rotated backwards to lie below rear of acetabulum (1).

182. Obturator foramen. In pubis $(0)$ or between pubis and ischium (1).

183. Epipubic bones. Absent (0) or present (1).

184. Intertrochanteric fossa. Prominent (0), reduced (1) or absent (2).

185. Internal trochanter. On ventral $(0)$, or lateral (1), surface of femur (viz. lesser trochanter present).

186. Femoral head. Terminal $(0)$ or inflected medially $(1)$.

187. Femoral condyles. Prominent $(0)$ or not projecting markedly beyond shaft (1).

188. Femoral head. Elongate (0), subspherical (1), oblong and spherical (2), or protuberant and with constricted neck (3). 
189. Anterior (or medial) femoral condyle relative to posterior (or lateral) condyle. Larger and extends further distally (0) or smaller and of subequal extent distally (1).

190. Femur ventral ridge system. Prominent (0) or feeble (1).

191. Femoral shaft. Weak (0) or prominent (1) sigmoidal curve.

192. Greater trochanter. Confluent with femoral head $(0)$ or separated from articular surface by incisure (1).

193. Raised fourth trachanter. Absent (0) or present (1).

194. Tibia. Articulates on distal ( 0 ) or dorsal (anterior) surface of astragalus (1).

195. Tibio-astragalar joint. Flat, simple joint $(0)$ or ridge (tibia) and groove (astragalus) (1).

196. Tibio-astragalar joint. Flat, simple joint (0) or convex (tibia) and concave (astragalus) (1).

197. Astragalar sulcus. Open (0), or closed (1), posteriorly.

198. Calcaneal tuber. Absent (0), or present and laterally (1), or posteriorly (2), directed.

199. Lateral centrale. Separate from (0), or fused to (1), astragalus in adult.

200. Pedal centrale. Present $(0)$ or absent (1).

201. Proximal tarsals and carpals. Large (0) or small (1).

202. Foot. Short and broad $(0)$ or long and slender (1).

203. Distal tarsal I. Present (0) or absent (1).

204. Fifth metatarsal. Long and slender (0) or short and broad-based (1).

205. Fifth metatarsal. Straight (0), or hooked (1), proximally.

206. Fifth distal tarsal. Present (0) or absent (1).

207. Pedal phalangeal formula. 2-3-4-5-4 (0) or 2-3-4-(4 or 3$)-3$ (1).

\section{Results and Discussion}

\section{The Hy Pothesis}

An experimental approach was used to elucidate the role of fossils in phylogenetic inference. Two taxon/character matrices were employed. One consisted of the five major groups of extant amniotes, and it is referred to as the Recent Data Set (Table 1). The other is termed the Combined Data Set, because it was composed of the five extant plus 24 extinct amniote taxa (Table 2 and the soft anatomy portion of Table 1). Fossil members of the five extant taxa were also surveyed in the latter data set, but not in the former. Only extant outgroups were used to polarize characters in the Recent Data Set, whercas both extant and extinct outgroups were employed in inferring the ancestral condition in the Combined Data Set.

The initial proposition tested was that adding fossils will not lead to a different and better supported phylogenetic hypothesis than the one based only on evidence from Recent amniotes. $A$ single, best-fitting branching pattern was obtained from the Recent Data Set (Fig. 2; length $(L)=175$, consistency index $(c)=0 \cdot 674$ ). Identical most parsimonious topologies resulted from the analyses of the soft and the hard anatomy subsets of characters $(L=108, c=0.694$, and $L=67, c=0.642$, respectively). This is the hypothesis of relationship we refer to as the Recent tree (Fig. 2). It resembles Gardiner's hypothesis (Fig. 1) in that it recovers his Euamniota and Thecodontia.

Analysis of the Combined Data Set also produced a single, best-fitting tree (our hypothesis, Fig. $3 ; L=461, c=0 \cdot 642$ ). The placement of turtles and lepidosaurs are reversed in Figs 2 and 3, and the two trees also differ in the position of mammals. The 
classification (lepidosaurs (turtles (mammals (crocodiles, birds)))) summarizes the Recent hypothesis (Fig. 2), whereas (mammals (turtles (lepidosaurs (crocodiles, birds)))) represents ours (Fig. 3). Thus, we conclude that adding fossils can overturn a theory of relationships based only on evidence from Recent amniotes.

Although our hypothesis (Fig. 3) is the most parsimonious summary of the evidence, we recognize that it is incomplete. We considered most of the well-known taxa and characters, but the length of this manuscript forced us to omit some of both. Some taxa are too poorly known (e.g., Eothyris, Weigeltisaurus), while others are too derived (e.g., ichthyosaurs). Adding such taxa is not expected to change the relative positions of the amniotes we considered (Table 2 and Fig. 3). In fact, when several extinct taxa, Mesosauridae, Millerettidae, Paleothyris, Pareiasauria, and Procolophonia, were included (see Gauthicr et al., 1988b), the resulting cladogram was consistent with our phylogenetic hypothesis (Fig. 3).

We also deleted, or modified, a few characters whose complexity and variability thwarted our attempts to describe and score them across all amniotes and the outgroups. The "sloping occiput", so distinctive of early synapsids, proved to be one of these characters. Daunted by the morphologic variation in the occipital region of Tetrapoda, we elected to replace this traditional character with the more easily recognized and scored "position of the occiput with respect to the suspensorium". The broader outlines of amniote phylogeny that concern us here are not likely to be seriously altered by missing data, regardless of whether it results from non-preservation or from rapidly evolving characters (see below).

\section{AMNIOTE TAXONOMY}

'The currently accepted taxonomy of Amniota (e.g., Romer, 1956), in which all taxa, other than mammals and extant ornithosuchians (i.e., birds; Gauthier, 1986), have been termed "Reptilia", is incongruent with our phylogenetic hypothesis (Fig. 3). We accept Henning's (1966) thesis that there should be a single, coherent system of classification based on propinquity of descent. Accordingly, we adopt a monophyletic taxonomy for the more inclusive categories of extant amniotes, in accordance with the conventions discussed by Gauthier et al. (1988a):

\section{Amniota \\ Mammalia \\ Reptilia \\ Chelonia \\ Sauria \\ Lepidosauria \\ Archosauria}

These taxa can be properly defined. Amniota comprises the most recent common ancestor of mammals and reptiles, and all its descendants. Mammalia is the most recent common ancestor of monotremes and therians and all lineages evolved therefrom. The formerly paraphyletic Reptilia, a name whose meaning has changed more than any other in this taxonomy, with the possible exception of Sauria, is restricted to the most recent common ancestor of turtles and saurians, and all its descendants. Chelonia is limited to the immediate common ancestor of pleurodires and cryptodires, and all forms evolved therefrom. Sauria designates the group containing the most recent common 
ancestor of archosaurs and lepidosaurs, and all its descendants, Lepidosauria includes the most recent common ancestor of Sphenodon and squamates, and all its descendants, and Archosauria is restricted to the most recent common ancestor of crocodiles and birds, and all lineages descended therefrom. Nomenclature for additional clades is listed in Appendix A (see also Fig. 3). Diagnoses for some of the major groups of extant amniotes, namely Reptilia, Sauria, Archosauria, and Mammalia, are provided in Appendix B.

Less familiar names have been applied to these extant groups and one or more of their extinct sister lineages (Fig. 3). For example, Huene's (1946) Archosauromorpha, a junior synonym of Cope's (1869) Archosauria, has been used for archosaurs and all other saurians that are closer to archosaurs (s.s.) than they are to lepidosaurs (s.s.). (The present taxonomic sense of the name Archosauromorpha was first used publicly by Gauthier at the 1982 annual meeting of the American Society of Zoologists. Archosauromorpha was used subsequently by Benton (1983, 1984, 1985), who independently came to the conclusion that there are two major groups of saurians (=neodiapsids of Benton, 1985).)

We have purposely associated the most familiar proper names with sister lineages containing extant taxa. We believe employing such a convention will help to stabilize the meaning of the best known names. In general, there should be a larger number of synapomorphies diagnostic of extant sister groups, because some kinds of evidence will not be preserved in fossils. For example, it is common knowledge that hirsute organisms are mammals. However, many biologists have probably never heard of Morganucodontidae, an extinct taxon that paleontologists typically include within Mammalia. In our view, Morganucodontidae is not a mammal (Fig. 3), because it does not possess the skeletal attributes hypothesized for the most recent common ancestor of the group Monotremata + Theria (Appendix 3). In addition, we cannot be sure that Morganucodontidae possessed the unfossilizable attributes diagnostic of Mammalia, such as hair, mammary glands, four-chambered heart, etc.

Our convention would appear to identify the same assemblages as Hennig's (1981: 29) "*groups" concept (= crown groups of Jefferies (1979)); however, our motives for recognizing such groupings are different. We emphasize nomenclatural stability of commonly used names. Hennig, on the other hand, distinguished * group from stem group, the former consisting of Recent taxa, the latter made up of fossils, because he believed (pp. 28-32) that phylogenetic research operated by first recognizing groups of Recent species, and secondarily placing fossils on that cladogram. We eschew the differential treatment of fossils (see also Willmann, 1987), and prefer to avoid the *groupstem group terminology. Moreover, we believe that stem groups are paraphyletic, and that $\Lambda$ x's (1985) relabeling them as stem lineages does not obviate the problem. Even Hennig recognized (1981:30) that the "stem-group concept is partly typological and not purely genealogical, and as such its use in phylogenetic research cannot be completely justified." We believe it would be instructive to re-examine Jefferies' (1986) claims concerning vertebrate ancestry, but without treating fossil cornutes and mitrates as stem groups.

It is clear that the Linnean categorical ranks attributed to the hierarchical levels in a taxonomy are arbitrary. Synapsida has been ranked as a Subclass, Therapsida an Order, Cynodontia a Suborder, and Mammalia a Class. If both Synapsida and Theria can be considered Subclasses, and Therapsida and Primates Orders, there is an obvious lack of conceptual equivalence among categorical ranks. Therefore, we have not 
employed categorical ranks in our taxonomy of $A$ mniota. The nomenclatural hierarchy we proposed is merely an idented list, a subordination of names, the less inclusive being more indented. In cladistic classification, structure of historical relationships alone designates the taxonomic hierarchy.

\section{Reasons for the Alternative Phylogenetic Hypotheses}

\section{Potential sources of new and different evidence}

Traditionally, biologists have spoken of mammals and birds as having "evolved from reptiles." Consequently, it seems that more explicitly phylogenetic questions, such as the sister group relationships of turtles, have received less attention. One might think that if neontologists had been more concerned with questions of the latter type, they might have discovered evidence for a hypothesis more closely approximating ours (Fig. 3). One must bear in mind, however, that there has been intense interest in extant amniotes since well before Darwin's time, and our review of these data (exclusive of fossils) still yielded most of Gardiner's original tree (compare Figs 1 and 2).

By the late 19th century, it was generally accepted that some "reptiles" are cladistically closer to birds and others are closer to mammals. However, little in the way of supporting evidence was published, and there existed considerable disagreement as to the relationships of what are currently termed "anapsid" amniotes, or those taxa that failed to fall neatly into one or the other group (e.g., Huxley, 1871; Baur, 1887, 1889; Cope, 1892; Woodward, 1898; Osborn, 1903; Goodrich, 1916). The hypotheses proposed by paleontologists working on both extinct and extant amniotes (Romer, 1966) bear a much greater resemblance to our hypothesis (Fig. 3) than they do to the Recent tree (Fig. 2). In fact, perhaps the most novel feature of our hypothesis is the sister group relationship between turtles and saurians. For example, Kuhn-Schnyder (1987) briefly reviewed the paleontological evidence for Gardiner's and Løvtrup's classification (Fig. 1) and, while denouncing Haemothermia as "absurd" (p. 1), he recognized mammals and saurians (his Diapsida) as sister taxa.

Why are the Recent tree and our hypothesis so different? Are they the result of using different outgroups, and the markedly different character polarities they engendered (see Doyle and Donoghue, 1987)? Extant lissamphibians and dipnoans were used as the first and second outgroups, respectively, in polarizing the Recent Data Set (Table 1). However, when considering both extant and extinct tetrapods (the Combined Data Set), the extinct diadectomorphs, seymouriamorphs, and anthracosaurs (sensu Heaton, 1980), were deemed the more appropriate outgroups, because they are cladistically closer to amniotes than are extant dipnoans and lissamphibians (Gauthier et al., 1988b).

Both Recent dipnoans (Bemis, 1984) and lissamphibians (Bolt, 1977) are believed to be paedomorphic, and such a developmental history can complicate phylogenetic analyses (Fink, 1982; Kluge and Strauss, 1985). For example, extant lissamphibians and dipnoans do not have a postorbital bone, and this state was assumed to be ancestral in the Recent Data Set. However, the bone is widespread among fossil anamniote tetrapods, and its presence was scored as ancestral in the Combined Data Set.

Our interpretation of plesiomorphy in the Recent Data Set was further complicated by employing the extant dipnoans and lissamphibians as outgroups. When a character varies in the ingroup and each of the outgroups, polarity may not be decided unambiguously according to parsimony (Farris, 1982; Maddison et al., 1984). For example, a postfrontal bone is either present or absent in both lissamphibians and 
amniotes, and its condition in dipnoans is difficult to determine (Miles, 1977: 226-245). Consequently, we had no basis upon which to choose either the presence or absence of a postfrontal as the ancestral amniote condition. Ambiguous conclusions, such as these, are usually resolved by studying additional outgroups. Numerous fossil anamniote tetrapods retain the postfrontal, and the simplest interpretation in the Combined Data Set was that its presence is plesiomorphic.

The recent and Combined Data Sets also differ in the number and kind of characters. Soft anatomical characters form the larger portion of the matrix for Recent amniotes, there being 67 soft versus 42 hard characters. In contrast, the number of hard characters increases dramatically to 207 in the Combined Data Set, while the number of soft anatomical characters remains the same.

Occasionally, additional taxa display intermediate states that lead to an increased number of transformations and a corresponding increase in synapomorphies (Doyle and Donoghue, 1987). At least superficially, this does not appear to be the major reason for the extra information in the Combined Data Set, because multistate characters account for only $7 \%(15 / 207)$ of the characters in Table 2 (including characters 96 and 97 , where the ancestor state is scored as not applicable). Intermediate additional taxa might also be important in relating comparable states that would have been overlooked because of their marked dissimilarity. Such a source of new characters might explain the much larger Combined Data Set (however, see below).

Adding relatively primitive taxa can also play a significant role in assessing the relationships of highly modified representatives of the same group. For example, $19 \%$ $(40 / 207)$ of the characters germane to amniote inter-relationships (Table 2) could not be scored in turtles owing to the profound modifications characteristic of that taxon. The placement of turtles within Amniota owes much to their sister group relationship to captorhinids (Gaffney and McKenna, 1979), and the much less modified, and thus more readily interpretable, morphology of the latter group. A more obvious example concerns mammals. Synapsid relationships were delimited by 174 skeletal synapomorphies, and most of those characters involved mammals. In an analysis that considered only relationships among extant amniotes, these synapomorphies would be overlooked or viewed as autapomorphies of mammals, and therefore ignored. Thus, we emphasize that cladistic evidence should be viewed in terms of both amount and taxonomic relevance.

\section{Further experiments}

Doubtless, each of the aforementioned factors contributed to the different results (Figs 2, 3) obtained from the Recent and Combined Data Sets. To identify their relative importance, we performed a variety of additional experiments. Our first concern was to distinguish between the importance of the ingroup (amniote) and the outgroup (anamniote choanates) fossils. Accordingly, we removed all the ingroup fossils. In such a deletion, we were applying the same data, namely the hard anatomy of Table 2 plus soft ana tomy in Table 1, to the question of Recent amniote phylogeny, but without the effect of the ingroup fossils. This analysis still yielded the Recent tree $(L=325, c=0.797)$, and demonstrates that it is the ingroup fossils, rather than the outgroup fossils and the revised polarities and new characters they indicate, that are responsible for the differences in our hypotheses (Figs 2, 3).

Two additional sets of experiments were performed on the Combined Data Set to locate the critical amniote fossils. First, we employed a coarse-grained set of taxonomic 
deletions, one in which the reptile fossils were removed from the analysis, and another in which the fossil synapsids were deleted. This procedure revealed that the evidence provided by fossil synapsids is responsible for our hypothesis (Fig. 3). Gardiner's Thecodontia resulted (Figs 1,2 ) when fossil synapsids were removed, whereas deleting fossil reptiles had no effect on our tree.

Next, we examined fossil synapsids more closely, in an attempt to identify the taxa responsible for our phylogenetic hypothesis (Fig. 3). Two series of taxonomic manipulations were performed which involved exclusion and inclusion of the fossil synapsids. Mammalia was included in all of these fine-grained experiments. First, fossil synapsids were added sequentially, from those most distantly to those more closely related to mammals, following their order in Fig. 3. Then, fossil synapsids were added in the reverse order, from those nearest to mammals to those more distant, again following the order in Fig. 3. This series of additions revealed that the evidence provided by the earliest, Casea, and latest, Morganucodontidae, fossil synapsids is insufficient to place mammals as the sister group to all other amniotes, and thus refute Gardiner's Thecodontia. Thus, we observed that a vast group of extinct synapsids, from Ophiacodon to Exaeretodon, is important in realizing our hypothesis (Fig. 3 ).

The second series of these experiments was even more detailed, because the effect of each extinct synapsid was considered one at a time. Again, we found that including Casea or Morganucodontidae was insufficient to place Mammalia as the sister group to all other Recent amniotes. Including Ophiacodon produced two equally parsimonious trees, (mammals (turtles (lepidosaurs (crocodiles, birds)))) and (turtles (lepidosaurs (mammals (birds, crocociles)))), while Tritylodontidae also resulted in two equally parsimonious cladograms, (mammals (turtles (lepidosaurs (crocodiles, birds)) ) ) and ((mammals, turtles) (lepidosaurs (birds, crocodiles $)$ )). Thus, we observed that including any one of the extinct synapsids from Edaphosaurus through Exaeretodon (Fig. 3 ) suffices to place Mammalia as the sister group to all other extant amniotes.

We also discovered that the Recent taxa did not determine the topology of our tree, because removing them yielded precisely the same branching pattern among the fossils as those illustrated in Fig. 3. Moreover, Gardiner's Thecodontia still emerged from the analysis when only latest Triassic through Recent amniotes were included.

Given our hypothesis (Fig. 3), it is evident that the characters supporting the sister group status of $\Lambda$ rchosauromorpha and Mammaliformes are most parsimoniously interpreted as convergences. A closer examination of the evidence supporting this grouping reveals that $80 \%$ of the unambiguously optimized characters pertain to the locomotor system, in spite of the fact that postcranial characters account for only $40 \%$ of the evidence in Table 2. Furthermore, half of the unambiguous soft anatomical characters supporting this group are also related to locomotion (e.g., the initial elaboration of the cerebellum). Locomotor specializations have long been used to characterize the evolutionary history of Amniota (Romer, 1956; Kemp, 1982), and the relative importance of locomotion and nonlocomotion characters in our study is consistent with that bias.

\section{The Importange of Additional Taxa-Generalizations}

Exactly what evidence do the critical, fossil synapsids provide that enabled them to play such a pivotal role? We employ a simple hypothetical example to demonstrate, in general, what minimal characteristics an additional taxon or taxa must have to overturn 

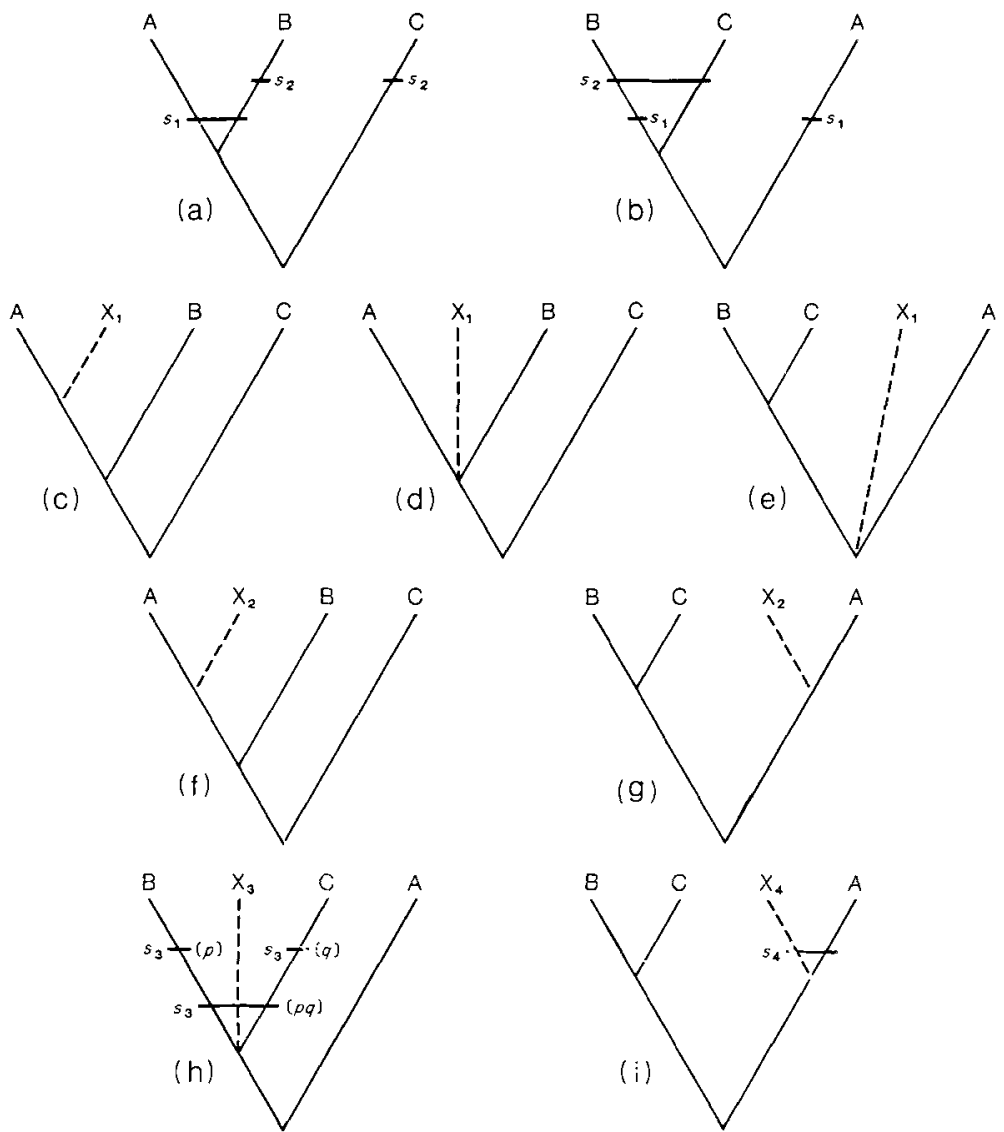

Fig. 4. The most parsimonious cladograms that can be obtained from Table 3 (assuming the optimization protocol of Farris, 1970). Without additional taxa $\left(\mathrm{X}_{z_{-4}}\right)$, cladograms (a) and (b) are equally parsimonious. Equally parsimonious outcomes ( $(c-c)$ and $(f-g)$, respectively) remain when $X_{1}$ or $X_{2}$ are added. Only $X_{3}$ and $\mathrm{X}_{4}$ provide unique, most parsimonious solutions $(\mathrm{h})$ and (i), respectively). See text for further explanation.

a phylogenetic hypothesis. Consider equally most parsimonious cladograms (Fig. $4 a-b$ ) derived from a hypothetical data matrix (Table 3 ), which consists of three terminal taxa $(A, B$, and $C)$ and their most recent common ancestor $(\Lambda N C)$. If one thinks of taxon $A$ as mammals, taxon $B$ as archosaurs, and taxon $C$ as either lepidosaurs or turtles, the hypothetical example is like the amniote case history in question; the $(\mathrm{A}, \mathrm{B})$ and $(\mathrm{B}, \mathrm{C})$ alternative groupings are similar to Figs 2 and 3, Gardiner's Thecodontia and our Reptilia, respectively. Only four characters, $s_{1-4}$, are listed in the matrix; most autapomorphies have been eliminated for simplicity. The " 0 " states are ancestral, the other conditions derived therefrom. Only when derived states are shared is there evidence for two taxa being each others' closest relatives. Four taxa, $\mathrm{X}_{1-4}$, each exhibiting a different pattern of primitive and derived states, are considered "additions" to the matrix (Table 3), and in that regard they are equivalent to the fossils we employed in our study of amniote relationships. A new best-fitting cladogram is sought with each of these additions. When a unique parsimony solution is found, viz. either $(A, B)$ or $(B, C)$ is delimited, the taxon added during that run is deemed important in the context of this example; otherwise it is considered unimportant. 
Table 3

Hypothetical data set.

\begin{tabular}{cccccc}
\hline & & \multicolumn{4}{c}{ Characters } \\
\cline { 3 - 6 } & & $s_{1}$ & $s_{2}$ & $s_{3}$ & $s_{4}$ \\
\hline & Anc & 0 & 0 & 0 & 0 \\
$\mathrm{~T}$ & $\mathrm{~A}$ & 1 & 0 & 0 & 1 \\
$\mathrm{a}$ & $\mathrm{B}$ & 1 & 1 & $p$ & 0 \\
$\mathrm{X}$ & $\mathrm{C}$ & 0 & 1 & $q$ & 0 \\
$\mathrm{a}$ & $\mathrm{X}_{1}$ & 1 & 0 & $\mathrm{~N}$ & 0 \\
& $\mathrm{X}_{2}$ & 1 & 0 & $\mathrm{~N}$ & 1 \\
& $\mathrm{X}_{3}$ & 0 & 0 & $p q$ & 0 \\
& $\mathrm{X}_{4}$ & 0 & 0 & $\mathrm{~N}$ & 1 \\
\hline
\end{tabular}

The transformation series in $s_{q}$, considering only Recent taxa $(\mathrm{A}, \mathrm{B}, \mathrm{C})$, is $p<-0->q$. Adding the taxon $\mathrm{X}_{3}$, the hypothesis of character evolution becomes $0 \cdots p q>p$ (and $p q \cdots>q$, independent of $p)$.

Implementing this model illustrates that there is no importance attached to the additional taxon, $\left(\mathrm{X}_{1}\right)$, that exhibits the synapomorphy $\left(s_{1}\right)$ of one of the two competing clades, $(\mathrm{A}, \mathrm{B})$ or $(\mathrm{B}, \mathrm{C})$; equally parsimonious hypotheses remain (Fig. 4c-e). Further, an additional taxon, $\left(\mathrm{X}_{2}\right)$, is unimportant when it is relatively derived $\left(s_{1}\right.$ and $\left.s_{4}\right)$ and found to be the sister group of any of the terminal taxa, A or B or C (Fig. 4f-g). Taxa with these properties might be termed apomorphic sister groups.

An additional taxon, $\left(\mathrm{X}_{3}\right)$, is important when it leads to the discovery of a synapomorphy corroborating $(A, B)$ or $(B, C)$. Taxa of his sort, that will hereafter be referred to as plesiomorphic sister groups, serve as sources of new evidence, because of their intermediacy. For example, states $p$ and $q$ of $s_{3}$ are interpreted as autapomorphies among the members of the ingroup until the intermediate state $p q$ is observed in $\mathbf{X}_{3}$ (Table 3). Taxon $\mathrm{X}_{3}$ can be thought of as establishing an empirical basis for inferring an intermediate level of generality (pq) between 0 and $p$ and $q$ (Fig. 4h). Intermediate taxa may relate states previously hypothesized to have had an independent and earlier origin in the outgroup. Intermediate taxa may even call attention to comparable states that were overlooked altogether because of their great dissimilarity. It is in these ways that $\mathrm{X}_{3}$ is important, and the more intermediate characters a taxon exhibits, the more effective it will be in this respect (Doyle and Donoghue, 1987).

One might be tempted to attribute special significance to fossils because the vast majority of all species are extinct, and therefore, all other things being equal, fossils form a potentially larger resource from which to sample intermediates. Attributing special significance to fossils because of their intermediacy entails at least two unrealistic assumptions. One must assume that all organisms are equally fossilizable, and that extinct and extant species are equally informative regarding characters. Obviously, organisms vary both in the fossilizability of their parts, and in the degree to which the environments of their habitation may be represented in the fossil record. Even under the best of conditions, fossils are incomplete samples of the phenotype-usually markedly so relative to representatives of extant species. The incomplete nature of fossils means that fewer potential synapomorphies are observable, even in those organisms that may be fossilized, and their potential for intermediacy is thereby considerably diminished. 
Furthermore, clues to intermediacy might just as well come from embryology, without having to survey any additional taxa, extinct or extant. While some transformations can actually be observed in ontogeny, one or more assumptions are required to be able to relate those changes to descent with modification (Kluge, 1985).

Our simple model also reveals that relatively primitive forms, plesiomorphic sister taxa, are important. For example, $\mathrm{X}_{4}$ shares an apomorphy $\left(s_{4}\right)$ with one of the members $(\Lambda)$ of a derived clade (A, B), but it is primitive otherwise $\left(s_{1,2}\right)$. Adding $X_{4}$ "forces the removal" of $A$ from its sister group relationship with $B$, thereby leaving $(\mathrm{B}, \mathrm{C})$. Retaining the (A, B) hypothesis would be less parsimonious, because one of the primitive states $\left(s_{1}\right)$ of $X_{4}$ would have to be interpreted as an evolutionary reversal.

Which of our generalizations, Fig. 4 h or 4i, explains the importance of the pivotal, fossil synapsids in our study of amniote phylogeny? The answer is clear. Adding those fossils does not increase the evidence for Reptilia $(B, C)$, exclusive of Mammalia $(\Lambda)$, as per Fig. 4h. Whatever intermediacy the fossils might exhibit does not translate into additional evidence for Reptilia, because they cluster well within the collateral taxon Synapsida (Fig. 3). Parenthetically, we note that the additional fossil reptile taxa|are not significantly intermediate because they had no effect on determining the placement of Mammalia (Gauthier et al., 1988b). To test the other possibility (Fig. 4i), we calculated the number of reversals (analogous to $s_{1}$ in the hypothetical example, Table 3, Fig. 4) exhibited by the pivotal, fossil synapsids $\left(=X_{4}\right)$ when they are placed as a sister group to Archosauromorpha (B). The number of evolutionary reversals should be greater in that context $\left(\mathrm{B}, \mathrm{X}_{4}\right)$ than when the critical synapsids (Edaphosaurus through Exaerelodon; Fig. 3$)$ are related to mammals $\left(\Lambda, \mathrm{X}_{4}\right)$. Indeed, reversals increased by a factor of 2.4 ( 7 to 17 ) to 3.8 (14 to 53), excluding those reversals confined to the terminal taxa. The range of values resulted from using different optimization schemes in PAUP, DELTRAN and FARR IS respectively (Swofford, 1984). The pivotal synapsids share 57 unique and unreversed apomorphies with Mammalia $\left(=s_{4}\right)$, unordered characters omitted. Thus, we conclude that the importance of the additional synapsids in question is due to their sharing many apomorphies with mammals (57), but being significantly primitive otherwise (in $10[17-7]$ to $39[53-14]$ features).

\section{The Parallelism Between Phylogeny and the Fossil Record}

$\Lambda \mathrm{n}$ ancestor arises before its descendants, although they may overlap one another in time (Darwin, 1859; Hennig, 1966, 1981). This condition seems to underlie our expectation of a positive correlation between the temporal order implied by the fossil record and a phylogenetic hypothesis. However, there are diverse and reasonably well understood reasons why such correlations might be less than perfect (e.g. Van Andel, 1981; Novacek and Norell, 1982; Dingus and Sadler, 1982; Behrensmeyer and Schindel, 1983). An incorrect hypothesis of relationship can account for a poor correspondence with stratigraphic data, but more often than not such discordance is attributed to the plethora of factors contributing to incomplete sampling of fossil taxon-ranges. Particularly important processes affecting fossils are erosion, metamorphosis, and diagenesis. Ancient groups present considerable difficulties, because more and larger gaps are to be expected in the fossil record with increasing age (Sadler, 1981; Sadler and Dingus, 1982; Dingus and Sadler, 1982); the further back in time a species really was extant, the greater are the chances that no trace of it will remain.

Nevertheless, the order implied by the stratigraphic occurrence of amniote fossils, together with the cladistic rank inferred from our hypothesis (Fig. 3), enabled us to 
describe the association between phylogeny and the fossil record (Hill and Camus, 1986a). Each of the 29 amniote terminal taxa was assigned an age based on its first appearance in the fossil record, and rank-ordered according to that criterion. Cladistic rank was defined as the number of cladogenic events (nodes) a terminal taxon is removed from the most recent common ancestor of all extant amniotes. These data were then arcsine transformed. Given all the reasons one might expect incongruence, it is striking that comparing our tree with the fossil record yielded a correlation coefficient $(r)$ of 0.679 , which is significant at $P>0.01(\mathrm{df}=27)$. Obviously, the synapsid data are much more strongly correlated than the reptile data (Fig. 5a). Early synapsids show some anomalies, but not to the degree seen in early reptiles, in general, and some early archosauromorphs, in particular. The placement on the tree of Rhynchosauria, Trilophosaurus and Choristodera suggests much earlier ages of origin than the fossil record currently indicates and the highly specialized morphology of the known representatives of these taxa is consistent with that thesis.

Following this method of comparing cladistic rank and age of origin, which phylogeny, ours (Fig. 3) or Gardiner's original formulation (1982, his figs 3 and 4), provides a better fit to the fossil record? This comparison is complicated by our having considered different terminal taxa. In order to place the competing hypotheses on as even a footing as possible, we reduce the cladograms to just those 17 terminal taxa common to both (see legend to Fig. 5). Fig. 5b and $\mathrm{c}$ indicate that our phylogenetic hypothesis is more strongly correlated with the fossil record $(r=0.868$ and $r=0.584$, respectively; the former is significant at $P>0.01$, the latter at $P>0.05)$. Comparing competing classifications in some statistical manner such as this seems to avoid Platnick's (1986a: 292) major criticisms of Hill and Camus' (1986a) use of stratigraphy to test phylogenetic hypotheses (see also Hill and Camus, 1986b; Platnick, 1986b).

Size bias may be responsible for the poor correlation between stratigraphic and cladistic rank observed among the earliest amniotes. Reisz (1972) noted that size increase was one of the most distinctive trends in early synapsid evolution; the contemporaneous reptiles remained small. Since larger fossils are generally easier to discover than are smaller ones, this difference could explain the closer correlation observed among the Paleozoic synapsides (Fig. 5a). Other less obvious biases in the fossil record also may affect our understanding of the times of divergence of early amniotes.

Continental deposits predominate in the later part of the Pennsylvanian, but they record mainly the wet-adapted fauna of the coal swamps (Romer, 1966). Late Pennsylvanian (Stephanian) amniotes, such as sphenacodontines and araeoscelidians, demonstrate that the lineages surviving as mammals, turtles and saurians had already diverged from one another by that time. Earlier Pennsylvanian deposits are largely continental, but drier habitats from which we might expect to sample the earliest amniotes are rare. In fact, our record of early amniotes is confined almost entirely to the contents of upright lycopod stumps at Florence and Joggins, Nova Scotia (Romer, 1966). The stumps at Florence contain remains of the synapsid Archaeothyris (Reisz, 1972) and the reptile Paleothyris (Carroll, 1969), thus pointing to a reptile-synapsid split by at least the mid-Pennsylvanian (Westphalian D). Westphalian B fossil tetrapods from lycopod stumps at Joggins, including the fragmentary remains of possible reptiles (Hylonomus; Carroll, 1964) and synapsids (Protoclepsydrops; Carroll, 1964), suggest a still older divergence in the early Pennsylvanian. Earlier Carboniferous deposits are dominated by marine sediments (Romer, 1966), thus making discovery of examples of the most ancient amniotes unlikely. 


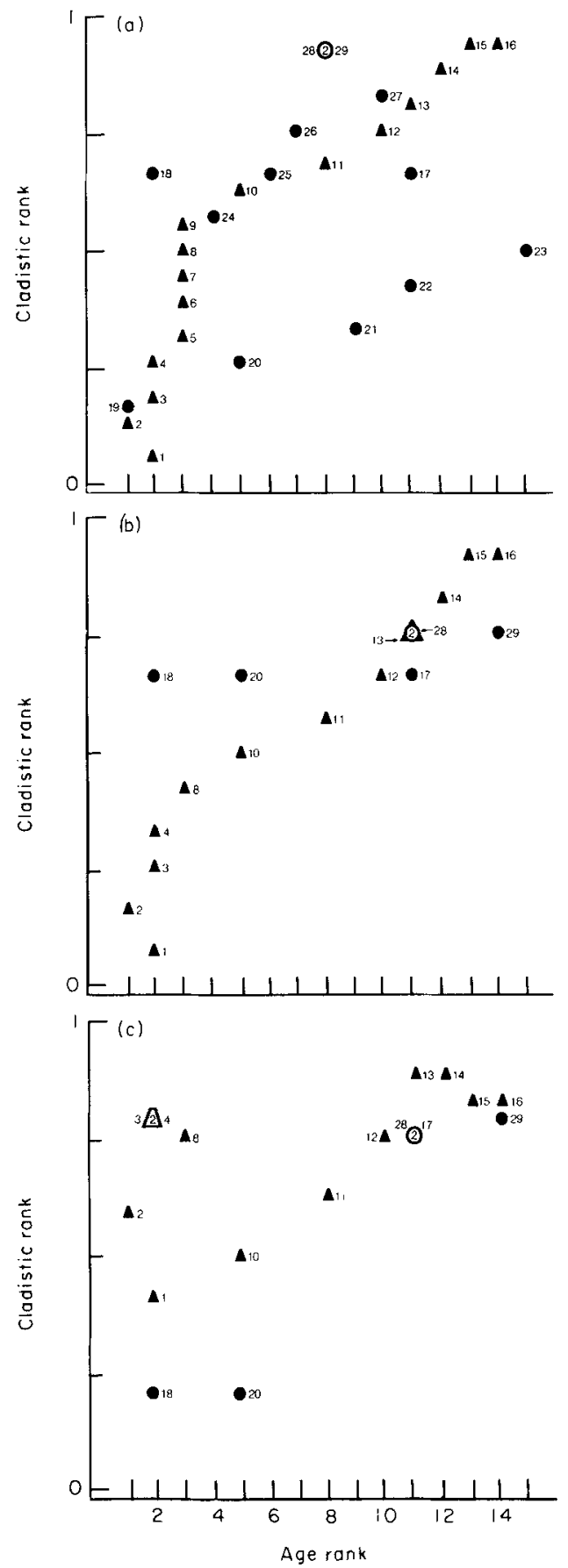




\section{Missing Data: Are Fossils Always Less Informative than Living Taxa?}

Obviously, fossils cannot offer as many potential sources of evidence as can extant taxa because they represent only parts of organisms (Hennig, 1966). But to say that extant taxa could often be more informative is not to conclude that they always will be. Indeed, our study reveals that extant taxa may be even less informative, or for that matter unimportant in determining amniote phylogeny, in cases where the living representatives are widely divergent from one another. Perhaps the shortcomings of fossils have been emphasized while the effects of evolution and extinction on the information available in extant forms have been overlooked.

This point is illustrated by comparing Recent mammals with the late Carboniferous sister group of all other synapsids considered in this analysis, the Varanops-Casea, or caseoid, clade. As one would expect, we know nothing of the 67 soft anatomy characters in caseoids. Fossils are usually incomplete, and caseoids are also missing information for four skeletal characters. Thus, $26 \%$ of the relevant characters are missing in caseoids (71/274); this is the sense in which we think of fossils as being deficient in character information. Missing evidence due to nonpreservation is represented by a question mark, such as in the case of the presence of a postorbital-squamosal contact in caseoids (see Table 2, character 13).

Mammals are not missing data in the same manner in which caseoids are because all systems are available for study. Nevertheless, evolution itself has effectively produced missing data in mammals. In mammals, missing data are more apt to reside in those characters that are present, but so transformed that their relations to conditions in other amniotes cannot be ascertained. For example, none of the several characters involving the quadrate (59-63 in Table 2) can be observed in mammals, because the ancestral suspensorium has transformed into a middle ear ossicle. Consequently, the states of these characters in mammals are scored as an $\mathrm{N}$, meaning that the character does not apply. Although stemming from very different sources, both $\mathrm{N}$ and ? must be considered operationally equivalent, namely, as missing data.

Considering only the skeleton in this context, mammals are effectively missing data in $20 \%(41 / 207)$ of the characters. (An additional 27 characters are N's in all but a few living mammals; prehistoric extinction of a few key species would have resulted in as much as one-third of the 274 Combined Data Set characters being scored as N's in mammals.) It is remarkable that we could be missing data for at least $20 \%$ of the mammalian skeleton, even when the entire system, from embryo to adult, is available for study. Samples of ontogenetic stages of caseoids are more restricted, with individual

Fig. 5. The relationship between age and cladistic ranks. Age is first appearance in the fossil record, and cladistic rank is the number of nodes a terminal taxon is removed from the most recent common ancestor of all extant amniotes. Cladistic rank has been rescaled to a range of $0-1$. Triangles are synapsids, circles are reptiles. The number of co-occurring terminal taxa is indicated within a symbol. The numbers located outside the symbols denote the following terminal taxa: $\mathrm{I}=$ caseids + varanopsids; $2=$ Ophiacodon; $3=$ Edaphosaurus; $4=$ Sphenacodontinae; $5=$ Biarmosuchia; $6=$ Dinocephalia; $7=$ Gorgonopsia; $8=$ Dicynodontia; $9=$ Therocephalia $; 10=$ Procynosuchus $; 11=$ Thrinaxodon $; 12=$ Diademodon $; 13=$ Exaeretodon $; 14=$ Tritylodontidae; $15=$ Morganucodontidae; $16=$ Mammalia; $17=$ Testudines; $18=$ Captorhinidae; $19=$ Araeoscelidia; $20=$ Lepidosauromorpha; $21=$ Rhynchosauria; $22=$ Trilophosaurus; $23=$ Choristodera; $24=$ Protorosauria; $25=$ Proterosuchidae; $26=$ Erythrosuchidae; $27=$ Proterochampsidae; $28=$ Pseudosuchia; $29=$ Ornithosuchia Panel (a) is based on the cladistic hypothesis illustrated in Fig. 3. Panel (b) contains only those terminal taxa whose composition is the same as taxa recognised by Gardiner (1982; his figs 3 and 4). Cladistic rank in panel (b) was derived from our cladogram (Fig. 3), whereas cladistic rank in panel (c) was extracted from Gardiner's hypothesis (his figs 3 and 4). See text for further explanation. 
specimens often incomplete and poorly preserved. Nevertheless, barely $2 \%$ (4/207) of the amniote skeletal characters remain unknown or uninterpretable in caseoids. Comparing the total number of missing characters in the earliest and latest synapsids in the Combined Data Set of 274 characters, caseoids are missing 26\% (71) and mammals are missing $15 \%(41)$. The difference between $26 \%$ and $15 \%$ supports the contention that extant taxa offer more characters than do extinct taxa at this level of analysis. However, the mere $11 \%$ increase provided by mammals indicates that having survived to the Recent does not guarantee that a taxon will provide a substantially greater amount of relevant morphological information.

Our examination of amniote phylogeny indicates that although there might be additional sources of evidence in the Recent biota, that circumstance does not confer upon these data any special ability to resolve phylogenetic hypotheses at every level of analysis. We noted above that some skeletal synapomorphies listed in the Recent matrix (Table 1) are patently absurd, in terms of taxonomic generality and/or polarity, when considered in light of extinct taxa. However, the inadequacy of these hard characters becomes apparent only in the light of the denser sample of morphology afforded by extinct tetrapods; comparable knowledge of soft anatomy in fossils might make some putative synapomorphies in this class of characters seem equally absurd.

Some appreciation for the possible effects of a denser sample can be obtained from comparing the hard characters in Tables 1 and 2. For purposes of discussion, we assume that those hard characters that are congruent with our tree (Fig. 3) are "true" and those showing discordance are "false." Only four of the 39 unambiguously optimized hard characters in Table 1 are "true" by this criterion; among extant amniotes, archosaurs do indeed possess a laterosphenoid ossification, foramen aerosum (siphonial system), mandibular fenestra, and vertebral spine tables. Another ten skeletal characters in Table 1 also appear to be unique and unreversed, if one looks only at the Recent taxa, but they are not exactly "truc." Additional evidence from fossils indicates that polarity should be reversed in two characters (G11a, A46). Further, the remaining eight are convergent characters arising within pseudosuchians and ornithosuchians; they only appear to be synapomorphies uniting extant crocodilians and birds (G19a, G26, A22, A23, A27, A44, A48, A53). These caveats aside, a total of $36 \%(14 / 39)$ of the unambiguously optimized hard characters in Table 1 appear, in the context of Recent taxa only, to be "true;" $64 \%$ appear "false."

Soft characters are generally not scoreable on extinct taxa, so there is no simple way to compare their verity to the hard characters. However, assuming that hard characters are no more or less liable to homoplasy than are soft characters, as many as $64 \%$ of the soft anatomy characters could also be "false." This proposition can be tested by comparing the expected number of "true" versus "false" soft characters derived from the preceding analysis, to the number of "true" versus "false" soft characters observed among the 62 unambiguously optimized soft characters distributed on our tree. Thus, we expect to see $22(0.36 \times 62)$ "true" and $40(0.64 \times 62)$ "false" soft characters. We observe that 26 are perfectly consistent characters ("true") and that 36 are inconsistent ("false"). There is no significant difference between the expected and observed numbers (Chi-square $=0.544$ ). Fossilizable characters per se do not appear to afford any clearer picture of amniote phylogeny than do those that are not likely to be found in fossils. Nevertheless, hard characters can be assessed in the context of a far denser sample of relevant amniotes than can soft characters; one must not accept uncritically the reliability of characters, such as physiological features or DNA sequences, that are 
determinable only in that fraction of amniote diversity that happens to have survived to the Recent.

\section{The Importance of Fossils}

Lastly, why should fossils be more primitive than extant taxa, as in Fig. 4i? The simplest explanation for the more primitive nature of fossils is that they had less time to evolve. This conservative aspect of fossils has been alluded to by others. For example, Patterson and Rosen (1977: 155) observed that "the older the fossil the greater chance it will represent a plesiomorphic sister-group."

As mentioned above, evolutionary theory requires that the earliest species of a taxon cannot be more derived than its descendants. Nlthough there is no reason to expect a one-to-one correspondence, it is widely recognized that there should be some positive correlation between the amount of time and the degree of evolutionary change (e.g., Britten, 1986). Thus, if we assume that extinct species, relative to extant sister species, have had less time to evolve, then as a rule they will be more primitive (Darwin, 1859). We might also expect, for groups that have undergone considerable modification and extinction, that "the older members should differ less from each other in some of their characters than do the existing members of the same groups" (Darwin, 1859:360-361). This is certainly the case for amniotes; extant synapsids differ by at least 156 synapomorphies from extant reptiles, but the early members of these clades differ by no more than ten. Of course, there is no reason to expect that the dichotomy between more and less primitive will always correspond to that between extinct and extant taxa (Darwin, 1859). The expectation of greater primitiveness is warranted only in the case of sister taxa. Furthermore, significant differences are expected to obtain only in groups that are characterized by at least moderate amounts of evolution and extinction. Thus, fossils should be most important in phylogenctic inference when the group of interest is old and only a few, highly modified, terminal taxa are extant. This is exactly the situation in amniotes: mammals have not shared a common ancestor with the rest of the amniotes since at least the middle of the Carboniferous; more than $80 \%$ of the amniote terminal taxa are extinct; and the extant terminal taxa are profoundly different from one another.

Some biologists have belittled the role of fossils in phylogenetic inference, because they are only parts of organisms. Missing data can be a problem, but it is not one to which extant taxa are immune. Clearly, extant taxa can offer a greater number and variety of characters than can extinct taxa. But this does not justify equating the mere amount of evidence with its relevance. $\Lambda$ character can be germane to phylogenetic questions at one level of analysis and not so at another. It makes no sense to consider fossil (or living) organisms to be either more or less informative without also specifying the level of generality of the question posed. We contend that in a particular case, the phylogenetic position of mammals among amniotes, evidence from fossils is of greater relevance than the phenotypic diversity available only from Recent taxa. Even without such an example, we would advise systematists to avoid prejudging the importance of one kind of organism over another, in effect thinking typologically, and consider all of the evidence regardless of its source.

\section{Acknowledgments}

The coauthors wish to acknowledge the assistance of James M. Clark, William R. Dawson, Llewellyn D. Densmore, Herbert C. Dessauer, Byron A. Doneen, Eugene S. 
Gaffney, Chris Gow, James Hopson, Ronald Lawson, John Maisey, Michael M. Miyamoto, Ronald A. Nussbaum, Anita K. Pearson, Robert Presley, Robert Storer, and Marvalee $H$. Wake. Our overview of amniote synapomorphies could not have been completed without their special knowledge. R. Glenn Northcutt was particularly generous with his knowledge of anatomy, and we are very grateful to him. We also wish to recognize the assistance of the members of the University of Michigan Systematics Discussion Group where this paper was first presented. Their critical comments helped to shape the basic approach we have taken. Kevin de Queiroz, Steve Farris, Thorkil Feilberg, Sara Fink, Jim Hopson, Eric Lombard, Mary McKitrick, Colin Patterson, Paul Sereno, Jim Sprinkle, Hans Sues, Marvalee Wake, John Wible, and Bob Zanon read all, or major parts, of the manuscript. Their editorial comments and questions concerning philosophy, methodology and anatomy greatly improved the final document. We assume full responsibility for all errors of commission and omission that remain. The study was supported by National Science Foundation grants BSR-83-04581 (to A.G.K.) and BSR-84-13847 (to T.R.).

\section{REFERENGES}

Ax, P. 1985. Stem species and the stem lineage concept. Cladistics 1: 279-287.

BAdenhorst, A. 1978. The development and the phylogeny of the organ of Jacobsen and the tentacular apparatus of Ichthyophis glutinosus (Linne). Ann. Univ. Stellenbosch, Ser. 2, 1: $1-26$.

Badham, J. A. 1971. Albumen formation in eggs of the agamid Amphibolurus barbatus barbatus. Copeia (3): 543-545.

BaIRd, I. L. 1970. The anatomy of the reptilian ear. In Gans, C., and T. S. Parsons (eds.), Biology of the Reptilia. Vol. 2 (Morphology B). Acad. Press, New York, pp. 193-275.

Baldwin, J., and P. 'Temple-Smith. 1973. Distribution of LDHX in mammals: Presence in marsupials and absence in the monotremes platypus and cchidna. Comp. Biochem. Physiol. 44B: 805-811.

Ballard, W. W. 1964. Comparative anatomy and embryology. Ronald Press, New York.

Barone, R. 1972. Appareil circulatoric. In Grasse, P.-P. (ed.), 'Traite de zoologie. Vol. 16, Masson, Paris, pp. 704-921.

Baumel, J. J. 1979. Systema cardiovasculare. In Baumel, J. J., A. S. King, A. M. Lucas, J. E. Breazile, and H. E. Evans (eds.), Nomina anatomica avium. Academic Press, London, pp. 343-407.

Baumel, J. J., and L. Gerchman. 1968. The avian intercarotid anastomosis and its homologue in other vertebrates. Amer. J. Anat. 122: 1-18.

Baur, G. 1887. On the phylogenetic arrangement of the Sauropsida. J. Morph. 1: 93-104.

BaUR, G. 1889. On the morphology of the vertebrate skull. J. Morph. 3: 471-474.

BEDvard, F. E. 1905. Some contributions to the knowledge of the anatomy, principally of the vascular system, of Hatteria, Crocodilus and certain lizards. Proc. Zool. Soc. London I905: $461-489$.

Beddard, F. E., and P. C. Mitchell. 1895. On the structure of the heart of the alligator. Proc. Zool. Soc. London 1895: 343-349.

Behrensmeyer, A. K., and D. Schindel. 1983. Resolving time in paleobiology. Paleobiology 9: $1-8$.

Bellairs, A. D'A. 1970. The life of reptiles. Universe Books, New York.

Bellairs, A. D'A., ANd C. R. Jenkin. I960. 'The skeleton of birds. In Marshall, A. J., (ed.), Biology and comparative physiology of birds. Vol. 1. Academic Press, New York, pp. 241-300.

Bellairs, A. D'A., AND A. M. Kamal. 1981. The chondrocranium and the development of the skull in Recent reptiles. In Gans, C. and T. S. Parsons (eds.), Biology of the Reptilia. Vol. 11 (Morphology F). Academic Press, New York, pp. 1-263.

Bemis, W. E. 1984. Paedomorphosis and the evolution of the Dipnoi. Paleobiology 10: 293-307. 
Benninghoff, A. 1933. Herz. In Bolk, L., et al., (eds.), Handbuch der vergleichenden Anatomie der Wirbeltiere. Vol. 6. Urban and Schwarzenberg, Berlin, pp. 467-556.

Bentley, P. J. 1976. Comparative vertebrate endocrinology. Cambridge Univ. Press, Cambridge.

Benton, M. J. 1983. The Triassic reptile Hyperodapedon from Elgin: functional morphology and rclationships. Phil. Trans. Roy. Soc. London 302(B): 605-717.

Benton, M. J. 1984. The relationships and early evolution of Diapsida. In Ferguson, M. W. J. (ed.), The structure, development, and evolution of reptiles. Academic Press, London, pp. 575-596.

Benron, M. J. 1985. Classification and phylogeny of the diapsid reptiles. Zool.J. Linnean Soc. 84: 97-164.

BerG, L. S. 1926. Nomogenesis or evolution determined by law. Constable, London. (Translated from Russian by J. N. Rostovtsov.)

Berger, P. J., and G. Burnstock. 1979. Autonomic nervous system. In Gans, C., G. Northcutt, and P. Ulinski (cds.). Biology of the Reptilia. Vol. 10 (Neurology B). Academic Press, London, pp. 1-57.

Bertmar, G. 1965. 'The olfactory organ and upper lips in Dipnoi, an embryological study. Acta Zool. Stockholm 46: 1-10.

BerTmar, G. 1966. The development of the skeleton, blood-vessels and nerves in the dipnoan snout, with a discussion on the homology of the dipnoan posterior nostrils. Acta Zool. Stockholm 47: 81-150.

Beuchat, C. A. 1986. Phylogenetic distribution of the urinary bladder in lizards. Copeia (2): $512-517$.

Bhatia, M. I. 1929. On the arterial system of the lizard Uromastix hardwickii Gray. J. Morph. Physiol. 48: 281-292.

Bockman, D. E. 1970. The thymus. In Gans, C., and T. S. Parsons (eds.), Biology of the Reptilia. Vol. 3 (Morphology C). Academic Press, London, pp. 111-133.

BoLt, J. R. 1977. Dissorophoid relationships and ontogeny, and the origin of the Lissamphibia. J. Paleo. 51: 235-249.

Born, G. 1876. Uber die Nasenholen und der Thranennasengang der Amphibien. Morph. Jahrb. 2: $577-646$.

Braun, E. J., and W. H. Dantzler. 1972. Functions of mammalian-type and reptilian-like nephrons in kidney of descrt quail. Amer. J. Physiol. 222: 617-629.

BRITTEN, R.J. 1986. Rates of DNA sequence evolution differ between taxonomic groups. Science 231: 1393-1398.

Broman, I. 1921. Über die Entwicklung der konstaten grosseren Nasenhohlendrusen der Nagetiere. Zeit. Anat. Entwickl. 60: 439-586.

Broom, R. 1921. On the structure of the reptilian tarsus. Proc. Zool. Soc. London 1921: 143-155.

Brown, G. W., And P. P. Cohen. 1969. Activities of urea-cycle enzymes in various higher and lower vertebrates. Biochem. J. 75: 82-91.

Butler, G. W. 1889. On the subdivision of the body-cavity in lizards, crocodiles, and birds. Proc. Zool. Soc. London 1889: 452-474.

Butler, G. W. 1892. On the subdivision of the body-cavity in snakes. Proc. Zool. Soc. London 1892: 477-498.

Cajal, S. R. y. 1911. Histologie du Systeme Nerveux de l'Homme et des Vertebres (1. Azoulay, trans.). Vol. 2. Maloine, Paris.

Camp, C. L. 1930. A study of the phytosaurs. Mem. Univ. California 10: 1-61.

Carrick, F. N., and R. L. Hughes. 1978. Reproduction in male monotremes. Aust. Zool. 20: 211-231.

Garroll, R. L. 1964. The earliest reptiles. J. Linnean Soc. London (Zool.) 45: 61-83.

Garroll, R. L. 1969. A middle Pennsylvanian captorhinomorph and the interrelationships of primitive reptiles. J. Paleo. 43: 151-170.

CARTER, G. S. 1967. Structure and habit in vertebrate evolution. Univ. Washington Press, Seattle.

Clark, J. M., AND P. S. Ulinski. 1984. A Golgi study of anterior dorsal ventricular ridge in the alligator, Alligator mississippiensis. J. Morph. 179: 153-174. 
Clarke, W. C., and H. A. Bern. 1980. Comparative endocrinology of prolactin. In Lee, G. H. (ed.), Hormonal protcins and peptides. Vol. 5. Acadcmic Press, New York, pp. 106-197.

Cooper, E. L. 1973. The thymus and lymphomyeloid system in poikilothermic vertebrates. In Davies, A. J. S., and R. L. Carter (eds.), Contemporary topics in immunobiology. Vol. 2. Plenum Press, New York, pp. 13-38.

Cooper, E. L. 1976. Comparative immunology. Prentice-Hall, Englewood Cliffs, New Jersey.

Cooper, E. L., A. E. Klempau, and A. G. Zapata. 1985. Reptilian immunity. In Gans, C., F. Billett, and P. F. A. Maderson (eds.), Biology of the Reptilia. Vol. 14 (Development A). John Wiley, New York, pp. 599-678.

COPE, E. D. 1869. Synopsis of the extinct Batrachia, Reptilia and Aves of North America. Trans. Amcr. Philos. Soc. 14: 1-252. (Part published in 1869, remainder in 1871.)

Cope, E. D. 1892. On the homologies of the posterior cranial arches in the Reptilia. 'Trans. Amer. Phil. Soc. 17: 11-26.

Crosby, E. C., AND 'T. Humphrey. 1939a. A comparison of the olfactory and accessory olfactory bulbs in certain representative vertebrates. Pap. Mich. Acad. Sci. 24: 95-104.

Crosby, E. C., and 'T. Humphrey. 1939b. Studies of the vertebrate telencephalon. I. The nuclear configuration of the olfactory and accessory olfactory formations and of the nucleus olfactorius of certain reptiles, birds, and mammals. J. Comp. Neurol. 71: 121-213.

Crowson, R. A. 1970. Classification and biology. Aldine Publ. Co., Chicago.

Crush, P. J. 1984. A late Upper Triassic sphenosuchid crocodilian from Wales. Paleontology 4: $131-157$.

Currie, P.J. 1985. Cranial anatomy of Stenonychosaurus inequalis (Saurischia, Theropoda) and its bearing on the origin of birds. Canad. $J$. Earth Sci. 22: 1643-1658.

Darwin, C. R. 1859. The origin of species by means of natural selection. A. L. Burt, New York. (Reprinted from the sixth London edition, 1872, with all additions and corrections.)

DawBin, W. H. 1982. The tuatara Sphenodon punctatus: aspects of life history, growth and longcvity. Occ. Publ. New Zealand Wildlife Serv. (2): 237-250.

DE BEER, G. R. 1937. The development of the vertebrate skull. Clarendon Press, Oxford.

DE BEeR, G. R. 1949. Caruncles and egg-tceth: some aspects of the concept of homology. Proc. Linnean Soc. London 161: 218-224.

de Beer, G. R. 1958. Embryos and ancestors. Clarendon Press, Oxford.

DE Querroz, K. 1985. The ontogenctic method for determining character polarity and its relevance to phylogenetic systematics. Syst. Zool. 34: 280-299.

Dendy, A. 1899. Outlines of the development of the tuatara, Sphenodon (Hatteria) punctatus. Quart. J. Microscopical Sci. 42: 1-87.

Dessauer, H. C. 1970. Blood chemistry of reptiles: physiological and evolutionary aspects. In Gans, C., and T. S. Parsons (eds.), Biology of the Reptilia. Vol. 3 (Morphology C). Academic Press, London, pp. 1-72.

Dincus, L., and P. M. Sadrer. 1982. The effects of stratigraphic completeness on estimates of evolutionary rates. Syst. Zool. 31: 400-412.

Doyle, J. A., And M. J. Donoghue. 1987. The importance of fossils in clucidating sced plant phylogeny and macroevolution. Rev. Paleobotany \& Palynology 50: 63-95.

Duelluman, W. E., and L. T'rueb. 1986. Biology of amphibians. McGraw-Hill, New York.

Duerden, J. E. 1922. The origin of feathers from the scales of reptiles. S. Afr. J. Sci. I9: 263-268.

Edgeworth, F. H. 1935. 'The cranial muscles of vertebrates. Cambridge Univ. Press, Cambridge,

Enzow, D. H. 1963. Principles of bone remodeling. Charles C. Thomas, Springficld, Illinois.

Enlow, D. H. 1969. 'The bones of Reptiles. In Gans, C., A. d'A. Bellairs, and 'T. S. Parsons (eds.), Biology of the Reptilia. Vol. I (Morphology A). Academic Press, London, pp. 45-81.

Estes, R., K. de Queiroz, And J. Gauthier. 1988. Phylogenetic relationships within Squamata. In Estes, R. and G. K. Pregill (eds.). The phylogenetic relationships of the lizard familics: cssays commemorating Charles L. Camp. Stanford Univ. Press, Palo Alto, pp. 119-281.

Farner, D. S. 1960. Digestion and the digestive system. In Marshall, H. J. (ed.), Biology and comparative physiology of birds. Vol. 1. Academic Press, New York, pp. 411-467.

FarRis, J. S. 1970. Methods for computing Wagner trees. Syst. Zool. 19: 83-92.

FARRIS, J. S. 1982. Outgroups and parsimony. Syst. Zool. 31: 328-334. 
FINK, W. L. 1982. The conceptual relationship between ontogeny and phylogeny. Paleobiology 8: 254-264.

Forey, P. L. 1986. Relationships of lungfishes. J. Morph. (Supplement) I: 75-91.

Foske, H. 1934. Das Geruchsorgan von Xenopus laevis. Zeit. Anat. Entwickl. 103: 519-550.

Fox, H. 1976. The urinogenital system. In Gans, C., and T. S. Parsons (eds.), Biology of the Reptilia. Vol. 6 (Morphology E). Academic Press, London, pp. 1-157.

Foxon, G. E. H. 1955. Problems of the double circulation in vertebrates. Biol. Rev. Cambridge Philos. Soc. 30: 196-228.

Francis, E. T. B. 1934. The anatomy of the salamander. Oxford Univ. Press, Oxford.

Frank, G. H. 1954. The development of the chondrocranium of the ostrich. Annals Univ. Stellenbosch, 30(A): 179-247.

Fuchs, H. 1907. Untersuchungen über Ontogenie und Phylogenie der Gaumenbildungen bei den Wirbeltieren. Erste Mitteilung. Über den Gaumen der Schildkroten und seine Entwicklungsgeschichte. Zeit. Morph. Anthrop. 10: 409-463.

Fuchs, H. 1915. Uber den Bau und die Entwicklung des Schadels der Chelone imbricata. In Voeltzkow, A. (ed.), Reise in Ostafrika in den Jahren 1903-1905, Wissenschaftliche Ergebnisse. Vol. 5. E. Schweizerbart, Stuttgart, pp. 1-325.

Fullarton, M. H. 1933. On the development of the olfactory organ in Protopterus. Proc. Roy. Soc. Edinburgh 53: 1-6.

Gabe, M. 1970. The adrenal. In Gans, C., and T. S. Parsons (eds.), Biology of the Reptilia. Vol. 3 (Morphology C). Academic Press, London, pp. 263-318.

GafFney, E. 1975. A phylogeny and classification of the higher categories of turtles. Bull. Amer. Mus. Nat. Hist. 155: 391-436.

Gaffney, E. 1979. Tetrapod monophyly: a phylogenetic analysis. Bull. Carnegie Mus. Natur. Hist. 13: 92-105.

Gaffney, E. 1980. Phylogenetic relationships of the major groups of amniotes. In Panchen, A. L. (ed.), The terrestrial environment and the origin of land vertebrates. Academic Press, London, pp. 593-610.

Gaffney, E., and M. MaKenna. 1979. A Late Permian captorhinid from Rhodesia. Amer. Mus. Novit. (2688): 1-15.

Gardiner, B. 1982. Tetrapod classification. Zool. J. Linnean Soc. 74: 207-232.

Gauthier, J. 1984. A cladistic analysis of the higher systematic categories of the Diapsida. Unpubl. Ph.D. dissertation, Univ. California, Berkeley.

Gauthier, J. 1986. Saurischian monophyly and the origin of birds. California Acad. Sci. Mem. 8: $1-56$.

Gauthier, J., R. Estes, and K. de Queiroz. 1988a. Lepidosauromorpha. In Estes, R., and G. K. Pregill (eds.), The phylogenetic relationships of the lizard families: essays commemorating Charles L. Camp. Stanford Univ. Press, Palo Alto. pp. 15-98.

Gauthier, J., A. G. Kluge, and T. Rowe. 1988b. The early evolution of Amniota. In Benton, M. J. (ed.), The Phylogeny and Classification of the Tetrapods. Vol.. 1. Amphibians, Reptiles and Birds. Systematics Association Special Volume 35A, Clarendon Press, Oxford, pp. 103-155.

Gegenbaur, C. 1873. Über die Nasenmuscheln der Vogel. Jena. Ziet. Naturw. 7: 1-21.

Goonrich, E. S. 1916. On the classification of the Reptilia. Proc. Roy. Soc. London 89(B): 261-276.

Goodrich, E. S. 1930. Studies on the structure and development of vertebrates. Vol. 2. Dover Public., New York. (1958 reprint.)

Gould, S. J. 1986. The Archaeopteryx flap. Nat. Hist. Mag. 9: 16.

Gregory, W. K. 1947. Monotremes and the palimpsest theory. Bull. Amer. Mus. Nat. Hist. 88: $1-52$.

Grewe, F. J. 1951. Nuwe gegewens aangaande die ontogenese van die neuskliere, die orgaan van Jacobson en die dekbene van die skedel by die genus:Anas. Ann. Univ. Stellenbosch 27: $69-99$.

Griffiths, M. 1978. The biology of the monotremes. Academic Press, New York.

Hadley, M. E., and W. C. Quevado. 1966. Vertebrate epidermal-melanin unit. Nature 209: 1334-1335.

Haines, R. W. 1942. The tetrapod knee joint. J. Anat. 76: 270-301. 
Haines, R. W. 1969. Epiphyses and sesamoids. In Gans, C., A. d'A. Bellairs, and T. S. Parsons (eds.), Biology of the Reptilia. Vol. 1 (Morphology A). Academic Press, London, pp. 81-115.

HaLL, B. K. 1984. Developmental processes underlying the evolution of cartilage and bone. In Ferguson, M. W. J. (ed.), The structure, development and evolution of reptiles. Zool. Soc. London Symp. No. 52. Academic Press, London, pp. 155-176.

Heaton, M.J. 1980. The Cotylosauria: A reconsideration of a group of archaic tetrapods. In Panchen, A. L. (ed.), The terrestrial environment and the origin of land vertebrates. Academic Press, London, pp. 497-551.

Heilmann, G. 1927. The origin of birds. Witherby, London.

Hendy, M. D., AND D. PENNy. 1982. Branch and bound algorithms to determine minimal cvolutionary trecs. Math Biosc. 59: 277-290.

Hennig, W. 1966. Phylogenetic systematics. Univ. Illinois Press, Urbana.

Hennig, W. 1981. Insect phylogeny. John Wiley, New York.

Hildebrand, M. 1974. Analysis of vertebrate structure. John Wilcy, New York.

Hil.., C. R., And J. M. Camus. 1986a. Evolutionary cladistics of Marattialean ferns. Bull. Br. Mus. Nat. Hist. (Bot.) 14: 219-300.

Hil.t, C. R., and J. M. Camus. 1986b. Pattern cladistics or evolutionary cladistics? Cladistics 2: $362-375$.

HiLL, J. P. 1933. V. The development of the Monotremata. Part II. The structure of the egg-shell. Trans. Zool. Soc. London 24: 443-456.

Hili., J. P., And G. DE BeER. 1949. The development and structure of the egg-tooth and caruncle in the monotremes and on the occurrence of vestiges of the egg-tooth and caruncle in marsupials. 'Irans. Zool. Soc. London 26: 503-544.

Hinsburg, V. 1901. Die Entwicklung der Nasenhohle dei Amphibien. Theil I und II: Anuren und Urodelen. Arch. Mikr. Anat. Entwickl. 58: 411-482.

Holmes, E. B. 1975a. A reconsideration of the phylogeny of the tetrapod heart. J. Morph. 147: $209-228$.

Holmes, E. B. 1975b. Manual of comparative anatomy. Macmillan, New York.

Hopson, J. A. 1964. The braincase of the advanced mammal-like reptile, Bienotherium. Postilla $(87): 1-30$.

Hopson, J. A. 1979. Paleoneurology. In Gans, G., R. G. Northcutt, and P. Ulinski (cds.), Biology of the Reptilia. Vol. 9 (Neurology A). Academic Press, London, pp. 39-146.

Hopson, J. A. 1980. Relative brain size in dinosaurs: implications for dinosaurian endothermy. In Thomas, R. D. K., and E. C. Olson (eds.), A cold look at the warm blooded dinosaurs. No. 28. Amer. Assoc. Advan. Sci. Selected Symposium. pp. 287-310.

Huene, F. F. von. 1946. Die grossen stamme der Tetrapoden in den geologischen Zeiten. Biologischen Zentralblatt 65: 266-275.

Huettner, A. F. 1966. Fundamentals of comparative embryology of the vertebrates. Macmillan, New York.

Huxley, T. H. 1864. Lectures on the elements of comparative anatomy. J. Churchill, London.

HuXley, 'I'. H. 1869. On the representatives of the malleus and the incus of the Mammalia in the other Vertcbrata. Proc. Zool. Soc. London 1869: 391-407.

Huxley, T. H. 1871. A manual of the anatomy of vertebrated animals. J. \& A. Churchill, London.

Iordansky, N. N. 1973. The skull of the Crocodilia. In Gans, C., and T. S. Parsons (eds.), The Biology of the Reptilia. Vol. 4 (Morphology D). Academic Press, London, pp. $201-262$.

JefFerles, R. P. S. 1979. The origin of chordates a methodological essay. In House, M. R. (ed.), The origin of major invertebrate groups. Syst. Assoc. Spec. Vol. 12. London, pp. 443-447.

Jefferies, R. P. S. 1986. Ancestry of the vertebrates. Cambridge Univ. Press, New York.

Jollie, M. 1962. Chordate morphology. Reinhold, New York.

Jordan, T. W., J. N. Smith, and L. Vaughan. 1980. Benzoic acid conjugation in Tuatara. Biochem. Syst. Ecol. 8: 101-103.

Jurgens, J. D. 1971. The morphology of the nasal region of Amphibia and its bearing on the phylogeny of the group. Ann. Univ. Stellenbosch 46: 1-146.

Kamal, A. M. 197I. On the fissura metotica in Squamata. Bull. Zool. Soc. Egypt 23: 53-57. 
Kashyap, H. V. 1960. The reptilian heart. Proc. Natn. Inst. Sci. India 26(B): 234-251.

Kemp, T. S. 1982. Mammal-like reptiles and the origin of mammals. Academic Press, London.

Kennedy, J. P., And H. L. Brockman. 1965. Open heart surgery in Alligator mississipiensis Daudin. Herpetologica 21: 6-15.

Kielan-Jaworowska, Z., R. Presley, and C. Poplin. 1986. The cranial vascular system in taeniolabidoid multituberculate mammals. Phil. Trans. Roy. Soc. London 313(B): $525-602$.

KING, A. S. 1979. Systema respiratorium. In Baumel, J. J., A. S. King, A. M. Lucas, J. E. Breazile, and H. E. Evans (eds.), Nomina anatomica avium. Academic Press, London, pp. $227-265$.

KLuGE, A. G. 1985. Ontogeny and phylogenetic systematics. Cladistics 1: 13-27.

KLuge, A. G. 1988. The characterization of ontogeny. In Humphries, C. J. (ed.), Ontogeny and systematics. Columbia Univ. Press, New York, pp. 57-81.

KLUGe, A. G., AND J. S. FARris. 1969. Quantitative phyletics and the evolution of anurans. Syst. Zool. 18: 1-32.

Kluge, A. G., and R. E. Strauss. 1985. Ontogeny and systematics. Ann. Rev. Ecol. Syst. 16: 247-268.

Kluger, M. 1979. Fever in ectotherms: evolutionary implications. Amer. Zool. 19: 295-304.

Kobayash1, H., AND M. WADA. 1973. Neuroendocrinology in birds. In Farner, D. S., J. R. King, and K. C. Parlas (eds.), Avian biology. Vol. 3. Academic Press, London, pp. 287-347.

Kunn, H.-J. 1971. Die Entwicklung und Morphologie des Schadels von Tachyglossus aculeatus. Abh. senckenb. naturforsch. Ges. 528: 1-192.

Kuhn, H.-J., and U. Zeller. 1987. The cavum epiptericum in monotremes and therian mammals. In Kuhn, H.-J., and U. Zeller (eds.), Morphogenesis of the mammalian skull. Verlag Paul Parey, Hamburg, pp. 51-70.

Kunn-Schnyder, E. 1987. Bemerkungen eines Palaontologen zur Klassifikation der Amniota von B. G. Gardiner (1982) und S. Løvtrup (1985). Z. zool. Syst. Evolut.-forsch. 25: 1-8.

LAwson, R. 1970. The caecilian cardio-vascular system and intra-amphibian relationships. J. Zool. London 160: 199-229.

Lawson, R. 1979. The comparative anatomy of the circulatory system. In Wake, M. H. (ed.), Hyman's Comparative Vertebrate Anatomy. 3rd edn. Univ. Chicago Press, Chicago, pp. 448-554.

LEGHISSA, S. 1962. L'evoluzione del tetto ottico nei bassi vertebrati. Arch. Ital. Anat. Embriol. 67: 344.

Løvtrup, S. 1977. The phylogeny of Vertebrata. John Wiley, London.

Løvtrup, S. 1985. On the classification of the taxon Tetrapoda. Syst. Zool. 34: 463-470.

LupPa, H. 1977. Histology of the digestive tract. In Gans, C., and T. S. Parsons (eds.), Biology of the Reptilia. Vol. 6 (Morphology E). Academic Press, London, pp. 225-313.

Maddison, W. P., M. J. Donoghue, and D. R. Maddison. 1984. Outgroup analysis and parsimony. Syst. Zool. 33: 83-103.

Maderson, P. F. A. 1972. When? Why? and How?: Some speculations on the evolution of the vertebrate integument. Am. Zool. 12: 159-171.

Maderson, P. F. A., And R. H. Sawyer. 1979. Scale embryogenesis in birds and reptiles. Anat. Rec. 193: 609.

MAIER, W. 1987. The ontogenetic development of the orbitotemporal region in the skull of Monodelphis domestica (Didelphidae, Marsupialia), and the problem of the mammalian alisphenoid. In Kuhn, H.-J., and U. Zeller (eds.), Morphogenesis of the mammalian skull. Verlag Paul Parey, Hamburg, pp. 71-90.

MaIsey, J. G. 1987. Notes on the structure and phylogeny of vertebrate otoliths. Copeia (2). 495-499.

Manner, H. W. 1964. Elements of comparative vertebrate embryology. Macmillan, New York.

Marshall, L. G. 1979. Evolution of metatherian and eutherian (mammalian) characters: a review based on cladistic methodology. Zool. J. Linnean Soc. 66: 369-410.

Martin, L. 1983. The origin of birds and of avian flight. In Johnston, R. (ed.), Current Ornithology I, pp. 105-129.

Matson, R. H. 1985. "Ldh-X": Is it an avian character? Abstracts 103rd State Mecting of the Américan Ornithologists' Union. Arizona State Univ., 'Tempe, Arizona. Abstract No. 169. 
Matthes, E. 1934. Geruchsorgan. In Bolk, L., E. Goppert, E. Kallius, and W. Lubosch (eds.), Handbuch der vergleichenden Anatomie der Wirbeltiere. Vol. 2. Urban and Schwarzenberg, Berlin and Wien, pp. 879-948.

MaCotter, R. E. 1917. The vomero-nasal apparatus in Chrysemys punctata and Rana catesbiana. Anat. Rec. 13: 51-67.

MaFarlane, R. W. 1963. The taxonomic significance of avian sperm. Proc. XIII Internat. Orn. Congr., Ithaca 1962: 91-102.

Mclelland, J. 1979. Systema digestorum. In Baumel, J. J., A. S. King, A. M. Lucas, J. E. Brcazile, and H. E. Evans (eds.), Nomina anatomica avium. Academic Press, London, pp. $267-287$.

Mikhalovics, V. von. 1898. Nasenhohle und Jacobsonsches Organ. Eine morphologische Studie. Arb. Anat. Inst., Wiesbaden (Anat. Hefte, 1) 11: 1-107.

Miles, R. S. 1977. Dipnoan (lungfish) skulls and the relationships of the group: a study based on new species from the Devonian of Australia. Zool. J. Linnean Soc. 61: 1-328.

Miller, J. 1976. Biochemical characteristics and biological significance of the genetically distinct collagens. Molec. Cell. Biochem. 13: 165-192.

Miller, M. R., and M. D. Lagios. 1970. The pancreas. In Gans, C., and T. S. Parsons (eds.), The Biology of the Reptilia. Vol. 3 (Morphology G). Academic Prcss, London, pp. 319-346.

Moore, W. J. 1981. The mammalian skull. Cambridge Univ. Press, Cambridge.

NeILL, W. 'T. 1971. The last of the ruling reptiles: alligators, crocodiles, and their kin. Columbia Univ. Press, New York.

Nelsen, O. E. 1953. Comparative embryology of the vertebrates. Blakiston, New York.

Nick, L. 1912. Das Kopfskelet von Dermochelys coriacea L. Zool. Jahrb., Abt. Anat. 33: 1-238.

Nishimura, H. 1980. Evolution of the renin-angiotensin system. In Pang, P. K. T., and A. Epple (eds.), Evolution of vertebrate endocrine systems. Texas Tech. Press, Lubbock, pp. $373-404$.

Northcutr, R. G. 1981. Evolution of the telencephalon in nonmammals. Ann. Rev. Neurosci. 4: $301-350$.

Nortнсuтt, R. G. 1984. Anatomical organization of the optic tectum in reptiles. In Vanegas, H. (cd.), Comparative neurology of the optic tectum. Plenum Press, New York, pp. $547-600$.

Northcutr, R. G. 1986. Lungfish neural characters and their bearing on Sarcopterygian phylogeny. Jour. Morph., Supplement (1): 277-297.

Novacex, M.J., AND M. A. Norell. 1982. Fossils, phylogeny, and taxonomic rates of evolution. Syst. Zool. 31: 366-375.

O'Donoghue, C. H. 1921. The blood vascular system of the tuatara, Sphenodon punctatus. Philos. Trans. Roy. Soc. London 210(B): 175-252.

O'Flaherty, J.J. 1970. A golgi analysis of the optic tectum of the mallard duck. J. Hirnforsch. 12: 389.

Osawa, G. 1897. Beitrage zur Lehre von den Eingeweiden der Hatteria punctata. Arch. Mikrosk. Anat. EntwMech. 49: 113-226.

Osborn, H. F. 1903. The reptilian subclasses Diapsida and Synapsida and the carly history of the Diaptosaura. Mem. Amer. Mus. Nat. Hist. 1: 449-508.

Osborn, J. W. 1984. From reptile to mammal: evolutionary considerations of the dentition with cmphasis on tooth attachment. In Ferguson, M. W.J. (ed.), The structure, development and evolution of reptiles. No. 52. Symp. Zool. Soc. London, pp. 549-574.

Oster, G., And P. Alberch, 1982. Evolution and bifurcation of developmental programs. Evolution 36: 444-459.

OWEn, R. 1866. On the anatomy of vertebrates. Vols. I and II. Longmans, Green and Co., London.

OWEn, R. 1868. On the anatomy of Vertebrates. Vol. III. Longmans, Green and Co., London.

Packard, G. G., C. R. Tracy, and J. J. Roth. 1977. 'The physiological ecology of reptilian eggs and embryos, and the evolution of viviparity within the class Reptilia. Biol. Rev. 52: $1-105$.

PAnchen, A. L. 1980. The origin and relationships of the anthracosaur Amphibia from the late Palaeozoic. In Panchen, A. L. (ed.), The terrestrial environment and the origin of land vertcbrates. Academic Press, London, pp. 319-350. 
Parsons, T. S. 1959. Studies on the comparative embryology of the reptilian nose. Bull. Mus. Comp. Zool., Harvard 120: 101-277.

Parsons, T. S. 1967. Evolution of the nasal structure in the lower tetrapods. Amer. Zool. 7 : $397-413$.

Parsons, 'T. S. 1970. The nose. In Gans, C., and T. S. Parsons (eds.), Biology of the Reptilia. Vol. 2 (Morphology B). Academic Press, London, pp. 99-191.

Patten, B. M. 1952. Embryology of the pig. 3rd edn. Blakiston, New York.

Patterson, C. 1977a. The contribution of paleontology to teleostean phylogeny. In Hecht, M. K., P. C. Goody, and B. M. Hecht (eds.), Major patterns in vertebrate evolution. Plenum Press, New York, pp. 579-643.

Patterson, C. 1977b. Cartilage bones, dermal bones and membrane bones, or the exoskeleton versus the endoskcleton. In Andrews, S. M., R. S. Miles, and A. D. Walker (cds.), Problems in vertcbrate evolution. Ser. 4. Linnean Soc. Symp., pp. 77-121.

Patterson, C. 1981a. Significance of fossils in determining evolutionary relationships. Ann. Rev. Ecol. Syst. 12: 195-223.

Patterson, C. 1981b. Agassiz, Darwin, Huxley, and the fossil record of teleost fishes. Bull. Br. Mus. Nat. Hist. (Geol.) 35: 213-224.

Patrerson, C. 1982. Morphological characters and homology. In Joysey, K. A., and A. E. Friday (eds.), Problems of phylogenetic reconstruction. Academic Press, New York, pp. 21-74.

Patterson, C., and D. E. Rosen. 1977. Review of ichthyodectiform and other Mesozoic teleost fishes and the theory and practice of classifying fossils. Bull. Am. Mus. Nat. Hist. 158: $81-172$.

Pearson, A. K. 1985. Development of the pituitary in reptiles. In Gans, C., F. Billett, and P. F. A. Maderson (eds.), Biology of the Reptilia. Vol. I4 (Development A). John Wiley, New York, pp. 679-719.

Platnick, N. I. 1986a. "Evolutionary cladistics" or cvolutionary systematics? A review of "Evolutionary cladistics of Marattialean ferns." By C. R. Hill and J. M. Camus. Cladistics 2: 288-296.

Platnick, N. I. 1986b. Taxonomic methods and "evolutionary cladistics." Cladistics 2: $375-377$.

Porter, K. R. 1972. Herpetology. Saunders, Philadelphia.

Presley, R. 1981. Alisphenoid equivalents in placentals, marsupials, monotremes and fossils. Nature 294: 668-670.

Prestey, R., and F. L. D. Steer.. 1978. 'The pterygoid and ectopterygoid in mammals. Anat. Embryol. 154: 95-110.

Pritchard, P. C. H., 1979. Encyclopedia of turtles. T. F. H. Publ., New Jersey.

Pumphrey, R. J. 1961. Part II. Sensory organs: hearing. In Marshall, A. J. (ed.), Biology and comparative physiology of birds. Academic Press, New York, pp. 69-86.

Quay, W. B. 1979. The parictal eye-pincal complex. In Gans, G., R. G. Northcutt, and P. Ulinski (eds.), Biology of the Reptilia. Vol. 9 (Neurology A). Academic Press, New York, pp. 245-406.

Quevado, W. C. 1972. Epidermal melanin units: melanocyte-keratinocyte interactions. Amer. Zool. 12: 35-41.

RaFN, S., and K. G. Wingstrand. 1981. Structure of intestine, pancreas, and splecn of the Australian lungfish, Neoceratodus forsteri (Krefft). Zool. Scripta 10: 223-239.

Ramaswami, L. S. 1944. An account of the heart and associated structures in some gencra of Apoda. Proc. Zool. Soc. London 114: 117-138.

Raynaud, A., and C. Pieau. 1985. Embryonic development of the genital system. In Gans, C., and F. Billett (eds.), Biology of the Reptilia. Vol. 15 (Development B). John Wiley, New York, pp. 149-300.

Reisz, R. R. 1972. Pelycosaurian reptiles from the Middle Pennsylvanian of North America. Bull. Mus. Comp. Zool., Harvard 144: 27-62.

RIEPPEt, O. 1980. Homology, a deductive concept? Z. zool. Syst. Evolut.-forsch. 18: 315-319.

RIEPPEL, O. 1985. The recessus scalae tympani and its bearing on the classification of reptiles. J. Herpet. 19: 373-384.

Robertson, J. I. 1913. "devel. Lepidosiren paradoxa interventricular septum." Quart. J. Microsc. Sci., 59: 53-132. 
Romer, A. S. 1956. Osteology of the reptiles. Univ. Chicago Press, Chicago.

Romer, A. S. 1966. Vertebrate paleontology. Univ. Chicago Press, Chicago.

Rosen, D. E., P. L. Forey, B. G. Gardiner, and C. Patterson. 1981. Lungfishes, tetrapods, paleontology and plesiomorphy. Bull. Amer. Mus. Natur. Hist. 167: 159-267.

Rowe, T. 1986a. Osteological diagnosis of Mammalia, L. 1758, and its relationship to extinct Synapsida. Ph.D. dissertation, Univ. California, Berkeley.

Rowe, T. 1986b. Homology and evolution of the deep dorsal thigh musculature in birds and other Reptilia. J. Morph. 189: 327-346.

Rowe, T. 1988. Definition, diagnosis and origin of Mammalia. J. Vert. Paleo. in press.

Rudebeck, B. 1945. Contributions to forebrain morphology in Dipnoi. Acta Zool. Stockholm, 26: 9-156.

SADLER, P. M. 1981. Sediment accumulation rates and the completeness of stratigraphic sections. J. Geol. 89: 569-584.

Sadler, P. M., And L. W. Dingus. 1982. Expected completeness of sedimentary sections: estimating a time-scale dependent, limiting factor in the resolution of the fossil record. Proc. Third N. Amer. Paleo. Conv. 2: $46 \mathrm{l}-464$.

Sarasin, P., And F. Sarasin. 1890. Blindwuhle Ichthyophis glutinosus, L. Ergebnisse Naturwissenschaftlicher Forschungen auf Ceylon. Weisbaden 2: 1-263.

Schmaulhausen, I. I. 1968. The origin of terrestrial vertebrates. Academic Press, New York.

Schmidt-Nielsen, K. 1979. Animal physiology: adaptation and environment. Cambridge Univ. Press, Cambridge.

Schuch, K. 1934. Das Geruchsorgan von Triton alpestris. Zool. Jahrb., Abt. Anat. 59: 69-134.

Schultze, H.-P. 1986. Dipnoans as sarcopterygians. J. Morph. (Supplement) 1: 39-74.

Schwab, M. E. 1979. Variation in the rhombencephalon. In Gans, C., R. G. Northcutt, and P. Ulinski (eds.), Biology of the Reptilia. Vol. 10 (Neurology B). Academic Press, London, pp. 201-246.

Seydel, O. 1895. Uber die Nasenhohle und das Jacobson'sche Organ der Amphibien. Morph. Jahrb. 23: 453-543.

Seydel, O. 1896. Über die nasenhohle und das Jacobson'sche Organ der Land- und Sumpfschildkroten. Festschrift 70 Geburstag Gegenbaur 2: 385-486.

Shaner, R. F. 1962. Comparative development of the bulbus and ventricles of the vertebrate heart with special reference to Spitzer's theory of heart malformations. Anat. Rec. 142: 519-529.

Shaw, G., E. Debus, and K. Weber. 1984. The immunological relatedness of neurofilament proteins of higher vertebrates. Eur. J. Cell Biol. 34: 130-136.

Shindo, T. 1915. Über die Bedeutung des Sinus cavernosus der Sauger mit vergleichend anatomisher Berucksichtigung anderer Kopfvenen. Anat. Hefte 1: 319-495.

Simons, J. R. 1965. The heart of the tuatara, Sphenodon punctatus. J. Zool. London 146: 451-466.

Smith, H. M. 1960. Evolution of chordate structure. Holt, Rinehart and Winston, New York.

Sperber, I. 1960. Excretion. In Marshall, A. J. (ed.), Biology and comparative physiology of birds. Vol. 1. Academic Press, New York, pp. 469-492.

Stark, D. 1979. Cranio-cerebral relations in Recent reptiles. In Gans, C., R. G. Northcutt, and P. Ulinski (eds.), Biology of the Reptilia. Vol. 9 (Neurology A). Academic Press, London, pp. 1-38.

Stebbins, R. C. 1948. Nasal structure in lizards with reference to olfaction and conditioning of the inspired air. Amer. J. Anat. 83: 183-222.

Stevens, P. F. 1984. Homology and phylogeny: morphology and systematics. Syst. Bot. 9: 395-409.

Swofford, D. L. 1984. PAUP: Phylogenetic Analysis Using Parsimony. Version 2.3. Privately printed documentation. Illinois Natural History Survey, Champaign, Illinois.

Tarsitano, S., and M. K. Нecht. 1980. A reconsideration of the reptilian relationships of Archaeopteryx. Zool. J. Linnean Soc. London 69: 149-182.

Tonutti, E. 1931. Beitrag zur Kenntnis der Gymnophionen XV. Das Genitalsystem. Morph. Jahrb. 68: 151-292.

Tonutri, E. 1932. Vergleichend-morphologische Studie über die Phylogenie des Enddarmes und des Kopulationsorganes der mannlichen Amnioten, ausgehend von den Gymnophionen. Morph. Jahrb. 70: 101-130. 
TonutT1, E. 1933. Beitrag zur Kenntnis der Gymnophionen XIX. Untersuchungen der Kopulationsorgane bei weiteren Gymnophionenarten. Morph. Jahrb. 72: 155-210.

Turner, S. C. 1967. Development of the heart in a newt and a frog. Acta Zoologica, XL.VIII: $1-15$.

ULINSKI, P. S. 1983. Dorsal ventricular ridge: a treatise on forebrain organization in reptiles and birds. John Wiley, New York.

Underwoon, G. 1970. The eye. In Gans, C., and T. S. Parsons (eds.), Biology of the Reptilia. Vol. 2 (Morphology B). Academic Press, New York, pp. 1-97.

VAn Andel, T. H. 1981. Consider the incompleteness of the geological record. Nature 294: 397-398.

Van Gelderen, C. 1924. Dic Morphologie der Sinus durae matris. Zweiter Teil. Die vergleichende Ontogenie der neurocraniellen Venen der Vogel und Saugetiere. Zeit. Anat. 74: 432-508.

Van Gelderen, C. 1925. Die Morphologie der Sinus durae matris. Dritter Teil. Vergleichendes, Erganzendes, Phyletisches und Zusammenfassendes übcr die neurocraniellen Venen der Vertebraten. Zeit. Anat. 75: 525-596.

VAn Tyne, T., AND A. J. Berger. 1976. Fundamentals of ornithology. 2nd edn. John Wiley, New York.

Voeltzkow, A. 1899. Beitrage zur Entwicklungsgeschichte der Reptilien. Biologie und Entwicklung der ausseren Korperform von Crocodylus madagascariensis Grand. Abh. Senckenb. Naturforsch. Ges. 26: 1-150.

WAKE, M. H. 1972. Evolutionary morphology of the caecilian urogenital system. IV. The cloaca. J. Morph. 136: 353-366.

WALKer, A. 1985. The braincase of Archaeopteryx. In Hecht, M. K., J. H. Ostrom, G. Viohl, and P. Wellnhofer (eds.), The beginning of birds: proceedings of the international Archaeopteryx conference, Eichstatt 1984. Freunde des Jura-Museums Eichstatt, Willibaldsburg, Eichstatt, pp. 123-134.

Walls, G. L. 1942. The vertebrate eye and its adaptive radiation. Cranbrook Inst. Sci. Bull. 9: $1-785$

Werner, C. F. 1960. Das Gehörorgan der Wirbeltiere und des Menschen. Beispel für eine verglcichende Morphologie der Lagesbezichungen. Thieme, Leipzig.

Wever, E. G. 1978. The reptile ear: its structure and function. Princeton Univ. Press, New Jersey.

Wiedersheim, R. 1897. Elements of the comparative anatomy of vertebrates. 2nd edn (Founded on 3rd German edn) Macmillan, London.

Willmann, R. 1987. Phylogenetic systematics, classification and the plesion concept. Verh. naturwiss. Ver. Hamburg 29: 221-233.

Wingstrand, K. G. 1951. The structure and development of the avian pituitary. Gleerup, Lund.

Woodward, A. S. 1898. Outlines of vertebrate paleontology for students of zoology. Cambridge, England.

WYld, J. A., AND A. H. BRush. 1979. The molecular heterogencity and diversity of reptilian keratins. J. Molec. Evol. 12: 331-347.

Wyld, J. A., AND A. H. BRush. 1983. Keratin diversity in the reptilian epidermis. J. Exp. Zool. 225: 387-396.

Zeller, U. 1985. The morphogenesis of the fenestra rotunda in mammals. In Duncker, H.-R., and W. Fleischer (eds.), Vertebratc Morphology Fortschritte der Zoologie. Vol. 30. Gustav Fischer Vcrlag, Stuttgart, pp. 153-157.

Ziswiler, V., AND D. S. Farner. 1972. Digestion and the digestive system. In Farner, D. S., J. R. King, and K. C. Parkes (eds.). Avian biology. Academic Press, New York, pp. 343-430.

Zuckerkande, E. 1910. Uber die Wechselbeziehung in der Ausbildung des Jacobsonsche Organ der Amphibien. Anat. Hefte 1 41: 1-75.

\section{Appendix A}

The diagnostic information for the nodes and terminal taxa in Fig. 3 are summarized below. The taxonomic names we recognize are placed in parentheses at their nodes. The characters without a letter prefix (1-207) refer to those listed in Table 2; the remaining 
characters, with a prefix, are from Table 1. We place synapomorphies in the most parsimonious phylogeny at the level at which observation confirms that they are diagnostic. Due to missing data, some characters may have originated earlier than indicated. For example, a complete interventricular septum is diagnostic of the heart of those amniotes in which the apomorphic condition can be observed, namely, mammals and archosaurs. We recognize that it is possible that this synapomorphy arose at earlier points in the phylogeny of Synapsida on the one hand, and Archosauromorpha on the other. Accordingly, the one or more nodes to which the state-change might apply are placed in brackets. Reversals to the ancestral state are preceded by a negative sign. Equivocal characters are followed by one or two asterisks. One asterisk indicates that additional evidence discussed in the text, or in Gauthier (1984) or Rowe (1986a), has been used to eliminate some or all of that ambiguity. Two asterisks indicate that the ambiguity observed in Table 2 and Fig. 3 remains, and these characters are placed at all nodes at which they could have originated. All characters refer to state 1 , unless stated otherwise in parentheses.

Node 1 (Amniota): G21**, G24a*, G30, G32, G34b, G34b**, G34c**, G37(1 or 2)**, L10, L13, L15(2), L15a(2), L15b(2), L18, L19, 6, 148*.

Node 2 (Reptilia): G5c, G34**, G34a*[1], G34b(2)*, G35a, G37**, L16a, A6, Al1, $\Lambda 15, \Lambda 17, \Lambda 19, \Lambda 29,24,26^{*}, 39,41,56^{*}, 57^{*}, 74,100,122^{*}, 131,139 * *$, 200.

Node 3 (Diapsida): 15, 35, 36, 37*, 38*, 57(2)*, 82, 83, 139**, 159, 195, 202.

Node 4 (Sauria): G21**[3], G34c**[3], L7(1 or 2)**[3], A9[3], A14*[3], A20[3], $\mathrm{A} 25[3], 20,33,62,69,104,111,132,142,147,-148^{*}, 149,153,154,169,170,171$, $175,176,177,184,189,190,201,204$.

Node 5 (Archosauromorpha): 1, 2, 17, 25, 34, 50, 52, 68, 70, 78, 124, 151, 164, 196* , 198, $205,206$.

Node 6: $16^{* *}, 48,143$.

Node 7: 4,47 .

Node 8: 16**, 109.

Node 9 (Archosauriformes): 10, 22[5-8], 31, 35(2), 36(2), 71, 108, 110, 193.

Node 10: 23, 41(2) [9], 77, 87, 106, 107, 119, 123, 127, 128, 146, 162, 199, 203.

Node 11: 16, 129**, 160, 167, 168, 184(2), 186, 187, 191.

Node 12 (Archosauria): G3[5-11], G3a[5-11], G7*[5-11], G8a[5-11], G12a[5-11], G20[5-11], G22a[5-11], G25[5-11], G27a[5-11], G27b[5-11], G34**[5-11], G34d[5-11], G37(3)[5-11], L1a[5-11], L6a[5-11], L7**[5-11], L8[5-11], L9[5-11], A7[5-11], A8[5-11], A10[5-11], A12[5-11], A15(2)[5-11], A16[5-11], $\Lambda 18[5-11], 32,80,126,129 * *, 144,155[11], 198(2)[10-11]$.

Nade 13 (Anapsida): 25, 55, 57**, 151.

Node 14 (Synapsida): $4,22,37^{*}, 38 *, 70,75,98,130,141^{* *}$.

Node 15: 14[14], 15**, 30, 40, 99, 171 .

Node 16: 8, 51, 92, 141**, 169 .

Node 17 (Sphenacodontia): 15**, 33, 79, 102, 104, 105, 108, 110, 112, 113[16], 116[16], $119,120,139$.

Node 18 (Therapsida): 3, 18, 27, 42, 49, 69, -70, 100, 101, 114, 122, 124, 127, 142, 146, $147,152,157,166,177,178,179,184(2), 186,187,188,189,190,194,200^{* *}$, $206^{* *}, 207$.

Node 19: 11, 21, 24, 38(2), 60, 128, 163, 167. 
Node 20: 23, 59, 66, 95, 136, 137, 200**, 206**.

Node 21: $-8,10,15(2), 46,87,121,123 * *, 131,133,135,153,161,165,172,182^{* *}$, $188(2)$.

Node 22 (Eutheriodontia): 13, - 14, 17, - 18, 19, 20, 53, 73, 151.

Node 23 (Cynodontia) : 7, 9, - 27, 43, 61, 65, 67, 81, 82, 83(2), 84, 86, 88, 101(2), - 108, $115,123 * *, 145,182 * *$

Node 24: 29, 34, 44, 54, 85, 89, 97, 125**.

Node 25: 49(2), 63, 90, 93, 96, 103, 106, 107, 117**, 125**.

Node 26: 16, 28, $-30^{* *}, 55,-125^{* *}, 129,150,156,173,180,181$.

Node 27 (Mammaliamorpha): 2, 5, 12, 45, 64, 76, - 79, 94*, - 117**, 118, 134, 138, $158,-163,-172,174,183,185,188(3), 192,197,198(2)$.

Node 28 (Mammaliaformes): 25, - 30**, 72, 91, 125**, 140.

Araeoscelidia: 126.

Biarmosuchia: 200**, 206**.

Captorhinidae: 104, 126.

Casea: $104 * *, 141 * *, 163 * *$.

Choristodera: $16^{* *}, 23,38(2), 54,58,127,128,167,199$.

Diademodon: $117 * *, 125 * *$.

Dicynodontia: $123 * *, 182 * *$.

Dinocephalia: $-200 * *$.

Edaphosaurus: $-4,-15^{* *}, 104,111,163$.

Erythrosuchidae: 121 .

Exaeretodon: $-30 * *, 117 * *,-125 * *$.

Gorgonopsia: 43.

Lepidosauromorpha: (these are synapomorphies of Lepidosauria that may have arisen earlier in Lepidosauromorpha) - G13, - G30, - G32, - G34**, G34a*(2), I.7**, -L10, -L13, L15, L15a, L15b, -L18, -L19, 163.

Mammalia: G2[14-28], G3[14-28], G4[14-28], G4a[14-28], G5a[14-28], G5b[14-28], G6[14-28], G7(2)[14-28], G8(2)[14-28], G10[14-28], G11b[14-28], G12[14-28], G14[14-28], G15[14-28], G20[14-28], G20a[14-28], G21**[14-28], G34b**[1, 14-28], G34c*[14-28], G36a(2), G37(2)**[1, 14-28], L4[14-28], L7(3)[14-28], I.9[14-28], A7[14-28], A12[14-28], A13[14-28], A21[14-28], A26[14-28], A32, $70(2),-135,154[28]$.

Morganucodontidae: none.

Ophiacodon: $15^{* *}, 131,-141^{* *}$.

Ornithosuchia: (most of these characters are observed in birds only, and their origins within Ornithosuchia are unknown) G2, G4, G4a, G5a, G5b, G6, G7(2)*, G8(2), G10, G12, G13(2)[5-12], G14, G15, G20a, L4, A13, - A14*, A26, - 127, - 128, $-129 * *$ (Euparkeria only).

Procynosuchus: none.

Proterochampsidae: $9,58,129 * *$.

Proterosuchidae: none.

Protorosauria: none.

Pseudosuchia: (the level of generality of these characters may not be clear, but they are present in crocodilians) $\mathrm{G} 7 *,-\mathrm{Al1}, 129 * *$.

Rhynchosauria: 30.

Sphenacodontinae: none.

Testudines: (soft characters diagnose Chelonia, but they may have originated earlier in 
Testudines) G13, -G24a*, G34**[13], G34c**[13], 9, 16, 48, 50, 68, 127, 128, $139 * *,-148 *, 160,164,187,188,189,190,192,207$.

Therocephalia: $57(2), 58,-123 * *,-182 * *$.

Thrinaxodon: $125 * *$.

Trilophosaurus: $16^{* *},-37,70(2), 115,117,120,121,123$.

Tritylodontidae: $30^{* *}, 111,-125^{* *}$.

\section{Appendix B}

Most of the following diagnoses of the major groups of Recent Amniota, namely, Reptilia, Sauria and Archosauria, result from the present research. The reader is referred to Gaffney (1975) and Gauthier et al. (1988a) for diagnoses of Chelonia and Lepidosauria, respectively. Our descriptions record all synapomorphies discovered in the parsimony analysis (Fig. 3) of the data in Tables 1 (soft anatomy only) and 2. The lists constitute diagnoses of groups delimited by extant taxa only, and thus they may include characters that are known (hard anatomy) or suspected (soft anatomy) to have arisen earlier than indicated. Nevertheless, characters listed as diagnostic for each taxon were certainly absent in the common ancestor that group shared with other extant amniotes. As in $\Lambda$ ppendix $A$, ambiguous characters highlighted with an asterisk have been added to call attention to the need for further research. In some instances we have chosen a particular interpretation, according to either Gauthier (1984) or Rowe (1986a), or based on our discussions of optimization immediately following character descriptions in the text. The characters are presented in the order in which they are listed in $\Lambda$ ppendix $\Lambda$. We have also added Rowe's (1988) diagnosis of Mammalia. The hard characters emphasize the distinctness of Mammalia from all other synapsids, including Morganucodontidae. While the soft characters further delimit Mammalia from all extant amniotes, they may have greater generality among the extinct synapsids. Almost all of the synapomorphies in Appendix $A$ which appear at nodes 14-28, plus those listed for Mammalia, provide additional diagnostic information, and they might have been listed as well.

Reptilia: phi keratins; pyramidalis muscle*; nictitans tendon attaches to pyramidalis muscle*; large and highly mobile nictitans*; cricoid cartilage consists of two or more tracheal rings; ornithuric acid produced in benzoic acid metabolism; external nasal gland lies outside nasal capsule; color vision; dorsoventricular ridge of telencephalon; iris composed of striated muscle; masticatory muscle plate divides into $m$. constrictor dorsalis and $m$. adductor mandibulae; fewer than three postmandibular branchial arches; supratemporal small and separated from postorbital; tabular-opisthotic contact absent*; horizontal ventral margin of postorbital region of skull; large post-temporal fenestra; tabular reduced; suborbital fenestra; supraoccipital narrow, with anterior crista; anterior coronoids absent; ectopterygoid teeth absent*; second intercentrum fused to first centrum in adult; cervical centra keeled ventrally**; medial centrale in pes absent.

Sauria: septum sinu-venosi*; tendon to lower eyelid absent*; specialized adrenal gland*; low urea concentration in blood plasma; olfactory peduncles link olfactory bulbs to brain; kidney closely appressed to adrenal gland; Huxley's foramen; postorbital region of skull shorter than preorbital; temporal musculature originates dorsally on parietal table; lacrimal excluded from external naris by nasal-maxilla contact; upper temporal fenestra present and dorsolaterally oriented; lower temporal 
fenestra present and laterally oriented; suborbital fenestra large; quadrate exposed laterally; exoccipitals not in contact on occipital condyle; basi-occipital forms most of occipital condyle; small and posteriorly directed retroarticular process; caniniform maxillary teeth absent; second intercentrum broadly participates in craniovertebral joint; sacral ribs directed laterally; supraglenoid buttress absent; posterior coracoid absent; clavicles narrow medially; interclavicle $\mathrm{T}$-shaped; long slender limbs; dorsal groove on ilium absent; posterior process of ilium reaches posterior end of ischium; ilium expanded anteriorly and dorsally, with horizontal dorsal margin; ilium occupies $80-85 \%$ of acetabulum; acetabulum completely ringed by ridge of bone (cotyloid notch absent); acetabulum rounded; femoral intertrochanteric fossa reduced; femoral condyles subequal; femoral ventral ridge system feebly developed; ridge and groove tibioastragalar joint; proximal carpals and tarsals relatively small; long slender hands and feet; fifth metatarsal short and broad.

Archosauria: pulmonary artery with three semilunar valves; heart with muscular lateral valve to right of auriculo-ventricular orifice; some enlargement and infolding of lateral hemispheres of cerebellum (and associated modification of pons varolii, and inferior and pontine nuclei); postconcha in antorbital space of cavum nasi proprium; pancreas nestled in first duodenal loop of small intestine, binding parts of loop together; heart with complete interventricular septum; posthepatic septum; renal portal system bypass via renal vein and subcardinal; thickened and convoluted Reissner's membrane in inner ear; eustachian tube passes through cranial base via single opening in roof of pharynx; transparent nictitans; subclavians originate anterior to separation of internal and external carotids; dorsoventral growth of cell cords of pars distalis of pituitary gland; vomeronasal organ absent beyond early embryo; specialized adrenal gland histology*; novel thymus morphology; three neurofilament proteins (new high molecular weight polypeptide); most of lumen of stomach lies posterior to pyloris; gizzard (prominent lunica muscularis); urinary bladder absent; nest built out of vegetation and some parental care of hatchlings; cell clusters evenly distributed in anterior dorsoventricular ridge of telencephalon; well-developed cochlear, angular and magnocellular nuclei in rhombencephalon; part of puboischiofemoralis muscle has dorsal origin; premaxilla(e) large, forming most of tip of snout; premaxilla with large postnarial process, excluding maxilla from naris; nasal longer than frontal; prefrontal small; parietal foramen absent; postparietals fused; postparietal absent in adult; supratemporal absent; tabular absent in adult; antorbital fenestra; antorbital fossa; jugal extends to posterior end of skull; upper temporal fenestra small and dorsally oriented; post-temporal fenestra small; pyriform recess extends to anterior ends of palatines; squamosal broadly separated from ventral margin of skull; otic notch present; L-shaped quadratojugal; stapes rod-like; stapedial foramen absent; craniomandibular joint posterior to occiput; laterosphenoid ossification in braincase; paroccipital process expanded distally; paroccipital process directed posteriorly; exoccipital fused to opisthotic in adult; mandibular fenestra; teeth in deep sockets; teeth attached by periodontal ligament; teeth serrated; lower tooth row terminates anterior to upper; premaxillary teeth in deep sockets; caniniform anterior dentary tooth; teeth absent on transverse process of pterygoid; notochord absent in adults; apex of neural spines expanded at base of neck; presacral intercentra absent (except first and second); cervical ribs three-headed and rib shafts parallel cervical centra; parasagittal 
osteoderm rows; scapula narrow and long; cleithrum absent; interclavicle arms short; humeral epicondyles reduced; ectepicondylar groove in humerus absent; entepicondylar foramen in humerus absent; third digit longest in manus and pes; inner two digits stouter than outer two in manus and pes; intertrochanteric fossa of femur absent; femoral head inflected; distal femoral condyles not expanded broadly beyond shaft; femoral shaft prominently sigmoidal; raised fourth trochanter; curved tibio-astragalar joint; calcaneal tuber directed posteriorly; centrale fused to astragalus in adult; first distal tarsal absent; fifth distal tarsal absent; fifth metatarsal hooked.

Mammalia: single aortic trunk; pulmonary artery with three semilunar valves; endothermy; incubation of eggs; erector muscles and dermal papillae; three meninges; folded cerebellum, pons varolii, inferior olive and pontine nuclei; adventitious cartilage; vascularized islets of pancreas; renal macula densa; loop of Henle; completely divided heart with thick, compact myocardium; loss of the sinus venosus and development of a sinu-venosi septum**; absence of tendon to lower eyelid**; intrinsic eyelid muscles derived from facial platysma; subclavian arteries positioned anteriorly, near the carotids**; auriculo-ventricular node and Purkinje fibers; unique adrenal histology; three neurofilament polypeptides; most of stomach lumen lies posterior to pyloris; nest building; ependymal cells fail to reach periphery of brain in adults; processus recessus encloses anteriormost remnant of fissura metotica; calcite otoliths; $m$. panniculus carnosus forms continuous sheath of muscle wrapping trunk and neck; muscular diaphragm encloses pleural cavities, and consequent development of diaphragmatic breathing; superficial musculature expanded onto face and differentiated into muscle groups associated with eye, ear and snout; elaborate development of greater omental bursa; epiglottis; welldeveloped hippocampus; dorsal or hippocampal commissure and anterior commissure interconnect pallial structures of two cerebral hemispheres; motor nucleus of facial nerve expanded and divided into nucleus facialis dorsalis and nucleus facialis ventralis; strong representation of facial nerve field in motor cortex; restriction of sensory field of facial nerve and great expansion of cutaneous field of trigeminal nerve over face; chorda tympani passes below stapes; divided optic lobes; well-developed specific motor nuclei which receive afferents from cerebellum or basal ganglia, project to restricted regions of telencephalon, and are situated rostrally in ventral half of thalamus; central region of telencephalic pallium is isocortex; hindbrain overlies fenestrae vestibuli; thrombocytes take form of blood platelets; erythrocytes lack nuclei; adult liver and spleen play only minor role in erythrocyte formation; hair; sebaceous glands; sweat glands; mammary glands; parotid, submaxillary and sublingual glands; tympanic membrane with middle layer or membrana propia; thymus differentiates from ventral part of gill pouch of second postspiracular gill cleft; cervical thymus gland forms from invagination of ectoderm of neck; lungs expanded ventrally, surrounding heart and almost meeting on ventral midline, leaving only median strand of tissue, the ventral mediastinum, connecting pericardial sac with ventral body wall; complex lung structure with division of lungs into lobes, bronchioles and alveoli; adults without prenasal process of premaxilla, rendering external nares in skeleton confluent; sclerotic ossicles absent; tabular absent; adult craniomandibular joint formed exclusively by squamosal and dentary; adult craniomandibular joint positioned entirely anterior to fenestra vestibuli; adult middle ear comprised of at least six separate ossifications, 
incus (quadrate), malleus (articular), ectotympanic (angular), os goniale (prearticular), ossiculum accessorium mallei (surangular), and stapes that are suspended from skull; absence of notches in squamosal for quadrate and quadratojugal; quadratojugal not a separate ossification; ossified ethmoid turbinals and internasal septum; ossified cribriform plate; lateral pterygoid flange vestigial and widely separated from mandible; inflated, pneumatic mastoid process and down-turned paroccipital process; tegamen tympani forms de novo cranial wall; stylohyal fused to auditory capsule to form styloid process; stapes very small and imperforate; occipital condyles expanded dorsally to enclose ventral two-thirds of foramen magnum, and traverse wide arc of abduction; Meckelian sulcus enclosed by dentary to form Meckelian canal; coronoid bone vestigial and fused to medial surface of dentary; proatlas absent postembryonically; atlas intercentrum and neural arches fused to form single, ring-shaped osseous structure; atlantal rib absent; axial prezygapophysis absent; postaxial cervical ribs fused to their centra, forming transverse foramina; epiphyses on long bones and girdles; patella and patellar facet on femur; presence of parafibular flabellum; flexor sesamoids in manus and pes; styloid process of radius, tibia and fibula; saddle-shaped articulation between entocuneiform and first metatarsal. 